\title{
Large-Scale Cluster-State Entanglement in the Quantum Optical Frequency Comb
}

\author{
Moran Chen \\ Nanchong, Sichuan, China \\ B.S. Physics, Beijing Normal University, 2009 \\ A Dissertation presented to the Graduate Faculty \\ of the University of Virginia in Candidacy for the degree of \\ Doctor of Philosophy \\ Department of Physics \\ University of Virginia \\ May 2015
}


(c) Copyright

All Rights Reserved

Moran Chen

Ph.D., May 2015 


\section{Acknowledgments}

First of all, I would like to thank my adviser, Prof. Olivier Pfister. Without his help and guidance, all of the research and work described in this thesis would have been impossible. I am wholeheartedly grateful to have him as my adviser. He gave me enough freedom and trust to allow me to conduct research and independent thinking on my own, and his incredible patience and knowledge in both the theory and in the experiments also gave me confidence and reassurance when I came across obstacles and difficulties. He is also always encouraging and kind, and is the best adviser I could have hoped for. He has led me into the amazing field of quantum optics, and I am truly blessed to have him as my adviser.

I want to give special thanks to our theoretical collaborator Nick Menicucci. Nick is an outstanding theorist and a very smart, quick and straightforward person, and it has always been a pleasure talking with him about research topics or other general issues. We had many good and interesting discussions and his genius ideas and simplified theoretical proposals helped make our experiment easier and more feasible. I am truly thankful to have him as our theoretical collaborator and friend.

Also, I would like to thank my lab mates and colleagues for their help, support and friendship, which made the cold lab an enjoyable place at which to work. I thank Matt Pysher for introducing and teaching me the experimental procedures and skills, Reihaneh for her support and especially for her strong and warm friendship, 
Niranjan for his discussions and many fun and thought-provoking conversations, Pei and Wenjiang for their support and help. All of them helped make my research and Ph.D. experience easier, more pleasant and a whole lot more fun. It has been a great pleasure working and spending time with them.

For my family I always cannot thank them enough for their unconditional love and support. My parents are always very giving and supportive; especially my mom, who has always been my best and most understanding friend, listening to me, feeling for me and supporting me through all of the good and bad times. My grandparents, to whom I owe so much, have always been proud of me and caring for me, even though they have no idea what my research is about. I also thank my husband Jason for his deep love and unconditional support, especially through difficult times, which makes my Ph.D. experience and life easier, more fun and complete.

I also want to thank all of my friends for the friendship they give me, the happy moments and cheerful times together in our beautiful town of Charlottesville. They make my $\mathrm{Ph} . \mathrm{D}$. life more enjoyable and their friendship is invaluable. 


\begin{abstract}
Ever since its discovery, quantum mechanics has remained an intensely active field, and its real-world applications continue to unfold rapidly. In 1982, Richard Feynman proposed a new type of computer operating directly under quantum mechanics laws: the quantum computer [1]. Compared with the classical computer, whose information is encoded in bits, the quantum computer, whose information is encoded in quantum bits, or qubits, will be able to perform calculations exponentially faster for such problems as factoring large integers into primes and simulating complicated quantum systems. Due to their extremely powerful calculation speeds and abilities, quantum computers have been the long-pursued dreams for both experimentalists and theorists in many research groups, government agencies, industrial companies, etc., and the fast-paced developments in their architecture and speed continue to make them more and more attractive. There are two principal models of quantum computing: the circuit model and the measurement-based model. The circuit model is similar to a traditional computer where there are inputs, gates and outputs. The measurementbased model is different, as it is crucially based on the cluster state, a type of highly entangled quantum state. In this new model, quantum computing begins with an initial cluster state and then carries out calculations by physical measurements of the cluster state itself along with feedforward. Thus, the cluster state serves as the material and resource for the entire set of calculations, and it is an extremely important part of measurement-based quantum computing.
\end{abstract}


This thesis will discuss an experimental and theoretical work that holds the world record for the largest entangled cluster state ever created whose 60 qumodes (optical versions of qubits) are all available simultaneously. Moreover, the entangled state we created is not random, and it is a cluster state which meets the specific requirements for implementing quantum computing. In the race to build a practical quantum computer, the ability to create such a large cluster state is paramount. Also, our creative optical method to generate massive entanglement advances many other methods due to its high efficiency, super-compactness and large scalability. The entanglement proceeds from interfering multiple EPR entangled pairs, which are generated from the down-converting process of a nonlinear crystal in an optical parametric oscillator, into a very long dual-rail wire cluster state. Moreover, many copies of the same state can easily be obtained by merely adjusting the frequencies of the pump lasers. These cluster states serve as building blocks of the universal quantum computer, and also are, in their own right, important resources for studying and exploring quantum mechanics in large systems. 


\section{Contents}

Acknowledgments . . . . . . . . . . . . . . . . i i

Abstract .......................... ii

1 Introduction 1

2 Quantum Optics 8

2.1 Introduction . . . . . . . . . . . . . . . . . 8

2.2 Basic concepts. . . . . . . . . . . . . . . . 9

2.2.1 The harmonic oscillator . . . . . . . . . . . . . 9

2.2.2 Three evolution pictures . . . . . . . . . . . . . . 11

2.3 The beam splitter . . . . . . . . . . . . . . . . . . 19

2.4 Quantum states ........................ 22

2.4.1 Fock state . . . . . . . . . . . . . . . . . 22

2.4.2 Coherent state . . . . . . . . . . . . . 26

2.4.3 Squeezed state . . . . . . . . . . . . . . . . 28

2.5 Squeezing generation . . . . . . . . . . . . . . . . 33

2.5.1 Single-mode squeezing . . . . . . . . . . . . 33

2.5.2 Two-mode squeezing . . . . . . . . . . . . . . . 36

3 One-way Quantum Computing $\quad 42$

3.1 Introduction . . . . . . . . . . . . . . . . . . . . . . . . . . . . . . . .

3.2 Cluster states . . . . . . . . . . . . . . . . . . . 44

3.2.1 Generation and definition . . . . . . . . . . . . 44

3.2.2 Properties . . . . . . . . . . . . . . . 47

3.2.3 Comparison with other entangled states . . . . . . . . . . 49

3.3 One-way computing using cluster states . . . . . . . . . . . . 52

3.3.1 Quantum teleportation in a wire............ 53

3.3.2 An arbitrary rotation $U_{R} \ldots \ldots \ldots \ldots$. . . . . . . . 55 
3.3.3 Two-bit operation: CNOT gate . . . . . . . . . . . 56

3.3 .4 Quantum circuits . . . . . . . . . . . . 57

3.4 From qubits to qumodes:

the continuous-variable cluster state . . . . . . . . . . . . 60

3.4.1 Qubit to qumode generalization . . . . . . . . . . . 60

3.4.2 Gaussian and non-Gaussian operations . . . . . . . . . . 65

3.4.3 Continuous-variable cluster state definition . . . . . . . . . 67

4 The Optical Parametric Oscillator $\quad 70$

4.1 Constituents . . . . . . . . . . . . . . . . . . . . . . 71

4.2 Optical cavity . . . . . . . . . . . . . . . . . . . . 72

4.2 .1 Free spectral range . . . . . . . . . . . . . . . . 73

4.2 .2 Linewidth and Finesse . . . . . . . . . . . . . . 75

4.2 .3 Intracavity loss $\ldots \ldots \ldots \ldots$

4.3 Nonlinear optics . . . . . . . . . . . . . . . . . . . . . . . . . . . . . 89

4.3.1 Second-order nonlinear optics _ . . . . . . . . . . . . 90

4.3.2 Quasi phase matching . . . . . . . . . . . . . . 93

5 60-mode Multipartite Entanglement $\quad 96$

5.1 Previous proposals overview $\ldots \ldots \ldots \ldots$

5.2 Principles of experiments . . . . . . . . . . . . . . . . . . . . 101

5.2 .1 Qualitative analysis . . . . . . . . . . . . . . . . 101

5.2 .2 Theoretical analysis . . . . . . . . . . . . . . . 106

5.2.3 Analysis of unequal squeezing parameters . . . . . . . . . . 110

5.2.4 Generation to multiple wires . . . . . . . . . . . . . . 111

5.3 Experimental setup and equipment . . . . . . . . . . . . . 113

5.3 .1 Experimental setup . . . . . . . . . . . . . . . . . . . . 113

5.3 .2 Experimental equipment . . . . . . . . . . . . . . . . 115

5.3 .3 Basic experimental methods . . . . . . . . . . . . . . 117

5.3 .4 Backward reflection . . . . . . . . . . . . . . . . . 132

5.4 Experimental results . . . . . . . . . . . . . . . . . . . . . 145

5.4 .1 One-wire case . . . . . . . . . . . . . . . . . . . . . . . 145

5.4 .2 Two-wire case . . . . . . . . . . . . . . . . . . . . . 152

5.4 .3 Electronic noise correction . . . . . . . . . . . . . . 157

5.5 The van Loock-Furusawa inseparability criteria . . . . . . . . . 159

5.5.1 One mode- three mode bipartitions . . . . . . . . . . . 161 
5.5.2 Two-mode bipartitions . . . . . . . . . . . . . . . . . . 162

5.5.3 Sufficient conditions for inseparability . . . . . . . . . . . 162

6 Conclusion

$\begin{array}{ll}\text { A Appendix } & 167\end{array}$

A.1 Pump spectra manipulation . . . . . . . . . . . . . . . . . 167

A.1.1 Single-sideband Modulation . . . . . . . . . . . . . . 168 


\section{List of Figures}

2.1 Beam Splitter . . . . . . . . . . . . . . . . . . . . . . . 19

2.2 Phasor diagram for the Fock state . . . . . . . . . . . . 25

2.3 Phasor diagram for the coherent state . . . . . . . . . . . . . 28

2.4 Phasor diagram for the squeezed vacuum state . . . . . . . . . . . . 32

3.1 Example of Cluster state $C \ldots \ldots \ldots$. . . . . . . . . 48

3.2 Example of quantum computation in a cluster state lattice . . . . . 53

3.3 Cluster structure for CNOT gate . . . . . . . . . . 56

3.4 Graph A for EPR state . . . . . . . . . . . . . . . . . 69

4.1 Typical OPO building parts . . . . . . . . . . . . . . 71

4.2 OPOs with different optical cavity forms . . . . . . . . . . . 72

4.3 Optical Frequency Comb of an $\mathrm{OPO} \ldots \ldots . \ldots 74$

4.4 The reflection and transmission of an optical cavity . . . . . . . 75

4.5 Plot of OPO transmittance $T$ as a function of round trip optical phase $\delta 78$

4.6 OPO transmittance $T$ as a function of round trip optical phase $\delta$ for two different Finesses associated with two different $R$ s . . . . . . . . . 80 
4.7 Comparison of the original finesse definition with the approximated

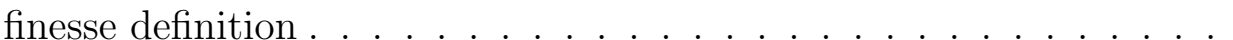

4.8 Comparison of the one-sided cavity finesse with the two-sided cavity

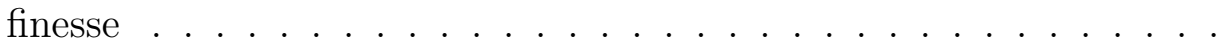

4.9 The setting to measure the OPO intracavity loss . . . . . . . . . . 84

4.10 A typical reflection signal for intracavity loss measurement . . . . . . 85

4.11 The intracavity loss $L$ versus $k$ for a one-sided OPO with output cou-

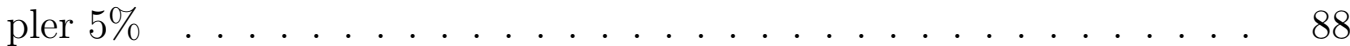

4.12 The effect of the phase mismatch $\Delta k L \ldots \ldots \ldots$. . . . . . . . . . 94

5.1 Crown quantum entangled state . . . . . . . . . . . . . . . . . . 99

5.2 An OPO with a $z z z$ nonlinear crystal . . . . . . . . . . . . . 101

5.3 Quantum Optical Frequency Comb (QOFC) of the OPO . . . . . 102

5.4 Reorganizing the $z z z$ EPR pairs to order them in a sequence . . . . . 102

5.5 The OPO with two orthogonal nonlinear crystals . . . . . . . . 103

5.6 The OFC for two polarizations . . . . . . . . . . . . . . . . 104

5.7 Reorganize the yyy EPR pairs to align them in an array . . . . . . . 104

5.8 The separate $z z z$ and $y y y$ EPR pairs connect into a dual-rail structured wire after the beam splitter . . . . . . . . . . . . . . 105

5.9 Generation of a dual-rail quantum wire in the QOFC (with pump index and weights labeled) . . . . . . . . . . . . . 106

5.10 Generation of two dual-rail quantum wires . . . . . . . . . . . . . . 112 
5.11 Experimental setup for the 60-mode cluster state . . . . . . . . . . 114

5.12 Typical experimental arrangement for FM spectroscopy . . . . . . . . 118

5.13 Different error signals after demodulation for different modulation fre-

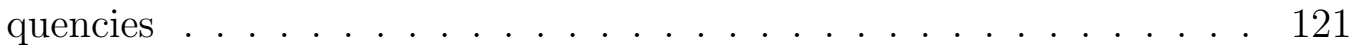

5.14 Typical PDH servo loop to lock the frequency of a laser to a FabryPerot cavity ........................... 122

5.15 Typical phase lock loop between two lasers . . . . . . . . . . . . . 123

5.16 Phase lock loop between two lasers with AOM to lock at the same frequency . . . . . . . . . . . . . . . . . 125

5.17 The balanced homodyne detector . . . . . . . . . . . . . . 126

5.18 Visibility figures . . . . . . . . . . . . . . . . 131

5.19 The experimental setup for measuring the back-reflection power of our

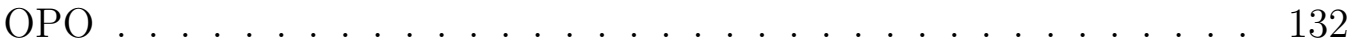

5.20 The back-reflection power's dependence on the input power . . . . . . 134

5.21 The back-reflection power's dependence on the temperature . . . . . . 135

5.22 Simultaneous measurements for intracavity loss and the back-reflection 136

5.23 The reflection and transmission paths when a light wave hits a crystal 137

5.24 The reflectivity and transitivity of a crystal as index of refraction $n$ changes . . . . . . . . . . . . . . . . . . 138

5.25 The "forward propagating" mode and the "backward propagating" mode coupled by the cavity formed by the PPKTP crystal . . . . . . 140 
5.26 The model for the system where the PPKTP crystal serves as the coupling cavity between the forward and backward prorogating modes

5.27 Coupling cavity model simulation . . . . . . . . . . . . . . . . 143

5.28 Visualization of the experimentally measured nullifiers on the dual-rail graph state structure . . . . . . . . . . . . . . . . . 145

5.29 Zero-span spectrum analyzer traces of raw squeezing measurements for $m=1$ quantum wire . . . . . . . . . . . . . . . . 146

5.30 "Wrong" modes checks for one-wire case . . . . . . . . . . . . . . . . 147

5.31 The squeezing measurements for the y pump centered nullifiers in the

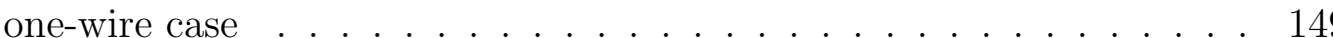

5.32 The squeezing measurements for the $\mathrm{z}$ pump centered nullifiers in the one-wire case . . . . . . . . . . . . . . . . . . . . 150

5.33 Wrong-frequency measurements for one-wire case . . . . . . . . . . 151

5.34 Zero-span spectrum analyzer traces of raw squeezing measurements for $m=2$ quantum wire . . . . . . . . . . . . . . . . 152

5.35 "Wrong" modes checks for two-wire case . . . . . . . . . . . . . . . . 153

5.36 The squeezing measurements for the y pump centered nullifiers in the

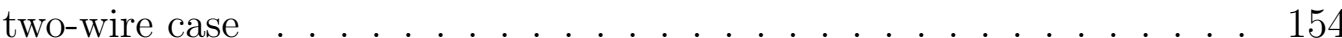

5.37 The squeezing measurements for the $\mathrm{z}$ pump centered nullifiers in the two-wire case . . . . . . . . . . . . . . . . . . 155

5.38 Wrong-frequency measurements for two-wire case . . . . . . . . . 156 
5.39 Electronic noise measurement . . . . . . . . . . . . . . . . 157

A.1 The 15-frequency pump spectrum . . . . . . . . . . . . 167

A.2 The 3-frequency pump spectrum . . . . . . . . . . . . . . . . . 168

A.3 The setup to generate single sideband . . . . . . . . . . . . . . . . . . 169

A.4 The frequency spectra of corresponding points in Figure A.3 . . . . . 170

A.5 Bessel functions of different orders . . . . . . . . . . . . . . . . 171 


\section{Chapter 1}

\section{Introduction}

Quantum computing, simply stated, is an effort to implement computing tasks or information processing using quantum mechanical laws.

The quantum computer was first proposed by Richard Feynman in 1982. He pointed out the possibility of building a computer following the laws of quantum mechanics directly to simulate quantum systems because the difficulty of simulating a quantum system on a classical computer grows exponentially with the size of the quantum system [1]. Also, trying to derive a stronger version of the Church-Turing thesis, Deutsch asked whether a computer built on quantum mechanics rules can exceed the Turing machine, even the probabilistic Turing machine, and he then constructed a computational example that indeed shows the advantage of the quantum computer over the classical computer [2,3]. In 1994, Peter Shor demonstrated two important problems (finding the prime factors for an integer and the "discrete logarithm" problem) could be solved much faster on a quantum computer than on a classical computer [4]. Since then the field of quantum computing has been receiving 
research attention and been developing rapidly.

On the classical computing end, the invention of the transistor and other techniques have enabled its growth and development in an amazing pace [5]. Gordon Moore, in 1965, made an observation-based statement, now known as the Moore's law, that the computer power will double for constant cost roughly once every 18 months [6]. Amazingly, the development of the classical computer has thus far been approximately following Moore's law; however, as the computer hardware becomes smaller, faster and more compact, the constitution of chips is getting closer to enumerable atoms, hence to the border of the quantum realm.

We could include the quantum algorithms to conduct the computing, following the quantum mechanics rules, as a way to enhance, if not substitute for, the classical algorithms.

As previously mentioned, quantum computing has the ability to carry out some complicated computation tasks more efficiently than classical computing. It is believed that it is impossible for classical computer to efficiently perform some calculation and simulation tasks (such as prime factorization of integers or simulation of complicated systems), while it is believed that quantum computers will not have this problem.

Efficient is an important concept in time complexity (the amount of time taken by an algorithm to run as a function of the length of the input) in computer science (also referred to as quick, tractable, fast, etc.). Here, by "efficient", we mean that 
the time $T(n)$ needed to solve a problem is a polynomial of the size $n$ of the problem, $O\left(n^{k}\right)$, where $k$ is a constant, and such problem lies in the complexity class $P[7,8]$. In contrast, "inefficient" means that the time required is super-polynomial $(T(n)=$ $\omega\left(n^{k}\right)$, i.e., $T(n) \geq c n^{k}$ where $c, k$ are constants), often exponential $\left(k^{n}\right)$.

Classical computation uses the classical bit as the information carrying unit; a bit can have values either 0 or 1 , but not both. Quantum computation, on the other hand, uses quantum bit, or qubit, as the computing unit, and due to the superposition principle of quantum mechanics, a qubit can have states of $|0\rangle,|1\rangle$, or a linear combination of both:

$$
|\psi\rangle=\alpha|0\rangle+\beta|1\rangle
$$

When we have $n$ qubits, there will be $2^{n}$ basis states. Compared with only one state for the classical case, this quantum parallelism provides huge room for the entanglement, thus provides great potential for the quantum computer to outperform classical computer, given that appropriate algorithms are found for particular problems [9].

There are two major challenges towards building a practical quantum computer with good computing capability: overcoming decoherence and realizing scalability [10]. Decoherence can be overcome by adopting more robust quantum systems and using efficient error correction algorithms $[9,11,12]$. Traditional ways to achieve scalability are to repeatedly build and add on the quantum memory unit - the "bottom up" 
method. Another approach is to generate all of the quantum units at once through some well-designed mechanism - the "top down" method. In my thesis, I will talk about our "top down" method to achieve large scalability for quantum computing.

In terms of the quantum memory unit, we have two flavors, the first being the discrete variable, or qubit, and the second being the continuous variable, ubiquitous amplitudes of a quantum harmonic oscillator, or qumode. Traditionally the information is encoded on the qubit [13-28], but the continuous variable qumode, especially optical qumodes, offers great advantages such as experimental convenience, less interaction with the environment and great potential for scalability [29-37]. Very recently, the discovery of threshold for the fault-tolerance one-way continuous variable QC further strengthened the continuous variable case [11].

The one-way quantum computer, proposed by Raussendorf and Briegel [38], is a type of measurement-based quantum computer. Compared with the circuit-based quantum computer, where the qubit inputs are going through a sequence of gates to carry out the calculation, the one-way quantum computer does all of the calculations by implementing measurements on a highly entangled cluster state made beforehand. The cluster state is thus a crucial part of one-way quantum computing because it provides the resource and material for the whole calculation, and it is where everything begins. Eventually the results of the calculations will be embedded on the last qubit(s) to be extract (or qumode(s) if it is continuous variable QC), after all of the wellselected and arranged measurements. Chapter 3 has more details about one-way 
QC.

Many effects and different degrees of success have occurred during the creation of a cluster state and demonstration of one-way quantum computing [13,35, 37, 39-43]. In 2005 a four-qubit cluster state was realized using the polarization state of four photons, and the feasibility of one-way quantum computing was demonstrated [13]. In 2008 a four-mode continuous variable cluster state was experimentally generated [41]. In 2011,15 copies of four-mode continuous variable cluster states were generated simultaneously over a single optical parametric oscillator [35]. In 2013, a 10,000 mode cluster state was realized over the time domain, with a few modes available at a given time [37]. Recently the first experimental realization of one-way quantum computer algorithm solving Simon's problem using five-qubit cluster state was achieved [42].

In this thesis, I am going to discuss the work I have done in terms of building a largely scalable continuous variable cluster state. The cluster state has a dual-rail wire structure, and the confirmed length of this "long wire" is 60 qumodes, limited only by the measurement technique [34]. (An estimate according to the phase matching curve of the nonlinear crystal used in the experiment indicates thousands of modes are actually generated [44].) Furthermore, this cluster state that we generated can be used to build higher-dimensional cluster states (square-grid, lattice, hypercube, etc.), which has great potential applications for quantum computing and quantum information [45].

This 60-mode cluster state set the world record for the largest entangled cluster 
state ever created in which all of the constituent quantum systems are individually addressable and simultaneously available. The largest entangled state of any sort is 10, 000 modes [37], but its constituents do not exist simultaneously - their modes are emitted in pairs and detected in turn. The modes come in time packets and are not all available, with only a small portion of the states existing at a given time. It can be used for some measurement-based quantum computation based on sequential applications of quantum teleportation, but our state is distinguished from it in the sense that all of our modes have simultaneous availability and our state exists continuously. However, these two states use similar construction concepts proposed by Nicholas Menicucci [46]: our state is in the frequency domain, while their state is in the time domain, and both to some extent compliment each other and showed beautifully that the same idea works in both domains.

Details of generating our 60-mode cluster state can be found in Chapter 5.

This thesis has 6 chapters and an appendix:

This chapter (Chapter 1) gives a brief introduction to quantum computing, oneway quantum computing, and the cluster state. It also briefly introduces the 60 -mode dual-rail wire cluster state we built and highlights its significance. Chapter 2 is an introduction to the subject quantum optics. It covers basic concepts and definitions and introduces typical quantum states such as the squeezed states which we use in our experiments. Chapter 3 summarizes the definition and characteristics of oneway quantum computing, a measurement-based quantum computing and its essential 
element cluster states, as well as the corresponding relations and transitions between the discrete variable qubit and its continuous variable counterpart qumodes. Chapter 4 is dedicated to our core experimental tool, the optical parametric oscillator (OPO). The OPO's basic constituents and various important concepts and definitions are also discussed. Chapter 5 is the main chapter, describing the generations of the 60mode large cluster state. I start from our experimental principles and the current method's differences and advantages over the previously proposed methods. Then, I move on to introduce our experimental methods and setups and briefly cover some important basic experimental techniques. Meanwhile, some experimental problems we solved are discussed. Finally, the experimental results are shown together with their explanations and interpretations, and the proofs of the inseparability of our cluster state are shown. The last chapter, Chapter 6 , is a conclusion of the thesis and the experimental results. Some previously proposed methods and revelent experiments can be found in the Appendix. 


\section{Chapter 2}

\section{Quantum Optics}

\section{$2.1 \quad$ Introduction}

Quantum optics is different and newer than classical optics. As the name suggests, quantum mechanics knowledge is used to explain the optical phenomena. It studies the light's particle, as well as wave, property, by considering light particles, i.e., photons. This treatment leads to different results from the "intuitive" classical optical pictures for some experiments. In the following sections I will first review briefly quantum mechanics background and then move on to quantum optics (note this is far from a complete treatise on quantum optics, just a few topics that are most relevant to my research). The references for this chapter are my adviser Prof. Olivier Pfister's lecture notes [47] and Mark Fox's Quantum Optics - an introduction [48]. 


\subsection{Basic concepts}

\subsubsection{The harmonic oscillator}

The hamiltonian for a one-dimensional harmonic oscillator is

$$
\begin{aligned}
H & =\frac{p^{2}}{2 m}+\frac{1}{2} k x^{2} \\
& =\frac{p^{2}}{2 m}+\frac{1}{2} m \omega^{2} x^{2},
\end{aligned}
$$

where the resonant frequency of the oscillator defined as $\omega=\sqrt{\frac{k}{m}}$ was used, and $p$ is the momentum and $m$ is the mass.

The time-independent Schrödinger equation is:

$$
H \psi(x)=-\frac{\hbar^{2}}{2 m} \frac{d^{2} \psi(x)}{d x^{2}}+\frac{1}{2} m \omega^{2} x^{2} \psi(x)=E \psi(x) .
$$

The solutions are:

$$
\psi_{n}(x)=u_{n}(x) e^{-m \omega x^{2} / 2 \hbar}
$$

with quantized energy levels:

$$
E_{n}=\left(n+\frac{1}{2}\right) \hbar \omega
$$

where $u_{n}(x)$ is related to the Hermite polynomials and $\mathrm{n}$ is an integer $n \geq 0$.

Now define

$$
\begin{aligned}
& P=\sqrt{\frac{1}{m \omega \hbar}} p \\
& Q=\sqrt{\frac{m \omega}{\hbar}} x
\end{aligned}
$$


The annihilation and creation operators $a$ and $a^{\dagger}$ are defined respectively as:

$$
\begin{aligned}
& a=\frac{1}{\sqrt{2}}(Q+i P) \\
& a^{\dagger}=\frac{1}{\sqrt{2}}(Q-i P)
\end{aligned}
$$

The hamiltonian becomes:

$$
\begin{aligned}
H & =\frac{\hbar \omega}{2}\left(P^{2}+Q^{2}\right) \\
& =\hbar \omega\left(a^{\dagger} a+\frac{1}{2}\right) \\
& =\hbar \omega\left(N+\frac{1}{2}\right)
\end{aligned}
$$

where $N$ is the photon number operator $N=a^{\dagger} a$.

This is consistent with the classical harmonic's energy levels Eq.(2.4).

The commutation relation of the annihilation and creation operators is:

$$
\left[a, a^{\dagger}\right]=1
$$

Thus, the operators $Q$ and $P^{\prime}$ 's commutation relation is

$$
\begin{aligned}
{[Q, P] } & =\left[\frac{1}{\sqrt{2}}\left(a+a^{\dagger}\right), \frac{-i}{\sqrt{2}}\left(a-a^{\dagger}\right)\right] \\
& =i\left[a, a^{\dagger}\right] \\
& =i
\end{aligned}
$$

(It is worth mentioning that the other conventions (which we do not adopt here) defines $a=Q+i P$ and $a^{\dagger}=Q-i P$, and it will result in $[Q, P]=\frac{i}{2}$ instead, or 
$a=\frac{Q+i P}{2}$ to result in $[Q, P]=2 i$.)

\subsubsection{Three evolution pictures}

When the quantum system is evolving, depending on how we interpret it (either on the wave function or the operators or both), there are three different pictures: the Schrödinger picture, the Heisenberg picture and the interaction picture, also known as the Dirac picture.

\section{The Schrödinger picture}

In the Schrödinger picture, the wave function evolves with time but the operators are time-independent. Consider the Schrödinger equation with a Hamiltonian H:

$$
i \hbar \frac{d|\psi(t)\rangle}{d t}=H|\psi(t)\rangle .
$$

Plug the solution form $|\psi(t)\rangle=U\left(t, t_{0}\right)\left|\psi\left(t_{0}\right)\right\rangle$ into Schrödinger equation, get:

$$
i \hbar \frac{d U\left(t, t_{0}\right)}{d t}=H U\left(t, t_{0}\right)
$$

Integrate this and assume the Hamiltonian $H$ is time-independent, we get

$$
U\left(t, t_{0}\right)=e^{-i\left(t-t_{0}\right) H / \hbar}
$$

As the system evolves, at the moment of time $t$, the expectation value (observable 
value) of an operator $A_{0}$ becomes (here write $U\left(t, t_{0}\right)$ as $U$ for simplicity):

$$
\begin{aligned}
& \left\langle\psi_{S}(t)\left|A_{S}(t)\right| \psi_{S}(t)\right\rangle \\
= & \left\langle\psi(t)\left|A_{0}\right| \psi(t)\right\rangle \\
= & \left\langle\psi\left(t_{0}\right) U^{\dagger}\left|A_{0}\right| U \psi\left(t_{0}\right)\right\rangle
\end{aligned}
$$

In the Schrödinger picture, the wave function evolves as $\left|\psi_{S}(t)\right\rangle=|\psi(t)\rangle=$ $U\left(t, t_{0}\right)\left|\psi\left(t_{0}\right)\right\rangle$ and the operator doesn't change with time $A_{S}(t)=A_{0}$.

\section{The Heisenberg picture}

The Heisenberg picture assumes the wave function does not change but the operators will change over time. From Eq. (2.13) we can see that if we define $\left|\psi_{H}(t)\right\rangle=\left|\psi\left(t_{0}\right)\right\rangle$, and given that the matrix elements for the same operator $A$ should be the same no matter which picture it is in, we have:

$$
\begin{aligned}
& \left\langle\psi_{H}(t)\left|A_{H}(t)\right| \psi_{H}(t)\right\rangle \\
= & \left\langle\psi_{S}(t)\left|A_{S}(t)\right| \psi_{S}(t)\right\rangle \\
= & \left\langle\psi\left(t_{0}\right) U^{\dagger}\left|A_{0}\right| U \psi\left(t_{0}\right)\right\rangle \\
= & \left\langle\psi\left(t_{0}\right)\left|U^{\dagger} A_{0} U\right| \psi\left(t_{0}\right)\right\rangle
\end{aligned}
$$


Thus, in the Heisenberg picture, we have $\left|\psi_{H}(t)\right\rangle=\left|\psi\left(t_{0}\right)\right\rangle$ and $A_{H}(t)=U^{\dagger}\left(t, t_{0}\right) A_{0} U\left(t, t_{0}\right)$.

Taking the derivative of $A_{H}\left(t, t_{0}\right)$, knowing that $U\left(t, t_{0}\right)=e^{-i\left(t-t_{0}\right) H / \hbar}$ from Eq.(2.12), we have

$$
\begin{aligned}
\frac{d A_{H}}{d t} & =\frac{d\left(U^{\dagger}\left(t, t_{0}\right) A_{0} U\left(t, t_{0}\right)\right)}{d t} \\
& =\frac{1}{i \hbar}\left(-U^{\dagger} H A_{0} U+U^{\dagger} A_{0} H U\right) \\
& =\frac{1}{i \hbar}\left(-H U^{\dagger} A_{0} U+U^{\dagger} A_{0} U H\right) \\
& =\frac{1}{i \hbar}\left[A_{H}(t), H\right]
\end{aligned}
$$

Note in the above derivation, $[U, H]=0$ is used, and it is assumed $A_{0}$ is timeindependent.

So we obtain the Heisenberg equation

$$
\frac{d A_{H}(t)}{d t}=\frac{1}{i \hbar}\left[A_{H}(t), H\right]
$$

which describes the time evolution of the operator in the Heisenberg picture.

\section{The interaction picture}

Unlike the Schrödinger or Heisenberg picture which take either the operator or the wave function as time-independent, the interaction picture, also called the Dirac picture, assumes both the wave function and the operators are changing over the time. 
Consider the case of a Hamiltonian $H=H_{0}+V$, where the first term $H_{0}$ is a simple term typically single-system interaction (or a sum of single-system interactions), whereas the second term $V$ is typically a coupling term between different systems describing their interactions. $H_{0}$ and $V$ do not necessarily commute (actually in real cases they often don't).

There are different ways of defining the wave function and operators in the interaction picture, and here I will adopt the one that is used most in my research, especially the squeezing calculations, where the operators evolves solely under $V$ whereas the states evolve solely under $H_{0}$. No matter which definition we choose, once we have defined the operators (or wave function), the wave function (or operators) is determined correspondingly as well because the matrix elements of an operator need to be the same in all the pictures.

Now let us define the operator in the interaction picture as:

$$
A_{I}(t)=U_{0} A_{H}(t) U_{0}^{\dagger}=U_{0} U^{\dagger} A_{0} U U_{0}^{\dagger}
$$

Taking the time derivative,

$$
\begin{aligned}
\frac{d A_{I}(t)}{d t} & =\frac{d\left(U_{0} A_{H} U_{0}^{\dagger}\right)}{d t} \\
& =\frac{d U_{0}}{d t} A_{H} U_{0}^{\dagger}+U_{0} \frac{d A_{H}}{d t} U_{0}^{\dagger}+U_{0} A_{H} \frac{d U_{0}^{\dagger}}{d t} \\
& =-\frac{i}{\hbar}\left(H_{0} U_{0} A_{H} U_{0}^{\dagger}-U_{0} A_{H} U_{0}^{\dagger} H_{0}\right)+U_{0} \frac{d A_{H}}{d t} U_{0}^{\dagger} \\
& =\frac{i}{\hbar}\left[A_{I}, H_{0}\right]+U_{0} \frac{d A_{H}}{d t} U_{0}^{\dagger}
\end{aligned}
$$


Plug the Heisenberg equation $\frac{d A_{H}(t)}{d t}=\frac{1}{i \hbar}\left[A_{H}(t), H\right]$ into the above equation (Eq.(2.18)), the second term becomes,

$$
\begin{aligned}
U_{0} \frac{d A_{H}}{d t} U_{0}^{\dagger} & =\frac{1}{i \hbar} U_{0}\left[A_{H}, H\right] U_{0}^{\dagger} \\
& =-\frac{i}{\hbar} U_{0}\left(A_{H} H-H A_{H}\right) U_{0}^{\dagger} \\
& =-\frac{i}{\hbar} U_{0}\left(A_{H} H_{0}+A_{H} V-H_{0} A_{H}-V A_{H}\right) U_{0}^{\dagger} \\
& =-\frac{i}{\hbar}\left(U_{0} A_{H} U_{0}^{\dagger} H_{0}-H_{0} U_{0} A_{H} U_{0}^{\dagger}\right)-\frac{i}{\hbar}\left(U_{0} A_{H} V U_{0}^{\dagger}-U_{0} V A_{H} U_{0}^{\dagger}\right) \\
& =-\frac{i}{\hbar}\left[A_{I}, H_{0}\right]-\frac{i}{\hbar}\left(U_{0} A_{H} U_{0}^{\dagger} U_{0} V U_{0}^{\dagger}-U_{0} V U_{0}^{\dagger} U_{0} A_{H} U_{0}^{\dagger}\right) \\
& =-\frac{i}{\hbar}\left[A_{I}, H_{0}\right]-\frac{i}{\hbar}\left[A_{I}, V_{I}\right]
\end{aligned}
$$

Plug Eq.(2.19) into Eq. (2.18), we get:

$$
\frac{d A_{I}(t)}{d t}=-\frac{i}{\hbar}\left[A_{I}(t), V_{I}(t)\right]
$$

This is the evolution equation for the operators in the interaction picture, and it is important to notice that the operator evolution is independent of $H_{0}$, and that the $V_{I}(t)$ is in the interaction picture definition (i.e., not the Schrödinger picture V anymore). However, when we express everything in the interaction picture, the $V$ form stays the same. That is to say, if in the Schrödinger picture we have the form $V(A, B, C, \ldots)$, then its interaction picture counterpart $V_{I}(t)$ is simply $V\left(A_{I}, B_{I}, C_{I}, \ldots\right)$, where $A, B, C \ldots$ are various operators. 
More generally, if a function of operators has a certain expression in Schrödinger picture $f(A, B, C, \ldots)$, then if we convert every operators to the interaction picture, it has the same form as in the interaction picture $f\left(A_{I}, B_{I}, C_{I}, \ldots\right)=f_{I}(t)$. A quick proof for when the function has the general form $f(A, B, C, \ldots)=A^{n_{1}} B^{n_{2}} C^{n_{3}} \ldots$ is given as follows:

\section{Given}

$f(A, B, C)=A^{n_{1}} B^{n_{2}} C^{n_{3}}$

its interaction picture definition

$f_{I}(t)=U_{0} U^{\dagger}\left(A^{n_{1}} B^{n_{2}} C^{n_{3}}\right) U U_{0}^{\dagger}$.

On the other hand,

when we simply change the variable operators to the interaction form,

$$
\begin{aligned}
& f\left(A_{I}, B_{I}, C_{I}\right)=A_{I}^{n_{1}} B_{I}^{n_{2}} C_{I}^{n_{3}} \\
& =\left(U_{0} U^{\dagger} A U U_{0}^{\dagger}\right)^{n_{1}}\left(U_{0} U^{\dagger} B U U_{0}^{\dagger}\right)^{n_{2}}\left(U_{0} U^{\dagger} C U U_{0}^{\dagger}\right)^{n_{3}} \\
& =\underbrace{\left(U_{0} U^{\dagger} A U U_{0}^{\dagger}\right)\left(U_{0} U^{\dagger} A U U_{0}^{\dagger}\right) \ldots}_{n_{1}} \underbrace{\left(U_{0} U^{\dagger} B U U_{0}^{\dagger}\right)\left(U_{0} U^{\dagger} B U U_{0}^{\dagger}\right) \ldots}_{n_{2}} \underbrace{\left(U_{0} U^{\dagger} C U U_{0}^{\dagger}\right) \ldots}_{n_{3}} \\
& =U_{0} U^{\dagger}\left(A^{n_{1}} B^{n_{2}} C^{n_{3}}\right) U U_{0}^{\dagger} \\
& =f_{I}(t)
\end{aligned}
$$

The above only proved one type of the function expression in the power form, but similarly, if the function has the exponential form $f(A)=e^{A}$, to prove it we just need to expand the exponential in a power series and follow the above proof lines. Other 
forms can follow the same logic.

We will find this very useful when we deal with squeezing calculations later in this chapter, in there sometimes $V$ has the form $V=a a+a^{\dagger} a^{\dagger}$. When we work in the interaction picture, we simply have $V_{I}(t)=a_{I} a_{I}+a_{I}^{\dagger} a_{I}^{\dagger}$ and use the interaction picture operator evolution equation Eq.(2.20) for the operator $a_{I}(t)$ we do not need to worry about the free field term $H_{0}$ at all, which significantly simplifies the problem.

When the operators are defined as $A_{I}(t)=U_{0} U^{\dagger} A_{0} U U_{0}^{\dagger}$, the wave function should be as follows to preserve the matrix elements:

$$
\left|\psi_{I}(t)\right\rangle=U_{0}\left|\psi\left(t_{0}\right)\right\rangle
$$

And its derivative can be obtained after simple math:

$$
\begin{aligned}
\frac{d\left|\psi_{I}(t)\right\rangle}{d t} & =\frac{d\left[U_{0}\left|\psi\left(t_{0}\right)\right\rangle\right]}{d t} \\
& =\frac{d U_{0}}{d t}\left|\psi\left(t_{0}\right)\right\rangle \\
& =-\frac{i}{\hbar} H_{0}\left|\psi\left(t_{0}\right)\right\rangle
\end{aligned}
$$

Now the three pictures are all covered. I use a table to summarize and compare them: 
Table 2.1: Three pictures to describe the system evolution

\begin{tabular}{cccc}
\hline \hline Definition & Schrödinger & Heisenberg & Interaction \\
\hline$|\psi\rangle=$ & $U\left|\psi\left(t_{0}\right)\right\rangle$ & $\left|\psi\left(t_{0}\right)\right\rangle$ & $U_{0}\left|\psi\left(t_{0}\right)\right\rangle$ \\
$\frac{d|\psi\rangle}{d t}=$ & $\frac{1}{i \hbar} H|\psi(t)\rangle$ & 0 & $\frac{1}{i \hbar} H_{0}\left|\psi\left(t_{0}\right)\right\rangle$ \\
$A=$ & $A_{0}$ & $U^{\dagger} A_{0} U$ & $U_{0} U^{\dagger} A_{0} U U_{0}^{\dagger}$ \\
$\frac{d A}{d t}=$ & 0 & $-\frac{i}{\hbar}\left[A_{H}(t), H\right]$ & $-\frac{i}{\hbar}\left[A_{I}(t), V_{I}\right]$ \\
\hline
\end{tabular}

The wave function $(|\psi\rangle)$, evolution of wave function $\left(\frac{d|\psi\rangle}{d t}\right)$, operator $(A)$, and evolution of operator $\left(\frac{d A}{d t}\right)$ in three different pictures. 


\subsection{The beam splitter}

The beam splitter is a very important element of quantum optics, both in the experimental and theoretical sense. It has very simple structure - two inputs and two outputs, but it contains crucial quantum optics concepts. For example, in quantum optics, because "vacuum" is everywhere, even when one input of the beam splitter is "empty" and has "nothing" sent into, we need to consider the vacuum mode being there. Also, the Hong-Ou-Mandel effect [49] is a very good example of the quantum optics effect of the beam splitter. It is a two-photon interference where two identical single-photon waves enter a $50: 50$ beam splitter, one in each input port, and they always both leave from the same output. In this section I will not go into details of those examples, but I will outline the derivation of the beam splitter matrix and its properties.

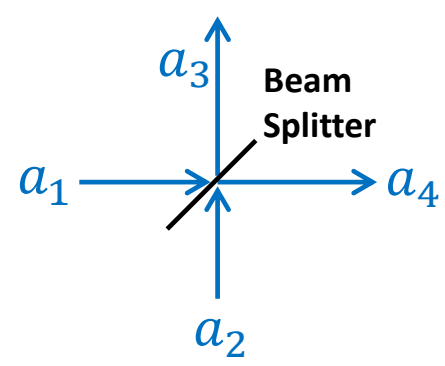

Figure 2.1: Beam Splitter.

Figure 2.1 shows a beam splitter and its inputs and outputs. The output modes 
are related to the input ones as:

$$
\left(\begin{array}{l}
a_{3} \\
a_{4}
\end{array}\right)=\left(\begin{array}{ll}
R_{31} & T_{32} \\
T_{41} & R_{42}
\end{array}\right)\left(\begin{array}{l}
a_{1} \\
a_{2}
\end{array}\right)
$$

This is the beam splitter matrix, from which we have:

$$
\begin{aligned}
& a_{3}=R_{31} a_{1}+T_{32} a_{2} \\
& a_{3}^{\dagger}=R_{31}^{*} a_{1}^{\dagger}+T_{32}^{*} a_{2}^{\dagger} \\
& a_{4}=T_{41} a_{1}+R_{42} a_{2} \\
& a_{4}^{\dagger}=T_{41}^{*} a_{1}^{\dagger}+R_{42}^{*} a_{2}^{\dagger}
\end{aligned}
$$

The photon number conservation (energy conservation) for a lossless beam splitter requires that $a_{1}^{\dagger} a_{1}+a_{2}^{\dagger} a_{2}=a_{3}^{\dagger} a_{3}+a_{4}^{\dagger} a_{4}$, which yields:

$$
\left\{\begin{array}{l}
\left|R_{31}\right|^{2}+\left|T_{41}\right|^{2}=1 \\
\left|T_{32}\right|^{2}+\left|R_{42}\right|^{2}=1 \\
R_{31}^{*} T_{32}^{*}+T_{41}^{*} R_{42}^{*}=0
\end{array}\right.
$$

Now we separate the beam splitter parameters' amplitudes and phases and write them as $R_{31}=\left|R_{31}\right| e^{i \phi_{31}}, T_{32}=\left|T_{32}\right| e^{i \phi_{32}}, T_{41}=\left|T_{41}\right| e^{i \phi_{41}}$ and $R_{42}=\left|R_{42}\right| e^{i \phi_{42}}$. Plug them in the Eq.(2.26), we can get

$$
\left\{\begin{array}{l}
\left|R_{31}\right|=\left|R_{42}\right| \\
\left|T_{32}\right|=\left|T_{41}\right| \\
\phi_{32}-\phi_{31}=\phi_{42}-\phi_{41}+(2 n+1) \pi
\end{array}\right.
$$


To satisfy the relations in Eq.(2.27), there are many different ways of choosing the four parameters. Two common ways of definitions are to choose $\left|R_{31}\right|=\left|R_{42}\right|=\rho$, $\left|T_{32}\right|=\left|T_{41}\right|=\tau, \phi_{31}=\phi_{32}=\phi_{41}=0, \phi_{42}=\pi ;$ or $\left|R_{31}\right|=\left|R_{42}\right|=\rho,\left|T_{32}\right|=\left|T_{41}\right|=$ $\tau, \phi_{31}=\phi_{42}=0, \phi_{32}=\phi_{41}=\pi / 2$. Those two choices yields, respectively

$$
\left(\begin{array}{cc}
\rho & \tau \\
\tau & -\rho
\end{array}\right) \quad \text { or } \quad\left(\begin{array}{cc}
\rho & i \tau \\
i \tau & \rho
\end{array}\right)
$$

These conventions merely correspond to phase shifts at the input and output ports of the beam splitter. 


\subsection{Quantum states}

In this section I will talk about a few most commonly used quantum optical states:

Fock state, coherent state and squeezing state.

\subsubsection{Fock state}

Fock state, also called photon number state, is the eigenstate of harmonic oscillator Hamiltonian $H$ and the photon number operator $N$.

In the Dirac notation it is represented as $|n\rangle$.

The creation and annihilation operators implement on the Fock state as follows:

$$
\begin{aligned}
& a^{\dagger}|n\rangle=\sqrt{n+1}|n+1\rangle \\
& a|n\rangle=\sqrt{n}|n-1\rangle
\end{aligned}
$$

It is straightforward to see that the Fock state is the eigenstate of the photon number operator $N$ and harmonic oscillator Hamiltonian $H$.

$$
\begin{aligned}
& N|n\rangle=a^{\dagger} a|n\rangle=n|n\rangle \\
& H|n\rangle=\left(N+\frac{1}{2}\right)|n\rangle=\left(n+\frac{1}{2}\right)|n\rangle
\end{aligned}
$$

Since the eigenstates of the Hamiltonian form an orthogonal basis, the Fock states must satisfy:

$$
\left\langle n \mid n^{\prime}\right\rangle=\delta_{n n^{\prime}}
$$


where $\delta_{n n^{\prime}}$ is the Kronecker delta function defined as:

$$
\begin{aligned}
& \delta_{n n^{\prime}}=1, \text { if } n=n^{\prime} ; \\
& \delta_{n n^{\prime}}=0, \text { if } n \neq n^{\prime}
\end{aligned}
$$

Now let us calculate the photon number derivation of the Fock state:

$$
\begin{aligned}
& \bar{N}=\langle n|N| n\rangle=N \\
& \overline{N^{2}}=\left\langle n\left|N^{2}\right| n\right\rangle=N^{2} \\
& \Delta N=\sqrt{\left|\overline{N^{2}}-(\bar{N})^{2}\right|}=0
\end{aligned}
$$

This makes sense intuitively - we know it should be $\Delta N=0$ for Fock state. Because Fock state is the eigenstate of the photon number operator, so for a certain Fock state $|n\rangle$, there should be only one particular photon number associated with it based on the definition of eigenstate. So there should be no ambiguous in the photon number for a given Fock state, thus, the photon number derivation should be zero.

Notice that the vacuum state is also a Fock state, with photon number 0 . Thus, to generate Fock state $|n\rangle$ from the vacuum state $|0\rangle$, we need to repeatedly use creation operator $n$ times to raise the photon number from 0 to $n$, together with the consideration of the normalization factor:

$$
\begin{aligned}
& a^{\dagger^{n}}|0\rangle=\sqrt{n !}|n\rangle \\
& \frac{a^{\dagger^{n}}}{\sqrt{n !}}|0\rangle=|n\rangle
\end{aligned}
$$

So the operator that generate a Fock state $|n\rangle$ out of a vacuum state is $\frac{a^{\dagger}}{\sqrt{n !}}$. 
The expectation values of the quadratures $Q$ and $P$ for the Fock state are:

$$
\begin{aligned}
& \bar{Q}=\langle n|Q| n\rangle=\frac{1}{\sqrt{2}}\left\langle n\left|a+a^{\dagger}\right| n\right\rangle=0 \\
& \bar{P}=\langle n|P| n\rangle=-\frac{i}{\sqrt{2}}\left\langle n\left|a-a^{\dagger}\right| n\right\rangle=0
\end{aligned}
$$

Also we have that:

$$
\begin{aligned}
& \overline{Q^{2}}=\left\langle n\left|Q^{2}\right| n\right\rangle=\frac{1}{2}\left\langle n\left|\left(a+a^{\dagger}\right)^{2}\right| n\right\rangle=\frac{1}{2}\left\langle n\left|a a^{\dagger}+a^{\dagger} a\right| n\right\rangle=n+\frac{1}{2} \\
& \overline{P^{2}}=\left\langle n\left|P^{2}\right| n\right\rangle=-\frac{1}{2}\left\langle n\left|\left(a-a^{\dagger}\right)^{2}\right| n\right\rangle=-\frac{1}{2}\left\langle n\left|-a a^{\dagger}-a^{\dagger} a\right| n\right\rangle=n+\frac{1}{2}
\end{aligned}
$$

So the derivations in $Q$ and $P$ are:

$$
\begin{aligned}
& \Delta Q=\sqrt{\left|\overline{Q^{2}}-\bar{Q}^{2}\right|}=\sqrt{n+\frac{1}{2}} \\
& \Delta P=\sqrt{\left|\overline{P^{2}}-\bar{P}^{2}\right|}=\sqrt{n+\frac{1}{2}}
\end{aligned}
$$

The derivations in $Q$ and $P$ will increase as the photon number $n$ increases, and the derivations do not depend on the quadrature angle, so the Fock state have polar symmetry with respect to the origin in the phase space. Notice when $n=0, \Delta Q=$ $\Delta P=\frac{1}{\sqrt{2}}$, which is the case for vacuum state. 


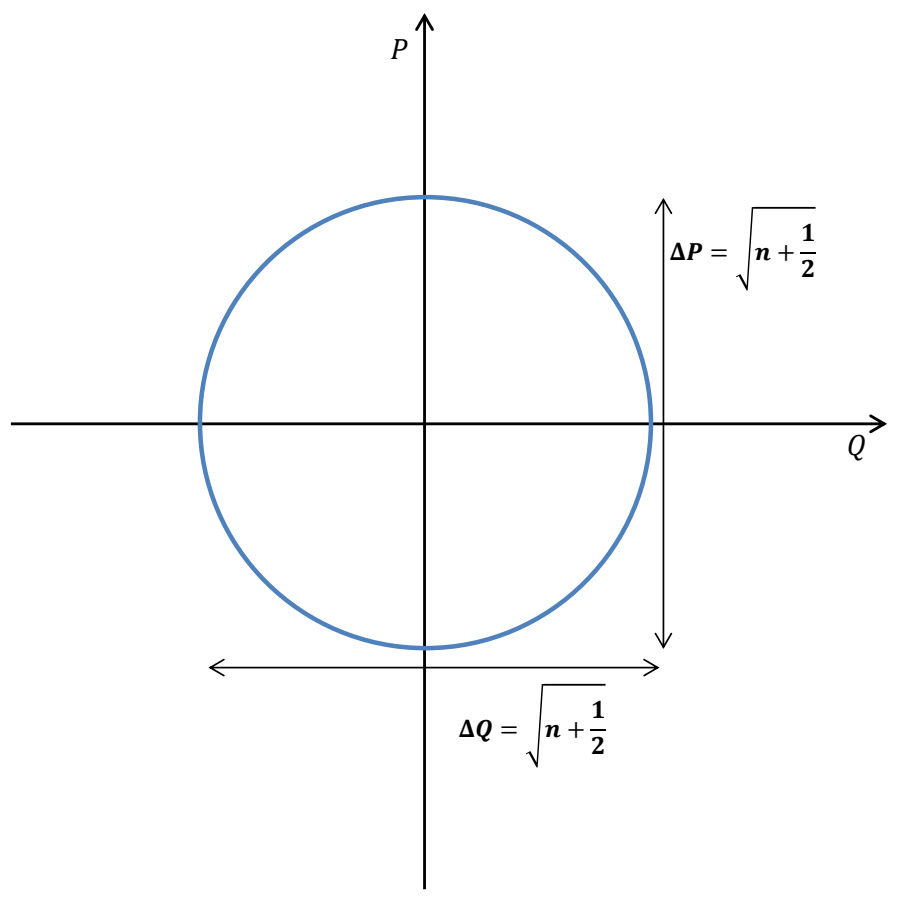

Figure 2.2: Phasor diagram for the Fock state. The amplitude (radius) is perfectly defined, but the phase is uncertain. The standard deviations of the quadratures $Q$ and $P$ increase as the photon number $n$ increases. 


\subsubsection{Coherent state}

The quantum-mechanical equivalent of a classical monochromatic electromagnetic wave is called a coherent state. Stabilized emissions well above threshold are good sources of coherent states.

Vacuum state $|0\rangle$ is the only state that is both coherent state $(\alpha=0)$ and Fock state $(n=0)$.

Coherent states are represented in the Dirac notation as $|\alpha\rangle$, where $\alpha$ is a dimensionless complex number.

$$
\alpha=|\alpha| e^{i \phi}
$$

The coherent state is obtained by displacing the vacuum state:

$$
\begin{aligned}
|\alpha\rangle & =D(\alpha)|0\rangle \\
& =e^{\alpha a^{\dagger}-\alpha^{*} a}|0\rangle \\
& =e^{\alpha a^{\dagger}} e^{-\alpha^{*} a} e^{-\frac{|\alpha|^{2}}{2}}|0\rangle \\
& =\left[1+\left(\alpha a^{\dagger}\right)+\frac{\left(\alpha a^{\dagger}\right)^{2}}{2 !}+\ldots\right]\left[1+\left(-\alpha^{*} a\right)+\frac{\left(-\alpha^{*} a\right)^{2}}{2 !}+\ldots\right] e^{-\frac{|\alpha|^{2}}{2}}|0\rangle \\
& =e^{-\frac{|\alpha|^{2}}{2}} \sum_{n=0}^{\infty} \frac{\alpha^{n}}{n !}\left(a^{\dagger}\right)^{n}|0\rangle \\
& =e^{-\frac{|\alpha|^{2}}{2}} \sum_{n=0}^{\infty} \frac{\alpha^{n}}{\sqrt{n !}}|n\rangle
\end{aligned}
$$

In the above derivation an important formula for exponential calculation with operators was used-"Baker-Campbell-Hausdorff formula", shown as follows: 


$$
\begin{aligned}
& e^{\mathbf{A}+\mathbf{B}}=e^{\mathbf{A}} e^{\mathbf{B}} e^{-\frac{[\mathbf{A}, \mathbf{B}]}{2}} \\
& \text { when both } \mathbf{A} \text { and } \mathbf{B} \text { commute with their commutator. }
\end{aligned}
$$

It is easy to see that

$$
a|\alpha\rangle=\alpha|\alpha\rangle .
$$

The expectation value of the photon number and its standard deviation is

$$
\begin{aligned}
& \bar{N}=\langle\alpha|N| \alpha\rangle=\left\langle\alpha\left|a^{\dagger} a\right| \alpha\right\rangle=|\alpha|^{2} \\
& \overline{N^{2}}=\left\langle\alpha\left|a^{\dagger} a a^{\dagger} a\right| \alpha\right\rangle=\left\langle\alpha\left|a^{\dagger}\left(a^{\dagger} a+1\right) a\right| \alpha\right\rangle \\
& =|\alpha|^{4}+|\alpha|^{2} \\
& \Delta N=\sqrt{\left|\overline{N^{2}}-\bar{N}^{2}\right|}=\sqrt{\left.|| \alpha\right|^{4}+|\alpha|^{2}-|\alpha|^{4} \mid}=|\alpha|=\sqrt{\bar{N}}
\end{aligned}
$$

The expectation values of the quadratures $Q$ and $P$ (defined as in Eq.(2.6)) are:

$$
\begin{aligned}
& \bar{Q}=\langle\alpha|Q| \alpha\rangle=\frac{1}{\sqrt{2}}\left\langle\alpha\left|a+a^{\dagger}\right| \alpha\right\rangle=\frac{1}{\sqrt{2}}\left(\alpha+\alpha^{*}\right) \\
& \overline{Q^{2}}=\left\langle\alpha\left|Q^{2}\right| \alpha\right\rangle=\frac{1}{2}\left\langle\alpha\left|a^{2}+a^{\dagger^{2}}+2 a^{\dagger} a-1\right| \alpha\right\rangle=\frac{1}{2}\left(\alpha^{2}+\alpha^{* 2}+2|\alpha|^{2}-1\right) \\
& \bar{P}=\langle\alpha|P| \alpha\rangle=\frac{-i}{\sqrt{2}}\left\langle\alpha\left|a-a^{\dagger}\right| \alpha\right\rangle=\frac{-i}{\sqrt{2}}\left(\alpha-\alpha^{*}\right) \\
& \overline{P^{2}}=\left\langle\alpha\left|P^{2}\right| \alpha\right\rangle=-\frac{1}{2}\left\langle\alpha\left|a^{2}+a^{\dagger^{2}}-2 a^{\dagger} a+1\right| \alpha\right\rangle=-\frac{1}{2}\left(\alpha^{2}+\alpha^{* 2}-2|\alpha|^{2}+1\right)
\end{aligned}
$$

Their standard deviations are:

$$
\begin{aligned}
& \Delta Q=\sqrt{\overline{Q^{2}}-\bar{Q}^{2}}=\frac{1}{\sqrt{2}} \\
& \Delta P=\sqrt{\overline{P^{2}}-\bar{P}^{2}}=\frac{1}{\sqrt{2}}
\end{aligned}
$$


From this we can see the coherent state is a minimum uncertainty state. There is no intrinsic preference to either of the two quadratures, like the vacuum state. Coherent state can be considered as displaced vacuum state, with the uncertainty circle of the vacuum displaced from the original by the field vector $\alpha$ of the coherent state. Figure 2.3 shows a coherent state on the phase diagram. From this figure we can see many parameters and properties of coherent states as we derived above.

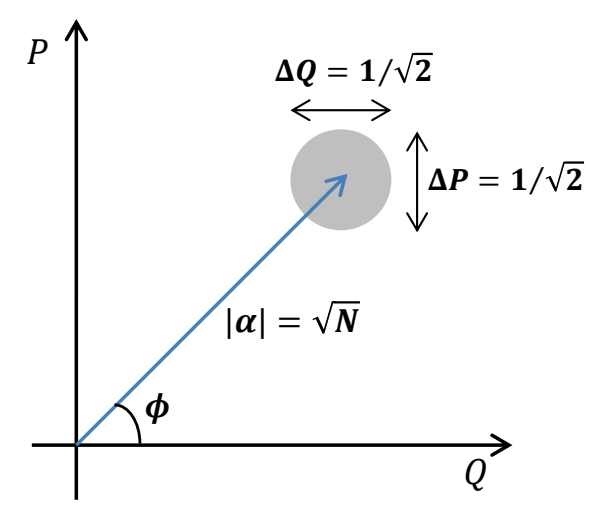

Figure 2.3: Phasor diagram for the coherent state. It is displaced $\alpha$ from the origin (vacuum) and the gray "fuzzy ball" at the end of the displacement shows the uncertainties in $Q, P$ and all the directions are equal. The length of the displacement is also the square root of the average photon number.

\subsubsection{Squeezed state}

In the previous subsection we talked about coherent state, where the state in the phasor diagram looks like a "fuzzy ball" whose symmetric diameter shows that $\Delta Q=$ $\Delta P=1 / \sqrt{2}$, satisfying the minimum requirement of the uncertainty principle $\Delta Q \Delta P \geq$ $|[Q, P]| / 2=1 / 2$. In this subsection we will talk about a different kind of quantum state, squeezed state, also satisfy the uncertainty principle, but has unequal distribu- 
tion in the standard deviations of the two quadratures.

The generation and Hamiltonian development of the squeezed state will be covered in the next section, and right now we just study the properties of the squeezed state itself, in particular, the vacuum squeezed state.

The squeezed vacuum state is obtained by squeezing the vacuum:

$$
|0, \varepsilon\rangle=S(\varepsilon)|0\rangle=e^{\frac{1}{2}\left(\varepsilon a^{\dagger^{2}}-\varepsilon^{*} a^{2}\right)}|0\rangle
$$

where $S(\varepsilon)$ is the squeezing operator and $\varepsilon=r e^{i \psi} . r=\chi \beta t$ is the squeezing parameter and $\psi$ is the nonlinear phase $\psi=\varphi_{b}-2 \varphi_{a}$.

We will derive in next section the Bogoliubov transformation from solving Heisenberg equation in the interaction picture. Here we just show the results:

\section{Bogoliubov transformation:}

$$
\begin{aligned}
& a(t)=S^{\dagger}(\varepsilon) a S(\varepsilon)=a \cosh r+a^{\dagger} e^{i \psi} \sinh r \\
& a^{\dagger}(t)=S^{\dagger}(\varepsilon) a^{\dagger} S(\varepsilon)=a^{\dagger} \cosh r+a e^{-i \psi} \sinh r
\end{aligned}
$$

In the following we will calculate the expectation value and standard deviation of the photon number of a squeezed state using Bogoliubov transformation.

$$
\begin{aligned}
\bar{N} & =\langle 0, \varepsilon|N| 0, \varepsilon\rangle \\
& =\left\langle 0\left|\left[S^{\dagger}(\varepsilon) a^{\dagger} S(\varepsilon)\right]\left[S^{\dagger}(\varepsilon) a S(\varepsilon)\right]\right| 0\right\rangle \\
& =\left\langle 0\left|\left(a^{\dagger} \cosh r+a e^{-i \psi} \sinh r\right)\left(a \cosh r+a^{\dagger} e^{i \psi} \sinh r\right)\right| 0\right\rangle \\
& =\sinh ^{2} r
\end{aligned}
$$




$$
\begin{aligned}
\overline{N^{2}} & =\left\langle 0, \varepsilon\left|N^{2}\right| 0, \varepsilon\right\rangle \\
& =\left\langle 0\left|\left\{\left[S^{\dagger}(\varepsilon) a^{\dagger} S(\varepsilon)\right]\left[S^{\dagger}(\varepsilon) a S(\varepsilon)\right]\right\}^{2}\right| 0\right\rangle \\
& =\left\langle 0\left|\left[\left(a^{\dagger} \cosh r+a e^{-i \psi} \sinh r\right)\left(a \cosh r+a^{\dagger} e^{i \psi} \sinh r\right)\right]^{2}\right| 0\right\rangle \\
& =\sinh ^{2} r\left\langle 0\left|a\left(a \cosh r+a^{\dagger} e^{i \psi} \sinh r\right)\left(a^{\dagger} \cosh r+a e^{-i \psi} \sinh r\right) a^{\dagger}\right| 0\right\rangle \\
& =\sinh ^{2} r\left\langle 1\left|\left(a \cosh r+a^{\dagger} e^{i \psi} \sinh r\right)\left(a^{\dagger} \cosh r+a e^{-i \psi} \sinh r\right)\right| 1\right\rangle \\
& =\sinh ^{2} r\left\langle 1\left|a a^{\dagger} \cosh ^{2} r+a^{\dagger} a e^{i \psi} \sinh ^{2} r\right| 1\right\rangle \\
& =\sinh ^{2} r\left(2 \cosh ^{2} r+\sinh ^{2} r\right) \\
& =2 \sinh ^{2} r \cosh ^{2} r+\sinh ^{4} r
\end{aligned}
$$

The standard deviation of the photon number is:

$$
\Delta N=\sqrt{\left|\overline{N^{2}}-\bar{N}^{2}\right|}=\sqrt{2}|\sinh r \cosh r|=\frac{1}{\sqrt{2}}|\sinh 2 r|
$$

Notice that the standard deviation of the photon number for a squeezed state is independent of the squeezing angle, only dependent on the squeezing parameter $r$. And the expectation value of the photon number is not nonzero, showing a squeezed vacuum state is not empty of photons, which makes sense since one of the means of squeezing is to use two-photon emission in a nonlinear crystal.

To look at the quadrature's expectation values and standard deviation, let us 
define the generalized quadrature $A_{\theta}$ :

$$
A_{\theta}=\frac{1}{\sqrt{2}}\left(e^{-i \theta} a+e^{i \theta} a^{\dagger}\right)
$$

We can easily see that $Q$ and $P$ correspond to $A_{\theta=0}$ and $A_{\theta=\pi / 2}$.

The expectation value of the generalized quadrature is:

$$
\begin{aligned}
& \overline{A_{\theta}}=\left\langle 0, \varepsilon\left|A_{\theta}\right| 0, \varepsilon\right\rangle \\
& =\frac{1}{\sqrt{2}}\left\langle 0\left|S^{\dagger}(\varepsilon)\left(e^{-i \theta} a+e^{i \theta} a^{\dagger}\right) S(\varepsilon)\right| 0\right\rangle \\
& =\frac{1}{\sqrt{2}}\left\langle 0\left|e^{-i \theta}\left(a \cosh r+a^{\dagger} e^{i \psi} \sinh r\right)+e^{i \theta}\left(a^{\dagger} \cosh r+a e^{-i \psi} \sinh r\right)\right| 0\right\rangle \\
& =0
\end{aligned}
$$

The expectation value of $A_{\theta}^{2}$ is

$$
\begin{aligned}
\overline{A_{\theta}^{2}} & =\frac{1}{2}\left\langle 0, \varepsilon\left|A_{\theta}^{2}\right| 0, \varepsilon\right\rangle \\
= & \frac{1}{2}\left\langle 0\left|S^{\dagger}(\varepsilon)\left(e^{-i \theta} a+e^{i \theta} a^{\dagger}\right)^{2} S(\varepsilon)\right| 0\right\rangle \\
= & \frac{1}{2}\left\langle 0\left|\left[e^{-i \theta}\left(a \cosh r+a^{\dagger} e^{i \psi} \sinh r\right)+e^{i \theta}\left(a^{\dagger} \cosh r+a e^{-i \psi} \sinh r\right)\right]^{2}\right| 0\right\rangle \\
= & \frac{1}{2}\langle 0| a a^{\dagger}\left(e^{-2 i \theta} \cosh r e^{i \psi} \sinh r+e^{2 i \theta} \cosh r e^{-i \psi} \sinh r+e^{-i \theta+i \theta} \cosh ^{2} r\right. \\
& \left.+e^{-i \theta+i \theta} \sinh ^{2} r\right)|0\rangle \\
= & \frac{1}{2}[\cos (2 \theta-\psi) \sinh 2 r+\cosh 2 r]
\end{aligned}
$$

So the standard deviation of the generalized quadrature is:

$$
\Delta A_{\theta}=\sqrt{\left|\overline{A_{\theta}^{2}}-{\overline{A_{\theta}}}^{2}\right|}=\frac{1}{\sqrt{2}} \sqrt{\cos (2 \theta-\psi) \sinh 2 r+\cosh 2 r}
$$

It is easy to see that when $\theta=\psi / 2, \Delta A_{\theta}=e^{r} / \sqrt{2}$; when $\theta=(\psi+\pi) / 2$, 
$\Delta A_{\theta}=e^{-r} / \sqrt{2}$, which is smaller than the shot noise limit (i.e. the vacuum case) $\Delta A_{\theta}($ vaccum $)=1 / \sqrt{2}$. Those two cases are the antisqueezing and squeezing cases respectively. Thus, the squeezing angle $\psi$ determines which quadrature is squeezed and which is antisqueezed.

In particular, when $\psi=0$, we have $\Delta Q=e^{r} / \sqrt{2}$ and $\Delta P=e^{-r} / \sqrt{2}$. This is shown in Figure 2.4:

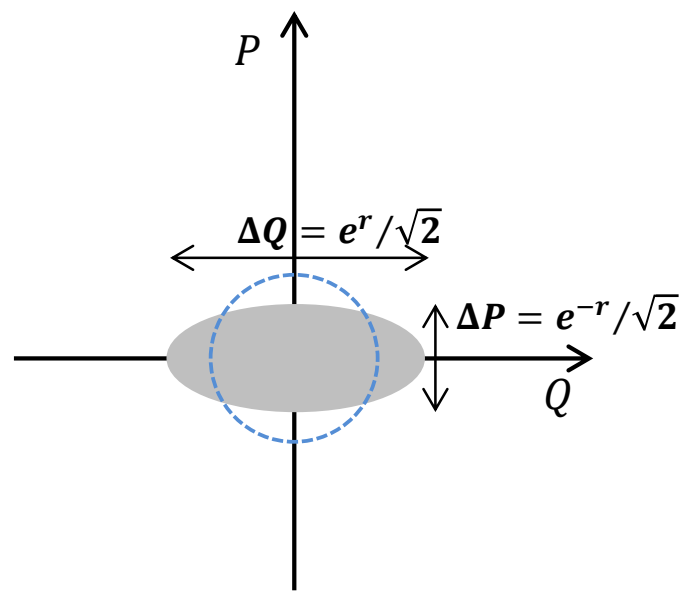

Figure 2.4: Phasor diagram for the vacuum squeezed state. The grey ball shows the vacuum squeezed state, while the dashed blue circle shows the outline of the vacuum state. The squeezed state the standard derivation $\Delta P$ is smaller than that of the vacuum state, and $\Delta Q$ is bigger than that of the vacuum state. But their product is the same as that of the vacuum $\Delta Q \Delta P=1 / 2$, and this indicates these two shapes have the same area. 


\subsection{Squeezing generation}

In this section we will talk about how to generate squeezed states from 3 -wave mixing nonlinear process.

\subsubsection{Single-mode squeezing}

We consider a case of nonlinear interaction which annihilates a pump photon of frequency $2 \omega$ and creates a pair of single photons at frequency $\omega$. The Hamiltonian is:

$$
\begin{aligned}
H & =H_{0}+V \\
& =\hbar \omega\left(a^{\dagger} a+2 b^{\dagger} b\right)+i \hbar \frac{\chi}{2}\left(b a^{\dagger^{2}}-b^{\dagger} a^{2}\right)
\end{aligned}
$$

where the first part corresponds to the free field evolution for both fields and the second part corresponds to the parametric down conversion nonlinear process where a pump photon $b$ is annihilated and two degenerate down converting photons at half frequency of that of the pump were created $a^{\dagger}$.

Here using interaction picture (details see 2.2.2) is the best. Changing all the operators to the interaction picture (add subscript "I") and then Hamiltonian becomes that of the interaction picture as well:

$$
\begin{aligned}
H_{I} & =H_{0 I}+V_{I} \\
& =\hbar \omega\left(a_{I}^{\dagger} a_{I}+2 b_{I}^{\dagger} b_{I}\right)+i \hbar \frac{\chi}{2}\left(b_{I} a_{I}^{\dagger^{2}}-b_{I}^{\dagger} a_{I}^{2}\right)
\end{aligned}
$$

The evolution equation for the operators (Eq.(2.20)) in the interaction picture is

$$
\frac{d A_{I}(t)}{d t}=-\frac{i}{\hbar}\left[A_{I}(t), V_{I}(t)\right]
$$


Assume the pump is classical, so we have $b \rightarrow \beta$, indicating the pump field $b$ is in coherent state $|\beta\rangle$.

The evolution equation for operators $a_{I}$ and $a_{I}^{\dagger}$ are:

$$
\begin{aligned}
& \dot{a}_{I}(t)=-\frac{i}{\hbar}\left[a_{I}, V_{I}\right]=\frac{\chi \beta}{2}\left[a_{I}, a_{I}^{\dagger}\right]=\chi \beta a_{I}^{\dagger}(t) \\
& \dot{a}_{I}^{\dagger}(t)=-\frac{i}{\hbar}\left[a_{I}^{\dagger}, V_{I}\right]=-\frac{\chi \beta^{*}}{2}\left[a_{I}^{\dagger}, a_{I}^{2}\right]=\chi \beta^{*} a_{I}(t)
\end{aligned}
$$

From this point on and for the rest of this section, we will work in the interaction picture and for simplicity, the subscript "I" will be omitted. Also, define $\kappa \equiv \chi \beta$, the above equations simplify to:

$$
\begin{aligned}
& \dot{a}(t)=\kappa a^{\dagger}(t) \\
& \dot{a}^{\dagger}(t)=\kappa^{*} a(t)
\end{aligned}
$$

From Eq.(2.58), we have:

$$
\begin{aligned}
& \ddot{a}(t)=\kappa \dot{a}^{\dagger}(t)=|\kappa|^{2} a \\
\Rightarrow & \ddot{a}(t)-|\kappa|^{2} a(t)=0
\end{aligned}
$$

This is a second order derivative equation, and the characteristic equation for it is:

$$
a(t)=a_{+} e^{|\kappa| t}+a_{-} e^{-|\kappa| t}
$$

Plug this in the initial conditions:

$$
\left\{\begin{array}{l}
a(t=0)=a=a_{+}+a_{-} \\
\dot{a}(t=0)=\kappa a^{\dagger}=|\kappa|\left(a_{+}-a_{-}\right)
\end{array}\right.
$$


which yields

$$
\left\{\begin{array}{l}
a_{+}=\frac{1}{2}\left(a+\frac{\kappa}{|\kappa|} a^{\dagger}\right) \\
a_{-}=\frac{1}{2}\left(a-\frac{\kappa}{|\kappa|} a^{\dagger}\right)
\end{array}\right.
$$

Look at the phase of $\kappa, \kappa=\chi \beta=\chi|\beta| e^{i \psi}$ ( $\psi$ is the phase of the pump). Define $r \equiv|\kappa| t$, we the solution $a(t)$ is:

$$
a=(r)=\frac{1}{2}\left[\left(a+e^{i \psi} a^{\dagger}\right) e^{r}+\left(a-e^{i \psi} a^{\dagger}\right) e^{-r}\right]
$$

We can rewrite the above equation as:

$$
a_{I}(r)=a \cosh r+e^{i \psi} a^{\dagger} \sinh r
$$

This is called Bogoliubov Transformation, an important operator evolution in the interaction picture after squeezing.

With this relation, we can calculate the expectation values and standard deviations of different operators that can be expressed in the form of $a$ and $a^{\dagger}$ for the squeezed state. When we are dealing with vacuum squeezing (where the initial state is $|0\rangle$ ), it is especially very simple, because in the interaction picture,

$$
\left|\psi_{I}\right\rangle=U_{0}|0\rangle=e^{-i \omega t a^{\dagger} a}|0\rangle=|0\rangle
$$

So an expectation value of an operator $O$ in the interaction picture for the case 
where the initial state is vacuum is:

$$
\begin{aligned}
\bar{O} & =\left\langle\psi_{I}\left|O_{I}\right| \psi_{I}\right\rangle \\
& =\left\langle 0 U_{0}^{\dagger}\left|O_{I}\right| U_{0} 0\right\rangle \\
& =\left\langle 0\left|O_{I}\right| 0\right\rangle
\end{aligned}
$$

\subsubsection{Two-mode squeezing}

This section we will look briefly at the two mode squeezing case.

Similar as the single-mode squeezing case, we work in the interaction picture and neglect the free field evolution part $H_{0}$, we also assume the pump field is classic $\beta$, and that $\kappa \equiv \chi \beta=\chi|\beta| e^{i \psi}$. However, unlike the single-mode squeezing, for two-mode squeezing, the parametric down conversion process is nondegenerate, so we will have two quantum fields, $a_{1}$ and $a_{2}$ respectively.

Again, all of the following work in this section are in the interaction picture and the subscript $I$ is omitted.

$$
V=i \hbar\left(\kappa a_{1}^{\dagger} a_{2}^{\dagger}-\kappa^{*} a_{1} a_{2}\right)
$$

Solve the evolution equations for the creation and annihilation operators in the 
interaction picture (Eq.(2.20)), we obtain two coupled equations:

$$
\begin{aligned}
& \dot{a}_{1}(t)=\kappa a_{2}^{\dagger}(t) \\
& {\dot{a_{1}}}^{\dagger}(t)=\kappa^{*} a_{2}(t) \\
& \dot{a}_{2}(t)=\kappa a_{1}^{\dagger}(t) \\
& {\dot{a_{2}}}^{\dagger}(t)=\kappa^{*} a_{1}(t)
\end{aligned}
$$

From them we can get that:

$$
\begin{aligned}
& \ddot{a}_{1}(t)=\kappa \dot{a}_{2}^{\dagger}(t)=|\kappa|^{2} a_{1}(t) \\
& \ddot{a}_{2}(t)=\kappa \dot{a}_{1}^{\dagger}(t)=|\kappa|^{2} a_{2}(t)
\end{aligned}
$$

The characteristic equations for the solution of Eq.(2.69) are:

$$
\begin{aligned}
& a_{1}(t)=a_{1+} e^{|\kappa| t}+a_{1-} e^{-|\kappa| t} \\
& a_{2}(t)=a_{2+} e^{|\kappa| t}+a_{2-} e^{-|\kappa| t}
\end{aligned}
$$

where $a_{1+}, a_{1-}, a_{2+}, a_{2-}$ are time-independent parameters. Use the initial conditions

$$
\begin{aligned}
& a_{1}(t=0)=a_{1}=a_{1+}+a_{1-} \\
& a_{1}(t=0)=\kappa a_{2}^{\dagger}=|\kappa|\left(a_{1+}-a_{1-}\right) \\
& a_{2}(t=0)=a_{2}=a_{2+}+a_{2-} \\
& a_{2}(t=0)=\kappa a_{1}^{\dagger}=|\kappa|\left(a_{2+}-a_{2-}\right)
\end{aligned}
$$

Solve for the parameters $a_{1+}, a_{1-}, a_{2+}, a_{2-}$, we obtain the evolution equations of op- 
erators $a_{1}(t)$ and $a_{2}(t)$ (as before define $r \equiv \kappa t$ ):

$$
\begin{aligned}
& a_{1}(t)=\frac{1}{2}\left(a_{1}+e^{i \psi} a_{2}^{\dagger}\right) e^{r}+\frac{1}{2}\left(a_{1}-e^{i \psi} a_{2}^{\dagger}\right) e^{-r} \\
& a_{2}(t)=\frac{1}{2}\left(e^{i \psi} a_{1}^{\dagger}+a_{2}\right) e^{r}+\frac{1}{2}\left(-e^{i \psi} a_{1}^{\dagger}+a_{2}\right) e^{-r}
\end{aligned}
$$

From these evolution equations of $a_{1}(t), a_{2}(t)$ themselves, it might not be obvious to see the squeezing properties. However, when we look at the forms of the quadrature combinations we can easily see the squeezing. For simplicity, here assume the pump phase $\psi=0$ :

$$
\begin{aligned}
& Q_{1}(t)+Q_{2}(t)=\left(Q_{1}+Q_{2}\right) e^{r} \quad(\text { Antisqueezed }) \\
& Q_{1}(t)-Q_{2}(t)=\left(Q_{1}-Q_{2}\right) e^{-r} \quad(\text { Squeezed }) \\
& P_{1}(t)+P_{2}(t)=\left(P_{1}+P_{2}\right) e^{-r} \quad(\text { Squeezed }) \\
& P_{1}(t)-P_{2}(t)=\left(P_{1}-P_{2}\right) e^{r} \quad(\text { Antisqueezed })
\end{aligned}
$$

We can see that the phase sum $P_{1}(t)+P_{2}(t)$ and amplitude difference $Q_{1}(t)-Q_{2}(t)$ are squeezed, and the fact that they commute guarantees that the uncertainty principle is not violated.

Notice that in the above case we choose the pump phase $\psi=0$ for simplicity. If $\psi$ is other values then different quadrature combinations will be squeezed.

Other than directly solving the evolution equations for the operators, we can also use a matrix method to quickly find the nullifiers (operator combinations that are squeezed) and their corresponding squeezing amount. 
Assume $\psi=0$, i.e. $\kappa=\kappa^{*}$, the differential equation sets Eq.(2.68) can be written in matrix forms:

$$
\left(\begin{array}{c}
\dot{a}_{1}(t) \\
a_{2}(t)
\end{array}\right)=\left(\begin{array}{cc}
0 & \kappa \\
\kappa & 0
\end{array}\right)\left(\begin{array}{c}
a_{1}^{\dagger}(t) \\
a_{2}^{\dagger}(t)
\end{array}\right)
$$

And

$$
\left(\begin{array}{c}
{\dot{a_{1}}}^{\dagger}(t) \\
{\dot{a_{2}}}^{\dagger}(t)
\end{array}\right)=\left(\begin{array}{cc}
0 & \kappa \\
\kappa & 0
\end{array}\right)\left(\begin{array}{l}
a_{1}(t) \\
a_{2}(t)
\end{array}\right)
$$

Add and subtract these two, respectively, we get:

$$
\left(\begin{array}{l}
\dot{Q}_{1}(t) \\
\dot{Q}_{2}(t)
\end{array}\right)=\left(\begin{array}{cc}
0 & \kappa \\
\kappa & 0
\end{array}\right)\left(\begin{array}{l}
Q_{1}(t) \\
Q_{2}(t)
\end{array}\right)
$$

And

$$
\left(\begin{array}{c}
\dot{P}_{1}(t) \\
\dot{P}_{2}(t)
\end{array}\right)=\left(\begin{array}{cc}
0 & -\kappa \\
-\kappa & 0
\end{array}\right)\left(\begin{array}{l}
P_{1}(t) \\
P_{2}(t)
\end{array}\right)
$$

For the $Q$ matrix equation 2.76, it has eigenvalues and eigenvectors

Eigenvalue $\lambda_{+}=\kappa$, with Eigenvector $C_{+}(t)=Q_{1}(t)+Q_{2}(t)$

Eigenvalue $\lambda_{-}=-\kappa$, with Eigenvactor $C_{-}(t)=Q_{1}(t)-Q_{2}(t)$

The diagonal form with eigenvalues and eigenvectors is:

$$
\left(\begin{array}{l}
\dot{C}_{+}(t) \\
\dot{C}_{-}(t)
\end{array}\right)=\left(\begin{array}{ll}
\lambda_{+} & 0 \\
0 & \lambda_{-}
\end{array}\right)\left(\begin{array}{l}
C_{+}(t) \\
C_{-}(t)
\end{array}\right)=\left(\begin{array}{cc}
\kappa & 0 \\
0 & -\kappa
\end{array}\right)\left(\begin{array}{l}
C_{+}(t) \\
C_{-}(t)
\end{array}\right)
$$


Since the eigenvectors have:

$$
\begin{aligned}
& C_{+}(t)=C_{+}(0) e^{\kappa t} \\
& C_{-}(t)=C_{-}(0) e^{-\kappa t}
\end{aligned}
$$

So we have:

$$
\begin{aligned}
& Q_{1}(t)+Q_{2}(t)=\left(Q_{1}+Q_{2}\right) e^{\kappa t} \quad(\text { Antisqueezed }) \\
& \left.Q_{1}(t)-Q_{2}(t)=\left(Q_{1}-Q_{2}\right) e^{-\kappa t} \quad \text { (Squeezed }\right)
\end{aligned}
$$

This is the same as the result when we solved the operators one by one using the evolution equations and then combining them to obtain the squeezing and antisqueezing expressions.

Similarly, for the $P$ matrix equation 2.77, it has eigenvalues and eigenvectors:

$$
\begin{aligned}
& \text { Eigenvalue } \lambda_{+}^{\prime}=\kappa \text {, with Eigenvector } C_{+}^{\prime}(t)=P_{1}(t)-P_{2}(t) \\
& \text { Eigenvalue } \lambda_{-}^{\prime}=-\kappa \text {, with Eigenvactor } C_{-}^{\prime}(t)=P_{1}(t)+P_{2}(t)
\end{aligned}
$$

And they give the results which agree with our previous ones:

$$
\begin{aligned}
& \left.P_{1}(t)-P_{2}(t)=\left(P_{1}-P_{2}\right) e^{\kappa t} \quad \text { (Antisqueezed }\right) \\
& \left.P_{1}(t)+P_{2}(t)=\left(P_{1}+P_{2}\right) e^{-\kappa t} \quad \text { (Squeezed }\right)
\end{aligned}
$$

This matrix method is good for quickly finding the squeezing and antisqueezing operator combinations (nullifiers) without solving every mode's evolution equation one by one. This is useful when we have multiple modes and know their $Q$ and $P$ matrix: once we find their eigenvalues and eigenvectors (which can be easily done in Mathematica), the nullifiers are known as well. 


\section{Chapter 3}

\section{One-way Quantum Computing}

\subsection{Introduction}

In the circuit model of quantum computing (QC) [9], physical systems carry quantum information and undergo a sequence of controlled unitary operations to implement logic gates. One-way quantum computing, proposed by Robert Raussendorf and Hans J. Briegel in 2001 [38], is different from the circuit model. In the one-way QC model, the initial quantum information and entanglement are prepared and embedded ahead of time in a "quantum substrate" called a cluster state [50]. The computation is then carried out by mere measurements of single qubits and quantum teleportation. The choice of the measurement basis determines the quantum algorithm. It is called "one-way" because the measurements used to imprint a quantum circuit on the state also destroy the entanglement at the same time, but only that of the measured qubit: unlike, say, a Greenberger-Horne-Zeilinger (GHZ) state, the entanglement in a cluster entangled state is resilient to measurements.

We can see that the cluster state is a very crucial part of one-way QC - it is the 
resource and substrate for the whole calculation and information processing, and the quantum information virtually exists in the cluster state through all the entanglement and structures. In the following sections we introduce the cluster state, and then explain how one-way quantum computing works with cluster state. 


\subsection{Cluster states}

In the following I summarize the main concepts and properties of the qubit cluster state, from the original 2001 cluster state paper by Hans J. Briegel and Robert Raussendorf [50]. I will use the original examples and figures given in that paper in the following subsections.

\subsubsection{Generation and definition}

To generate a qubit cluster state, one can use the quantum Ising model of a spin chain (1D) or a spin lattice (2D or 3D).

Consider an ensemble of qubits located on a $d$-dimensional lattice $(d=1,2,3)$ at sites $a$ and interacting via some short-range interactions described by the Hamiltonian:

$$
H_{\text {int }}=h g(t) \sum_{a, a^{\prime}} f\left(a-a^{\prime}\right) \frac{1+Z^{(a)}}{2} \frac{1-Z^{\left(a^{\prime}\right)}}{2}
$$

where $f\left(a-a^{\prime}\right)$ specifies the interaction range. Here we consider a one-dimensional chain and restrict ourselves to next-neighbor interactions only, i.e. $f\left(a-a^{\prime}\right)=\delta_{a+1, a^{\prime}}$. Then we can show that the interaction 3.1 realizes simultaneous conditional phase gates between all qubits at neighboring sites $a$ and $a^{\prime}$, if all qubits are initially prepared in the state of eigenstate $X:\left(|0\rangle_{a}+|1\rangle_{a}\right) / \sqrt{2}$. (If they are prepared in the state $|0\rangle$ or $|1\rangle$, no entanglement will build up). The unitary transformation is:

$$
U(\varphi)=\exp \left(-i \varphi \sum_{a} \frac{1+Z^{(a)}}{2} \frac{1-Z^{(a+1)}}{2}\right)
$$


where $\varphi=\int d t g(t)$. For the values $\varphi=\pi, 3 \pi, 5 \pi, \ldots$ (the gate $U(\pi)$ is called a controlled-Z $C_{Z}$ gate), the resulting state can be written in the form:

$$
\left|\phi_{N}\right\rangle=\frac{1}{2^{N / 2}} \otimes_{a=1}^{N}\left(|0\rangle_{a} Z^{a+1}+|1\rangle_{a}\right)
$$

with the convention $Z^{(N+1)} \equiv 1$.

This Eq. (3.3) describes the state of a system in which $C_{Z}$ gates were applied between all neighboring qubits, initially in state $\frac{1}{\sqrt{2}}(|0\rangle+|1\rangle)$.

For $N=2$, Eq. (3.3) yields

$$
\begin{aligned}
\left|\phi_{2}\right\rangle & =\frac{1}{2}\left(|0\rangle_{1} Z^{(2)}+|1\rangle_{1}\right)\left(|0\rangle_{2}+|1\rangle_{2}\right) \\
& =\frac{1}{2}\left[|0\rangle_{1}\left(|0\rangle_{2}-|1\rangle_{2}\right)+|1\rangle_{1}\left(|0\rangle_{2}+|1\rangle_{2}\right)\right],
\end{aligned}
$$

which is a maximally entangled state. Implement a local unitary transformation $U$ on qubit 2 ,

$$
U=\left(\begin{array}{cc}
\frac{1}{2} & -\frac{1}{2} \\
\frac{1}{2} & \frac{1}{2}
\end{array}\right)
$$

so that

$$
\begin{aligned}
& \left(\begin{array}{cc}
\frac{1}{2} & -\frac{1}{2} \\
\frac{1}{2} & \frac{1}{2}
\end{array}\right)\left(\begin{array}{c}
1 \\
-1
\end{array}\right)=\left(\begin{array}{l}
1 \\
0
\end{array}\right) \\
& \left(\begin{array}{cc}
\frac{1}{2} & -\frac{1}{2} \\
\frac{1}{2} & \frac{1}{2}
\end{array}\right)\left(\begin{array}{l}
1 \\
1
\end{array}\right)=\left(\begin{array}{l}
0 \\
1
\end{array}\right)
\end{aligned}
$$

After the local unitary transformation (l.u.) on qubit 2, we have the $N=2$ cluster 
state:

$$
\left|\phi_{2}\right\rangle={ }_{l . u .} \frac{1}{\sqrt{2}}\left(|0\rangle_{1}|0\rangle_{2}+|1\rangle_{1}|1\rangle_{2}\right)
$$

Similarly, for $N=3,4$, after local unitary transformations on one or more of the qubits, we have

$$
\begin{aligned}
\left|\phi_{3}\right\rangle & ={ }_{l . u .} \frac{1}{\sqrt{2}}\left(|0\rangle_{1}|0\rangle_{2}|0\rangle_{3}+|1\rangle_{1}|1\rangle_{2}|1\rangle_{3}\right) \\
\left|\phi_{4}\right\rangle= & { }_{l . u .} \frac{1}{\sqrt{2}}\left(|0\rangle_{1}|0\rangle_{2}|0\rangle_{3}|0\rangle_{4}+|0\rangle_{1}|0\rangle_{2}|1\rangle_{3}|1\rangle_{4}\right. \\
& \left.+|1\rangle_{1}|1\rangle_{2}|0\rangle_{3}|0\rangle_{4}-|1\rangle_{1}|1\rangle_{2}|1\rangle_{3}|1\rangle_{4}\right)
\end{aligned}
$$

The above states are one-dimensional cluster state chain with qubit number $N$.

Note that the cluster states and GHZ states only coincide for $N=3$. For $N \geq 3$ no set of local unitary can transform one into the other.

The natural generalization to higher dimensions is a cluster $C$ of qubits, arranged on a lattice. A cluster $C$ has the property that, any two sites $c, c^{\prime} \in C$ are connected (there exists a sequence of neighboring sites between the two qubits), and, any sites $c \in C$ and $a \notin C$ are not connected.

For a higher-dimension cluster state, the general form is:

$$
|\Phi\rangle_{C}=\bigotimes_{c \in C}\left(|0\rangle_{c} \bigotimes_{\gamma \in \Gamma} Z^{(c+\gamma)}+|1\rangle_{c}\right)
$$

with the choice $\Gamma=\{(1,0),(0,1)\}$ for $d=2$ and $\Gamma=\{(1,0,0),(0,1,0),(0,0,1)\}$ for $d=3$. (For the previous one-dimension case, $\Gamma=\{1\}$, referring to the next qubit in the chain.) $Z^{(c+\gamma)} \equiv 1$ when $c+\gamma \notin C$. 
The cluster state 3.11 describes a $d$-dimension system $(d=1,2,3, .$.$) where all$ qubits are initially in the state $|0\rangle+|1\rangle$ (neglect the normalization factor here), and control-Z gates are applied between all the neighboring qubits. The cluster state chain Eq.(3.3) is the $d=1$ case of Eq.(3.11).

The cluster states satisfy the following set of eigenvalue equations:

$$
X^{(a)} \bigotimes_{a^{\prime} \in \mathcal{N}(a)} Z^{\left(a^{\prime}\right)}|\Phi\rangle_{C}= \pm|\Phi\rangle_{C}
$$

where $\mathcal{N}(a)$ specifies the sites of all qubits that interact with the qubit at site $a \in C$ (i.e. $\mathcal{N}(a) \equiv(a+\Gamma) \cup(a-\Gamma))$. The eigenvalues \pm 1 are determined by the distribution of the qubits on the lattice. Because $X^{(a)} \bigotimes_{a^{\prime} \in \mathcal{N}(a)} Z^{\left(a^{\prime}\right)}$ leaves the state unchanged, it is called the stabilizer of the state. Note that for a cluster state, the number of stabilizer is equal to its number of qubits.

\subsubsection{Properties}

There are two important concepts/definitions to the understanding of cluster state: maximal connectedness and persistency of entanglement.

Maximum connectedness: The quantum mechanical state of a set of $n$ qubits is maximally connected if any two qubits can be projected, with certainty, into a pure Bell state by local measurements on a subset of the other qubits.

The one-dimensional chain cluster state $\left|\phi_{N}\right\rangle$ (Eq.(3.3))is maximum connected. For example, for $N=4$ chain $\left|\phi_{4}\right\rangle$ (Eq.(3.10)), we can see that any two qubits can 
be projected into a Bell state by measuring other qubits.

All cluster states are maximally connected. The property of maximal connectedness of $|\Phi\rangle_{C}$ does not depend on the precise shape of the cluster. For higher dimensional cluster state, we can first operate the measurements on the proper qubits to cut it into a one-dimensional chain. Figure 3.1 shows an example of how this can be done.

Consider cluster state $C$ and any two qubits on sites $c^{\prime}, c^{\prime \prime} \in C$. To bring these two qubits into a Bell state, we first select a one-dimensional path $P \subset C$ that connects sites $c^{\prime}$ and $c^{\prime \prime}$ as in Figure 3.1. Then we measure all neighboring qubits surrounding this path in the $Z$ basis. By this procedure, we project the qubits on path $P$ into a state that is, up to local unitary transformations, identical to the state $\left|\phi_{N}\right\rangle$ of the linear chain. This way we can reduce the higher dimensional problem to the one-dimensional problem.

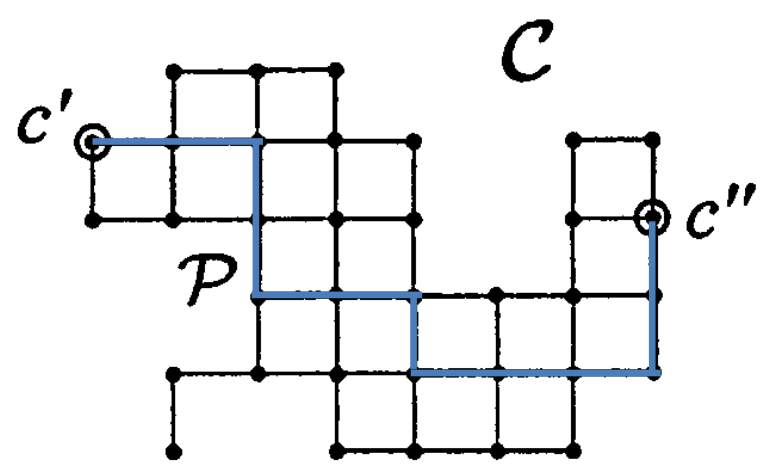

Figure 3.1: Cluster state $C$. Any two qubits $c^{\prime}, c^{\prime \prime}$ of the cluster may be projected into a Bell state by measurements on other qubits of the cluster. From reference [50].

Persistency: The persistency of entanglement $P_{e}$ of an entangled state of $n$ qubits is the minimum number of local measurements such that, for all measurement 
outcomes, the state is completely disentangled (For pure states, a disentangled state means a product state of all $n$ qubits). Obviously, for all $n$-qubit states $0 \leq P_{e} \leq n-1$.

The one-dimensional chain cluster state $\left|\phi_{N}\right\rangle$ 's (Eq.(3.3)) persistency is $P_{e}\left(\left|\phi_{N}\right\rangle\right)=$ $\lfloor N / 2\rfloor$. For example, for $N=4$ chain $\left|\phi_{4}\right\rangle$ (Eq.(3.10)), its persistency $P_{e}\left(\left|\phi_{4}\right\rangle\right)=2$ (one-way of disentangling the state is to measure qubit 1 and 3 in $Z$ basis). In general, however, the exact value of the persistency depends on the shape of a cluster. For large convex clusters, the asymptotic result is $P_{e} / N=1 / 2$ where $N \rightarrow \infty$ is the number of qubits.

\subsubsection{Comparison with other entangled states}

In this subsection, we will compare the entanglement properties and characteristics of three different types of entangled states: the one-dimensional cluster state, the GHZ state, and the $\mathrm{W}$ state.

First let us list the definitions of these three types of states.

\section{$1 \mathrm{D}$ cluster state :}

$$
\left|\phi_{N}\right\rangle=\frac{1}{2^{N / 2}} \otimes_{a=1}^{N}\left(|0\rangle_{a} Z^{a+1}+|1\rangle_{a}\right) .
$$

\section{GHZ state :}

$$
\left|G H Z_{N}\right\rangle=\frac{1}{\sqrt{2}}\left(|0\rangle_{1}|0\rangle_{2} \ldots|0\rangle_{N}+|1\rangle_{1}|1\rangle_{2} \ldots|1\rangle_{N}\right) .
$$

W state :

$$
\left|W_{N}\right\rangle=\frac{1}{\sqrt{N}}\left(|1\rangle_{1}|0\rangle_{2} \ldots|0\rangle_{N}+|0\rangle_{1}|1\rangle_{2}|0\rangle_{3} \ldots|0\rangle_{N}+\ldots+|0\rangle_{1}|0\rangle_{2} \ldots|0\rangle_{N-1}|1\rangle_{N}\right) .
$$


Maximum connectedness: we know that the cluster state $\left|\phi_{N}\right\rangle$ is maximally connected. GHZ state is also maximally connected (just need to measure all qubits but those two that we want to bring into Bell state in $X$ basis). An example for the $N=4$ case is shown below. W state, however, is not maximally connected.

For the $N=4 \mathrm{GHZ}$ state, for example, if we want to project qubit 1 and 2 into a Bell state, we can measure qubit 3 and 4 in the $X$ basis. To see this, write qubit 3 and 4 in the $X$ eigenstate basis $|+\rangle$ and $|-\rangle$ (ignore all the constant factors for simplicity):

$$
\begin{aligned}
\left|G H Z_{4}\right\rangle= & |0000\rangle+|1111\rangle \\
= & |00\rangle_{12}\left(|+\rangle_{3}+|-\rangle_{3}\right)\left(|+\rangle_{4}+|-\rangle_{4}\right)+|11\rangle_{12}\left(|+\rangle_{3}-|-\rangle_{3}\right)\left(|+\rangle_{4}-|-\rangle_{4}\right) \\
= & \left(|00\rangle_{12}+|11\rangle_{12}\right)|++\rangle_{34}+\left(|00\rangle_{12}-|11\rangle_{12}\right)|+-\rangle_{34} \\
& +\left(|00\rangle_{12}-|11\rangle_{12}\right)|-+\rangle_{34}+\left(|00\rangle_{12}+|11\rangle_{12}\right)|--\rangle_{34}
\end{aligned}
$$

when we measure out qubit 3 and 4 on the $X$ basis the state will collapse into a Bell state.

Persistency: we know that the one-dimensional cluster state $\left|\phi_{N}\right\rangle$ has persistency $P_{e}\left(\left|\phi_{N}\right\rangle\right)=\left\lfloor\frac{N}{2}\right\rfloor$. The GHZ state, whose entanglement can be destroyed by just one measurement in the $Z$ basis, clearly has persistency $P_{e}\left(\left|G H Z_{N}\right\rangle\right)=1$. This gives us a quantitative proof that it is more difficult to destroy the entanglement in the state $\left|\phi_{N}\right\rangle$ than that of $\left|G H Z_{N}\right\rangle$ by local operations. The $\mathrm{W}$ state has persistency of 
$P_{e}\left(\left|W_{N}\right\rangle\right)=N-1$, which means the entanglement of $\mathrm{W}$ state is harder to destroy by local measurements than that of $\left|\phi_{N}\right\rangle$. For example, for $\mathrm{W}$ state of $N=3$, $\left|W_{3}\right\rangle=\frac{1}{\sqrt{3}}\left(|1\rangle_{1}|0\rangle_{2}|0\rangle_{3}+|0\rangle_{1}|1\rangle_{2}|0\rangle_{3}+|0\rangle_{1}|0\rangle_{2}|1\rangle_{3}\right)$, when we measure qubit 1 and projected it into $|0\rangle$, then qubit 2 and 3 are still entangled, so we need to measure either one of them again to completely disentangle them. 


\subsection{One-way computing using cluster states}

We have talked about cluster state and its properties, and in this section we will see how to use cluster states to do quantum computing and information processing. Examples and figures are from the original one-way quantum computer paper [38].

Generally speaking, when given a cluster state, we first need to "cut" a certain shape/network of a subset of cluster on which we will implement the measurements. As shown in Figure 3.2. The way to "cut out" the shape we desire is to measure in $Z$ basis all the qubits surrounding it, because by measuring a qubit in the $Z$ basis that qubit will be disconnected from the cluster, which will be left in a state determined by the measurement result. (For example, for a state $|0+\rangle+|1-\rangle$, if we want to leave the second qubit into the $|+\rangle$ state, we need to measure the first qubit and feedforward to the second one.) Then we can implement measurements on the remaining cluster state. In Figure 3.2, the circle qubits are measured in the $Z$ basis so that they are removed, so the rest (shaded area) defines a new cluster "network" (it is related to a building-from-scratch cluster state up to a local unitary transformation depending on the set of $Z$ measurement results).

The measurement basis and measurement order should be determined by the particular computation task, and often the measurement basis of a next qubit will also determined by the results of the proceeding measurements. Usually quantum information is propogated in the horizontal direction by measuring the qubits in a chain and qubits belonging to two parallel chains on vertical connections are used to 


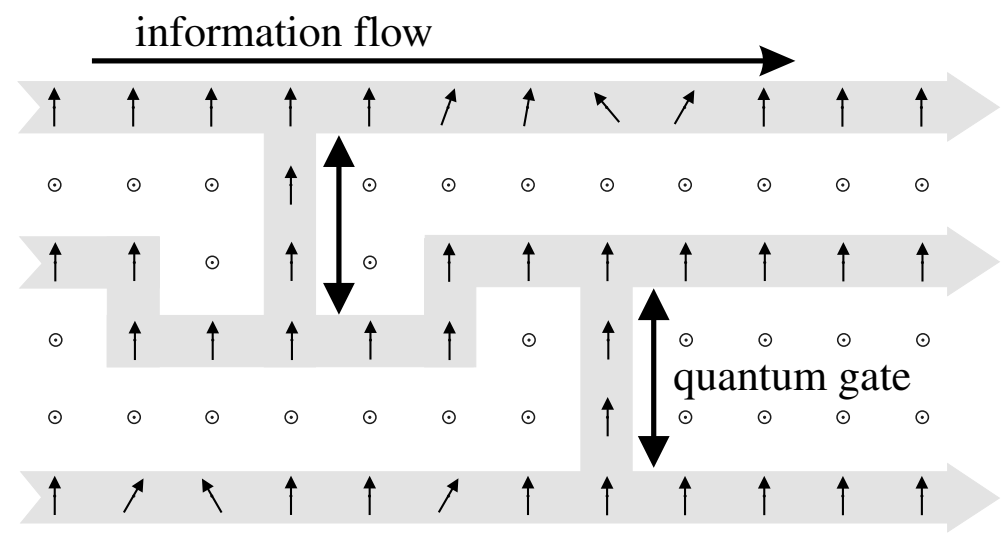

Figure 3.2: Example of quantum computation in a cluster state lattice. The circles symbolize measurements of $Z$ to remove the qubits measured, vertical arrows are measurements of $X$, while tilted arrows refer to measurements in the $x y$ plane. From reference [38].

realize two-bit quantum gates. In the entire process, only one-qubit measurements are required.

In the following I will briefly summarize several basic quantum calculation/processing using the cluster state [38].

\subsubsection{Quantum teleportation in a wire}

Quantum teleportation can be done from one side of the cluster wire to the other site. Consider a chain of the number of qubits 1 to $n$, and locate the qubit we want to teleport at site 1 , and the rest of the qubits in $|+\rangle$ state (eigenstate of $X$ ): $\left|\psi_{i n}\right\rangle_{1} \otimes|+\rangle_{2} \otimes|+\rangle_{3} \ldots \otimes|+\rangle_{n}$

We apply $\mathrm{CZ}$ gates between neighboring qubits (or any other proper entangling interaction) to bring the initial state into a cluster state form. Now we need to measure $X$ at qubits $1,2, \ldots n-1$. The resulting state is $\left|s_{1}\right\rangle_{x, 1} \otimes \ldots \otimes\left|s_{n-1}\right\rangle_{x, n-1} \otimes\left|\psi_{\text {out }}\right\rangle_{n}$. 
The output qubit at site $n$ is related to the input state by a unitary transformation $U_{\Sigma} \in\{1, X, Z, X Z\}$ which depends on the outcomes of the $X$ measurement results at sites 1 to $n-1$. The effect of $U_{\Sigma}$ can be accounted for at the end.

So by simply measuring the $X$ of the first $n-1$ qubit, we can teleport an input state $\left|\psi_{i n}\right\rangle$ to $n$ qubit away (up to some unitary transformation determined by the measurement results which can be easily corrected for).

Take a simple case of two qubits for example. Assume qubit 1 is the input state $\left|\psi_{\text {in }}\right\rangle=a|0\rangle+b|1\rangle$, and qubit 2 is initially at the state $|+\rangle$. After applying the CZ gate, it becomes

$$
\begin{aligned}
& a|0\rangle_{1}\left(|0\rangle_{2}+|1\rangle_{2}\right)+b|1\rangle_{1}\left(|0\rangle_{2}-|1\rangle_{2}\right) \\
= & |+\rangle_{1}\left[(a+b)|0\rangle_{2}+(a-b)|1\rangle_{2}\right]+|-\rangle_{1}\left[(a-b)|0\rangle_{2}+(a+b)|1\rangle_{2}\right]
\end{aligned}
$$

Then we measure $X$ for the first qubit.

When we get the result 1, i.e., qubit 1 is projected into $|+\rangle$ state, then qubit 2 is now $(a+b)|0\rangle_{2}+(a-b)|1\rangle_{2}$, which is equivalent to the gate $U_{\Sigma}=(1,1 / 1,-1)$ applying to the input state $\left|\psi_{i n}\right\rangle$.

When we get the result -1 , i.e., qubit 1 is projected into $|-\rangle$ state, then qubit 2 is now $(a-b)|0\rangle_{2}+(a+b)|1\rangle_{2}$, which is equivalent to the gate $U_{\Sigma}=(1,-1 / 1,1)$ applying to the input state $\left|\psi_{i n}\right\rangle$. 


\subsubsection{An arbitrary rotation $U_{R}$}

An arbitrary rotation can be achieved with a chain of five qubits.

Like the quantum teleportation in a wire case, we locate the state we want to apply the rotate on at site 1 , and the rest 4 qubits all in $|+\rangle$ state, so the initial state is

$$
|\Psi\rangle_{1, \ldots, 5}=\left|\psi_{i n}\right\rangle_{1} \otimes|+\rangle_{2} \otimes|+\rangle_{3} \otimes|+\rangle_{4} \otimes|+\rangle_{5}
$$

Then entangle them with $\mathrm{CZ}$ gates so that they are in the state

$$
\begin{aligned}
\left|\Psi^{\prime}\right\rangle_{1, \ldots, 5}= & \frac{1}{2}\left|\psi_{i n}\right\rangle_{1}|0\rangle_{2}|-\rangle_{3}|0\rangle_{4}|-\rangle_{5}-\frac{1}{2}\left|\psi_{i n}\right\rangle_{1}|0\rangle_{2}|+\rangle_{3}|1\rangle_{4}|+\rangle_{5} \\
& -\frac{1}{2}\left|\psi_{i n}^{*}\right\rangle_{1}|1\rangle_{2}|+\rangle_{3}|0\rangle_{4}|-\rangle_{5}+\frac{1}{2}\left|\psi_{i n}^{*}\right\rangle_{1}|1\rangle_{2}|-\rangle_{3}|1\rangle_{4}|+\rangle_{5},
\end{aligned}
$$

where $\left|\psi_{i n}^{*}\right\rangle=Z\left|\psi_{i n}\right\rangle$.

Then for qubit 1 to 4 conduct a measurement $B_{j}\left(\alpha_{j}\right)=\left\{\frac{|0\rangle_{j}+e^{i \alpha_{j}}|1\rangle_{j}}{\sqrt{2}}, \frac{|0\rangle_{j}-e^{-i \alpha_{j}}|1\rangle_{j}}{\sqrt{2}}\right\}$ (a measurement basis defined by two orthogonal states) whereby the measurement outcomes $s_{j} \in\{0,1\}$ for $j=1, \ldots, 4$ are obtained (here $s_{j}=0$ means the qubit $j$ is projected into the first state of $\left.B_{j}\left(\alpha_{j}\right)\right)$. The resulting state is $\left|s_{1}\right\rangle_{\alpha_{1}, 1} \otimes\left|s_{2}\right\rangle_{\alpha_{2}, 2} \otimes$ $\left|s_{3}\right\rangle_{\alpha_{3}, 3} \otimes\left|s_{4}\right\rangle_{\alpha_{4}, 4} \otimes\left(U\left|\psi_{i n}\right\rangle_{5}\right)$. When we measure qubit 1 in the $X$ basis (choose $\alpha_{1}=0$ ), the unitary transformation $U$ on qubit 5 has the form:

$$
U=X^{s_{2}+s_{4}} Z^{s_{1}+s_{3}} U_{R}\left[(-1)^{s_{1}+1} \alpha_{2},(-1)^{s_{2}} \alpha_{3},(-1)^{s_{1}+s_{3}} \alpha_{4}\right]
$$

Here $U_{R}$ is a rotation operator in Euler representation $U_{R}(\xi, \eta, \zeta)=U_{x}(\xi) U_{z}(\eta) U_{x}(\zeta)$, 
where $U_{x}(\theta)=\exp (-i / 2), U_{z}(\theta)=\exp (-i / 2)$. Thus, to achieve an arbitrary rotation $U_{R}(\xi, \eta, \zeta)$ specified by its Euler angles $\xi, \eta, \zeta$, the measurement basis we need to choose is: for qubit 1 measure in $B_{1}(0)$; for qubit 2 measure in $B_{2}\left((-1)^{s_{1}+1} \xi\right)$; for qubit 3 measure in $B_{3}\left((-1)^{s_{2}} \eta\right)$; for qubit 4 measure in $B_{4}\left((-1)^{s_{1}+s_{3}} \zeta\right)$. In this way the rotation on qubit 5 is $U_{R}^{\prime}=X^{s_{2}+s_{4}} Z^{s_{1}+s_{3}} U_{R}(\xi, \eta, \zeta)$. The extra rotation $X^{s_{2}+s_{4}} Z^{s_{1}+s_{3}}$ can be taken care of and corrected for at the end since the measurement results $s_{1}$ to $s_{4}$ are all known.

\subsubsection{Two-bit operation: CNOT gate}

To apply a CNOT gate between a control qubit and target qubit, we need four qubits arranged as shown in Figure 3.3. Notice this is not a single wire anymore, but a cluster structure that has two wires involved (or can be viewed as a single wire from qubit 1 to 3 and with an additional qubit 4 attached). During the action of the gate, the target qubit is transferred from "target in" to "target out". The qubit 4 is the control qubit.

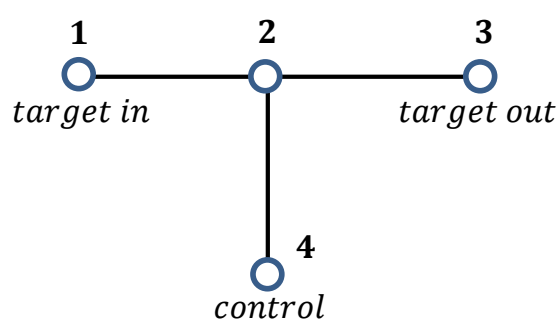

Figure 3.3: Cluster structure for CNOT gate. Qubit 1 is the target qubit and qubit 4 is the control qubit. The CNOT gate result is embedded on qubit 3 "target out". From reference [38].

First prepare the four qubits such that qubit 1 and 4 are in the $Z$ eigenstate, and 
qubit 2 and 3 are in $|+\rangle$ state: $\left|i_{1}\right\rangle_{z, 1}\left|i_{4}\right\rangle_{z, 4}|+\rangle_{2}|+\rangle_{3}$. Then the unitary transformation Eq.(3.2) is applied to generate the cluster state form. Then measure qubit 1 and 2 in the $X$ basis. The resulting quantum state by this procedure is $\left|s_{1}\right\rangle_{x, 1} \otimes\left|s_{2}\right\rangle_{x, 2} \otimes$ $U_{\sum}^{34}\left|i_{4}\right\rangle_{z, 4} \otimes\left|i_{1}+i_{4} \bmod 2\right\rangle_{z, 3}$, where $U_{\sum}^{34}=Z^{(3) s_{1}+1} X^{(3) s_{2}} Z^{(4) s_{1}}$. The input state is thus acted upon by the CNOT and successive $X$ and $Z$ rotations $U_{\Sigma}^{34}$, depending on the measurement results $s_{1}, s_{2}$. From this result we can see that once the $X$ of qubit 1 and 2 have been measured, the value $i_{4}$ of the control qubit (qubit 4) controls whether the target qubit is flipped or not (CNOT). The unwanted rotation $U_{\Sigma}^{34}$ again can be accounted for at the end since its parameters $s_{1}, s_{2}$ are both known.

\subsubsection{Quantum circuits}

The Gottesman-Knill theorem [51] states that any quantum computation that uses only Clifford gates (which leaves the Pauli group globally invariant) can be simulated effectively by a classical computer. Thus, to obtain a speedup of quantum computing over classical computing, at least one non-Clifford gate (such as a $\pi / 4$ rotation $T=$ $\left.e^{i \pi / 8} R_{z}\left(\frac{\pi}{4}\right)=e^{i \pi / 8}\left(\begin{array}{cc}e^{-i \pi / 8} & 0 \\ 0 & e^{i \pi / 8}\end{array}\right)\right)$ is needed.

We know that the CNOT gates plus arbitrary single-qubit operations form a universal gate set; i.e., they are sufficient for universal quantum computing [9], and that an arbitrary single-qubit operator can be decomposed into three rotations, also 
known as three Euler angles (Theorem 4.1 of [9]):

Theorem: $Z-Y$ decomposition for a single qubit operation:

Suppose $U$ is a unitary operation on a single qubit. Then there exist real numbers $\alpha, \beta, \gamma$ and $\delta$ such that

$$
U=e^{i \alpha} R_{z}(\beta) R_{y}(\gamma) R_{z}(\delta)
$$

We can also prove that Hadamard gate (Clifford) plus $\pi / 8$ gate (non-Clifford) can be used to approximate any single qubit unitary operation to arbitrary accuracy as well.

A cluster state can do both CNOT and an arbitrary rotations (actually each require relatively simple cluster structure as shown in the previous subsections), and this ensures that universal quantum gates can be implemented on the cluster state. In order to implement complicated quantum computing tasks, we need to have a sequence of different simple operations.

If we want to apply several consecutive operations with each quantum circuit's output being its following circuit's input, we can either follow the sequence by first entangling the subset of qubits for a particular quantum circuit only and after we obtain its results, continue to entangle the subset of qubits for the following quantum circuit; or we can entangle all the qubits in a single step at the beginning and then perform all measurements afterwards. The second strategy is more compact and is usually how people would do calculation with cluster state. It is also why building a large cluster state is very useful as it prepares beforehand all the necessary entangle- 
ments for the complicated calculations to follow; once we have a cluster state ready, all that is left is to apply the proper local measurements on the right qubits. 


\subsection{From qubits to qumodes: the continuous-variable cluster state}

In the previous section, we have introduced cluster states for qubits. Here, we present a continuous variable encoding of quantum information whose use is justified by the mature experimental methods and techniques of squeezed state generation in quantum optics. In the following I will summarize the generalizations from discretevariable (qubit) to continuous-variable (qumode), and the continuous-variable version of cluster state [52], [53] [54].

\subsubsection{Qubit to qumode generalization}

For qubit, the operator $Z$ 's eigenstates are $|0\rangle$ and $|1\rangle$, and in continuous variable, their corresponding counterpart are eigenstates in the $Q$ quadrature basis: $\left\{|q\rangle_{q}\right\}$. Similarly, the operator $X$ 's eigenstates are $|+\rangle$ and $|-\rangle$ for the qubit, and their counterpart for the continuous variable are the eigenstates in the $P$ quadrature basis

$\left\{|p\rangle_{p}\right\}$. A qumode, which is the counterpart of qubit in continuous-variable, can be represented in either $\left\{|q\rangle_{q}\right\}$ or $\left\{|p\rangle_{p}\right\}$ basis.

The operator $Z$ itself, in the continuous variable representation, becomes:

$$
\mathrm{Z}(\mathrm{t})=\mathrm{e}^{\mathrm{it} \hat{\mathbf{Q}}}
$$

The operator $X$ in the continuous variable representation becomes:

$$
\mathrm{X}(\mathrm{s})=\mathrm{e}^{-\mathrm{is} \hat{\mathbf{P}}}
$$


It is straightforward to see that $|q\rangle$ is an eigenstate of the operator $Z(t)$, with eigenvalue $e^{i t q}, \forall q$, and that $|p\rangle$ is an eigenstate of the operator $X(s)$, with eigenvalue $e^{-i s p}, \forall p$. As mentioned above, the action of the $X$ and $Z$ shift operators is given by,

$$
Z(t)|p\rangle_{p}=|p+t\rangle_{p}
$$

in the phase representation $\left\{|p\rangle_{p}\right\}$, and

$$
X(s)|q\rangle_{q}=|q+s\rangle_{q}
$$

in the amplitude representation $\left\{|q\rangle_{q}\right\}$.

In the following I will remind how to obtain Eqs.(3.21), in an identical manner to quantized position and momentum.

Both representations are related by the quantum Fourier transform:

$$
\begin{aligned}
& |p\rangle_{p}=\frac{1}{\sqrt{2 \pi}} \int_{-\infty}^{\infty} e^{i p q}|q\rangle_{q} \mathrm{~d} q \\
& |q\rangle_{q}=\frac{1}{\sqrt{2 \pi}} \int_{-\infty}^{\infty} e^{-i q p}|p\rangle_{p} \mathrm{~d} p
\end{aligned}
$$


whose unitary operator $\mathrm{F}$ we define as

$$
\begin{aligned}
& F|s\rangle_{q}=|s\rangle_{p} \\
& F^{\dagger}|s\rangle_{p}=|s\rangle_{q}
\end{aligned}
$$

In optics, the Fourier transform operator can also be viewed as a simple optical phase shift, a.k.a. a $\pi / 2$ rotation for a single qumode: $F=R(\pi / 2)$, where the rotation matrix $M_{R}(\theta)$ is defined as:

$$
\left(\begin{array}{l}
\hat{Q} \\
\hat{P}
\end{array}\right) \rightarrow\left(\begin{array}{cc}
\cos \theta & -\sin \theta \\
\sin \theta & \cos \theta
\end{array}\right)\left(\begin{array}{l}
\hat{Q} \\
\hat{P}
\end{array}\right)=M_{R}(\theta)\left(\begin{array}{l}
\hat{Q} \\
\hat{P}
\end{array}\right)
$$

Now let us go back to prove Eqs. (3.21). To show that, we can decompose $|p\rangle_{p}$ into $|q\rangle_{q}$ basis (or vice versa):

$$
\begin{aligned}
Z(t)|p\rangle_{p} & =e^{i t \hat{Q}}|p\rangle_{p} \\
& =\frac{1}{\sqrt{2 \pi}} \int e^{i p q} e^{i t \hat{Q}}|q\rangle_{q} \mathrm{~d} q \\
& =\frac{1}{\sqrt{2 \pi}} \int e^{i p q} e^{i t q}|q\rangle_{q} \mathrm{~d} q \\
& =\frac{1}{\sqrt{2 \pi}} \int e^{i(p+t) q}|q\rangle_{q} \mathrm{~d} q \\
& =|p+t\rangle_{p}
\end{aligned}
$$


Similarly,

$$
\begin{aligned}
X(s)|q\rangle_{q} & =e^{-i s \hat{P}}|q\rangle_{q} \\
& =\frac{1}{\sqrt{2 \pi}} \int e^{-i p q} e^{-i s \hat{P}}|p\rangle_{p} \mathrm{~d} p \\
& =\frac{1}{\sqrt{2 \pi}} \int e^{-i p q} e^{-i s p}|p\rangle_{p} \mathrm{~d} p \\
& =\frac{1}{\sqrt{2 \pi}} \int e^{-i(q+s) p}|p\rangle_{p} \mathrm{~d} p \\
& =|q+s\rangle_{q}
\end{aligned}
$$

Thus, Eqs. 3.21 are proved.

The two-qubit gates controlled-X $C_{X}$ and controlled-Z $C_{Z}$ also have their $\mathrm{CV}$ counterparts, defined as,

$$
C_{X}=e^{-i \hat{Q}_{1} \hat{P}_{2}}
$$

And

$$
C_{Z}=e^{i \hat{Q}_{1} \hat{Q}_{2}}
$$

where qumode 1 is the control qumode and 2 is the target qumode.

The controlled-X operator performs a position-shift defined by the controlled qumode on the target qumode:

$$
C_{X}\left|s_{1}\right\rangle_{q}\left|s_{2}\right\rangle_{q}=\left|s_{1}\right\rangle_{q}\left|s_{1}+s_{2}\right\rangle_{q}
$$


To see this, we write out:

$$
\begin{aligned}
& C_{X}\left|s_{1}\right\rangle_{q}\left|s_{2}\right\rangle_{q} \\
= & e^{-i \hat{Q}_{1} \hat{P}_{2}}\left|s_{1}\right\rangle_{q}\left|s_{2}\right\rangle_{q} \\
= & \left|s_{1}\right\rangle_{q} e^{-i s_{1} \hat{P}_{2}}\left|s_{2}\right\rangle_{q} \\
= & \left|s_{1}\right\rangle_{q} X\left(s_{1}\right)\left|s_{2}\right\rangle_{q} \\
= & \left|s_{1}\right\rangle_{q}\left|s_{1}+s_{2}\right\rangle_{q}
\end{aligned}
$$

Similarly, for the $C_{Z}$ gate, we have:

$$
C_{Z}\left|s_{1}\right\rangle_{q}\left|s_{2}\right\rangle_{p}=\left|s_{1}\right\rangle_{q}\left|s_{1}+s_{2}\right\rangle_{p}
$$

To see this, we write out:

$$
\begin{aligned}
& C_{Z}\left|s_{1}\right\rangle_{q}\left|s_{2}\right\rangle_{p} \\
= & e^{i \hat{Q}_{1} \hat{Q}_{2}}\left|s_{1}\right\rangle_{q}\left|s_{2}\right\rangle_{p} \\
= & \left|s_{1}\right\rangle_{q} e^{i s_{1} \hat{Q}_{2}}\left|s_{2}\right\rangle_{p} \\
= & \left|s_{1}\right\rangle_{q} Z\left(s_{1}\right)\left|s_{2}\right\rangle_{p} \\
= & \left|s_{1}\right\rangle_{q}\left|s_{1}+s_{2}\right\rangle_{p}
\end{aligned}
$$

It is important to know that the vacuum state $|0\rangle$, in the continuous variable representations, has a Gaussian wavefunction centered at 0:

$$
|0\rangle=\frac{1}{\pi^{1 / 4}} \int e^{-s^{2} / 2}|s\rangle_{q} \mathrm{~d} s=\frac{1}{\pi^{1 / 4}} \int e^{-s^{2} / 2}|s\rangle_{p} \mathrm{~d} s
$$




\subsubsection{Gaussian and non-Gaussian operations}

The Gottesman-Knill theorem [51] (any quantum computation that uses only Clifford gates can be simulated effectively by a classical computer) can be formulated in the CV case: any quantum evolution consisting solely of Gaussian operations on Gaussian states may be efficiently simulated on a classical computer [55]. Thus, in order to do universal quantum computer with continuous variable, at least one non-Gaussian operation is needed [52].

Gaussian states are those states whose Wigner function (a quasiprobability distribution over the phase space) has the Gaussian distribution. The operators to preserve the Gaussian property (to transform one Gaussian state into another Gaussian state) are called Gaussian operators.

Some standard single-mode Gaussian operations include [53]:

Rotations: $R(\theta)=e^{\theta\left(\hat{Q}^{2}+\hat{P}^{2}\right) / 2}$. It rotates a state in the phase space counterclockwise by an angle $\theta$, also referred to as phase shifts.

$$
\left(\begin{array}{l}
\hat{Q} \\
\hat{P}
\end{array}\right) \rightarrow\left(\begin{array}{cc}
\cos \theta & -\sin \theta \\
\sin \theta & \cos \theta
\end{array}\right)\left(\begin{array}{l}
\hat{Q} \\
\hat{P}
\end{array}\right)=M_{R}(\theta)\left(\begin{array}{l}
\hat{Q} \\
\hat{P}
\end{array}\right)
$$

Quadrature displacement: As we mentioned before, operator $Z(t)=e^{i t \hat{Q}}$ displaces the momentum quadrature $p$ by an amount of $t: Z(t)|p\rangle_{p}=|p+t\rangle_{p}$. 


$$
\left(\begin{array}{l}
\hat{Q} \\
\hat{P}
\end{array}\right) \rightarrow\left(\begin{array}{l}
\hat{Q} \\
\hat{P}
\end{array}\right)+\left(\begin{array}{l}
0 \\
t
\end{array}\right)
$$

Similarly, operator $X(s)=e^{-i s \hat{P}}$ displaces the position quadrature $q$ by an amount of $s: X(s)|q\rangle_{q}=|q+s\rangle_{q}$.

$$
\left(\begin{array}{l}
\hat{Q} \\
\hat{P}
\end{array}\right) \rightarrow\left(\begin{array}{l}
\hat{Q} \\
\hat{P}
\end{array}\right)+\left(\begin{array}{l}
s \\
0
\end{array}\right)
$$

Squeezing: $S(s)=e^{-i \ln s(\hat{Q} \hat{P}+\hat{P} \hat{Q}) / 2}$ squeezes the position quadrature by a factor of $s$ while stretch the conjugate quadrature by $1 / s$ (notice for the squeezing parameter instead of using $r$ like before, here we use $\ln s$ so that the new squeezed and antisqueezed quadratures will not have the exponential form, but they are essentially the same):

$$
\left(\begin{array}{l}
\hat{Q} \\
\hat{P}
\end{array}\right) \rightarrow\left(\begin{array}{cc}
s & 0 \\
0 & 1 / s
\end{array}\right)\left(\begin{array}{l}
\hat{Q} \\
\hat{P}
\end{array}\right)
$$

Shearing: $D_{2, \hat{Q}}(s)=e^{i s \hat{Q}^{2} / 2}$ shears a state with respect to the $\hat{Q}$ axis by a gradient 
of $s$. It is also referred to as the phase gate:

$$
\left(\begin{array}{l}
\hat{Q} \\
\hat{P}
\end{array}\right) \rightarrow\left(\begin{array}{ll}
1 & 0 \\
s & 1
\end{array}\right)\left(\begin{array}{l}
\hat{Q} \\
\hat{P}
\end{array}\right)
$$

Any arbitrary single-mode Gaussian operation can be decomposed into rotations, quadrature displacement, and either squeezing or shearing operations. The addition of a two-mode Gaussian operator (such as beam splitter operator or $C_{X}, C_{Z}$ operator) allows for arbitrary multi-mode Gaussian operation.

Non-Gaussian operation can have the form of $D_{k, \hat{Q}}=e^{i s \hat{Q}^{k}}$ for $k>2(s \in \mathbf{R})$, such as the cubic phase gate, Kerr effect, etc. (For the preceding Gaussian operations, quadrature displacement operator has $k=1$, shear operator has $k=2$.)

\subsubsection{Continuous-variable cluster state definition}

The concept of qubit cluster state can be generalized into continuous variable regime, with necessary adjustments according to CV definition [54] [56].

For qubit cluster state, each qubit starts in the state $|+\rangle$, which is an +1 eigenstate of $X$ operator. For the continuous variable, each qumode starts with the state $|0\rangle_{p}$, which is the +1 eigenstate of $X(s)$. For the quantum computation, each qubit $Z$ measurement should be replaced by $\hat{Q} \mathrm{CV}$ measurement; each qubit $X$ measurement should be replaced by $\hat{P} \mathrm{CV}$ measurement; the qubit $C_{Z}$ interaction is replaced by the $\mathrm{CV} C_{Z}=e^{i \hat{Q}_{i} \hat{Q}_{j}}$ gate, used to entangle neighboring qumodes $i$ and $j$. 
Now let us take a look at the CV cluster state's stabilizers and nullifiers.

The stabilizers for an unweighted CV cluster state with $n$ qumodes are:

$$
K_{i}(s)=X_{i}(s) \prod_{j \in N(i)} Z_{j}(s), \quad i=1,2, \ldots, n
$$

where $N(i)$ denotes the set of vertices in neighbor of qumode $i, s \in \mathbf{R}$. There are $n$ qumodes in the cluster state graph, so there are $n$ stabilizers as well. Notice the stabilizers, being non-Hermitian, are not observable.

Expand the stabilizers, we obtain:

$$
\begin{aligned}
K_{i}(s) & =X_{i}(s) \prod_{j \in N(i)} Z_{j}(s) \\
& =e^{-i s \hat{P}_{i}} e^{i s \hat{Q}_{j 1}} e^{i s \hat{Q}_{j 1} \ldots e^{i s \hat{Q}_{j m}}} \\
& =e^{-i s\left(\hat{P}_{i}-\hat{Q}_{j 1}-\hat{Q}_{j 2}-\ldots-\hat{Q}_{j n}\right)}
\end{aligned}
$$

where $j_{1}, j_{2}, \ldots j_{m}$ are the neighbors of qumode $i$.

The nullifiers $N_{i}$, defined as $N_{i}|\phi\rangle=0$, form a nullifier space:

$$
N_{i}=\hat{P}_{i}-\sum_{j \in N(i)} \hat{Q}_{j}, \quad i=1,2, \ldots, n
$$

The nullifiers are Hermitian so they are observable. In fact, measuring the particular set of nullifiers constitutes a good experimental test to verify a cluster state, as we will see later.

Any linear combination $N=\sum_{i} c_{i} N_{i}$ satisfies $N|\phi\rangle=0$ as well. Also, $\left[N_{i}, N_{j}\right]=0$ for all $(i, j)$.

Notice the above definitions for stabilizers and nullifiers are based on unweighted 
CV cluster state graph where all the $C_{Z}$ entangling interactions between the modes have the same strength (or equivalently, graphs whose edge weights are all +1 ).

When the weights are unequal, the nullifiers have the form:

$$
N_{i}^{\prime}=\hat{P}_{i}-\sum_{j \in N(i)} A_{i j} \hat{Q}_{j}, \quad i=1,2, \ldots, n
$$

where $A_{i j}$ is the weight between qumodes $i$ and $j$, also known as the cluster state graph $\mathbf{A}$ adjacency matrix defining the cluster state graph shapes and weights.

For an Einstein-Podolsky-Rosen (EPR) state, the cluster state $\mathbf{A}$ graph is shown as follows, and $A_{12}=A_{21}=1$.

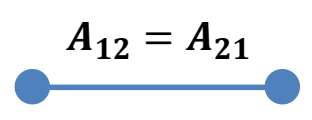

Figure 3.4: Graph A for EPR state (neglect any self loops).

So the nullifiers for the EPR state are,

$$
\begin{aligned}
& \hat{P}_{1}-\hat{Q}_{2} \\
& \hat{P}_{2}-\hat{Q}_{1}
\end{aligned}
$$

When applying a $\pi / 2$ local phase rotation to mode 1 , the above nullifiers become $\hat{P}_{1}+\hat{P}_{2}$ and $\hat{Q}_{1}-\hat{Q}_{2}$. Experimentally, these are what we measure to verify the cluster state structure: we measure the nullifiers using homodyne detection, and the noise of those will be below that of the shot noise (vacuum noise), and this is called "squeezing", as introduced in the previous chapter. 


\section{Chapter 4}

\section{The Optical Parametric Oscillator}

The Optical Parametric Oscillator, also known as OPO, is an important element in quantum optics and photonics in general. In this section I will talk about the OPO, its building parts, basic concepts and parameters, and nonlinear crystals. 


\subsection{Constituents}

An OPO has two essential parts: the optical cavity and the nonlinear medium inside the cavity. An example of a typical OPO is shown in Figure 4.1.

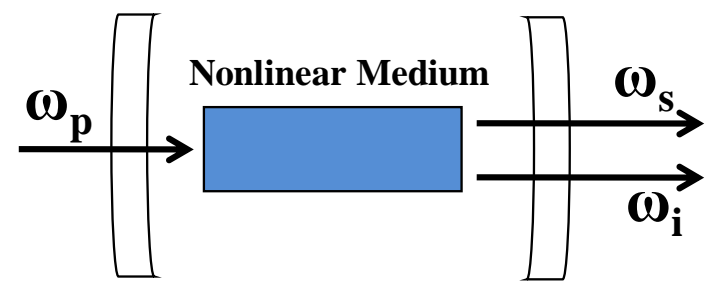

Figure 4.1: An example of a typical OPO with an optical cavity and the nonlinear medium. When the nonlinear medium is a second-order nonlinear crystal, a pump photon entering with frequency $\omega_{p}$ will down convert and generate two photons (called signal and idler) with frequencies $\omega_{s}$ and $\omega_{i}$ respectively, satisfying $\omega_{p}=\omega_{i}+\omega_{s}$.

In the following sections, I will talk about the optical cavity and the nonlinear medium respectively. 


\subsection{Optical cavity}

The optical cavity, also known as optical resonator, can have two mirrors, or more than two mirrors. In a ring cavity, the optical beam propagates in one direction and during one round trip, the path does not overlap. In our experiment, we used a fourmirror ring cavity for both the OPO and the filter cavity, as shown in Figure 4.2(c) (details in next chapter).

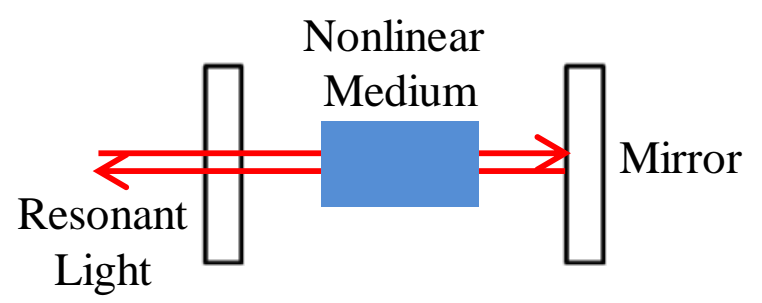

(a) OPO with two mirrors

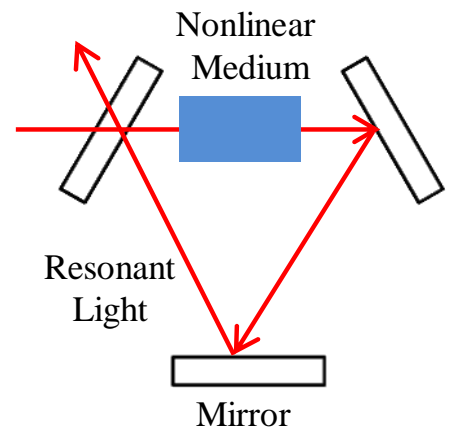

(b) OPO with three mirrors

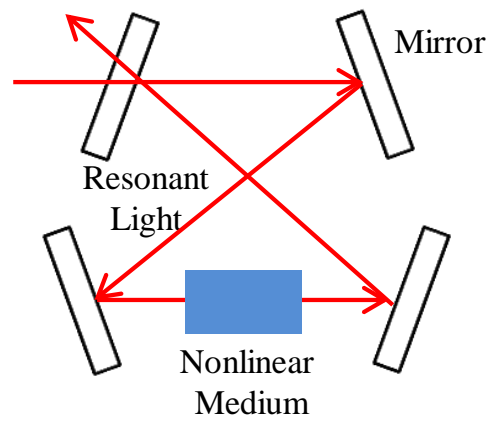

(c) OPO with four mirrors

Figure 4.2: OPOs with different optical cavity types. Figure 4.2(a) OPO cavity has two mirrors, and the path of one round trip "folds up" and the two opposite prorogating directions overlap (in the drawing there is some space between them just to show the distinct directions); Figure 4.2(b) and Figure 4.2(c) have more than 2 mirrors, forming a ring cavity. In a ring cavity, the optical beam propagates in one direction and during one round trip, the optical path is a single path.

In an OPO, usually there are three fields: the pump field, the signal field and 
the idler field (in other cases like four-wave mixing the fields are different but we do not worry about those cases here). The pump photon, after sending through the nonlinear medium (usually a nonlinear crystal), will convert into two new photons (signal and idler) whose frequencies add up to the frequency of the pump when the phase matching conditions (details in the next section) are satisfied, and this process is called "down conversion".

For an OPO usually there are two types of resonance for the signal and idler fields: singly resonance and doubly resonance. Singly resonant OPO is the type of OPO that either signal or idler is resonant (only one of them, but not both) in the optical cavity; while doubly resonant OPO has both the signal and the idler resonant in its optical cavity.

There are a few basic and important concepts and parameters about an optical cavity, and I will list them in this section.

\subsubsection{Free spectral range}

Free Spectral Range (FSR) is one of the most important parameters of an OPO.

For an optical light to be resonant inside an optical cavity, the beams coming from different round trips need to constructively interfere, which requires their phase differences of different round trips to be an integer multiple of $2 \pi$. The phase difference for one round trip is $e^{i k L}=e^{i 2 \pi f L / c}$. So it requires: 


$$
\begin{aligned}
& \Delta \phi=\frac{2 \pi f L}{c}=n 2 \pi \\
& \rightarrow f=n \frac{c}{L}
\end{aligned}
$$

where $c$ is the speed of light and $L$ is the optical path of a round trip inside OPO.

Thus, The frequency of the light resonant in the OPO has to satisfy the relation:

$$
f_{\text {res }}=n F S R, \quad(\mathrm{n} \text { is an integer })
$$

The FSR is defined as:

$$
\mathbf{F S R}=\frac{c}{L}
$$

Eq.(4.2) is a simple yet important relation, from which it is easy to see that all the modes that are able to be resonant inside an OPO are equally spaced, separated by a fixed space (FSR) in the frequency domain. The resonant modes form a "comb" in the frequency regime, as shown in Figure 4.3, so we call them "Optical Frequency Comb" (OFC).

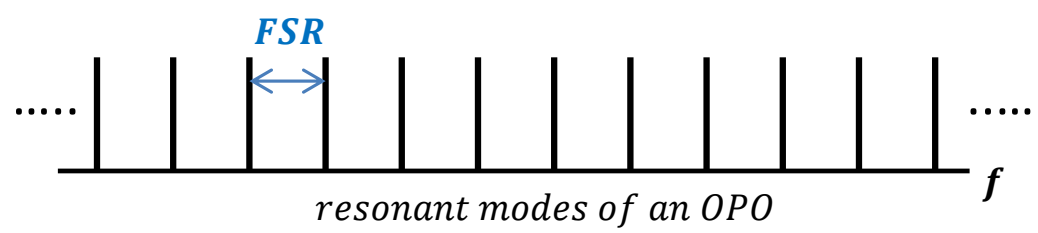

Figure 4.3: Optical Frequency Comb of an OPO. The resonant modes are equally spaced, and the space between the neighboring modes is free spectral range. (Here we just use vertical lines to represent the resonant modes for simplicity.) 


\subsubsection{Linewidth and Finesse}

The transmission ratio of an optical cavity (assuming no other loss) depends on the cavity mirrors' transmission and reflection coefficients. For a two-mirror optical cavity's reflection and transmission coefficients, we can derive them as follows.

Figure 4.4 shows when a light beam hits the input mirror of an optical cavity, the reflection and transmission beams of the cavity. In the figure the incident and reflection angles are exaggerated in order to show the individual beams from each round trip, and in reality they should be overlapped.

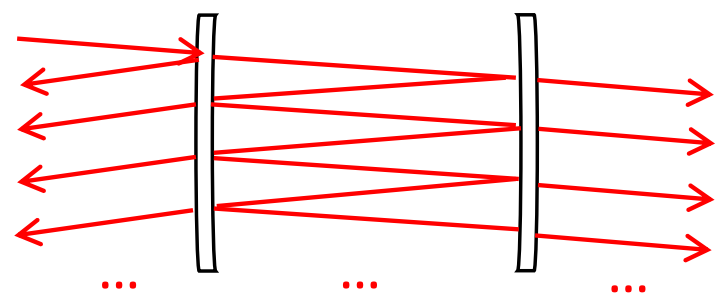

Figure 4.4: The reflection and transmission of an optical cavity when a light beam hits the input mirror.

Adding the amplitudes of all the corresponding electromagnetic fields for the reflection and transmission beams respectively will give us the total reflection and transmission fields.

The reflection field is obtained by adding all the reflection beams (on the left side 
in Figure 4.4) together:

$$
\begin{aligned}
E_{r} & =E_{1 r}+E_{2 r}+E_{3 r}+\ldots \\
& =-r_{1} E_{0}+t_{1}^{2} r_{2} e^{i \delta} E_{0}+t_{1}^{2} r_{2} e^{i \delta} r_{1} r_{2} e^{i \delta}+\ldots \\
& =-r_{1} E_{0}+t_{1}^{2} r_{2} e^{i \delta} \frac{1}{1-r_{1} r_{2} e^{i \delta}} E_{0} \\
& =\frac{r_{2} e^{i \delta}-r_{1}}{1-r_{1} r_{2} e^{i \delta}} E_{0}
\end{aligned}
$$

The transmission field is obtained by adding all the transmission beams (on the right side in Figure 4.4) together:

$$
\begin{aligned}
E_{t} & =E_{1 t}+E_{2 t}+\ldots \\
& =t_{1} t_{2} e^{i \delta / 2} E_{0}+t_{1} t_{2} e^{i \delta / 2} r_{1} r_{2} e^{i \delta} E_{0}+\ldots \\
& =\frac{t_{1} t_{2} e^{i \delta / 2}}{1-r_{1} r_{2} e^{i \delta}} E_{0}
\end{aligned}
$$

where $r_{1}, r_{2}$ are the reflection coefficients of the front and back mirrors of the optical cavity, and $t_{1}, t_{2}$ are the transmission coefficients of the front and back mirrors. $\delta$ is the optical phase for the round trip of the cavity, $\delta=2 \pi f L / c$, where $L$ is the optical path of a round trip of the optical cavity.

The transmittance is:

$$
\begin{aligned}
T & =\left|\frac{E_{t}}{E_{0}}\right|^{2} \\
& =\frac{t_{1}^{2} t_{2}^{2}}{1+r_{1}{ }^{2} r_{2}^{2}-2 r_{1} r_{2} \cos \delta}
\end{aligned}
$$


Notice when $r_{1}=r_{2}$ we have $T_{\max }=1$, otherwise $T_{\max }<1$.

Assume the two mirrors have equal reflectivity: $r_{1}{ }^{2}=r_{2}{ }^{2}=R$ and $t_{1}{ }^{2}=t_{2}{ }^{2}=$ $T=1-R$, then the transmittance becomes:

$$
\begin{aligned}
T & =\frac{(1-R)^{2}}{1+R^{2}-2 R \cos \delta} \\
& =\frac{1}{1+\frac{4 R}{(1-R)^{2}} \sin ^{2}(\delta / 2)}
\end{aligned}
$$

where the triangle relation $1-\cos \theta=2 \sin ^{2}(\theta / 2)$ is used.

It is straightforward to see from Eq.(4.7) that when $\delta=0, T_{\max }=1$. And the period of the function is when $\delta$ change by $2 \pi$, corresponding to one FSR.

Figure 4.5 is a plot of Eq.(4.7) when $R=0.65$.

The linewidth of a cavity is the full-width half-maximum (FWHM) of a peak. To find out the FWHM, let $T=T_{\max } / 2=1 / 2$, and solve for $\delta$ we obtain $\delta=$ $2 \arcsin \sqrt{\frac{(1-R)^{2}}{4 R}}$. This is the phase at which the transmittance $T$ is half the maximum. For full-width, it is double this value, so we have:

$$
\delta_{F W H M}=4 \arcsin \sqrt{\frac{(1-R)^{2}}{4 R}}
$$




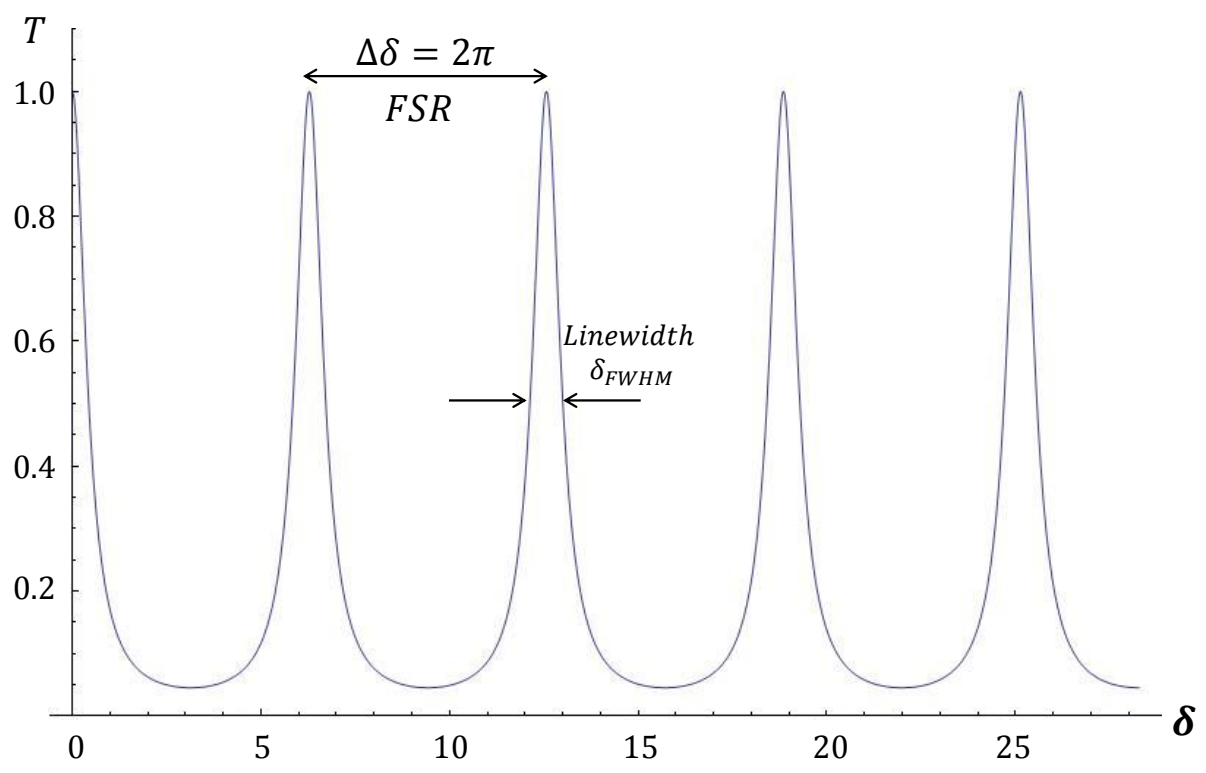

Figure 4.5: Plot of OPO transmittance $T$ as a function of round trip optical phase $\delta$. One FSR corresponds to a change of $2 \pi$ in $\delta$, and the linewidth is defined as the full-width at half-maximum of a peak. Plot at $r_{1}^{2}=r_{2}^{2}=R=0.65$.

Important note: for the finesse to be validly defined, it requires the argument in the arcsin function to be no bigger than $1: \frac{(1-R)^{2}}{4 R} \leq 1$, (which is also required to be able to set $T=1 / 2$ in the first place, otherwise $T$ can never reach $1 / 2$ ). By solving this we obtain that $3-2 \sqrt{2} \leq R \leq 3+2 \sqrt{2}$, i.e. $0.172 \leq R \leq 5.828$. Combine this with the reflectance value range $(0,1)$, the range of $R$ for a valid definition of finesse is $0.172 \leq R<1$. This is known as "Good Cavity Limit". As shown in Figure 4.6, the grey trace has $R=0.16$ and no finesse can be defined.

The finesse is defined as the ratio of the FSR over the FWHM (see Figuire 4.3):

$$
\begin{aligned}
\text { Finesse } & =\frac{\Delta \delta}{\delta_{F W H M}} \\
& =\frac{2 \pi}{4 \arcsin \sqrt{\frac{(1-R)^{2}}{4 R}}}
\end{aligned}
$$


So we have:

$$
\text { Finesse }=\frac{\pi}{2 \arcsin \sqrt{\frac{(1-R)^{2}}{4 R}}}
$$

From the Finesse expression 4.10 we can see that a cavity's finesse is only dependent on its mirrors reflections (given there is no other losses other than the mirror transmission): the bigger the mirrors' reflectance $R$, the bigger the finesse, thus the smaller the linewidth in $\delta$. Figure 4.6 compares two different cavities' finesses and linewidths.

Usually when we talk about OPO's linewidth, we refer to the frequency domain. From Eq.(4.8) and use the round trip phase definition $\delta=2 \pi f L / c$, we have:

$$
\begin{aligned}
f_{F W H M} & =\frac{\delta_{F W H M}}{2 \pi} \frac{c}{L} \\
& =\frac{2 \arcsin \sqrt{\frac{(1-R)^{2}}{4 R}}}{\pi}\left(\frac{c}{L}\right)
\end{aligned}
$$

From Eq.(4.11) we can see that for a cavity with fixed length and fixed crystal and crystal temperature (thus fixed round trip optical path and fixed FSR in frequency), the bigger the $R$, the bigger the finesses, thus the smaller the linewidth in frequency.

Eq.(4.11) shows the interpret of finesse in the frequency domain:

$$
\text { Finesse }=\frac{F S R}{f_{F W H M}}
$$




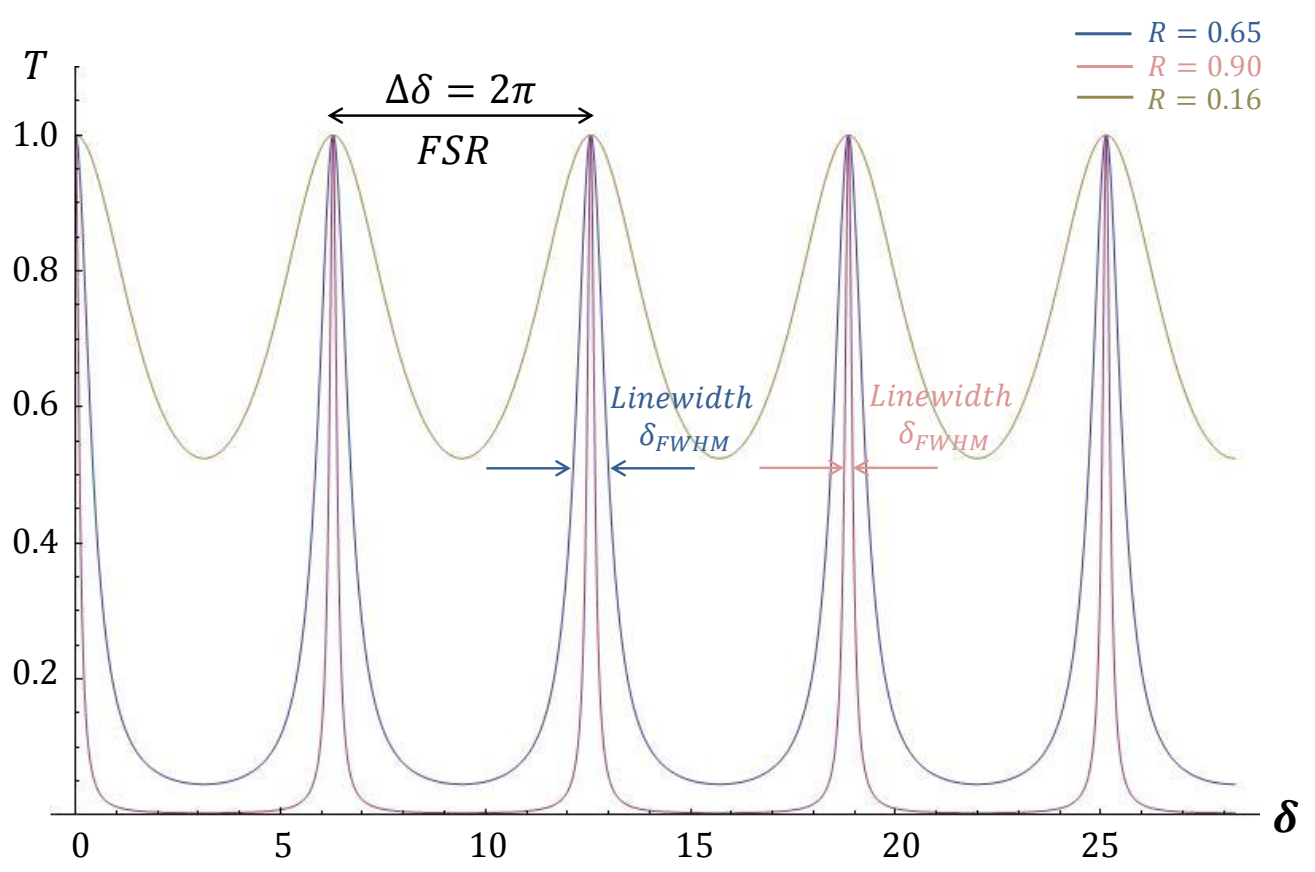

Figure 4.6: OPO transmittance $T$ as a function of round trip optical phase $\delta$ for two different Finesses associated with two different $R \mathrm{~s}$. The blue trace has $R=0.65$ and the red trace has $R=0.9$, notice the grey trace $R=0.16$ has no definition for its finesse (or we could interpret this as its finesse is too small) because its transmittance never drops down to half-maximum: to define finesse, it requires $R \geq 0.172$ (details see the text). Comparing the blue and red traces, we can see that the bigger $R$ results in bigger finesse and smaller linewidth in $\delta$.

For simplicity, sometimes the original finesse form Eq.(4.10) can be approximated using the relation $\frac{1}{\arcsin \sqrt{\frac{(1-R)^{2}}{4 R}}} \approx \sqrt{\frac{4 R}{(1-R)^{2}}}$ when the argument is not too small (i.e. $R$ not too big). Using this approximation we get:

$$
\text { Finesses } \approx \frac{\pi \sqrt{R}}{1-R}
$$

A plot comparing the approximated expression of finesses Eq.(4.13) vs. the original definition Eq.(4.10) is shown in Figure 4.7:

When the two mirrors of the cavity do not have the same reflectivity, i.e. in 


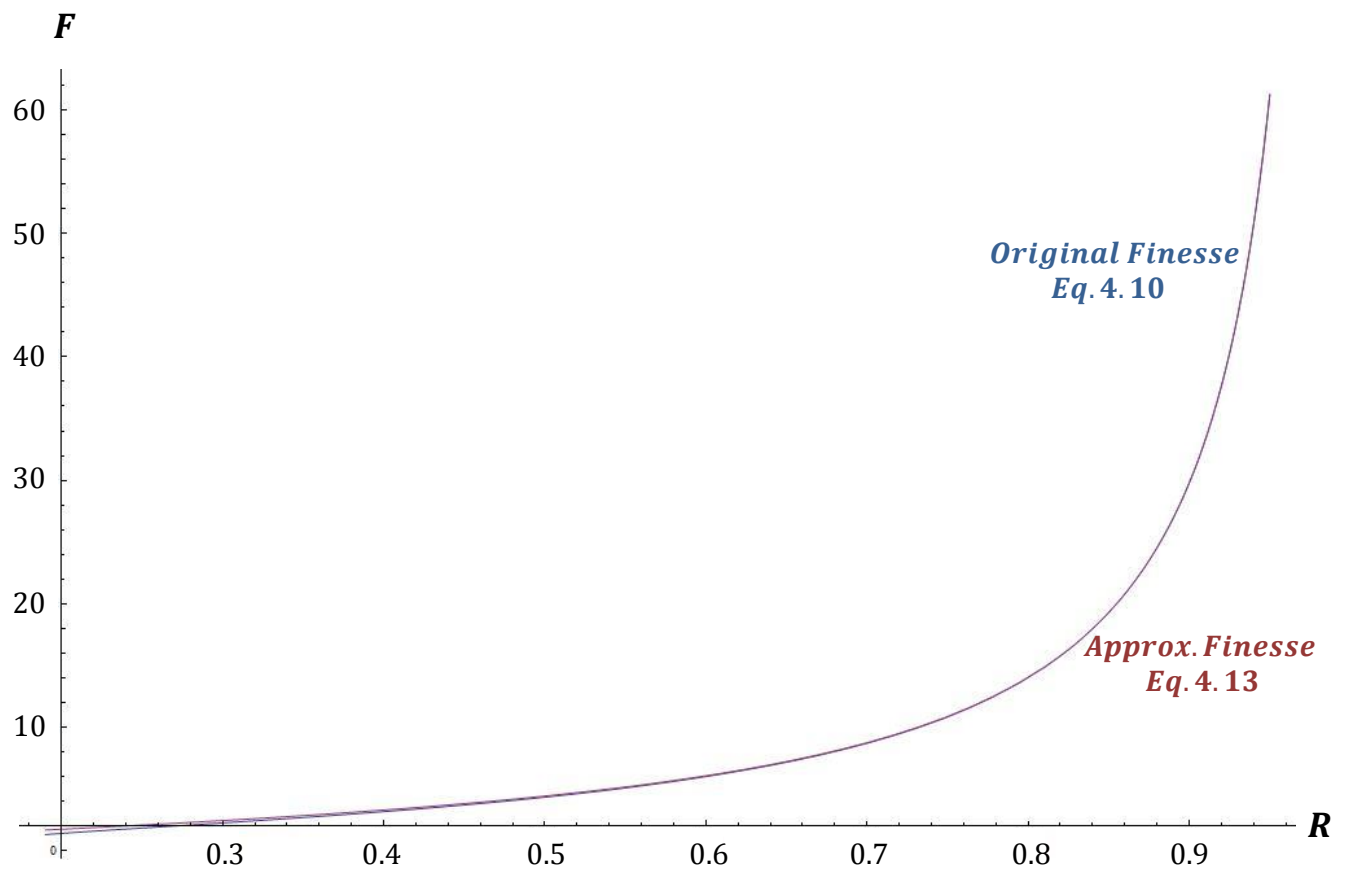

Figure 4.7: Comparison of the original finesse definition (Eq.(4.10)) with the approximated finesse definition (Eq.(4.13)). From the plot we can see that the agreement is fairly well between the original definition and approximated definition for a wide range of $R$. Plot range $R \in[0.19,0.95]$.

Eq.(4.6), $r_{1} \neq r_{2}$, and $r_{1}^{2}=R_{1}, r_{2}^{2}=R_{2}, t_{1}^{2}=1-R_{1}, t_{2}^{2}=1-R_{2}$. Follow the similar derivations as above, we have:

$$
T=\frac{\left(1-R_{1}\right)\left(1-R_{2}\right)}{\left(1-\sqrt{R_{1} R_{2}}\right)^{2}+4 \sqrt{R_{1} R_{2}} \sin ^{2} \delta / 2}
$$

Let $T=T_{\max } / 2=\frac{1}{2} \frac{\left(1-R_{1}\right)\left(1-R_{2}\right)}{\left(1-\sqrt{R_{1} R_{2}}\right)^{2}}$, and solve for $\delta$, we get the $\delta$ at which the transmittance $T$ is half of the maximum. Double that gives the FWHM of $\delta$ :

$$
\delta_{F W H M}=4 \arcsin \frac{1-\sqrt{R_{1} R_{2}}}{2\left(R_{1} R_{2}\right)^{1 / 4}}
$$


Thus, the finesse for the cavity with two different mirrors is:

$$
\text { Finesse }=\frac{\pi}{2 \arcsin \left[\frac{1-\sqrt{R_{1} R_{2}}}{2\left(R_{1} R_{2}\right)^{1 / 4}}\right]}
$$

Similarly, using the approximation $\frac{1}{\arcsin \frac{1-\sqrt{R_{1} R_{2}}}{2\left(R_{1} R_{2}\right)^{1 / 4}}} \approx \frac{2\left(R_{1} R_{2}\right)^{1 / 4}}{1-\sqrt{R_{1} R_{2}}}$ when the argument is not too small (i.e. $R_{1}$ and $R_{2}$ not too big), we get the approximation Finesse for cavity with two different mirrors:

$$
\text { Finesse } \approx \frac{\pi\left(R_{1} R_{2}\right)^{1 / 4}}{1-\sqrt{R_{1} R_{2}}}
$$

It is easy to check that when $r_{1}=r_{2}$, Eq.(4.16) agrees with Eq.(4.10), and that Eq.(4.17) agrees with Eq.(4.13).

Also, when we have one-sided cavity (cavity with one output mirror), we can just use Eq.(4.16) (or 4.17 for approximation) and plug in $R_{1}=1$ and $R_{2}=R$ (or vice versa) to get:

$$
\text { Finesse }_{\text {one-sided cavity }}=\frac{\pi}{2 \arcsin \left[\frac{1-\sqrt{R}}{2(R)^{1 / 4}}\right]}
$$

Compare the finesses of one-sided cavity with two-sided cavity of the same mirror reflectivity (i.e. cavity with one $R<1$ mirror and cavity v.s. two mirrors both have the same $R<1$ ), we have the following plot shown in Figure 4.8: 


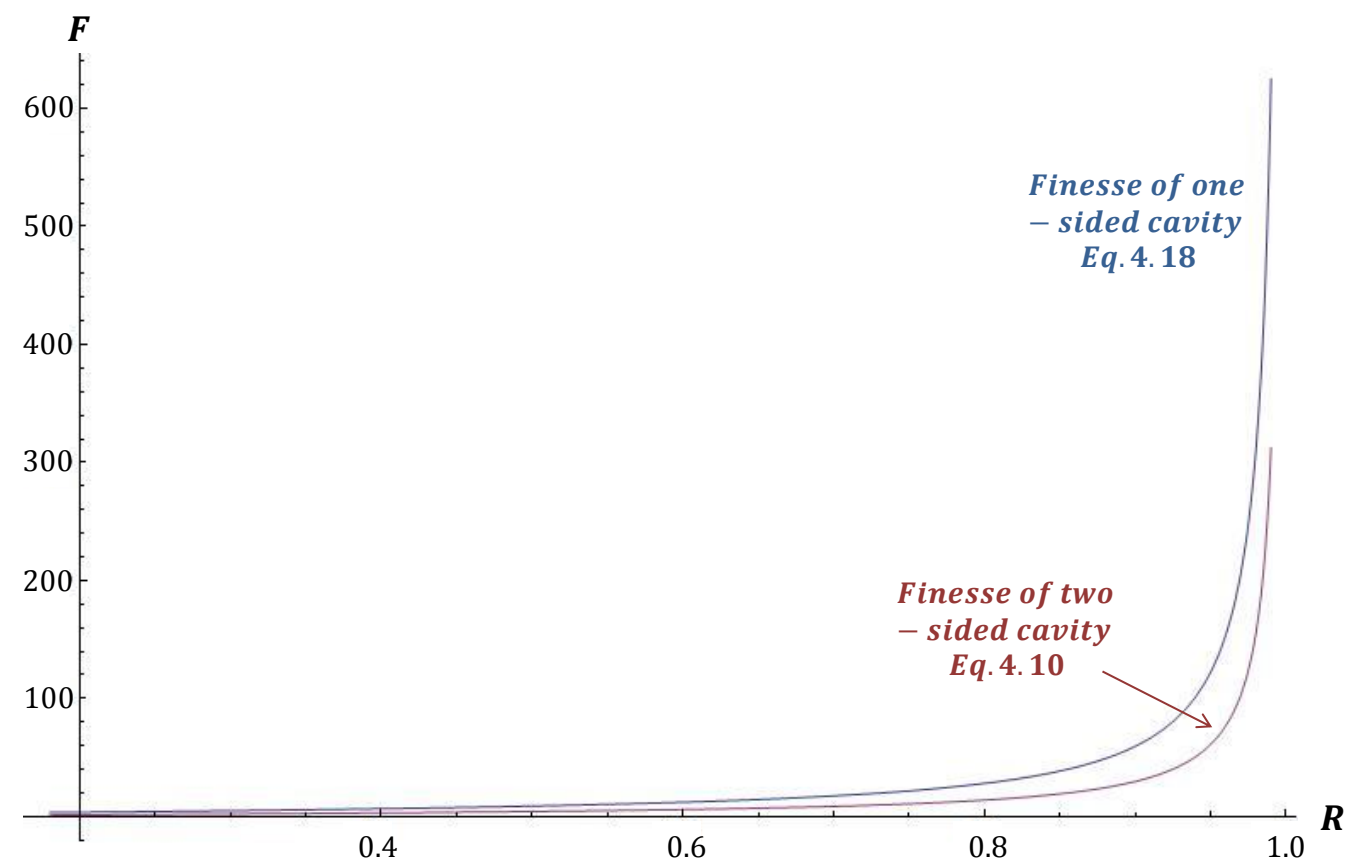

Figure 4.8: Comparison of the one-sided cavity finesse (Eq.(4.18)) with the twosided cavity finesse definition (Eq.(4.10)), for the same mirror reflectivity value $R$. Plot range $R \in[0.18,0.99]$. We can see that the one-sided cavity's finesse is always bigger than that of two-sided cavity (approach about twice as much at large $R$ value $(R \geq 0.8))$.

\subsubsection{Intracavity loss}

Intracavity loss is another important parameter of an OPO. It characterize how lossy the OPO is. The intracavity loss could be due to the reflection of a non-perfect crystal surface, some defects inside the crystal, any scattering dirt inside the cavity, or simply a not-so-clean mirror surface, etc.

To measure the intracavity loss of a ring cavity, we can use the following settings in Figure 4.9. As shown in the figure, we put a photodetector at the reflection part of the cavity while we scan the OPO cavity.

As the OPO cavity being scanned, we should get an oscillating reflection signal 


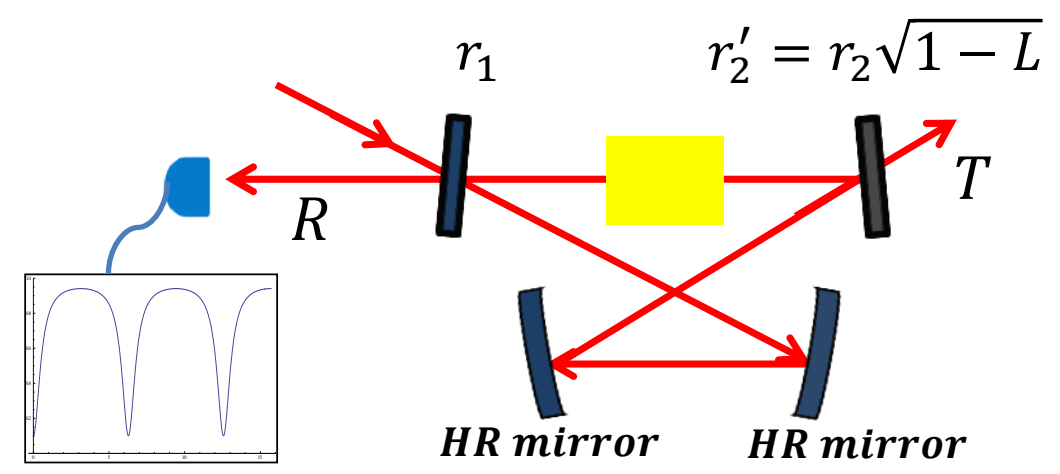

Figure 4.9: The setting to measure the OPO intracavity loss. Measuring the reflection signal from the OPO can determine the intracavity loss. Here we assume a 4-mirror ring cavity with two mirrors on the bottom having high-reflective coatings such that their reflection is $100 \%$. The effective reflection coefficient of the second mirror $r_{2}^{\prime}$ is defined so as to include the effect of the intracavity loss $L$ (see text for details).

as the scan goes through the resonant and non-resonant phases. The signal for the reflection light from the OPO should look like Figure 4.10. Define the height of signal when the OPO is resonant as $a$ and the height when off-resonant as $b$ and define the parameter $k$ as $k \equiv \frac{a}{b}$.

To calculate the intracavity loss $L$, we imbed its effect in the second mirror, i.e. we treat the intracavity loss as part of the second mirror's loss. To realize this, we define a new reflective coefficient $r_{2}^{\prime} \equiv r_{2} \sqrt{1-L}$ that has both the effects of the intracavity loss and the original mirror's loss $r_{2}$. We can see that when there is no intracavity loss $L=0, r_{2}^{\prime}=r_{2}$, so the system is the same as the original system; when there is intracavity loss $0<L<1, r_{2}^{\prime}<r_{2}$, the new effective reflective coefficient $r_{2}^{\prime}$ is smaller than $r_{2}$, indicating more loss than the original mirror (resulting from the intracavity loss).

As shown in Eq. (4.4), a two-sided cavity with mirror reflection coefficient $r_{1}$ and 


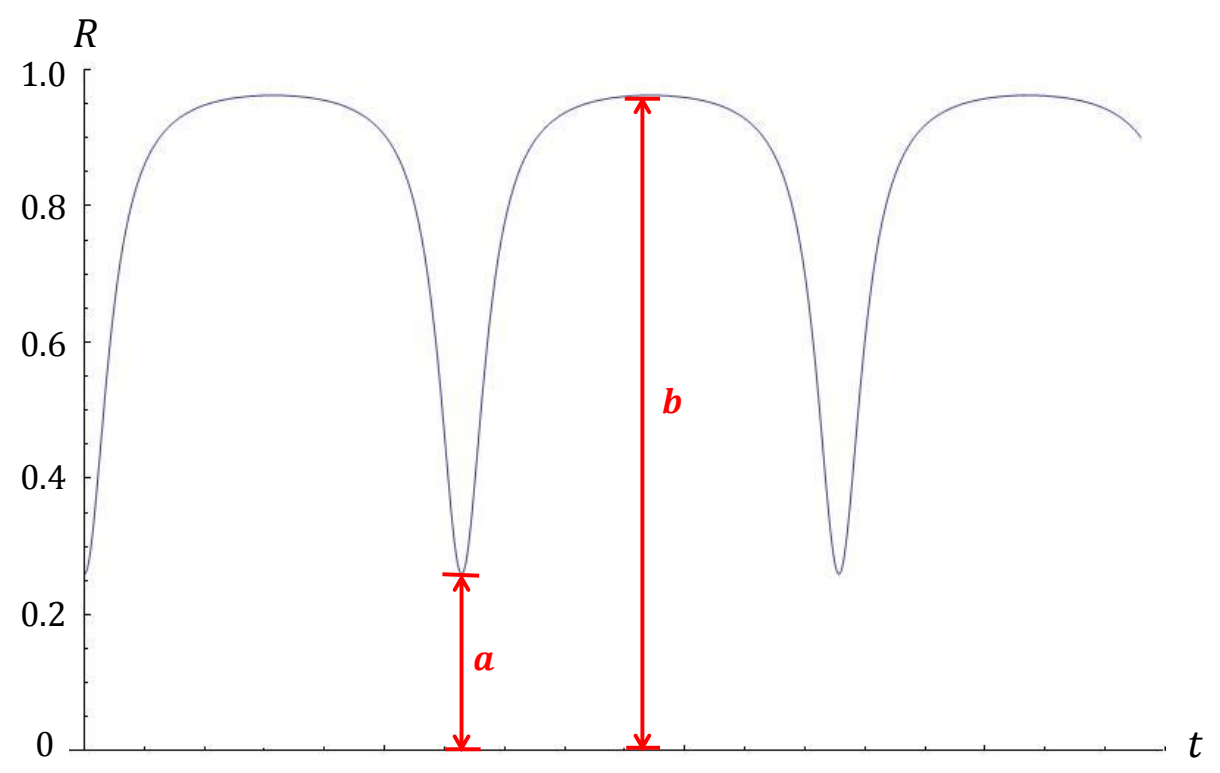

Figure 4.10: A typical reflection signal for intracavity loss measurement. The resonant point (where the reflection signal is the smallest) has height $a$ and the off-resonant point (where the reflection is the biggest) has height $b$. Define the parameter $k$ as $k \equiv \frac{a}{b}$ (see text for details). (In the actual measurement, the whole plot will stretch and multiply the incident power $P_{0}$ but their ratio $k$ is the same, so here we just assume $P_{0}=1$ ). In this example plot $R_{1}=r_{1}^{2}=0.8, R_{2}=r_{2}^{2}=0.5$.

$r_{2}^{\prime}$ has the reflection ratio in $E$ field:

$$
\frac{E_{r}}{E_{0}}=\frac{r_{2}^{\prime} e^{i \delta}-r_{1}}{1-r_{1} r_{2}^{\prime} e^{i \delta}}
$$

The reflection ratio in power is:

$$
\begin{aligned}
R & =\left(\frac{E_{r}}{E_{0}}\right)^{2} \\
& =\left(\frac{r_{2}^{\prime} e^{i \delta}-r_{1}}{1-r_{1} r_{2}^{\prime} e^{i \delta}}\right)^{2} \\
& =\frac{r_{1}^{2}+r_{2}^{\prime 2}-2 r_{1} r_{2}^{\prime} \cos \delta}{1+r_{1}^{2} r_{2}^{\prime 2}-2 r_{1} r_{2}^{\prime} \cos \delta}
\end{aligned}
$$

When the system is on resonant, $\delta=0$; when off-resonant, $\delta=\pi$. (Which is straight- 
forward to see from Eq.(4.6) that when $\delta=0, T=T_{\max }$, and when $\delta=\pi, T=T_{\min }$.) Combining Eq.(4.20) with the definition of $a$ and $b$, we have:

$$
\begin{aligned}
a & \equiv R_{\text {res }} \\
& =R(\delta=0) \\
& =\frac{\left(r_{1}-r_{2}^{\prime}\right)^{2}}{\left(1-r_{1} r_{2}^{\prime}\right)^{2}} \\
b & \equiv R_{\text {non-res }} \\
& =R(\delta=\pi) \\
& =\frac{\left(r_{1}+r_{2}^{\prime}\right)^{2}}{\left(1+r_{1} r_{2}^{\prime}\right)^{2}}
\end{aligned}
$$

Their ratio $k$, in terms of $r_{1}$ and $r_{2}^{\prime}$ is:

$$
\begin{aligned}
k & \equiv \frac{a}{b} \\
& =\frac{\left(r_{1}-r_{2}^{\prime}\right)^{2}}{\left(r_{1}+r_{2}^{\prime}\right)^{2}} \frac{\left(1+r_{1} r_{2}^{\prime}\right)^{2}}{\left(1-r_{1} r_{2}^{\prime}\right)^{2}}
\end{aligned}
$$

where $r_{2}^{\prime}=r_{2} \sqrt{1-L}$ as defined earlier.

We want to solve for $L$ in terms of $r_{1}, r_{2}$ and $k$.

First solve for the quadratic equation of $r_{2}^{\prime}$ :

(i) when $r_{1}>r_{2}^{\prime}$,

$$
r_{2}^{\prime}=\frac{-(1+\sqrt{k})\left(1-r_{1}^{2}\right)}{2 r_{1}(1-\sqrt{k})}\left(1 \pm \sqrt{1+\frac{4 r_{1}^{2}(1-\sqrt{k})^{2}}{(1+\sqrt{k})^{2}\left(1-r_{1}^{2}\right)^{2}}}\right)
$$


(ii) when $r_{1}<r_{2}^{\prime}$,

$$
r_{2}^{\prime}=\frac{-(1-\sqrt{k})\left(1-r_{1}^{2}\right)}{2 r_{1}(1+\sqrt{k})}\left(1 \pm \sqrt{1+\frac{4 r_{1}^{2}(1+\sqrt{k})^{2}}{(1-\sqrt{k})^{2}\left(1-r_{1}^{2}\right)^{2}}}\right)
$$

To satisfy the physics requirement that $r_{2}^{\prime}$ should be positive, we only keep the positive solution:

(i) when $r_{1}>r_{2}^{\prime}$,

$$
r_{2}^{\prime}=\frac{-(1+\sqrt{k})\left(1-r_{1}^{2}\right)}{2 r_{1}(1-\sqrt{k})}\left(1-\sqrt{1+\frac{4 r_{1}^{2}(1-\sqrt{k})^{2}}{(1+\sqrt{k})^{2}\left(1-r_{1}^{2}\right)^{2}}}\right)
$$

(ii) when $r_{1}<r_{2}^{\prime}$,

$$
r_{2}^{\prime}=\frac{-(1-\sqrt{k})\left(1-r_{1}^{2}\right)}{2 r_{1}(1+\sqrt{k})}\left(1-\sqrt{1+\frac{4 r_{1}^{2}(1+\sqrt{k})^{2}}{(1-\sqrt{k})^{2}\left(1-r_{1}^{2}\right)^{2}}}\right)
$$

Now use Eq.(4.26) and 4.27 to solve for the intracavity loss $L$, and substitute $r_{1}$ and $r_{2}$ with $r_{1}=\sqrt{1-T_{1}}$ and $r_{2}=\sqrt{1-T_{2}}$ respectively:

(i) when $\sqrt{1-T_{1}}>r_{2} \sqrt{1-L}$ (i.e. $\left.L>\frac{T_{1}-T_{2}}{1-T_{2}}\right)$,

$$
L=1-\left[\frac{-T_{1}(1+\sqrt{k})}{2\left(1-T_{2}\right) \sqrt{1-T_{1}}(1-\sqrt{k})}\left(1-\sqrt{1+\frac{4\left(1-T_{1}\right)(1-\sqrt{k})^{2}}{T_{1}^{2}(1+\sqrt{k})^{2}}}\right)\right]^{2}
$$

(ii) when $\sqrt{1-T_{1}}<r_{2} \sqrt{1-L}$ (i.e. $L<\frac{T_{1}-T_{2}}{1-T_{2}}$ ),

$$
L=1-\left[\frac{-T_{1}(1-\sqrt{k})}{2\left(1-T_{2}\right) \sqrt{1-T_{1}}(1+\sqrt{k})}\left(1-\sqrt{1+\frac{4\left(1-T_{1}\right)(1+\sqrt{k})^{2}}{T_{1}^{2}(1-\sqrt{k})^{2}}}\right)\right]^{2}
$$


Eq.(4.28) and 4.29 are the intracavity loss calculation equations. Notice there are two cases for when $L$ is smaller or bigger than $\frac{T_{1}-T_{2}}{1-T_{2}}$. Plots of $L$ vs. $k$ can be obtained once the OPO is fixed and its two mirrors' $T_{1}, T_{2}$ are known. For the OPO of our experiment, we have $T_{1}=5 \%$ and $T_{2}=0$ (one-sided cavity), so plug in these two parameters we get the plot of $L$ vs. $k$ for our particular OPO shown in Figure 4.11:

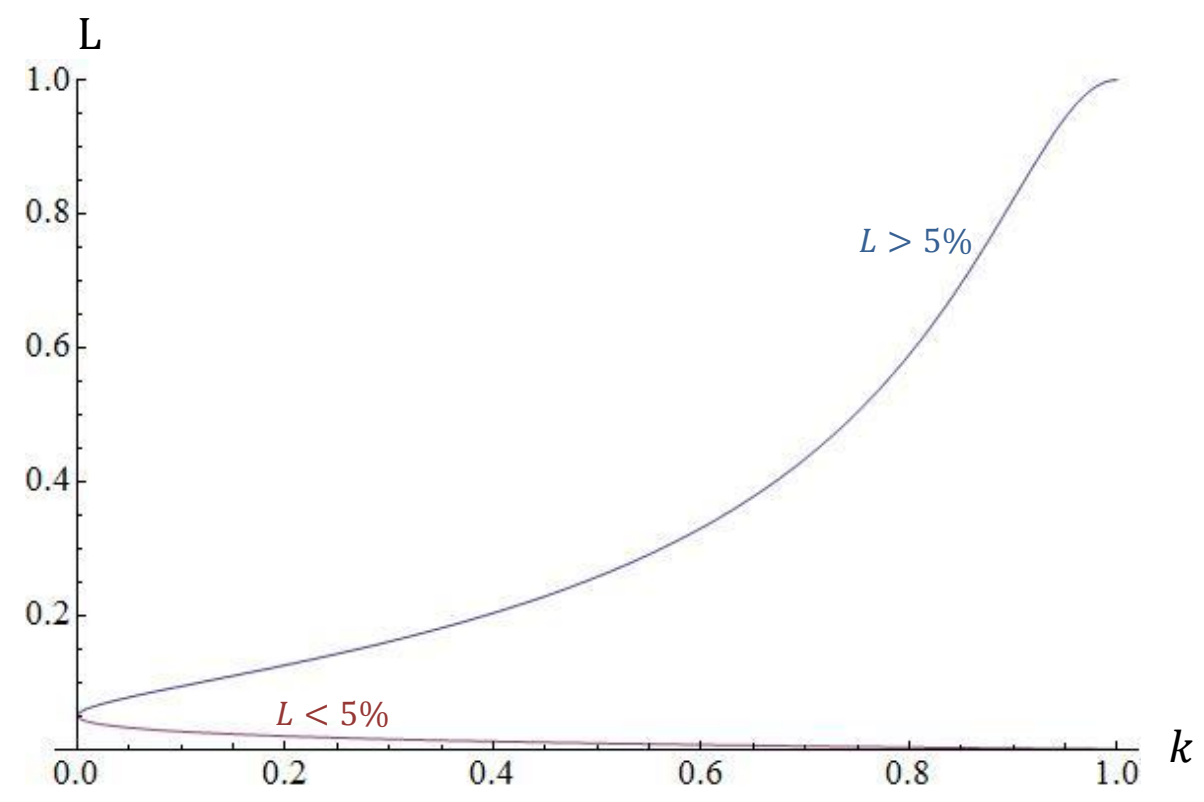

Figure 4.11: The intracavity loss $L$ versus $k$ for a one-sided OPO with output coupler $5 \%\left(T_{1}=5 \%, T_{2}=0\right.$ in Eq.(4.28) and 4.29). We can see that when $L$ is in the small region $\left(L<\frac{T_{1}-T_{2}}{1-T_{2}}=5 \%\right)$, the bigger the $k$ the smaller the intracavity loss; however, when $L$ is in the big region $\left(L>\frac{T_{1}-T_{2}}{1-T_{2}}=5 \%\right)$, the bigger the $k$ the smaller the intracavity loss.

For a given OPO, we just need to estimate which region the intracavity loss $L$ falls into according to the OPO cavity parameters $T_{1}$ and $T_{2}$, and then either use case (i) (Eq.(4.28)) or case (ii) (Eq.(4.29)) to calculate the intracavity loss based on the measured value of $k$. 


\subsection{Nonlinear optics}

As we mentioned earlier, there are two major constituents in an OPO: the optical cavity and the nonlinear medium. In our experiments, we use nonlinear crystal as the nonlinear medium. In this section, I will talk about the properties and behaviors of the nonlinear crystals. In the following text I will mainly use the book "Fundamentals of Photonics" by Saleh and Teich as a reference [57].

A nonlinear medium is characterized by a nonlinear relationship between the polarization density $\mathcal{P}$ and the electric field $\mathcal{E}$.

We can write the polarization density as a sum of linear and nonlinear $\left(\mathcal{P}_{N L}\right)$ parts:

$$
\begin{gathered}
\mathcal{P}=\epsilon_{0} \chi \mathcal{E}+\mathcal{P}_{N L}, \\
\mathcal{P}_{N L}=2 d \mathcal{E}^{2}+4 \chi^{(3)} \mathcal{E}^{3}+\ldots
\end{gathered}
$$

where $\epsilon_{0}$ is the permittivity of free space and $\chi$ is the electric susceptibility of the medium; $d$ and $\chi^{(3)}$ are coefficients describing the strength of the second- and third-order nonlinear effects respectively.

The wave equation derived from Maxwell's equations for an homogeneous, isotropic nonlinear dielectric medium can be written as:

$$
\nabla^{2} \mathcal{E}-\frac{1}{v^{2}} \frac{\partial^{2} \mathcal{E}}{\partial t^{2}}=\mu_{0} \frac{\partial^{2} \mathcal{P}_{N L}}{\partial t^{2}}
$$

where the speed of light in the medium $v=c_{0} / n=c_{0} / \sqrt{1+\chi}$ and the speed of 
light in vacuum $c_{0}=1 / \sqrt{\epsilon_{0} \mu_{0}}$.

The right side of equation 4.31 is regarded as the source that radiates inside the medium. We use the Born Approximation in the following calculations, where we use only the incident field $\mathcal{E}$ to calculate $\mathcal{P}_{N L}$ and neglect the $\mathcal{P}_{N L}$ produced by the new field. This approximation is adequate when the light intensity is sufficiently weak so that the nonlinearity is small.

\subsubsection{Second-order nonlinear optics}

For the second-order nonlinear optics, where the nonlinearities of order higher than the second are negligible, we have

$$
\mathcal{P}_{N L}=2 d \varepsilon^{2}
$$

And the electric field $\mathcal{E}$ needs to take the real part of an complex amplitude $\tilde{E}$,

$$
\begin{aligned}
\mathcal{E} & =\operatorname{Re}(\tilde{E})=\frac{1}{2}\left(\tilde{E}+\tilde{E}^{*}\right), \\
\mathcal{E}^{2} & =\frac{1}{4}\left(\tilde{E}^{2}+\tilde{E}^{*^{2}}+2 \tilde{E} \tilde{E}^{*}\right)
\end{aligned}
$$

For an electric field having two frequencies $\omega_{1}$ and $\omega_{2}$,

$$
\begin{aligned}
& \tilde{E}=E\left(\omega_{1}\right) e^{i \omega_{1} t}+E\left(\omega_{2}\right) e^{i \omega_{2} t}, \\
& \tilde{E}^{*}=E^{*}\left(\omega_{1}\right) e^{-i \omega_{1} t}+E^{*}\left(\omega_{2}\right) e^{-i \omega_{2} t}
\end{aligned}
$$


Thus,

$$
\begin{aligned}
\mathcal{E}^{2}= & \frac{1}{4}\left(\tilde{E}^{2}+\tilde{E}^{*^{2}}+2 \tilde{E} \tilde{E}^{*}\right) \\
= & \frac{1}{4}\left[E^{2}\left(\omega_{1}\right) e^{i 2 \omega_{1} t}+E^{2}\left(\omega_{2}\right) e^{i 2 \omega_{2} t}+2 E\left(\omega_{1}\right) E\left(\omega_{2}\right) e^{i\left(\omega_{1}+\omega_{2}\right) t}\right. \\
& +E^{* 2}\left(\omega_{1}\right) e^{-i 2 \omega_{1} t}+E^{* 2}\left(\omega_{2}\right) e^{-i 2 \omega_{2} t}+2 E^{*}\left(\omega_{1}\right) E^{*}\left(\omega_{2}\right) e^{-i\left(\omega_{1}+\omega_{2}\right) t} \\
& +2 E\left(\omega_{1}\right) E^{*}\left(\omega_{1}\right)+2 E\left(\omega_{2}\right) E^{*}\left(\omega_{2}\right)+ \\
& \left.2\left(E\left(\omega_{1}\right) E^{*}\left(\omega_{2}\right) e^{i\left(\omega_{1}-\omega_{2}\right) t}+E^{*}\left(\omega_{1}\right) E\left(\omega_{2}\right) e^{-i\left(\omega_{1}-\omega_{2}\right) t}\right)\right] \\
= & \frac{1}{4}\left\{2 \operatorname{Re}\left[E^{2}\left(\omega_{1}\right) e^{i 2 \omega_{1} t}\right]+2 \operatorname{Re}\left[E^{2}\left(\omega_{2}\right) e^{i 2 \omega_{2} t}\right]+4 \operatorname{Re}\left[2 E\left(\omega_{1}\right) E\left(\omega_{2}\right) e^{i\left(\omega_{1}+\omega_{2}\right) t}\right]\right. \\
& \left.+2\left[\left|E\left(\omega_{1}\right)\right|^{2}+\left|E\left(\omega_{2}\right)\right|^{2}\right]+4 \operatorname{Re}\left[E\left(\omega_{1}\right) E^{*}\left(\omega_{2}\right) e^{i\left(\omega_{1}-\omega_{2}\right) t}\right]\right\}
\end{aligned}
$$

Plug this into Eq.(4.32), we have

$$
\begin{aligned}
\mathcal{P}_{N L}= & 2 d \mathcal{E}^{2} \\
= & d\left\{\operatorname{Re}\left[E^{2}\left(\omega_{1}\right) e^{i 2 \omega_{1} t}\right]+\operatorname{Re}\left[E^{2}\left(\omega_{2}\right) e^{i 2 \omega_{2} t}\right]+2 \operatorname{Re}\left[2 E\left(\omega_{1}\right) E\left(\omega_{2}\right) e^{i\left(\omega_{1}+\omega_{2}\right) t}\right]\right. \\
& \left.+\left[\left|E\left(\omega_{1}\right)\right|^{2}+\left|E\left(\omega_{2}\right)\right|^{2}\right]+2 \operatorname{Re}\left[E\left(\omega_{1}\right) E^{*}\left(\omega_{2}\right) e^{i\left(\omega_{1}-\omega_{2}\right) t}\right]\right\} \\
= & \mathcal{P}_{N L}(0)+\operatorname{Re}\left[\mathcal{P}_{N L}\left(2 \omega_{1}\right) e^{i 2 \omega_{1} t}\right]+\operatorname{Re}\left[\mathcal{P}_{N L}\left(2 \omega_{2}\right) e^{i 2 \omega_{2} t}\right] \\
& +\operatorname{Re}\left[\mathcal{P}_{N L}\left(\omega_{+}\right) e^{i\left(\omega_{1}+\omega_{2}\right) t}\right]+\operatorname{Re}\left[\mathcal{P}_{N L}\left(\omega_{-}\right) e^{i\left(\omega_{1}-\omega_{2}\right) t}\right]
\end{aligned}
$$


with amplitudes

$$
\begin{aligned}
& \mathcal{P}_{N L}(0)=d\left[\left|E\left(\omega_{1}\right)\right|^{2}+\left|E\left(\omega_{2}\right)\right|^{2}\right] \\
& \mathcal{P}_{N L}\left(2 \omega_{1}\right)=d E^{2}\left(\omega_{1}\right) \\
& \mathcal{P}_{N L}\left(2 \omega_{2}\right)=d E^{2}\left(\omega_{2}\right) \\
& \mathcal{P}_{N L}\left(\omega_{+}\right)=2 d E\left(\omega_{1}\right) E\left(\omega_{2}\right) \\
& \mathcal{P}_{N L}\left(\omega_{-}\right)=2 d E\left(\omega_{1}\right) E^{*}\left(\omega_{2}\right)
\end{aligned}
$$

From Eq.(4.37) we can see that two optical waves of different frequencies can generate a third wave at the difference frequency or at the sum frequency through the second-order nonlinear medium.

If wave $\omega_{1}$ and $\omega_{2}$ are plane waves with wavevectors $\mathbf{k}_{\mathbf{1}}$ and $\mathbf{k}_{\mathbf{2}}$, so that $E\left(\omega_{1}\right)=$ $A_{1} e^{-i \mathbf{k}_{1} \cdot \mathbf{r}}$ and $E\left(\omega_{2}\right)=A_{2} e^{-i \mathbf{k}_{2} \cdot \mathbf{r}}$. According to Eq.(4.37), we have $\mathcal{P}_{N L}\left(\omega_{3}\right)=$ $\mathcal{P}_{N L}\left(\omega_{+}\right)=2 d E\left(\omega_{1}\right) E\left(\omega_{2}\right)=2 d A_{1} A_{2} e^{-i \mathbf{k}_{3} \cdot \mathbf{r}}$, where

$$
\omega_{1}+\omega_{1}=\omega_{3}
$$

and

$$
\mathrm{k}_{1}+\mathrm{k}_{2}=\mathrm{k}_{3}
$$

are known as Phase Matching Conditions. When they are satisfied, a third wave of a new frequency can be generated from the two incident waves. 
The amplitude of a wavevector is $k_{i}=n_{i} \omega_{i} / c_{0}$ where $n_{i}$ is the medium's index of refraction at frequency $\omega_{i}$ and $c_{0}$ is the speed of light in vacuum. Plug this relation in, and assume the collinear case (i.e. the three waves travel in the same direction), we obtain:

$$
\begin{aligned}
& \omega_{1}+\omega_{1}=\omega_{3}, \\
& \omega_{1} n_{1}+\omega_{2} n_{2}=\omega_{3} n_{3}
\end{aligned}
$$

\subsubsection{Quasi phase matching}

The phase matching conditions Eq.(4.40) are not necessarily satisfied for a dispersive medium where the indices of refraction at different frequencies are different $n_{1} \neq n_{2} \neq$ $n_{3}$

We could adjust the effective indices by tilting crystal axes (Critical phase matching)and/or find a special crystal and tune the crystal temperature (Non-critical phase matching) to satisfy the phase matching conditions. Here, I would like to talk about a more general and often-used method in our experiments for phase matching - Quasi phase matching.

According to Eq.(4.31), a new source $\mathcal{P}_{N L}\left(\omega_{3}\right)$ can generate a new wave of angular frequency $\omega_{3}$. It can be shown that for collinear (one-dimensional) case the intensity of the new wave:

$$
I_{3} \propto\left|d A_{1} A_{2} \int_{0}^{L} \exp (i \Delta k z) d z\right|^{2} \propto L^{2} \operatorname{sinc}^{2}(\Delta k L / 2)
$$


where $L$ is the nonlinear crystal's length during which the three waves interact in the $z$ direction (collinear direction) and $\Delta k=k_{3}-k_{1}-k_{2}$ is the phase mismatch. As the phase mismatch increases, the intensity of the new wave will decrease fast as the Sinc function has sharp slopes; Figure 4.12 shows the fast dropping tendency.

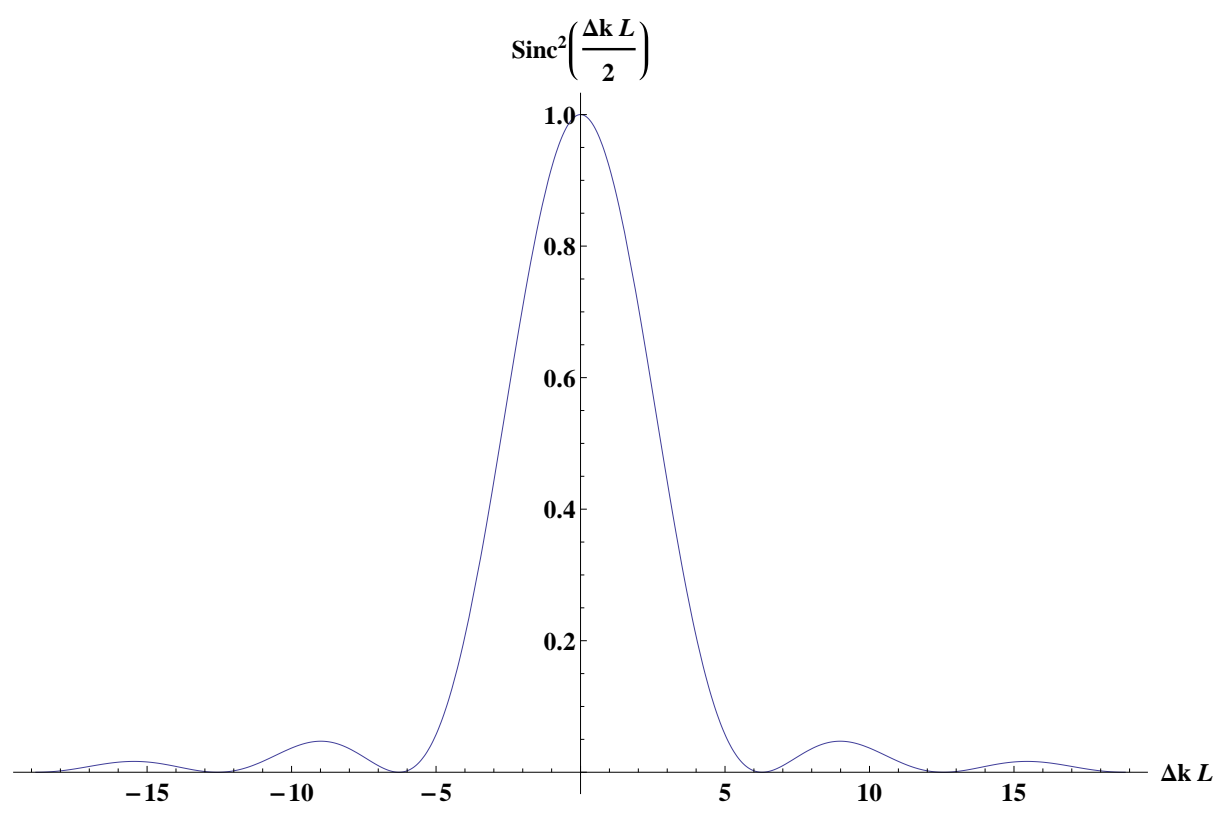

Figure 4.12: The three-wave mixing efficiency significantly reduces as the phase mismatch $\Delta k L$ increases.

Since the magnitude of the generated new wave is significantly reduced as the phase mismatch increases and sometimes phase match can be hard to achieve, we can use a medium with periodic nonlinearity to compensate the mismatch $\Delta k$.

Ideally the medium would have a position-dependent nonlinear coefficient $d(z)=$ $d_{0} e^{-i G z}$, with $G=\Delta k$. Plugging this back to Eq.(4.41) then the phase mismatch effect would be fully eliminated. However, such ideal grating is hard to achieve and in reality we can use a periodic nonlinear coefficient $d(z)$ described by the Fourier 
series for the quasi phase matching:

$$
d(z)=\sum_{m=-\infty}^{\infty} d_{m} \exp (-i 2 \pi m z / \Lambda)
$$

where $\Lambda$ is the period and $\left\{d_{m}\right\}$ is the Fourier coefficients.

Any of these Fourier components may be used for compensating the phase mismatch, just need to set $2 \pi m / \Lambda=G=\Delta k$ and solve for $\Lambda$ we get:

$$
\Lambda=m 2 \pi / \Delta k
$$

Usually the first order $m=1$ has the biggest Fourier coefficient $d_{1}$ so we often choose that and get $\Lambda=2 \pi / \Delta k$. For example, for a periodic pattern of the nonlinear coefficient $d(z)$ that alternates between two constants $+d_{0}$ and $-d_{0}$ at distances $\Lambda / 2$, it has the Fourier series: $d(z)=\sum_{m=o d d}\left[d_{0} \frac{2}{m \pi} \exp (-i m \Delta k z)\right]$. And the first order $m=1$ is used for compensating the phase mismatch, so the effective coefficient in this case is $d_{\mathrm{eff}}=d_{1}=\frac{2}{\pi} d_{0}$.

One way of fabricating the periodic nonlinear structure is called "poling". In our experiment we use a periodically-poled $\mathrm{KTiOPO}_{4}$ (KTP) crystal (PPKTP) as our nonlinear medium, as will see in Chapter 5. 


\section{Chapter 5}

\section{Experimental Entanglement Of 60 Modes In A Dual-rail Cluster State}

In this chapter I will describe the experimental generation of a 60-mode cluster state.

This is an experimental realization of multipartite entanglement in a single OPO. It is a significant result because the entanglement is a cluster state which has great importance for quantum computing. Reference [58] also has multipartite entanglement from a single OPO, but the resulting states are not cluster states which are necessary for one way quantum computing.

This carefully-engineered entangled state is the world record for the largest cluster state whose subsystems are all available simultaneously. There were larger entangled cluster state ( [37]) but the entanglement there is in the time domain (their modes are at different times while our modes are at different frequencies) and at an certain time only a small number of modes are accessible.

It is worth mentioning that the number of entangled modes 60 is limited only by the measuring parameters, rather than the generation techniques or entangled state 
itself. The total number of modes should actually be at least 6700 according to the nonlinear crystal phase matching bandwidth measurement [44]. 


\subsection{Previous proposals overview}

Before I get into the experimental details for the quantum wire generation, it is important to compare our experimental method with some of the old proposals $[31,32]$ and understand the compactness and beauty of the new design.

The main advantages of the current design are: no complicated pump spectrum needed, no multiple nonlinear interatcions required, and, no hard cutoff (termination) of the phase-matching curve needed. Those advantages helped simplify the experiments significantly.

The previously proposed method of generating a quantum wire cluster state $[31,32]$ involves a polarized pump spectrum containing 3 different frequencies with three $(z z z, z y y$ and $y z y$ with the first letter denotes the polarization of pump photon and the last two letters denote the polarizations of the created signal and idler photons) nonlinear interactions per frequency, or alternatively, pump spectrum containing 7 different frequencies with only one interaction per pump frequency. Both ways requires termination ${ }^{1}$.

Figure 5.1 shows the "crown" state proposed in references [31,32], notice when we measure $q$ for two nodes from the same macronode the crown can be cut open and becomes a dual-rail structured quantum wire instead.

In their proposals, to make such a crown state, one needs the adjacency matrix

\footnotetext{
${ }^{1}$ Later on an improvement was developed by our theory collaborator Nick Menicucci that only two pump frequencies (instead of three) are necessary and no termination was needed, however in that version the nonlinear crystal still requires three phase-matching conditions.
} 


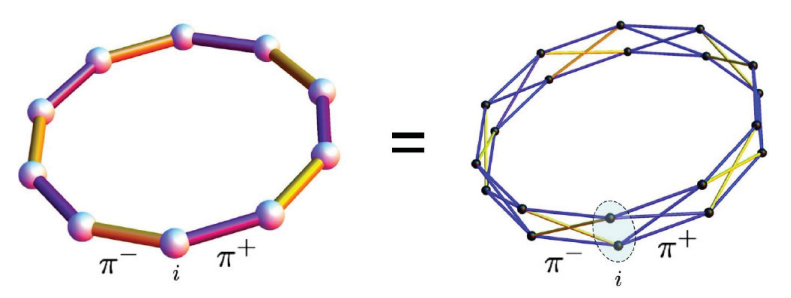

Figure 5.1: Crown quantum entangled state from [31,32]. Matrix-valued weights. Here a macronode (left) contains two nodes (right). Measuring $q$ for two nodes from the same macronode can cut the crown open into a dual-rail wire.

A of the form:

$$
\mathbf{A} \cong\left[\mathbf{0}, \ldots, \mathbf{0}, \pi^{+}, \mathbf{0} / \pi^{-} / \mathbf{0}, \ldots, \mathbf{0}, \pi^{+}, \mathbf{0}\right]
$$

The A matrix in Eq.(5.1) uses the block-Hankel form, and $\cong$ indicates equality up to renumbering of nodes, the zeros are $2 \times 2$ blocks of all zeros (the two zero groups $\mathbf{0}, \ldots, \mathbf{0}$ both have $N-3$ number of zeros, with $N$ being the total macronode number) and the $2 \times 2$ matrices $\pi^{+}, \pi^{-}$are defined below.

$$
\pi^{+}=\frac{1}{2}\left(\begin{array}{ll}
1 & 1 \\
1 & 1
\end{array}\right) \quad \pi^{-}=\frac{1}{2}\left(\begin{array}{cc}
1 & -1 \\
-1 & 1
\end{array}\right)
$$

The A matrix has three nonzero blocks indicating three pump frequencies [31,32], and each block $\left(\pi^{+}, \pi^{-}\right)$indicates there should be three equal-weight nonlinear interactions simultaneously given we adopt the pump polarization as the extra quantum number (other possibilities include wave vectors and transverse spatial modes which we don't use here). Specifically, if we use PPKTP as the nonlinear crystal in the OPO, we can use $z y y, y z y$ and $z z z$ interactions to achieve the $\pi^{+}, \pi^{-}$, with each pump frequency's polarization $\pm 45^{\circ}$ in the $\mathrm{YZ}$ plane respectively. This method requires a stringent selection on the participating modes of optical frequency comb, 
as any extra modes will yield additional, unwanted vertices and edges in the quantum graph we seek to create. This requires a hard cut off on the comb, also called termination ${ }^{1}$.

After a renumbering scheme, however, it can be converted into a fully Hankel form:

$$
\mathbf{A} \cong \frac{1}{2}\left[\mathbf{0}^{N-3}, 1,0,1, \mathbf{0}^{N-3}, 1,0 /-1 / \mathbf{0}^{N-3}, 1,0,1, \mathbf{0}^{N-3}, 1,0\right]
$$

where $\mathbf{0}^{n}$ represents a string of $n$ zeros.

From the fully Hankel form of adjacency matrix in Eq.(5.2) we can see that 7 pump frequencies are needed. After the renumbering scheme, the total number of pump frequency increases, but only one nonlinear interaction is needed as opposed to three interactions required for the 3-frequency pump in adjacency matrix 5.1.

As mentioned above, our new design doesn't require complicated pump spectrum (only two frequencies are needed), nor does it require complicated nonlinear interactions (only two $z z z$ phase-matched crystals are needed) or termination (the whole optical frequency comb is used). I will describe the new design and its experimental details and results in the following sections of this chapter. 


\section{$5.2 \quad$ Principles of experiments}

In this section I will explain the principles of the experimental generation of a dualrail quantum wire of 60 modes [34]. First I will introduce the qualitative concepts before giving the full derivation.

\subsubsection{Qualitative analysis}

The quantum wire generation starts with Einstein, Podolsky and Rosen (EPR) pairs [59]. Those EPR pairs are formed when a properly polarized pump is sent into an optical parametric oscillator (OPO). In particular, if we have one $z z z$ crystal in the OPO, as shown in Fig.5.2, when we send in a z-polarized pump, whose half frequency sits in the middle of two neighboring resonant modes, then many z-polarized EPR pairs will be generated (as shown in Figure 5.3).

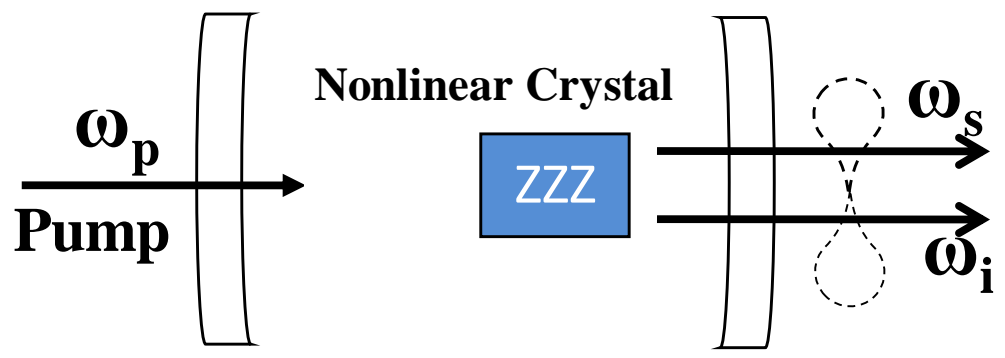

Figure 5.2: An OPO with a zzz nonlinear crystal. A z-polarized input pump can generate signal and idler pairs (EPR pairs) when the phase matching conditions are satisfied. The mirrors have high reflection at $1064 \mathrm{~nm}$ wavelength and high transmission at $532 \mathrm{~nm}$ wavelength.

If the frequencies for the pump, signal and idler are $\omega_{p}, \omega_{s}$ and $\omega_{i}$ respectively, 
the phase matching condition requires:

$$
\begin{aligned}
& \omega_{i}+\omega_{s}=\omega_{p} \\
& \mathbf{k}_{\mathbf{i}}+\mathbf{k}_{\mathbf{s}}=\mathbf{k}_{\mathbf{p}}
\end{aligned}
$$

where $\mathbf{k}$ is the wave vector whose direction is the signal propagating direction and amplitude $k \equiv \frac{\omega}{c}$

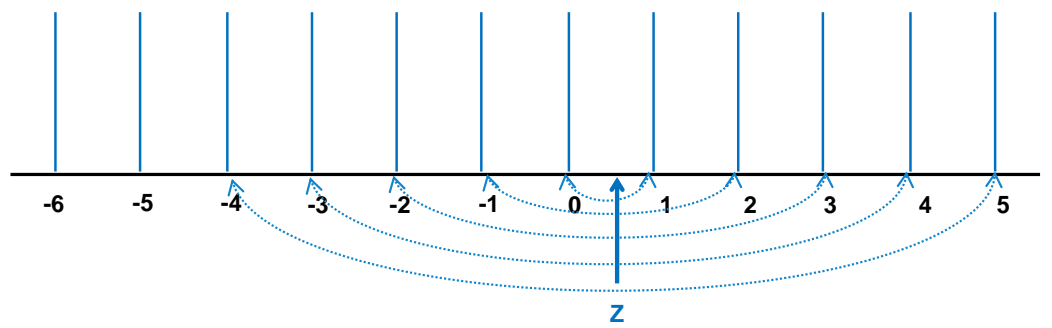

Figure 5.3: Optical Frequency Comb (OFC) of the OPO. The blue solid lines denote the $z$-polarized resonant modes; the vertical arrow marks the half-frequency of the pump; the curved arrows denote the $z z z$ EPR pairs.

Due to the phase matching requirements, all the EPR pairs are symmetric about the half-pump frequency $\frac{\omega_{0}+\omega_{1}}{2}$ (as shown in Figure 5.3 due to the phase matching requirements. If we reorganize the order of the resonant modes, as shown in Figure 5.4, all EPR pairs form a sequence. Notice that Figure 5.3 and Figure 5.4 only differ in the ordering of modes.

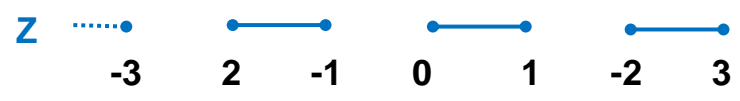

Figure 5.4: Reorganizing the $z z z$ EPR pairs to order them in a sequence.

So far what we have described is simple: one $z$-polarized optical pump generates 
many $z$-polarized EPR pairs through a $z z z$ phase matched nonlinear crystal inside an OPO. Now let us add another $z z z$ nonlinear crystal, except this time rotate it $90^{\circ}$ along the $x$-axis, so it becomes effectively yyy phase matched in the reference frame of the first crystal. The new OPO with the two orthogonal nonlinear crystals is shown in Figure 5.5.

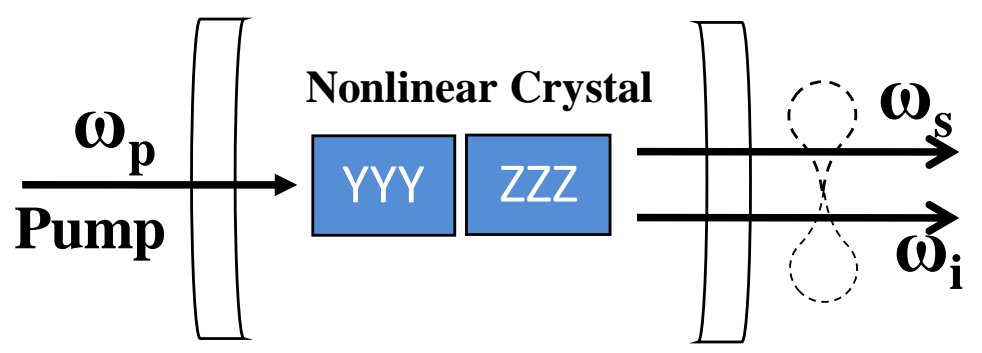

Figure 5.5: The OPO with two orthogonal nonlinear crystals so that they are yyy and $z z z$ phase matched respectively.

Now we have two crystals yyy and $z z z$ in the OPO, and we add in one more $y$-polarized pump as well. The $y$-polarized pump will only have nonlinear interaction with the yyy crystal, and the $z$-polarized pump will only have nonlinear interaction with the $z z z$ crystal, due to the orthogonality of the polarizations. Thus, the optical frequency comb looks like Figure 5.6, where the red color denotes the $y$-polarization and blue color denotes the $z$-polarization.

Again, if we reorganize the frequencies in the same order as that of Figure 5.4, then we have two sets of EPR pairs in two sequences, one for each polarization, as shown in Figure 5.7. The way to create a quantum wire is, intuitively, to connect both sequences. Our way to connect them, simply put, is to make the use of the beam splitter. 


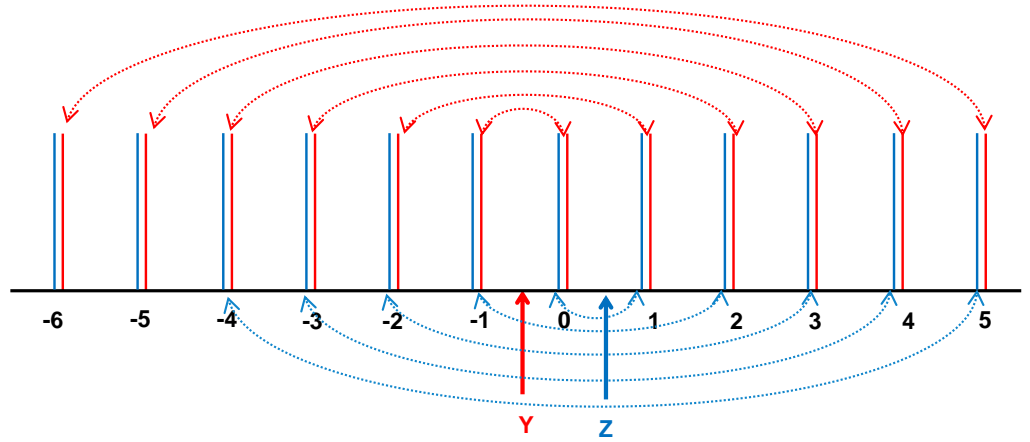

Figure 5.6: The OFC for two polarizations. The blue (red) color denotes the $z(y$-)polarized resonant modes and half frequency of the pumps.

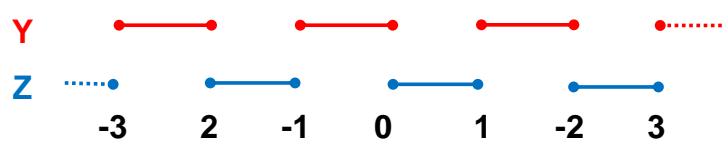

Figure 5.7: Reorganize the yyy EPR pairs to align them in an array as well. Now both polarizations' EPR pairs form an array.

As shown in Figure 5.7, at one frequency there are two modes - $y$ - and $z$-polarized modes, and when we pass them in a beam splitter (experimentally we use a half-wave plate (HWP) and a polarizing beam splitter to achieve that), the two modes with the same frequency will interact at the outputs. As all mode pairs with the same frequency will do the same, the separate EPR pairs get entangled into a dual-rail wire. This is shown in Figure 5.8. The derivations will be shown next. 

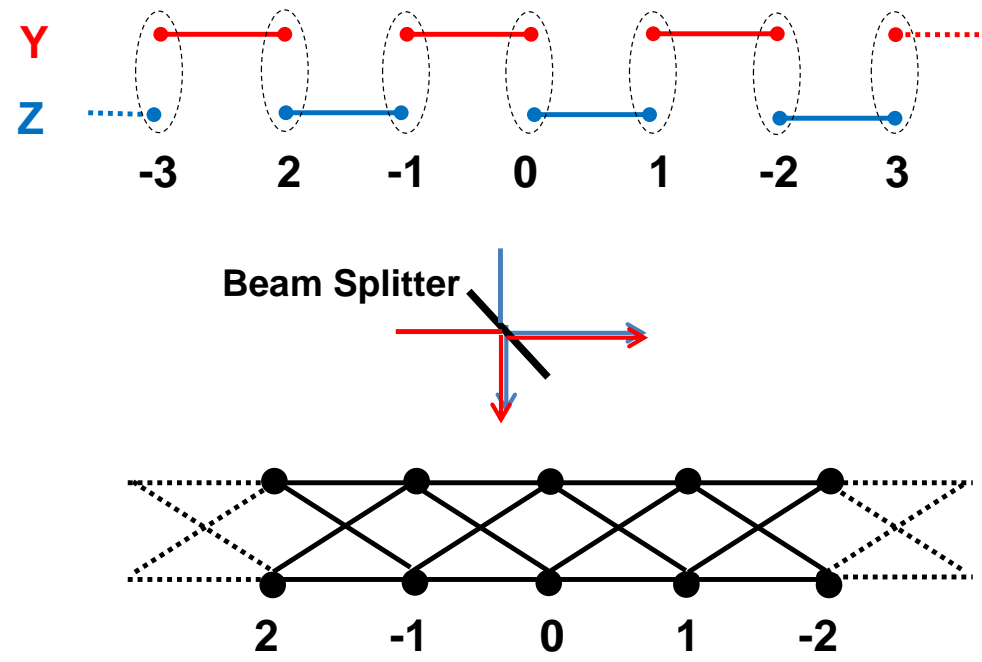

Figure 5.8: The separate $z z z$ and yyy EPR pairs connect into a dual-rail structured wire after the beam splitter. 


\subsubsection{Theoretical analysis}

To show how this works, we label the modes in the QOFC by integer $n$ such that $\omega_{n}=\omega_{0}+n \Delta \omega$, with $\omega_{0}$ an arbitrary origin and $\Delta \omega$ the OPO free spectral range (FSR). As shown in Figure5.9(a).
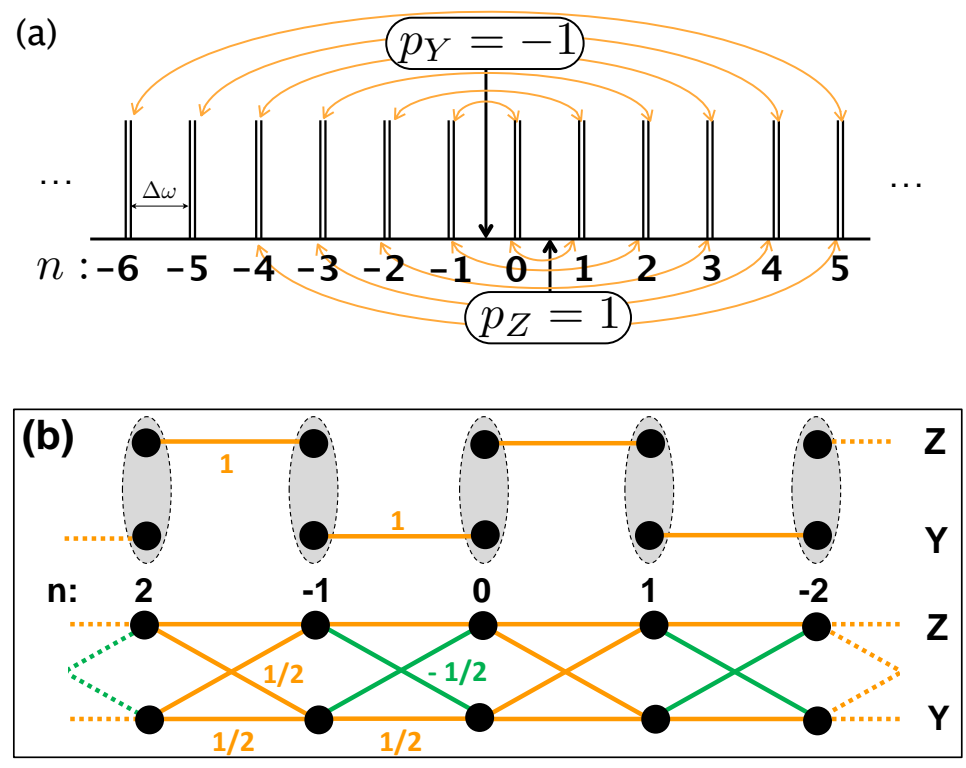

Figure 5.9: Generation of a dual-rail quantum wire in the QOFC (with pump index and weights labeled). (a) EPR pairs created by $z z z$ and yyy interactions in the QOFC of a polarization-degenerate OPO (here for color simplicity at each frequency $n$ the $z$ and $y$ modes are denoted by the double black lines). The vertical arrows mark the half-frequencies of the pumps; the curved arrows denote the $z z z$ (bottom) and yyy (top) EPR pairs. (b) Quantum graph states [60]: The initial EPR pairs from the OPO (top) turn, after a single beam splitter (grey ellipses), into a dual-rail CV cluster state (bottom), whose $\pm 1 / 2$-weight edges are color-coded.

The parametric down conversion (PDC) phasematching condition for EPR pair $\left(n_{1}, n_{2}\right)$ gives $\omega_{p}=\omega_{n_{1}}+\omega_{n_{2}}=2 \omega_{0}+p \Delta \omega$, where $p=n_{1}+n_{2}$ is the pump index. For $\left|p_{y}-p_{z}\right|=2$, i.e., pump frequencies differing by exactly twice the OPO FSR, all EPR pairs concatenate into the frequency sequence $(\ldots,-2,1,0,-1,2,-3, \ldots)$ that 
extends to the whole phasematching bandwidth in the QOFC.

To verify entanglement, we measured the joint squeezed operators called variancebased entanglement witnesses [61] or nullifiers [53], which are the solutions of our OPO's Heisenberg equations. To better understand the derivation of the nullifiers, we redraw the OFC with the pump index $p_{y}$ and $p_{z}$ labeled (Fig. 5.9(a)) and its corresponding quantum wire with weights (Fig. 5.9(b)).

Nullifiers are directly related to the stabilizers of the generated cluster state [62] and are also used in a more general entanglement check by the van Loock-Furusawa criterion [63]. Their derivation in the Heisenberg picture (see also Refs. [37, 46, 64]) uses the OPO's interaction-picture Hamiltonian,

$$
H=i \hbar\left[\kappa_{z} \sum_{k=n_{z}}^{\frac{N}{2}} a_{k}^{(z) \dagger} a_{p_{z}-k}^{(z) \dagger}+\kappa_{y} \sum_{l=n_{y}}^{\frac{N}{2}} a_{l}^{(y) \dagger} a_{p_{y}-l}^{(y) \dagger}\right]+\text { H.c. },
$$

where $n_{z, y}=\left\lceil\frac{p_{z, y}}{2}\right\rceil$.

and the Heisenberg equation,

$$
\frac{d a(t)}{d t}=\frac{1}{i \hbar}[a(t), H]
$$

The solutions are the EPR nullifiers

$$
\begin{aligned}
& {\left[Q_{n}^{(j)}-Q_{p_{j}-n}^{(j)}\right] e^{-r_{j}}} \\
& {\left[P_{n}^{(j)}+P_{p_{j}-n}^{(j)}\right] e^{-r_{j}}}
\end{aligned}
$$

where $j=y, z$, and $r_{j}=\kappa_{j} t$ are the squeezing parameters. 
They are called nullifiers because as the time $t$ goes to infinite, their values will vanish as we can see in Eq. (5.7).

A beam splitter (achieved by a HWP at $22.5^{\circ}$ from the crystal's axes followed by a polarizing beam splitter aligned with the crystal's axes) generates a $45^{\circ}$ polarization rotation on the annihilation operators $\left(a_{n}^{(z)}, a_{n}^{(y)}\right)^{T}$, in the matrix form

$$
\frac{1}{\sqrt{2}}\left(\begin{array}{cc}
1 & 1 \\
1 & -1
\end{array}\right)
$$

Thus the new operators become (the factor $\frac{1}{\sqrt{2}}$ is trivial here, so we will ignore it):

$$
\begin{aligned}
& a_{n}^{(z)} \rightarrow a_{n}^{(z)}+a_{n}^{(y)} \\
& a_{n}^{(y)} \rightarrow a_{n}^{(z)}-a_{n}^{(y)}
\end{aligned}
$$

So we have,

$$
\begin{aligned}
& Q_{n}^{(z)} \rightarrow Q_{n}^{(z)}+Q_{n}^{(y)} \\
& P_{n}^{(z)} \rightarrow P_{n}^{(z)}+P_{n}^{(y)} \\
& Q_{n}^{(y)} \rightarrow Q_{n}^{(z)}-Q_{n}^{(y)} \\
& P_{n}^{(y)} \rightarrow P_{n}^{(z)}-P_{n}^{(y)}
\end{aligned}
$$


Plugging these in Eq.(5.7), it then transforms the EPR nullifiers into

$$
\begin{aligned}
& Q_{p_{z}-n, n}\left(r_{z}\right)=\left\{\left[Q_{n}^{(z)}+Q_{n}^{(y)}\right]-\left[Q_{p_{z}-n}^{(z)}+Q_{p_{z}-n}^{(y)}\right]\right\} e^{-r_{z}} \\
& P_{p_{z}-n, n}\left(r_{z}\right)=\left\{\left[P_{n}^{(z)}+P_{n}^{(y)}\right]+\left[P_{p_{z}-n}^{(z)}+P_{p_{z}-n}^{(y)}\right]\right\} e^{-r_{z}} \\
& Q_{p_{y}-n, n}\left(r_{y}\right)=\left\{\left[Q_{p_{y}-n}^{(z)}-Q_{p_{y}-n}^{(y)}\right]-\left[Q_{n}^{(z)}-Q_{n}^{(y)}\right]\right\} e^{-r_{y}} \\
& P_{p_{y}-n, n}\left(r_{y}\right)=\left\{\left[P_{p_{y}-n}^{(z)}-P_{p_{y}-n}^{(y)}\right]+\left[P_{n}^{(z)}-P_{n}^{(y)}\right]\right\} e^{-r_{y}} .
\end{aligned}
$$

Assuming (a deviation from this case will be analyzed at the end of this section) $r_{z}=r_{y}=r$

When $n$ is even, taking the sum and difference of Eq.(5.15) and (5.17), and applying a Fourier transform - a.k.a. a local $\frac{\pi}{2}$ optical phase shift (as described in Chapter3) - to mode $n$ and all the even modes (here $n \pm 2$ ) yields the standard CV graph nullifiers (refer to Section 3.4.3). (in our case $p_{y}$ and $p_{z}$ are both odd, so the modes $p_{y}-n$ and $p_{z}-n$ are odd when $n$ is even thus no Fourier transform should apply on those modes):

$$
\begin{aligned}
& {\left[P_{n}^{(z)}-\frac{1}{2}\left(Q_{p_{z}-n}^{(y)}+Q_{p_{z}-n}^{(z)}+Q_{p_{y}-n}^{(z)}-Q_{p_{y}-n}^{(y)}\right)\right] e^{-r}} \\
& {\left[P_{n}^{(y)}-\frac{1}{2}\left(Q_{p_{z}-n}^{(y)}+Q_{p_{z}-n}^{(z)}-Q_{p_{y}-n}^{(z)}+Q_{p_{y}-n}^{(y)}\right)\right] e^{-r}}
\end{aligned}
$$

When $n$ is odd, taking the sum and difference of Eq.(5.16) and (5.18) and again applying the corresponding Fourier transform to the even nodes (here $p_{y}-n$ and 
$p_{z}-n$ are even) yields the nullifiers for graph nodes of odd parity:

$$
\begin{aligned}
& {\left[P_{n}^{(z)}-\frac{1}{2}\left(Q_{p_{z}-n}^{(y)}+Q_{p_{z}-n}^{(z)}+Q_{p_{y}-n}^{(z)}-Q_{p_{y}-n}^{(y)}\right)\right] e^{-r}} \\
& {\left[P_{n}^{(y)}-\frac{1}{2}\left(Q_{p_{z}-n}^{(y)}+Q_{p_{z}-n}^{(z)}-Q_{p_{y}-n}^{(z)}+Q_{p_{y}-n}^{(y)}\right)\right] e^{-r}}
\end{aligned}
$$

According to the cluster state definition $\mathbf{P}-\mathbf{A Q}$ (with matrix $\mathbf{A}$ indicating the graph as defined in Eq.(3.42)), the nullifiers 5.19 to 5.22 corresponds to the dual-rail quantum wire cluster state shown in Figure 5.9(b). Thus we have shown mathematically the generation of dual-rail cluster state from a QOFC.

\subsubsection{Analysis of unequal squeezing parameters}

In this section we investigate the consequences of $r_{z} \neq r_{y}$ to first order. We have the initial nullifiers, in case $n=0, p_{z}=1, p_{y}=-1$,

$$
\begin{gathered}
{\left[\left(Q_{0}^{z}+Q_{0}^{y}\right)-\left(Q_{1}^{z}+Q_{1}^{y}\right)\right] e^{-r_{z}}} \\
{\left[\left(P_{0}^{z}+P_{0}^{y}\right)+\left(P_{1}^{z}+P_{1}^{y}\right)\right] e^{-r_{z}}} \\
{\left[\left(Q_{-1}^{z}-Q_{-1}^{y}\right)-\left(Q_{0}^{z}-Q_{0}^{y}\right)\right] e^{-r_{y}}} \\
{\left[\left(P_{-1}^{z}-P_{-1}^{y}\right)+\left(P_{0}^{z}-P_{0}^{y}\right)\right] e^{-r_{y}} .}
\end{gathered}
$$

Assuming $r_{z, y}=r \pm \varepsilon, \varepsilon \ll r$, taking the sum and difference of Eq 5.23 and 5.25, and applying a Fourier transform, a.k.a. a local $\frac{\pi}{2}$ optical phase shift, to mode 0 yields, 
to first order in $\varepsilon$

$$
\begin{aligned}
& \left\{P_{0}^{z}-\varepsilon P_{0}^{y}-\frac{1}{2}\left[(1-\varepsilon)\left(Q_{1}^{y}+Q_{1}^{z}\right)+(1+\varepsilon)\left(Q_{-1}^{z}-Q_{-1}^{y}\right)\right]\right\} e^{-r} \\
& \left\{P_{0}^{y}-\varepsilon P_{0}^{z}-\frac{1}{2}\left[(1-\varepsilon)\left(Q_{1}^{y}+Q_{1}^{z}\right)-(1+\varepsilon)\left(Q_{-1}^{z}+Q_{-1}^{y}\right)\right]\right\} e^{-r}
\end{aligned}
$$

So the effect of unbalanced squeezing is a spurious correlation between the $0 z$ and $0 y$ modes, as well as edge weights of unequal magnitude in the rest of the graph. While these effects can be made arbitrarily small in our experiment by tuning the relative pump intensities, they ought to be kept in mind when evaluating the performance of future quantum processing applications.

\subsubsection{Generation to multiple wires}

I have derived the generation of one dual-rail quantum wire. A remarkable feauture of our frequency-domain implementaion is that merely tuning the pump spacing $\mid p_{y}-$ $p_{z} \mid=2 m$, yields $m$ disjoint frequency sequences and hence $m$ independent dual-rail cluster states. See Figure 5.10 for $m=2$.

Similarly, if we tune the pump spacing further more wires can be generated with the otherwise same setup. 


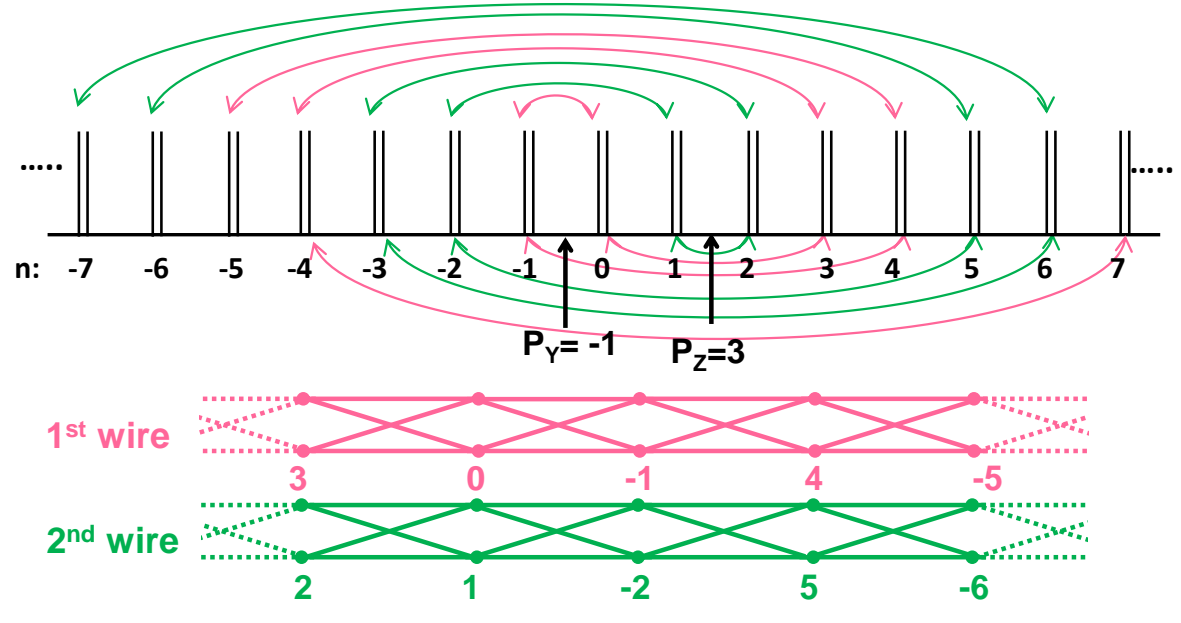

Figure 5.10: Generation of two dual-rail quantum wires. The only difference with the one wire case is that the pump frequency difference is $4 \Delta \omega$ instead of $2 \Delta \omega$. The frequency sequences of the wires are totally distinct: $(\ldots,-8,7,-4,3,0,-1,4,-5,8, \ldots)$ for the pink wire and $(\ldots,-7,6,-3,2,1,-2,5,-6,9, \ldots)$ for the green wire. 


\subsection{Experimental setup and equipment}

\subsubsection{Experimental setup}

Our polarization-degenerate OPO had a bowtie cavity (Figure 5.11) of FSR $\Delta \omega=$ 945.66 MHz. The OPO cavity length was actively stabilized by locking to a weak counterpropagating beam via a Pound-Drever-Hall (PDH) servo loop. The cavity eigenmode had two waists, where we placed the two PPKTP crystals, one $(31 \mu \mathrm{m})$ between the curved mirrors and one $(131 \mu \mathrm{m})$ between the flat mirrors. Great care was taken to suppress polarization crosstalk between the crystals as well as resonant retroreflection from the OPO cavity.

Two frequency-doubled, ultrastable continuous-wave (cw) Nd:YAG lasers, of frequency linewidth $1 \mathrm{kHz}$ at $532 \mathrm{~nm}$, were used for the pump fields. The lasers were phaselocked together at a frequency difference $2 m \Delta \omega$, with $m=1$ or $m=2$. The two pump beams entered the OPO through different paths to make a single pass through the yyy and zzz PPKTP crystals separately. To realize $r_{y}=r_{z}$, the pump powers were independently adjusted to compensate for the different waists at each crystal.

To test the dual-rail wire structure, the 4-mode nullifiers were measured, at all frequencies, by a two-tone balanced homodyne detection (BHD) system whose local oscillator (LO) was provided by another Nd:YAG cw laser, phaselocked at (and sometimes offset from) the half frequency of one of the pumps. The two LO tones were then generated by an electro-optic phase modulator (EOM) at a frequency $\Omega=\left(n+\frac{1}{2}\right) \Delta \omega$, such that $\omega_{L O}+\Omega=\omega_{n}$ and $\omega_{L O}-\Omega=\omega_{p_{y}-n}$, for example. The EOM's $\Omega_{\max }=14$ 


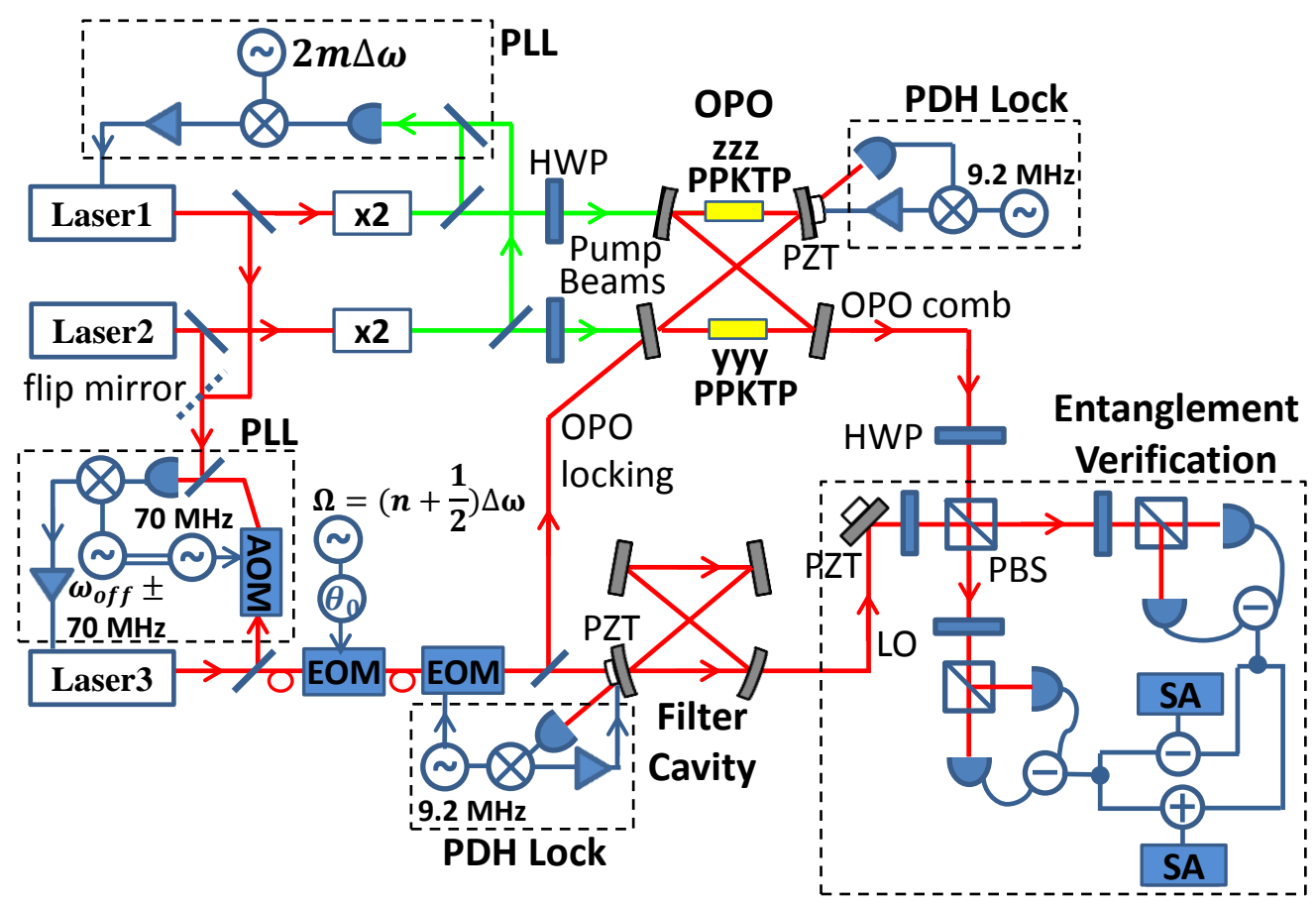

Figure 5.11: Experimental setup. PLL: phase-lock loop; HWP: half wave plate; PZT: piezoelectric transducer; PBS: polarizing beam splitter; SA: spectrum analyzer; AOM: acousto-optic modulator; EOM: electro-optic modulator; PDH: Pound-DreverHall lock loop.

GHz bandwidth yielded $n_{\max }=14$, i.e., $2 \times 15$ measurable modes (starting from $n=0$ ) for each polarization. (Replacing this EOM system with two phaselocked, widely tunable $1064 \mathrm{~nm}$ diode lasers will give us access to the aforementioned 6,700 modes instead of the current 60.) The first-order EOM sidebands were subsequently bandpass-filtered by a cavity of FSR $\Delta \omega$, PDH-locked on the LO laser. The LO phase was adjusted by a piezoelectric transducer mirror and an electronic splitter/combiner network was used to form the nullifier signals. 


\subsubsection{Experimental equipment}

The PPKTP crystals were provided by Raicol, Inc., and were $10 \mathrm{~mm}$-long, $x$-cut, periodically poled at $9 \mu \mathrm{m}$ so as to quasiphasematch [65-67] zzz PDC. They were antireflection coated by Advanced Thin Films at $1064 \mathrm{~nm}$ (for both polarizations) and $532 \mathrm{~nm}$, and mounted oriented at $90^{\circ}$ from each other in the $(y z)$ plane. Each crystal was temperature-controlled to a few tenths of a millidegree by using Wavelength Electronics servo loop chips, and the temperature was tuned within the phase matching bandwidth so as to equate the optical paths at each polarization.

The OPO mirrors were fabricated by Advanced Thin Films. The cavity was formed by two concave mirrors (50 mm radius) and two flat mirrors, one of which the output coupler of transmissivities of $5 \%$ at $1064 \mathrm{~nm}$ and $0.05 \%$ at $532 \mathrm{~nm}$. All other mirrors have transmissivities of near-zero at $1064 \mathrm{~nm}$ and near-unity at $532 \mathrm{~nm}$. The OPO cavity length was actively stabilized by locking it to a weak counterpropagating LO beam via a Pound-Drever-Hall servo loop. Our servo loops were all built in house, except for one Vescent D2-125 module that was occasionally used. The bowtie resonator had two beam waists, of $31 \mu \mathrm{m}$ (between the curved mirrors) and $131 \mu \mathrm{m}$ (between the flat mirrors), where the two PPKTP nonlinear crystals were placed.

Great care was taken to verify that there is no polarization crosstalk between the two crystals by generating the second harmonic of a $1064 \mathrm{~nm}$ seed laser beam modematched to the OPO cavity, and by checking the absence of $y(z)$-polarized radiation at $532 \mathrm{~nm}$ in the presence $z(y)$-polarized seed at $1064 \mathrm{~nm}$. 
We also noticed that the ring OPO, when seeded by a laser beam, exhibited a retroreflected beam from a cavity mode counterpropagating to the seed mode, and whose power can reach the order of $10 \%$ of the incident seed's power. We found that this counterpropagating mode stemmed from residual reflections on the crystal faces, which created an intra-OPO system of coupled cavities. We managed to minimize this resonant retroreflection from the OPO by slightly angling the crystals in the OPO cavity.

Two frequency-doubled, ultrastable continuous-wave Nd:YAG lasers (Innolight "Diabolo" 1W), of frequency linewidth $1 \mathrm{kHz}$ at $532 \mathrm{~nm}$, were used for the pump fields. The lasers were phaselocked together at a frequency difference $2 m \Delta \omega$, with $m=1,2$. This was achieved by a standard superheterodyne setup: one of the lasers was controlled via its laser crystal piezotransducer so as to phaselock the lasers' beat note to the stable radiofrequency delivered by an Agilent E8247C CW signal generator. The two pump beams then entered the OPO through different paths to access the yyy and $z z z$ PPKTP crystals separately.

The two-tone balanced homodyne detection system used 95\%-efficient JDSU ETX500T InGaAs photodiodes. Another Nd:YAG continuous-wave laser (JDSU Lightwave Electronics Model 126) provided the LO and the OPO locking beam. The LO frequencies were generated by phase EOM sidebands from a Photline NIR-MPX modulator, driven by a Hittite HMC-T2100 generator. The filter cavity was locked such that the first order harmonics of the LO sideband will transmit to beat with the particular 
frequencies we intend to measure, and the zero and second order will reflect.

The LO laser was phaselocked to one of the fundamental pump lasers by shifting the LO frequency by $70 \mathrm{MHz}$ with an IntraAction ATM-801A2 acousto-optic modulator and locking the resulting beat note to a Hewlett Packard 8648A signal generator, phaselocked to another, identical, signal generator which was the $70 \mathrm{MHz}$ source. That way, having both generators at $70 \mathrm{MHz}$ ensured both lasers could be locked at the same frequency. When we needed a frequency offset to check the graph, we simply shifted the frequency of the first generator.

The squeezing measurements were performed on an Agilent E4402B spectrum analyzer, the detection network being made of Mini-Circuits components.

\subsubsection{Basic experimental methods Pound-Drever-Hall (PDH) locking}

Pound-Drever-Hall locking is a powerful technique to stabilize the laser frequency [68]. It can be used to lock the laser's frequency to a certain mode of an optical cavity, or the other way, to lock an optical cavity so that one of its resonant modes matches with a certain laser frequency. In our experiment we used two PDH lockings to lock the filter cavity and OPO to the same laser frequency so that their combs overlap.

The conceptual foundations of frequency modulation (FM) spectroscopy and PDH laser locking are quite similar. PDH employs FM spectroscopy to derive its error signal; hence, FM spectroscopy is part of PDH locking. I will introduce the FM spectroscopy first. [69]. 
Bjorklund introduced the FM spectroscopy in optics [69], and I will summarize his paper here.

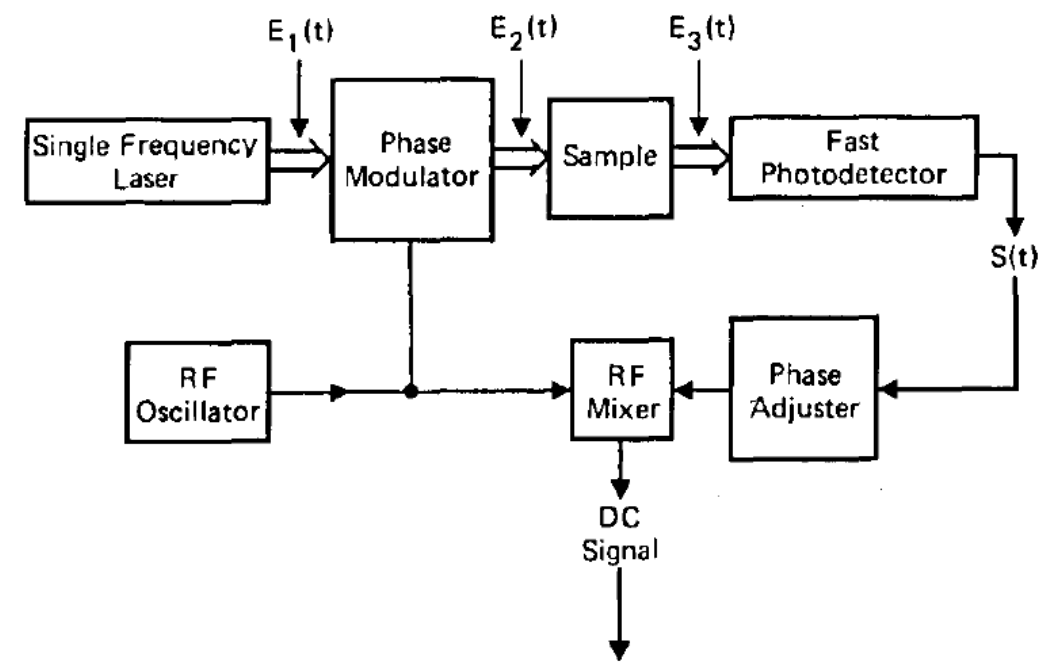

Figure 5.12: Typical experimental arrangement for FM spectroscopy (from [69]).

Figure 5.12 is a typical experimental arrangement for FM spectroscopy. A singlefrequency laser has frequency $\omega_{c}$ and its electric field is given by $E_{1}(t)=\frac{1}{2} \tilde{E}_{1}(t)+$ c.c., where $\tilde{E}_{1}(t)=E_{0} \exp i \omega_{c} t$, and $\omega_{c}$ denotes the carrier frequency. After passing the frequency modulator (phase modulator) driven by a rf signal at frequency $\omega_{m}$, the beam becomes:

$$
E_{2}(t)=\frac{E_{0}}{2} \sum_{n=-\infty}^{\infty} J_{n}(M) e^{i\left(\omega_{c}+n \omega_{m}\right) t}+c . c .
$$

where $M$ is the modulation index in radians and the $J_{n}$ are Bessel functions of order $n$. Assume $M \ll 1$, then $J_{0} \cong 1, J_{ \pm 1}(M) \cong \pm M / 2$, and all other terms vanish. Thus, $E_{2}(t)$ becomes:

$$
E_{2}(t) \cong \frac{E_{0}}{2}\left[e^{i \omega_{c} t}+\frac{M}{2} e^{i\left(\omega_{c}+\omega_{m}\right) t}-\frac{M}{2} e^{i\left(\omega_{c}-\omega_{m}\right) t}\right]+c . c .
$$


Define the sample's transmission function

$$
T_{n}=e^{-\delta_{n}-i \phi_{n}}
$$

where $n=-1,0,1$ denotes the values at $\omega_{c}-\omega_{m}, \omega_{m}$ and $\omega_{c}+\omega_{m}$ respectively, and $\delta_{n}$ and $\phi_{n}$ describes the attenuation (or gain) and dispersion experienced by each frequency component respectively. The transmitted field has:

$$
\tilde{E}_{3}(t)=E_{0}\left[T_{0} e^{i \omega_{c} t}+T_{1} \frac{M}{2} e^{i\left(\omega_{c}+\omega_{m}\right) t}-T_{-1} \frac{M}{2} e^{i\left(\omega_{c}-\omega_{m}\right) t}\right]
$$

The intensity on the photodetector is given by $I_{3}(t)=c \epsilon_{0} n\left|\tilde{E}_{3}(t)\right|^{2} / 2$. Dropping the second order terms in $M$, the result is:

$$
\begin{aligned}
I_{3}(t) & \propto E_{0}{ }^{2} e^{-2 \delta_{0}}\left\{1+\left[e^{\delta_{0}-\delta_{1}} \cos \left(\phi_{1}-\phi_{0}\right)-e^{\delta_{0}-\delta_{-1}} \cos \left(\phi_{0}-\phi_{-1}\right)\right] M \cos \omega_{m} t\right. \\
& \left.+\left[e^{\delta_{0}-\delta_{1}} \sin \left(\phi_{1}-\phi_{0}\right)-e^{\delta_{0}-\delta_{-1}} \sin \left(\phi_{0}-\phi_{-1}\right)\right] M \sin \omega_{m} t\right\} .
\end{aligned}
$$

If the arguments of exponential terms and cos, sin terms in Eq. (5.33) are all small compared with 1, then Eq. (5.33) simplifies to:

$$
I_{3}(t) \propto E_{0}^{2} e^{-2 \delta_{0}}\left[1+\left(\delta_{-1}-\delta_{1}\right) M \cos \omega_{m} t+\left(\phi_{1}+\phi_{-1}-2 \phi_{0}\right) M \sin \omega_{m} t\right] .
$$

The $\cos \omega_{m} t$ component is proportional to the difference in loss experienced by the upper and lower sidebands, whereas the $\sin \omega_{m} t$ component is proportional to the difference between the phase shift experienced by the carrier and the average of the phase shifts experienced by the sidebands. Note that in order to get Eq. (5.34) from Eq. (5.33), the assumptions for the smaller than 1 attenuation and phase shift 
differences were made. But without these assumptions (i.e. using Eq. (5.33) directly), the qualitative characteristics of the results still holds; it just cannot be seen as easily.

When the modulation frequency changes relative to the width of the spectral feature of interest, the $\cos \omega_{m} t$ and $\sin \omega_{m} t$ components' shapes change accordingly. To better illustrate this, we look at the example of when the spectral feature of interest, i.e. the "sample", is an optical cavity whose linewidth is $\Gamma$ (and the modulation frequency is $\left.\omega_{m}\right)$. Thus, the quantities $T_{n}$ in Eq.(5.32) is the optical cavity's reflectivity $R$. All the figures 5.13 were drawn based on Eq.(5.32).

From the simulation figures 5.13, it is clear that as the relative size of the modulation frequency and the linewidth of the system changes, the shapes and properties of the final signals change as well.

PDH locking (see Figure 5.14 for a typical layout of PDH locking loop) derives its error signal from FM spectroscopy and, in particular, the "sample" in the PDH locking system is an optical cavity, and the optical cavity's reflectivity or transmission signal, which is frequency dependent, serves as the spectral feature of the "sample". The DC signal after demodulation (which is a process where the signal obtained after the "sample" mixes with the signal used to drive the modulator), is the "error signal" to feedback either to the single frequency laser for locking the laser frequency to one of the resonant modes of the optical cavity, or to the optical cavity's position control (usually a PZT mirror) for locking the optical cavity to the laser frequency. To which system we send the feedback signal is just a matter of choosing which system (laser 


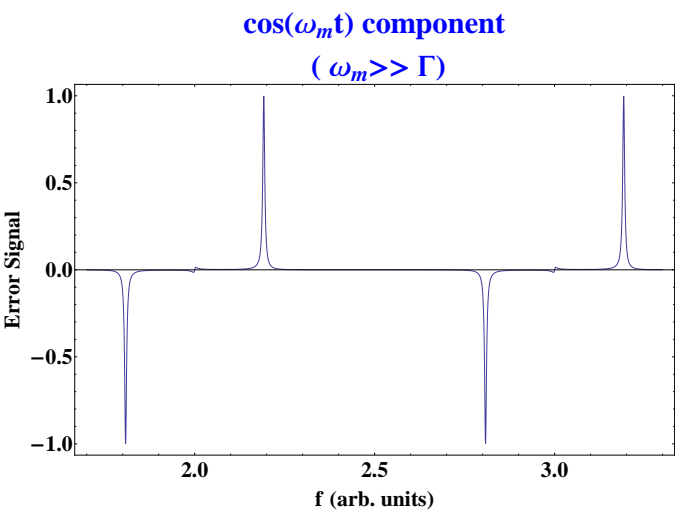

(a) The $\cos \omega_{m} t$ component when $\omega_{m} \gg \Gamma$

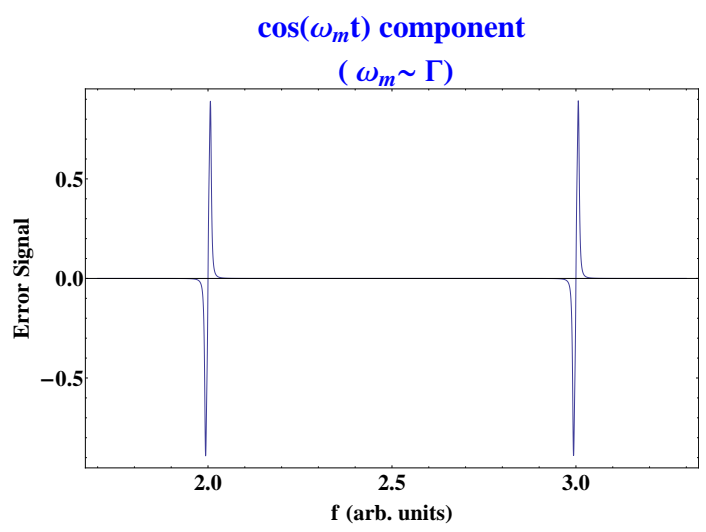

(c) The $\cos \omega_{m} t$ component when $\omega_{m} \sim \Gamma$

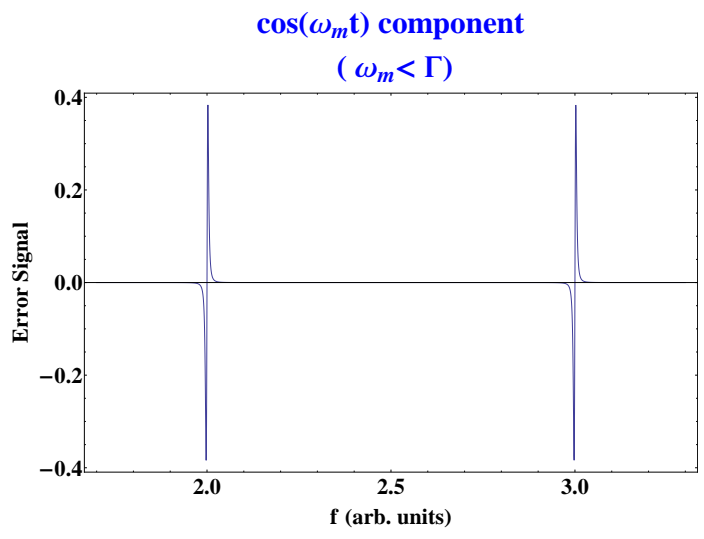

(e) The $\cos \omega_{m} t$ component when $\omega_{m}<\Gamma$

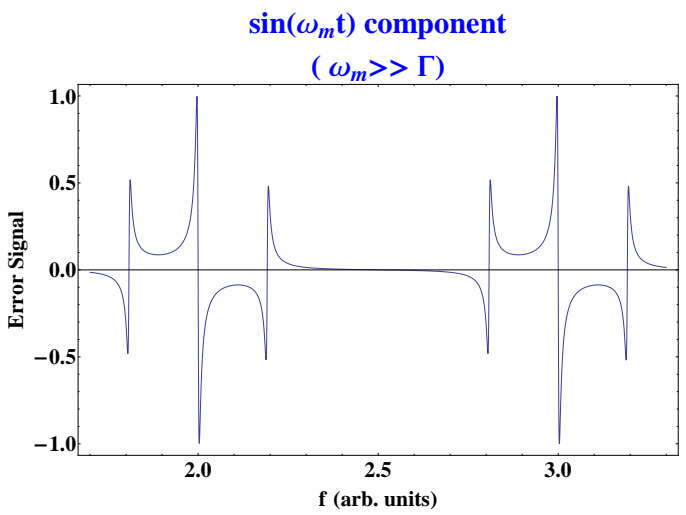

(b) The $\sin \omega_{m} t$ component when $\omega_{m} \gg \Gamma$

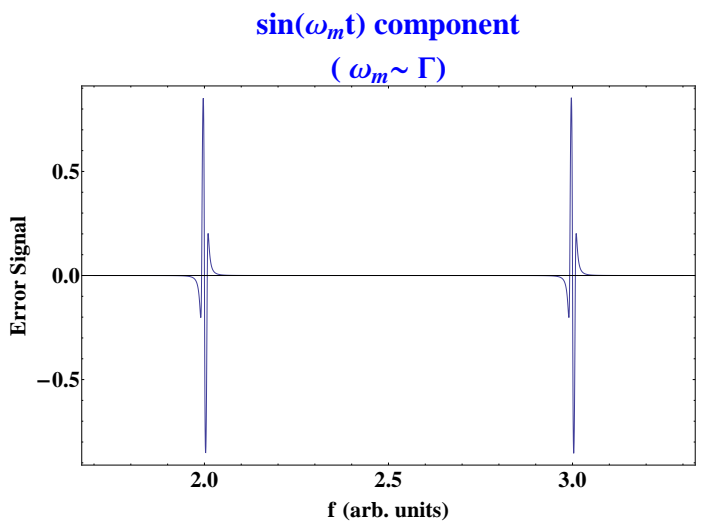

(d) The $\sin \omega_{m} t$ component when $\omega_{m} \sim \Gamma$

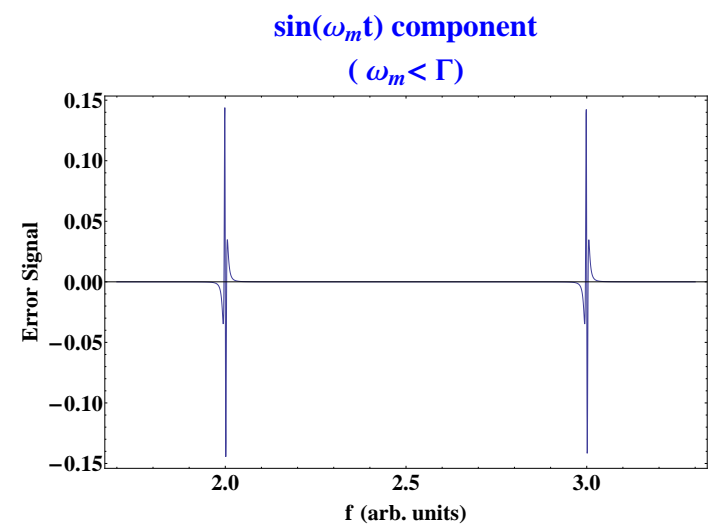

(f) The $\sin \omega_{m} t$ component when $\omega_{m}<\Gamma$

Figure 5.13: Different error signals after demodulation for different modulation frequencies. Fig. 5.13(a) and 5.13(b) are $\cos \omega_{m} t$ and $\sin \omega_{m} t$ components when the modulation frequency is a lot larger than the cavity linewidth $\left(\omega_{m}=30 \Gamma\right)$. Fig. $5.13(\mathrm{c})$ and $5.13(\mathrm{~d})$ are $\cos \omega_{m} t$ and $\sin \omega_{m} t$ components when the modulation frequency is about the same as the cavity linewidth $\left(\omega_{m}=1.1 \Gamma\right)$. Fig. 5.13(e) and $5.13(\mathrm{f})$ are $\cos \omega_{m} t$ and $\sin \omega_{m} t$ components when the modulation frequency is smaller than the cavity linewidth $\left(\omega_{m}=0.3 \Gamma\right)$. Plot parameters $r=0.99$, Finesses $=156.3$, $F S R=1$ and $\Gamma=0.0064 F S R$. 
or cavity) follows which, and the physics and principles for the rest are the same.

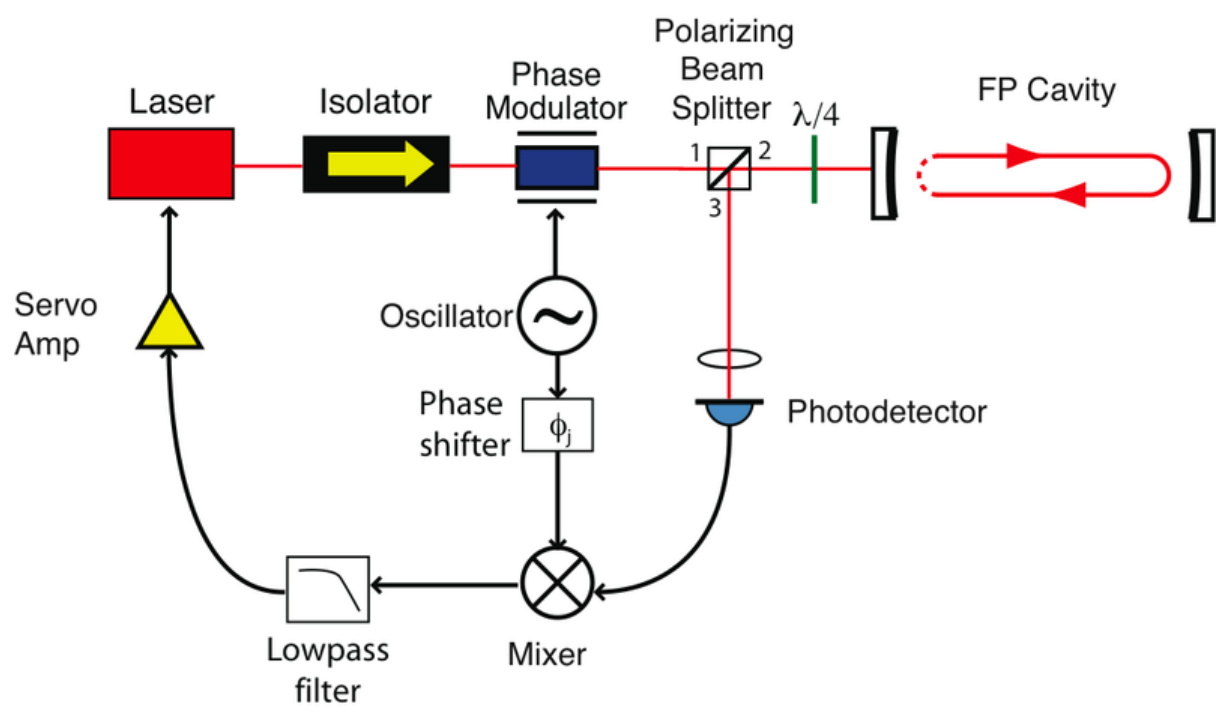

Figure 5.14: Schematic layout of a typical PDH servo loop to lock the frequency of a laser to a FabryPerot cavity. Light from the laser is sent through a phase-modulator and is then directed upon the cavity. The isolator is not involved in the PDH setup; it is present only to ensure that light from various optical components does not reflect back into the laser. Photodetector signal is demodulated (that is, passed through the mixer and the low-pass filter) to produce an error signal that is fed back into the laser's frequency control port. (from Wikipedia).

\section{Phase lock loop}

In our experiment we used two phase lock loops (PLL): one between the two frequencydoubled pump lasers so that their frequencies' difference is fixed to a certain value, the other between the local oscillator (LO) laser and one of the fundamental lasers in order to measure the squeezing nullifiers by locking the LO to a given "tooth" of the QOFC.

Figure 5.15 is a typical phase lock setup. We modematch two laser beams carefully at a beam splitter so that the beams overlap perfectly at a detector fast enough to see their beat note $\left|\omega_{1}-\omega_{2}\right|$. A signal generator is then used to mix a sine wave of 
freqnecy $\omega^{\prime}$ with the beat note, and the mixer's output is sent to a loop filter. The resulting correction signal is then fed back to one of the lasers so that we can lock the two lasers' frequencies to $\left|\omega_{1}-\omega_{2}\right|=\omega^{\prime}$.

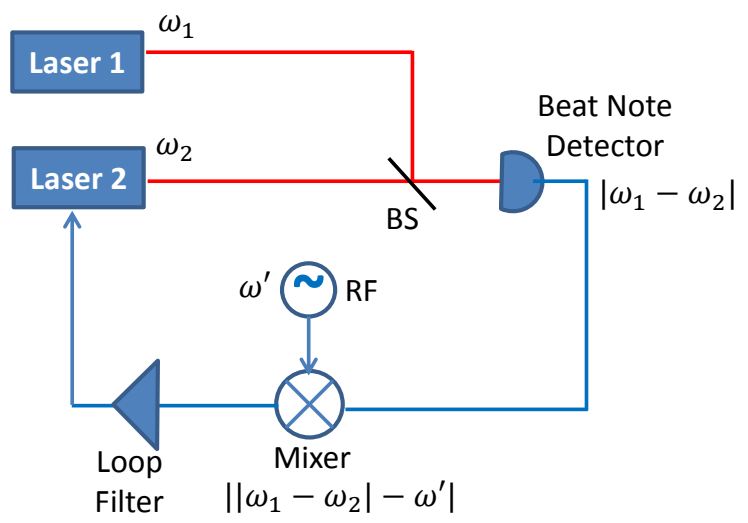

Figure 5.15: Typical phase lock loop between two lasers.

To put it in a mathematical form, we have the original laser signals $E_{1}(t)=$ $E_{1} \cos \omega_{1} t$ and $E_{2}(t)=E_{2} \cos \omega_{2} t$, and when they are combined, the total signal becomes the sum of them:

$$
\begin{aligned}
E_{3}(t) & =E_{1}(t)+E_{2}(t) \\
& =E_{1} \cos \omega_{1} t+E_{2} \cos \omega_{2} t
\end{aligned}
$$

And the detector's signal, which is the intensity of signal $E_{3}(t)$, is proportional to 
$E_{3}(t)^{2}$ :

$$
\begin{aligned}
& I_{3}(t) \propto E_{3}(t)^{2} \\
& =\left(E_{1} \cos \omega_{1} t+E_{2} \cos \omega_{2} t\right)^{2} \\
& =E_{1}^{2} \frac{1+\cos 2 \omega_{1} t}{2}+E_{2}^{2} \frac{1+\cos 2 \omega_{2} t}{2} \\
& \quad+E_{1} E_{2}\left[\cos \left(\omega_{1}+\omega_{2}\right) t+\cos \left(\omega_{1}-\omega_{2}\right) t\right]
\end{aligned}
$$

Due to the bandwidth limit of a detector, it can not be fast enough to see the high optical frequency terms, and only the beat note term $\cos \left(\omega_{1}-\omega_{2}\right) t$ can be detected, thus,

$$
I_{\text {det }} \propto E_{1} E_{2} \cos \left(\omega_{1}-\omega_{2}\right) t
$$

Send the detector's signal and the signal generator's RF signal $\cos \omega^{\prime} t$ into a mixer:

$$
\begin{aligned}
I_{\text {mixer }} & \propto I_{\text {det }} \times \cos \omega^{\prime} t \\
& \propto \frac{E_{1} E_{2}}{2}\left[\cos \left(\omega_{1}-\omega_{2}-\omega^{\prime}\right) t+\cos \left(\omega_{1}-\omega_{2}+\omega^{\prime}\right) t\right]
\end{aligned}
$$

The first term of Eq. (5.38) serves as the error signal (the second term will be filtered out after the low pass filter after the mixer). The error signal is oscillating but only every other slopes work, the other ones have the wrong sign. At the same time, the slop will go to zero when one gets closers to lock point.

Assuming $\omega_{1}>\omega_{2}$, and when the mixer's signal is locked (a non-oscillating flat line when the slope goes to zero), we have $\omega_{1}-\omega_{2}=\omega^{\prime}$, and this achieves phase lock between laser 1 and laser 2 so that their frequencies are $\omega^{\prime}$ away.

There are times that we need $\omega^{\prime}=0$, i.e., to lock two lasers at the exactly same frequency. For example, in our experiment we need the local oscillator laser to lock 
at the exact IR signal (half frequency) of the pump. To do this, we make use of an acousto-optical modulator (AOM) to first shift one frequency away by $\omega^{\prime}$, and then lock the newly-shifted signal with the second laser to $\omega^{\prime}$ so the original signal have the same frequency as the second laser, shown in Figure 5.16.

The mathematics are similar, and one thing to note is that in this case with AOM, after the mixer there are two oscillation frequencies: $\left|\omega_{1}-\omega_{2}-2 \omega^{\prime}\right|$ and $\left|\omega_{1}-\omega_{2}\right|$. Since we want to lock the two lasers to the same frequency, we want the second oscillation $\left|\omega_{1}-\omega_{2}\right|$ to disappear instead of the first one (which will result in $\left|\omega_{1}-\omega_{2}\right|=2 \omega^{\prime}$ ). This can be achieved by trial and error by verifying the beat note frequency or checking interference fringes of the two lasers.

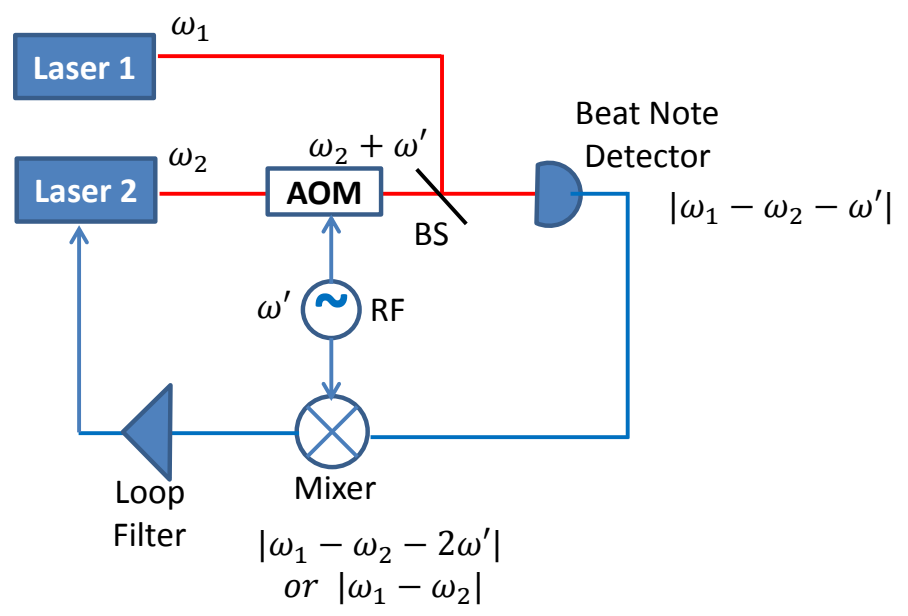

Figure 5.16: Phase lock loop between two lasers with AOM to lock at the same frequency.

\section{Balanced homodyne detection}

Balanced homodyne detection is used to measure the squeezed signal in our experiment. Ordinary photodetectors only detect light intensity or flux, homodyne detector, 
on the other hand, can detect the electric field quadrature operators. It is of great importance for the study of the phase sensitive quantum phenomena.

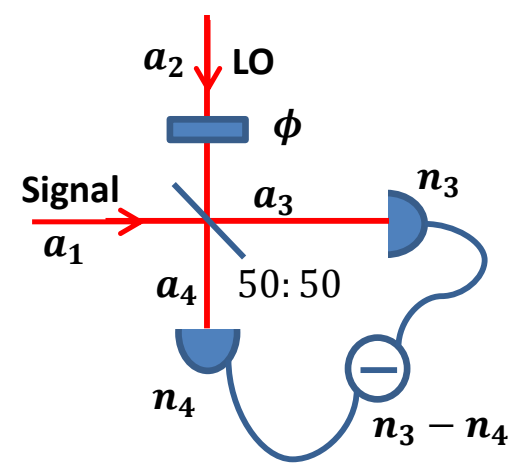

Figure 5.17: The balanced homodyne detector. The detector consists of a 50:50 beam splitter and two photodiodes connected together so that their photocurrents are subtracted. The signal field is incident at one of the input ports of the beam splitter, while the local oscillator (LO) is incident at the other.

Figure 5.17 shows the layout of the balanced homodyne detector. The detector consists of a 50:50 beam splitter and two photodiodes connected together so that their photocurrents are subtracted. The signal field is incident at one of the input ports of the beam splitter, while the local oscillator (LO) is incident at the other. In the following I will show briefly how it works.

We write the beam splitter matrix to be

$$
\left(\begin{array}{cc}
i r & t \\
t & i r
\end{array}\right)
$$

Notice that there are other ways/conventions to write the beam splitter matrix, and here I just choose a symmetric way. 
We can write out the output modes in terms of the input modes:

$$
\begin{aligned}
& \hat{a}_{3}=i r \hat{a}_{1}+t \hat{a}_{2} \\
& \hat{a}_{4}=t \hat{a}_{1}+i r \hat{a}_{2}
\end{aligned}
$$

The photo numbers at the detectors are:

$$
\begin{aligned}
n_{3} & =\hat{a}_{3}^{\dagger} \hat{a}_{3} \\
& =r^{2} \hat{a}_{1}^{\dagger} \hat{a}_{1}+t^{2} \hat{a}_{2}^{\dagger} \hat{a}_{2}+i r t\left(-\hat{a}_{1}^{\dagger} \hat{a}_{2}+\hat{a}_{1} \hat{a}_{2}^{\dagger}\right) \\
n_{4} & =\hat{a}_{4}^{\dagger} \hat{a}_{4} \\
& =t^{2} \hat{a}_{1}^{\dagger} \hat{a}_{1}+r^{2} \hat{a}_{2}^{\dagger} \hat{a}_{2}+\operatorname{irt}\left(\hat{a}_{1}^{\dagger} \hat{a}_{2}-\hat{a}_{1} \hat{a}_{2}^{\dagger}\right)
\end{aligned}
$$

Subtract the two detector's photon flux, and assume the quantum signal incident on input 1 and the local oscillator $\| \beta\left|e^{i \Phi}\right\rangle$ incident on input 2 (notice this is a 50:50 beam splitter so $\left.r=t=\frac{1}{\sqrt{2}}\right)$ :

$$
\begin{aligned}
n_{3}-n_{4} & =2 \operatorname{irt}\left(-\hat{a}_{1}^{\dagger} \hat{a}_{2}+\hat{a}_{1} \hat{a}_{2}^{\dagger}\right) \\
& =-|\beta|\left(\hat{a}_{1}^{\dagger} e^{i \Phi}-\hat{a}_{1} e^{-i \Phi}\right)
\end{aligned}
$$

Eq.(5.42) gives the quadrature operator of the signal field, and it is LO phase dependent. We can use this property to measure the quantum signal's quadrature operator by adjusting the LO's phase.

For ordinary homodyne detection (non-balanced homodyne detection), just set $t \gg r$ and only use $n_{4}$ (or $t \ll r$ if choose to use $n_{3}$ ), neglecting the small term $t^{2} \hat{a}_{1}^{\dagger} \hat{a}_{1}$ and the signal-independent term $r^{2} \hat{a}_{2}^{\dagger} \hat{a}_{2}$, then the measured signal will be proportional to the quantum field's quadrature as well. The balanced homodyne 
detection, however, is advantageous in that the classical noises are canceled during the substraction.

\section{Role of EOM phase in our nullifier selection}

In our experiment, the phase of the nullifier we measure is controlled by both the LO phase $\theta_{L O}$ and the EOM's modulation signal's phase $\theta_{0}$. We scan the PZT of the LO mirror to adjust the former and change the cable length of the EOM modulation signal generator to adjust the latter. This procedure is similar to that of our previous experiment (the 15 square cluster state in [35]).

After being phase modulated by the EOM and passed the filter cavity, the LO signal has two sidebands:

$$
\alpha_{m} \exp \left\{i\left[\omega_{0}+\left(n-\frac{1}{2} \Delta\right)\right] t+i \theta_{0}\right\}-\alpha_{m} \exp \left\{i\left[\omega_{0}-\left(n-\frac{1}{2} \Delta\right)\right] t-i \theta_{0}\right\}
$$

where $\Delta$ denotes the FSR of our OPO and $\theta_{0}$ is the EOM's modulation signal's phase at the sideband frequency $(n-1 / 2) \Delta$.

After reflection of the PZT, another phase term $\theta_{L O}$ adds to the expression:

$$
\begin{aligned}
& \alpha_{m} \exp \left(i \omega_{+} t+i \theta_{0}+i \theta_{L O}\right)-\alpha_{m} \exp \left(i \omega_{-} t-i \theta_{0}+i \theta_{L O}\right) \\
& =\alpha_{m} \exp \left(i \omega_{+} t+i \theta_{+}\right)-\alpha_{m} \exp \left(i \omega_{-} t+i \theta_{-}\right)
\end{aligned}
$$

where $\omega_{ \pm}=\omega_{0} \pm(n-1 / 2) \Delta$ and $\theta_{+}=\theta_{0}+\theta_{L O}, \theta_{-}=-\theta_{0}+\theta_{L O}$.

The 4-mode nullifiers centered at half frequency of $z$-pump we need to measure 
are (details in subsection 5.2.2):

$$
\begin{aligned}
& \left(Q_{+}^{(z)}-Q_{-}^{(z)}\right)+\left(Q_{+}^{(y)}-Q_{-}^{(y)}\right) \\
& \left(P_{+}^{(z)}+P_{-}^{(z)}\right)+\left(P_{+}^{(y)}+P_{-}^{(y)}\right)
\end{aligned}
$$

Using the generatlized quadrature $A(\theta)$ defined as

$$
A(\theta) \equiv a e^{-i \theta}+a e^{i \theta}
$$

Eq.(5.45) can be written as

$$
\left[A_{+}^{(z)}(\theta)-A_{-}^{(z)}(-\theta)\right]+\left[A_{+}^{(y)}(\theta)-A_{-}^{(y)}(-\theta)\right]
$$

The nullifiers centered at half frequency of $y$-pump are:

$$
\begin{aligned}
& \left(Q_{+}^{(z)}-Q_{-}^{(z)}\right)-\left(Q_{+}^{(y)}-Q_{-}^{(y)}\right) \\
& \left(P_{+}^{(z)}+P_{-}^{(z)}\right)-\left(P_{+}^{(y)}+P_{-}^{(y)}\right)
\end{aligned}
$$

Similarly they can be expressed by the generalized quadrature as:

$$
\left[A_{+}^{(z)}(\theta)-A_{-}^{(z)}(-\theta)\right]-\left[A_{+}^{(y)}(\theta)-A_{-}^{(y)}(-\theta)\right]
$$

In order to get the form $\left[A_{+}(\theta)-A_{-}(-\theta)\right]$ which is needed for both $y$ - and $z$ centered cases, compare it with Eq.(5.44), we need:

$$
\theta_{+}=-\theta_{-}+2 p \pi=\theta
$$

where $p$ is an integer.

Solve this and it requires $\theta_{L O}=p \pi$. As the LO phase $\theta_{L O}$ is being scanned 
during the experiment by a PZT, when it hits the integer of $\pi$ we obtain the nullifier term $\left[A_{+}(\theta)-A_{-}(-\theta)\right]$. More particularly, plug $\theta_{L O}=p \pi$ in, it terms out when when the EOM phase $\theta_{0}=p^{\prime} \pi$ we have the $Q$ quadrature and when the EOM phase $\theta_{0}=\left(p^{\prime}+\frac{1}{2}\right) \pi$ we have the $P$ quadrature. So to obtain the noncommuting quadratures the EOM phase $\theta_{0}$ needs adjusting $\pi / 2$ away, and in the experiment we change the EOM modulation signal's RF cable length to make this adjustment. However, it is essential to note that the squeezing should not be changed no matter what the EOM phase is and this is confirmed by our experimental observation also.

To get the nullifier forms of Eq.(5.49) and (5.50), we just need to add or subtract the two homodyne detectors' (measures $z$ - and $y$ - polarization respectively) signals using electronic circuits.

\section{Visibility measurement}

It is important to check the visibility between the local oscillator and the OPO seed beam, which indicates the optical path overlapping, phase lock between the two lasers, and the wavefront mode match are good. After careful alignments and improvement of phase lock performance, a visibility of about $99 \%$ is achieved.

Typical visibility figures in a good day are shown in Figure 5.18. 


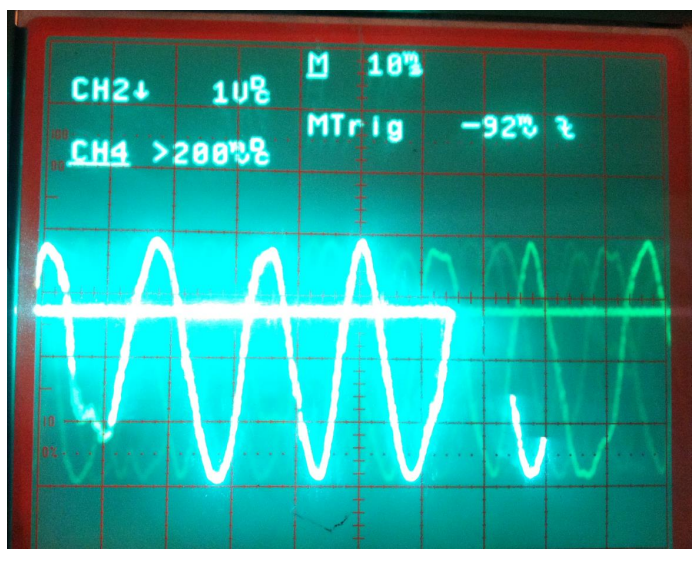

(a) Visibility figure

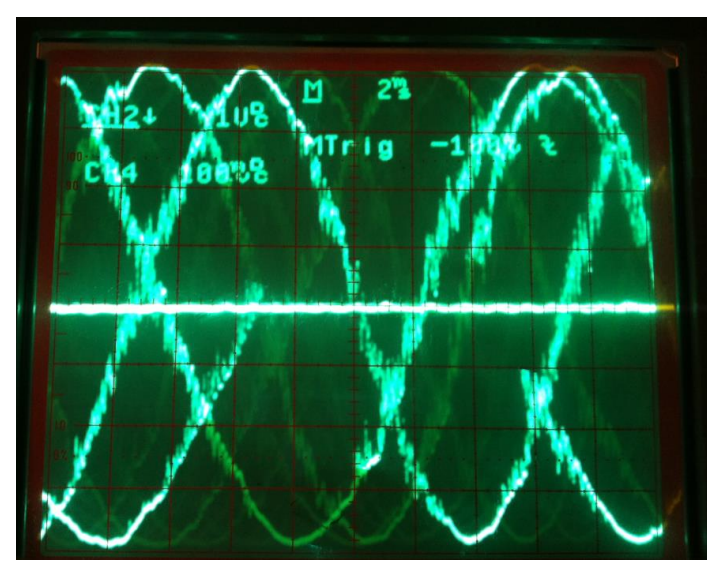

(b) Visibility figure

Figure 5.18: Typical visibility figures between the local oscillator and OPO seed beam. The PZT for local oscillator was scanned with a triangle signal at $5 \mathrm{~Hz}$ with a 5 Vpp amplitude. 


\subsubsection{Backward reflection}

\section{Back-reflection and measurement setup}

The OPO in the experiment has a four-mirror travelling wave cavity. However, during the experiment it was noticed that there was a backward reflection beam coming out of OPO.

The OPO has two crystals and two focusing waists: one crystal is a $1 \mathrm{~cm}$ PPKTP with $z z z$ quasi-phase match in a $30 \mu m$ focusing waist, and the other is a $1 \mathrm{~cm}$ yyy PPKTP crystal in a $130 \mu m$ waist.

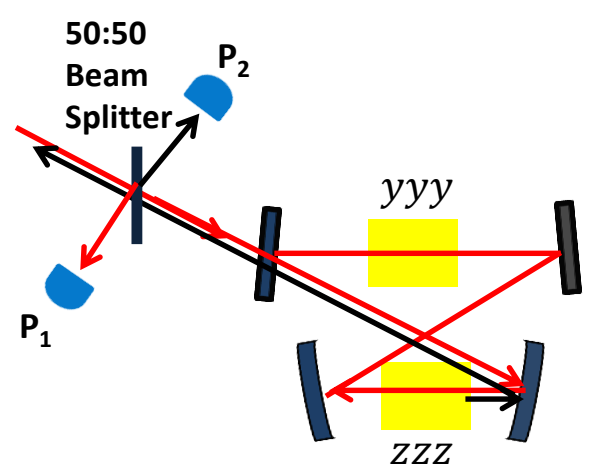

Figure 5.19: The experimental setup for measuring the back-reflection of OPO. The red lines indicate the forward direction beam path and the black lines indicates part of the back-reflection beam path. They should be overlapped in space and in this figure they are separated a little for the purpose of demonstration.

Because the back-reflection light travels back the same way as the input beam goes in, we made use of a beam splitter to monitor both the OPO input beam and the OPO back-reflected beam in real time, as shown in Figure 5.19. The input beam hit the 50:50 beam splitter so half of it reflected to the detector with power $P_{1}$ and half of it transmitted to the input to OPO, so the OPO input power is also $P_{1}$. Half of the back-reflection beam from the OPO was detected with power $P_{2}$, so the total 
power coming from the $\mathrm{OPO}$ is actually twice of that, i.e., $2 P_{2}$. So the back-reflection ratio is:

$$
R_{\text {back-reflection }}=\frac{2 P_{2}}{P_{1}}
$$

In the following we will study how the back-reflection ratio changes as we change different variables.

\section{Nonlinear effects?}

The first question we asked was, what was the cause of the back-reflection? As we have a nonlinear PPKTP crystal in the OPO one natural guess was that it could be from some nonlinear effects, such as counter propagating signal and idler in threeor four-wave mixing. We measured the change of back-reflection power $P_{2}$ versus the input power $P_{1}$, and found a linear dependence, as shown in Figure 5.20. The measurements were done when the OPO was on resonance.

This measurement can not eliminate the possibility of nonlinear effects instantly, because some nonlinear effects can have linear relation at certain situations. But at least this measurement doesn't give us any nonlinear dependence to indicate that this back-reflection was nonlinear-effects related. So we keep exploring other possible reasons. 


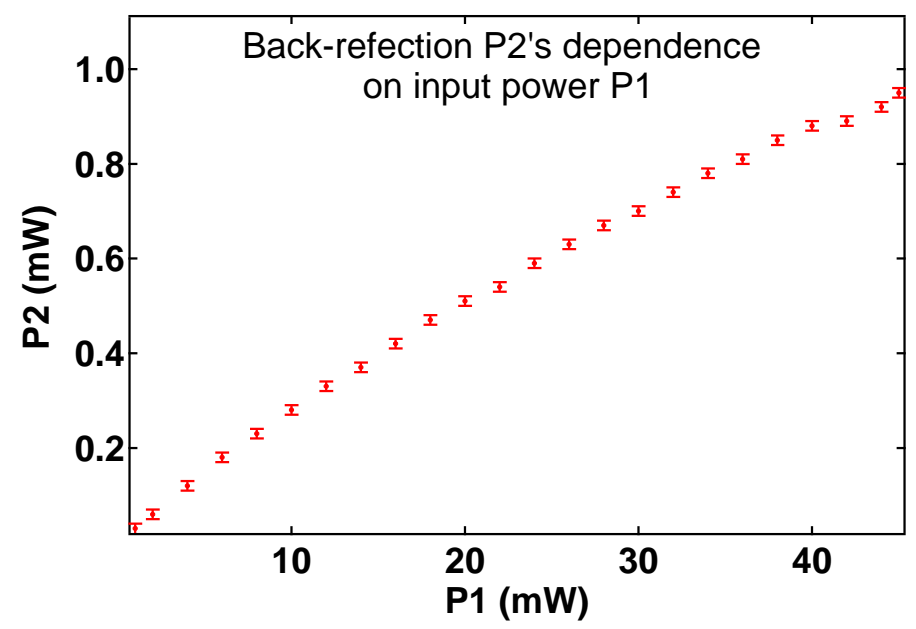

Figure 5.20: The back-reflection power $P_{2}$ 's dependence on the input power $P_{1}$. The relation looks linear. Here we focus on the trend only, and do not take into consideration the constant factors.

\section{Periodic oscillation as the temperature changes}

We continue to explore other aspects and variables. In the experiment we have two custom-made ovens to hold the two crystals respectively, so we can adjust each crystal's temperature independently. The ovens are temperature controlled by a feedback control loop through Peltier. When we changed the temperature of the PPKTP crystal, we observed a very noticeable change in the back-reflection power, as shown in Fig. 5.21. (However, when we chagned the temperature of the oven for the other KTP cyrstal, the back-reflection didn't oscillate.) So we went ahead to record the back-reflection power change as we tune the PPKTP crystal's temperature, shown in Fig. 5.21. Here the change of temperature is reflected by an electric DC read out from the thermistor and we will convert it to the corresponding temperature change later. 


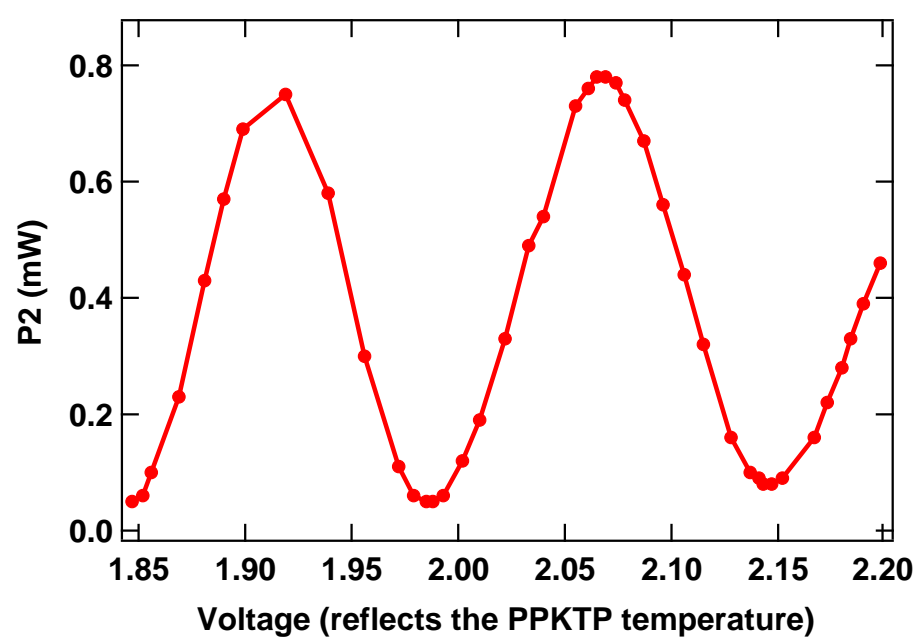

Figure 5.21: The back-reflection power $P_{2}$ 's dependence on the PPKTP's temperature, here reflected by the temperature controlling voltage. From this figure we can see clearly that the back-reflection oscillates as the temperature changes.

From Figure 5.21 we can see a clear oscillation pattern, which indicates some periodic behavior in the back-reflection power as the PPKTP crystal temperature changes. What could be the cause of this? It is probably not from any nonlinear effects as this has a clear oscillation pattern. Also, it is worth mentioning that when changing temperature in the other PPKTP crystal there is no oscillation behavior. So what are the reasons for the oscillation behavior in one crystal but not the other?

\section{Positive correlation between intracavity loss and the back-reflection}

Before we move on to find the reasons for the oscillation, there is one more interesting thing to point out: the intracavity loss changes the same way as the back-reflection. This confirms that the back-reflection is not only strange, but also affects the quality of the OPO.

We observed the intracavity loss (see subsection 4.2.3) and the back-reflection 
power at the same time as we tuned the PPKTP temperature change, as shown in Figure 5.22.

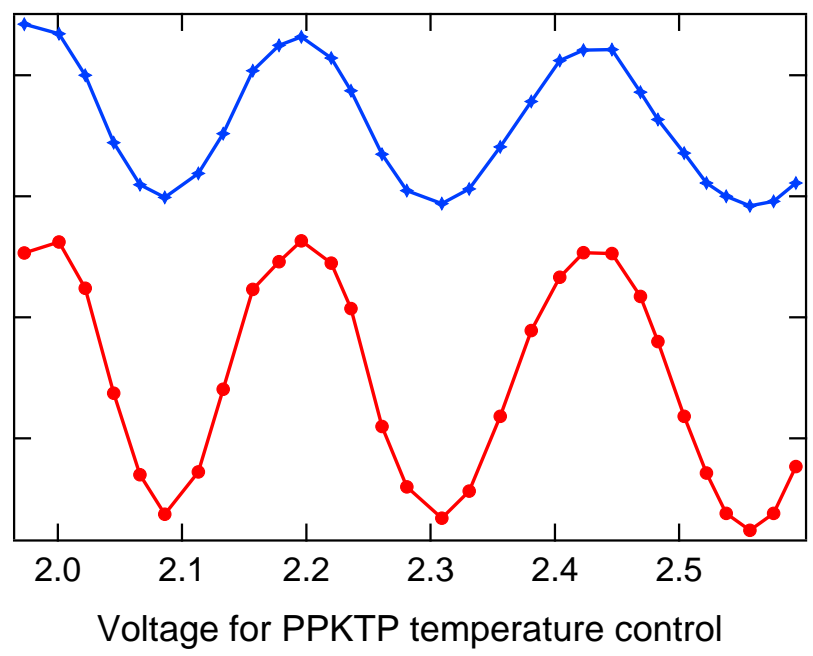

Figure 5.22: Simultaneous measurements for intracavity loss (blue trace) and the back-reflection (red trace). Positive correlation is clearly seen. The x-axis has arbitrary unit and no absolute values since here only the traces' changing trends are concerned.

A clear positive correlation is shown in the figure, which is reasonable because more back-reflection indicates more loss as it is not what the OPO is designed for or expected to behave. As we show later, as we managed to decrease the back-reflection power, the intracavity loss was decreased at the same time as well, resulting in a better quality OPO.

\section{Treat the PPKTP crystal as a cavity}

We all know that an optical cavity will have constructive or destructive interference depending on the relation between the wavelength and the cavity's length, resulting in a periodic oscillation in its reflection and transmission signal. In this section we will explore more about that possibility. 


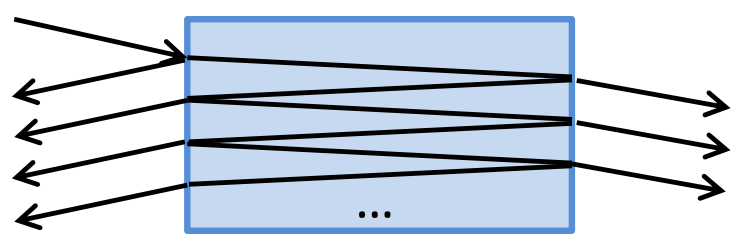

Figure 5.23: The reflection and transmission when a light beam hits a crystal.

Figure 5.23 shows the light hits the crystal and get reflected and transmitted when there is reflection from the crystal facets, and in the figure we exaggerated the incident angle to differentiate each beam from each round trips, and in reality they should overlap. When this happens, the crystal acts like an optical cavity. We can add the amplitudes of all the corresponding electromagnetic fields to calculate the total reflection and transmission fields:

$$
\begin{aligned}
E_{r}= & -r_{1} E_{0}+t_{1}^{2} r_{2} e^{i \delta} E_{0}+t_{1}^{2} r_{2} e^{i \delta} r_{1} r_{2} e^{i \delta}+\ldots \\
= & \frac{r_{2} e^{i \delta}-r_{1}}{1-r_{1} r_{2} e^{i \delta}} E_{0} \\
E_{t} & =t_{1} t_{2} e^{i \delta / 2} E_{0}+t_{1} t_{2} e^{i \delta / 2} r_{1} r_{2} e^{i \delta} E_{0}+\ldots \\
& =\frac{t_{1} t_{2} e^{i \delta / 2}}{1-r_{1} r_{2} e^{i \delta}} E_{0}
\end{aligned}
$$

where $r_{1}, r_{2}$ are the reflection coefficients of the front and back facet of the crystal, and $t_{1}, t_{2}$ are the transmission coefficients of the front and back facet. $\delta$ is the optical phase from the round trip in the crystal, $\delta=2 n L \frac{\omega}{c}$, where $L$ is the length of the crystal.

The total reflection (transmission) coefficients of the crystal is the ratio of the 
reflection (transmission) amplitude over the incident amplitude $E_{0}$ :

$$
\begin{aligned}
& r_{\text {crystal }}=\frac{r_{2} e^{i \delta}-r_{1}}{1-r_{1} r_{2} e^{i \delta}} \\
& t_{\text {crystal }}=\frac{t_{1} t_{2} e^{i \delta / 2}}{1-r_{1} r_{2} e^{i \delta}}
\end{aligned}
$$

Due to energy conservation, $|r|^{2}+|t|^{2}=1$. The following figure shows $|r|^{2}$ and $|t|^{2}$ change as the crystal's index of refraction change:

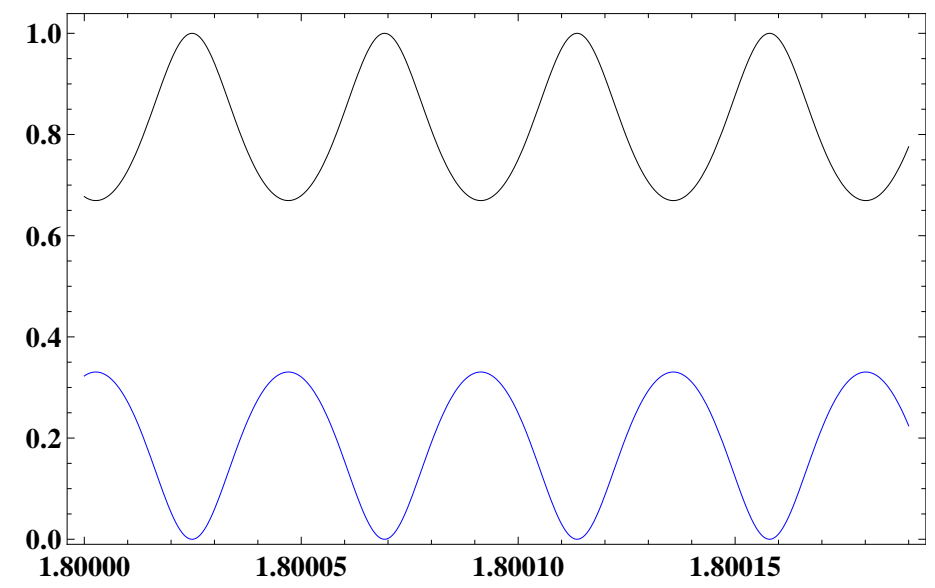

Figure 5.24: The reflectivity (lower trace) and transitivity (higher trace) of a crystal as index of refraction $n$ changes. Here $r_{1}^{2}=r_{2}^{2}=0.1, L=1.2 \mathrm{~cm}$.

As we can see in Fig. 5.24, as the index of refraction $n$ changes, the reflectivity and transitivity oscillate. This makes sense as we change $n$, the optical path of the crystal changes, and the round trip phase $\delta$ changes too. Once the change is $2 \pi$, an oscillating period is complete. For Fig. 5.24 and 5.21 there are both oscillating patterns, and the study about the connections between their oscillation periods will be shown in the following section. 


\section{A tentative model for the whole picture}

From the previous sections' discoveries and calculations, we have strong reason to believe that when light enters our OPO, it can get coupled with the "small cavity" formed by the PPKTP crystal, and then some light will get reflected back, changing from forward propagating to counter propagating. As we change the crystal's temperature, the reflection property of the "small cavity" oscillates due to the change of the index of refraction $n$, and this in term causes the total back-reflection coming from the OPO also to oscillate. The other KTP crystal isn't involved in this coupling because as we change its temperature, no oscillation behavior was observed.

So here the PPKTP crystal serves as a "coupling cavity", to couple the "forward propagating" mode with the "backward propagating" mode. It works like this: if the PPKTP crystal didn't reflect any incoming light, the light would always propagate along the initial direction (let us call this direction the "forward" one); and once the PPKTP reflects the light, it will start propagating the other way - "backward", thus we can see some back-reflection light; and if the PPKTP reflects the backward propagating light, it will change back to the forward propagating mode, and so on and so forth. So the PPKTP crystal couples the "forward propagating" and "backward propagating" modes and is like a bridge to connect and switch them, as shown in Figure 5.25.

When we calculate the total back-reflection from the coupling cavity theory, we use an equivalent system model, as shown in Figure 5.26. Here we draw the two 


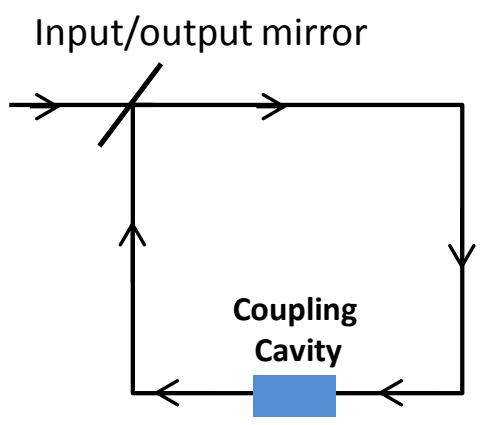

Forward Propagating

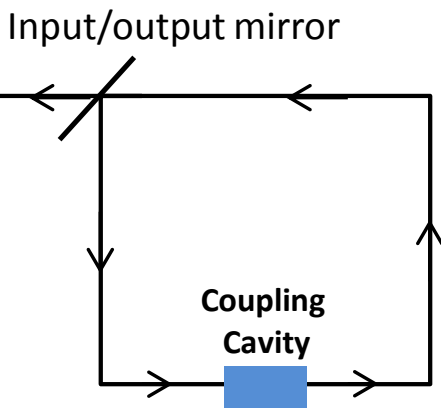

coupled

Backward Propagating

Figure 5.25: The "forward propagating" mode and the "backward propagating" mode coupled by the cavity formed by the PPKTP crystal.

propagating mode as two cavities that have the same cavity length $d$, connecting by the crystal cavity (length $L$ ). The left-hand-side cavity represents the forward propagating mode, and the right-hand-side cavity represents the backward propagating mode; if the light is trapped in the left-hand-side cavity, that means that it is in the forward propagating mode, and when it goes through the coupling cavity to reach the right-hand-side cavity, that means that it is in the backward propagating mode. Since the backward propagating light leaking through the output mirror forms the back-reflection light we observed, the transmission through the right-most mirror $r_{3}$ gives us the back-reflection power we observe. The difference between Figures 5.25 and 5.26 is that, in Figure 5.25 the light needs to reflect on the coupling cavity in order to switch to the other mode, while in Figure 5.26 the light needs to transmit the coupling cavity in order to switch to the other mode, and this can be easily taken care of during the calculation by exchanging the reflection and transmission coefficient (Eq.(5.54) and (5.55) of the coupling cavity. 


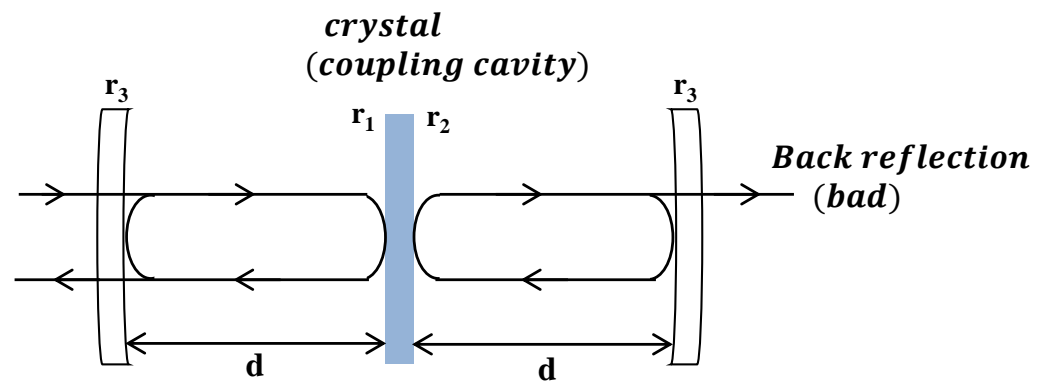

Figure 5.26: The model for the system where the PPKTP crystal serves as the coupling cavity to couple the forward and backward prorogating modes. The transmission through the right-most mirror $r_{3}$ is equivalent to the back-reflection we observe. In Fig. 5.25 (the ring cavity model), the crystal's reflection creates the coupling; while in this figure (standing wave cavity model), it is the crystal's transmission that does that.

Now with the help of Figure 5.26, we can calculate the back-reflection ratio. We first find out the reflection and transmission coefficients for the coupling cavity (the crystal), and then treat it as a virtual mirror to calculate the reflection and transmission coefficients for the new cavity formed by the first mirror (the left $r_{3}$ mirror) and the crystal cavity, and then we treat this new cavity we just calculated as a virtual mirror, and again adds in the third mirror (the right $r_{3}$ mirror) to calculate the total reflection and transmission of the whole system.

As shown in Eq. (5.54) and (5.55), we already found the reflection and transmission for the crystal cavity, but here we switch the reflection and transmission as discussed earlier. So for the coupling cavity, we have,

$$
\tilde{t}=r_{\text {crystal }}=\frac{r_{2} e^{i \delta}-r_{1}}{1-r_{1} r_{2} e^{i \delta}}
$$




$$
\tilde{r}=t_{\text {crystal }}=\frac{t_{1} t_{2} e^{i \delta / 2}}{1-r_{1} r_{2} e^{i \delta}}
$$

Then treat this coupling cavity as a virtual mirror with reflection coefficient $|\tilde{r}|$ and transmission coefficient $|\tilde{t}|$ (here the absolute value is used to avoid the phase confusion caused by switching the reflection and transmission earlier), and a new cavity formed by it and the first mirror (the left most mirror) has coefficients:

$$
\begin{aligned}
& r_{12}=\frac{|\tilde{r}| e^{i \delta_{0}}-r_{3}}{1-|\tilde{r}| r_{3} e^{i \delta_{0}}} \\
& t_{12}=\frac{|\tilde{t}| t_{3} e^{i \delta_{0} / 2}}{1-|\tilde{r}| r_{3} e^{i \delta_{0}}}
\end{aligned}
$$

where $\delta_{0}=2 d \frac{\omega}{c}$ is the optical phase for a round trip for the new cavity with length d. Again treat this new cavity as a virtual mirror with reflection coefficient $r_{12}$ and transmission coefficient $t_{12}$, and the whole system formed by it and the last mirror (the right $r_{3}$ mirror) has coefficients:

$$
\begin{aligned}
r_{\text {total }} & =\frac{r_{3} e^{i \delta_{0}}-r_{12}}{1-r_{12} r_{3} e^{i \delta_{0}}} \\
t_{\text {total }} & =\frac{t_{12} t_{3} e^{i \delta_{0} / 2}}{1-r_{12} r_{3} e^{i \delta_{0}}}
\end{aligned}
$$

So the back-reflection ratio we observe experimentally is:

$$
R_{\text {Back-reflction }}=\left|t_{\text {total }}\right|^{2}=\left|\frac{t_{12} t_{3} e^{i \delta_{0} / 2}}{1-r_{12} r_{3} e^{i \delta_{0}}}\right|^{2}
$$


A simulation for Eq. (5.62) is shown as in Fig. 5.27.

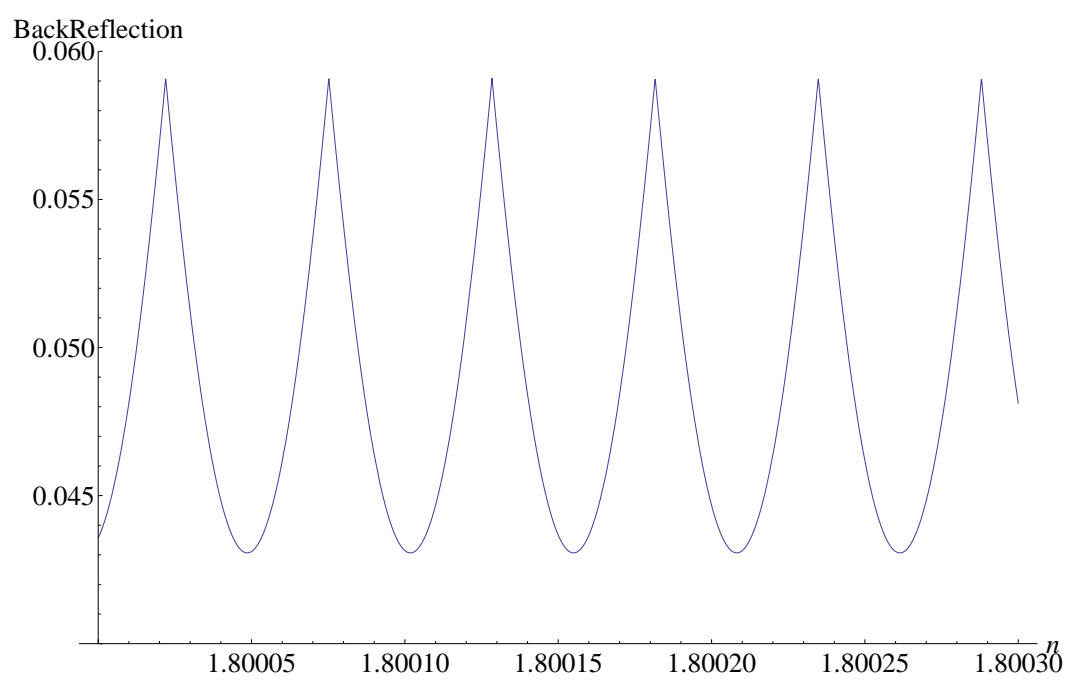

Figure 5.27: Coupling cavity model simulation for back reflection R. The $x$ axis is the PPKTP's index of refraction n, and the $y$ axis is the back reflection ratio. Simulation parameters $r 1=r 2=0.01, r 3=0.97$, PPKTP crystal length $L=1 \mathrm{~cm}$.

From Fig. 5.27 we can see that the period for the oscillation is $\Delta n=5.26 \times 10^{-5}$, and given the KTP crystal's thermal parameter [70] $\frac{d n}{d T}=0.000016$, the corresponding period in temperature is $\Delta T=\frac{\Delta n}{d n / d T}=3.3^{\circ}$.

In the experiment, the average change of temperature in one period is about $0.24 \mathrm{~V}$, and this corresponds to $2.6^{\circ}$ temperature change based on the thermistor parameters. This agrees reasonably well with the simulation result and they are in the same order of magnitude. The difference might be due to the inaccuracy of the voltage reading, the crystal length not being exactly $1 \mathrm{~cm}$, the inaccuracy of various parameters, etc. In the simulation of coupling cavity model Fig. 5.27, we can see that the back reflection is about $5 \%$, agreeing well with the experiment measurement also. 


\section{Back-reflection problem solved}

Knowing that the source of the back-reflection problem is from the lighting being coupled into the crystal, we hope to "uncouple" the light. A simple way to do this is to tilt the crystal a little bit so that the light is not mode-matched to the crystal cavity anymore, thus kill the coupled cavity effect (of course, the facet of the crystal can still reflect once, but without the small cavity enhancement, the reflection is going to be very small, plus the reflected light will not be the mode matched to the OPO cavity so it can't be amplified to a decent amount).

We did try tilting the PPKTP crystal little by little by "walking" adjustment: tilt the crystal, re-modematch the seed beam, and measure the back reflection power $P_{2}$ to see whether it is decreased, otherwise switch to the other direction. Once we found the right way to go, the back-reflection power decrease very quickly, along with the intracavity loss. Merely by tilting the crystal a little bit, we were able to decrease the back-reflection ratio from more than $6 \%$ to less than $0.2 \%$, and decrease the intracavity loss from $1.1 \%$ to $0.3 \%$. 


\subsection{Experimental results}

To verify the dual-rail wire structure, the 4-mode nullifiers with the form in Eq.(5.15) to (5.18) were measured with Homodyne detection. One example of what we measured is shown in Figure 5.28. In the following I will show the experimental results.

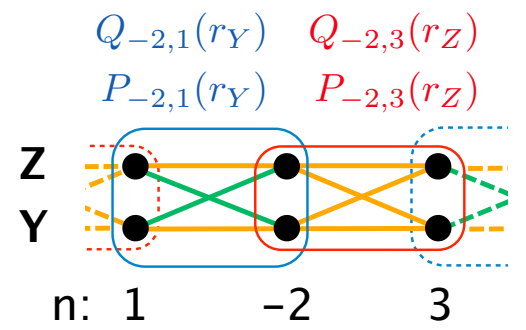

Figure 5.28: Visualization of the experimentally measured nullifiers of Eq.(5.15)(5.18) (blue and red boxes) on the dual-rail graph state. As shown in the text, simultaneous squeezing of $Q_{-2,3}\left(r_{z}\right)$ and $Q_{-2,1}\left(r_{y}\right)$ is equivalent to squeezing of the canonical nullifiers of Eq.(5.19) and (5.20).

\subsubsection{One-wire case}

First I will show the results for the one wire case, i.e., when $m=1$ for $\left|p_{y}-p_{z}\right|=$ $2 m$. The Figure 5.28 only shows one unit group in the wire, and we extended the measurement to the whole wire (as far as our EOM frequency can reach) to verify the whole structure and showed that the entanglement are the same wherever we measure along the wire. Here I first present a qualitative summary of the results.

Figure 5.29 displays typical squeezing signals that evidence the graph structure. It proves a "unit cell" of the graph, i.e., which verifies Eq.(5.19) and (5.20) for $n=1$ and $m=1$. The uncorrected squeezing level was $-3.2 \pm 0.2 \mathrm{~dB}$ throughout our measurements. Deconvolving the "dark" electronic noise floor of -96 dBm, $-13 \mathrm{~dB}$ 

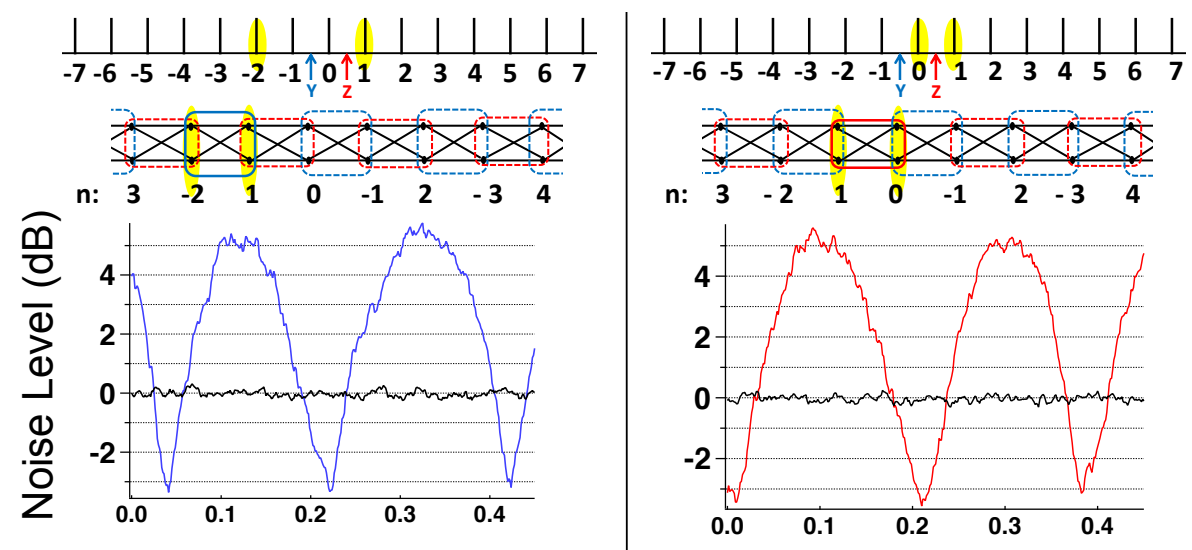

Figure 5.29: Zero-span spectrum analyzer traces of raw squeezing measurements for $m=1$ quantum wire. The QOFC is at the top, with the pump half-frequencies denoted by the blue and red arrows, and the quantum graph is beneath it. The highlighted modes indicate the LO sidebands. The black traces indicate the vacuum noise level (horizontal axis is the scanning LO phase). Center frequency: $1.25 \mathrm{MHz}$. Resolution bandwidth: $30 \mathrm{kHz}$. Video bandwidth: $30 \mathrm{~Hz}$.

from the vacuum noise level (the LO power was $2 \mathrm{~mW}$ at each photodiode), yielded an actual squeezing level of $-3.4 \pm 0.2 \mathrm{~dB}$, enough to satisfy the van Loock-Furusawa inseparability criterion level of $-3 \mathrm{~dB}$.

One important check we did was to see whether there was still squeezing among unconnected modes, i.e., to check incorrect graph nodes, exemplified by Figure 5.30. The LO was phaselocked at an offset from half the frequency of one pump, which allowed us to measure nullifier observables over the "wrong" modes. From the quantum wire graph it is clear that frequency 1 and 2 are not connected, so when we measured the quadratures of these modes only phase-independent excess quantum noise was observed, in good agreement with theoretical predictions, proving that the measured observable is not a nullifier in this case. The complete set of such checks is prohibitively large but all of the ones we tested gave the predicted negative result. 
All of these measurements demonstrate that a 60-mode dual-rail cluster state was generated in the QOFC.
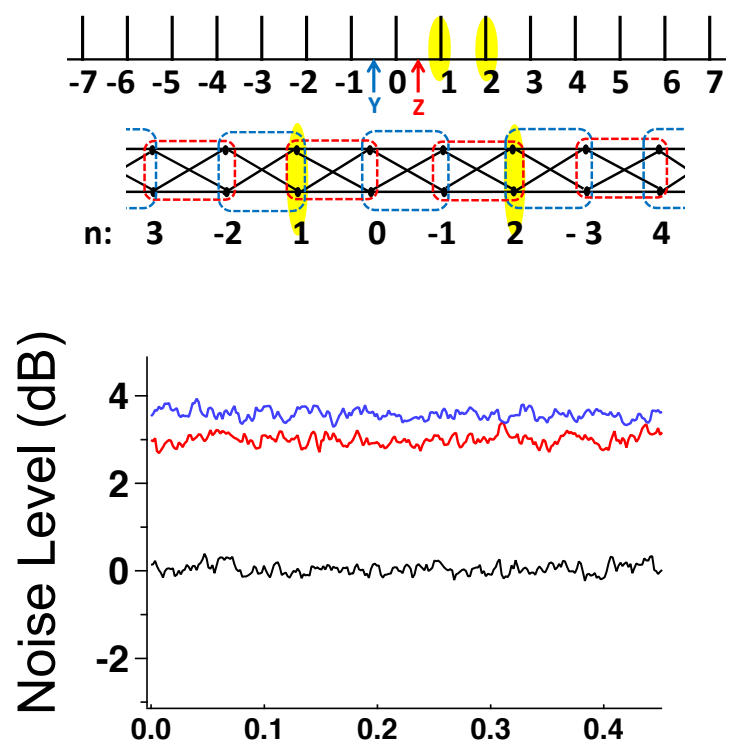

Figure 5.30: "Wrong" modes checks for one-wire case. Only excess quantum noise was detected, no squeezing observed, as expected. Figure legends same as in Figure 5.29 .

We have measured and confirmed the dual-rail wire structure up to 60 modes for one-wire case. We change the local oscillator's frequency combined with its sidebands modulated by EOM to precisely pinpoint which modes we are measuring. The y pump centered 4-mode nullifier (Eq. 5.17 and 5.18) measurements are shown in Figure 5.31, and the z pump centered 4-mode nullifier (Eq. 5.15 and 5.16) measurements are shown in Figure 5.32. These are all original measurements without any noise correction; after the homodyne detector's electronic noise correction the squeezing will increase $0.2 \mathrm{~dB}$ more for all the cases. The total number of modes we measured in one wire is 60 , which is only limited by the EOM measurement ability (while the actual number of 
modes in our wire should be many orders of magnitude more). A complete squeezing data for one wire case are showing in Figure 5.31 and 5.32 .

We set the local oscillator frequency to measure the modes that are not supposed to have connections for some wrong-frequency checks, and it shows that when we are not measuring the right modes (intentionally tuning the local oscillator frequency to other non-pump-symmetric modes), although we are measuring the same nullifiers form, we do not obtain squeezing. It shows that there is only antisqueezing, and the antisqueezing levels are independent of the local oscillator's phase, which agrees with our results, shown in Figure 5.33. 


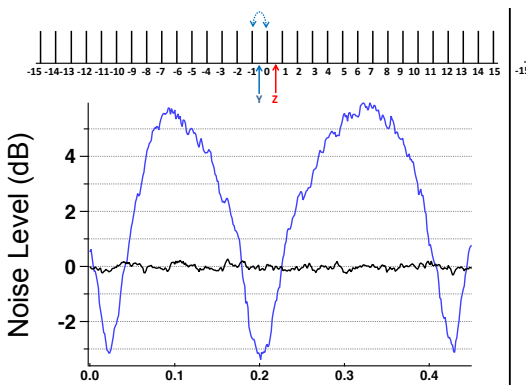

(a) y-pump-centered 0.5 FSR

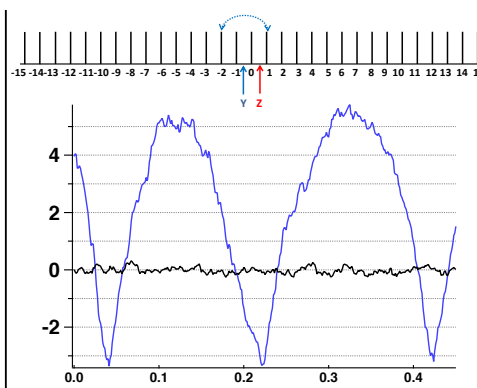

(b) y-pump-centered 1.5 FSR

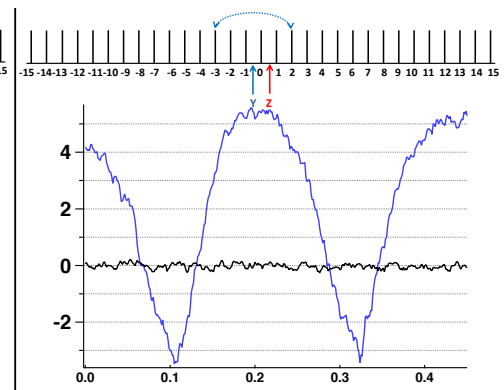

(c) y-pump-centered 2.5 FSR

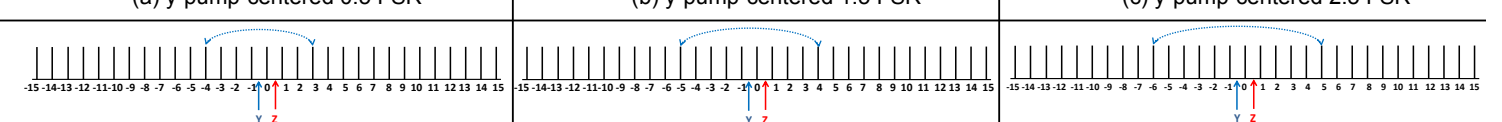

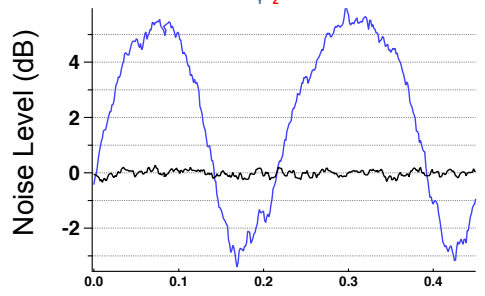

(d) y-pump-centered 3.5 FSR
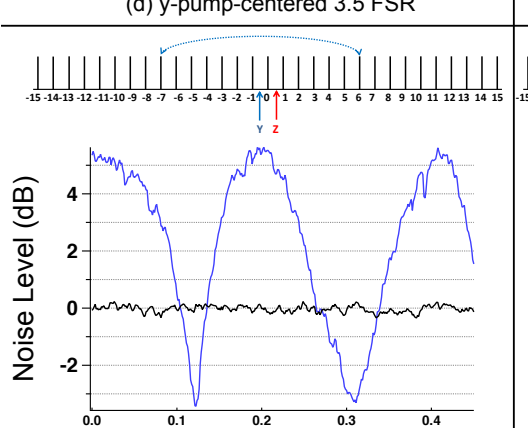

(g) y-pump-centered 6.5 FSR

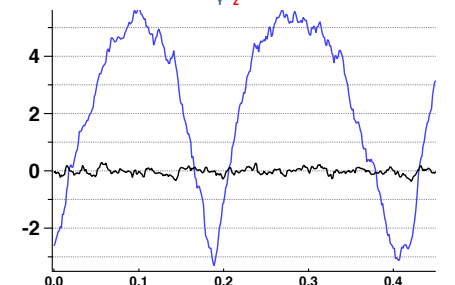

(e) y-pump-centered 4.5 FSR

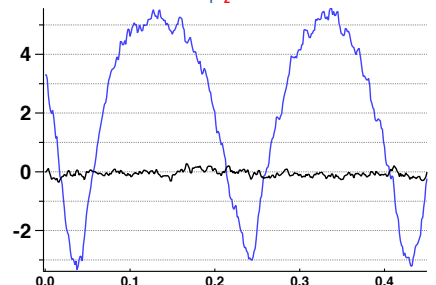

(f) y-pump-centered 5.5 FSR
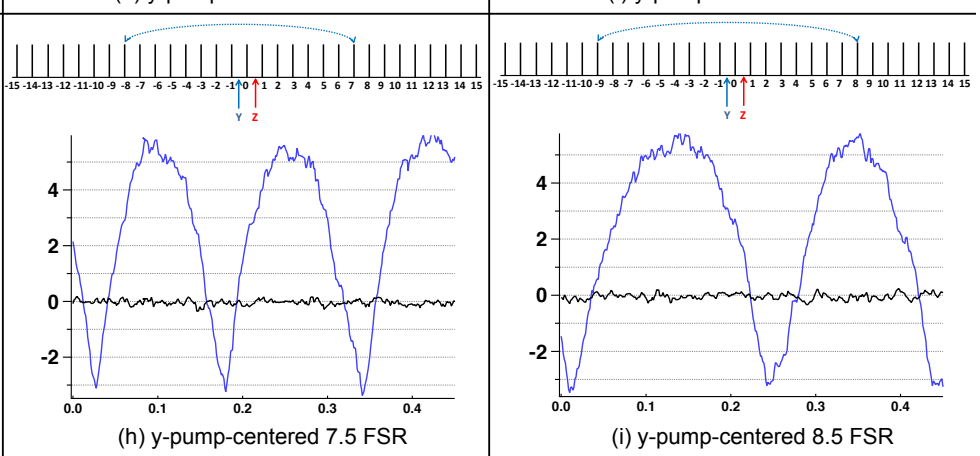

(i) y-pump-centered 8.5 FSR
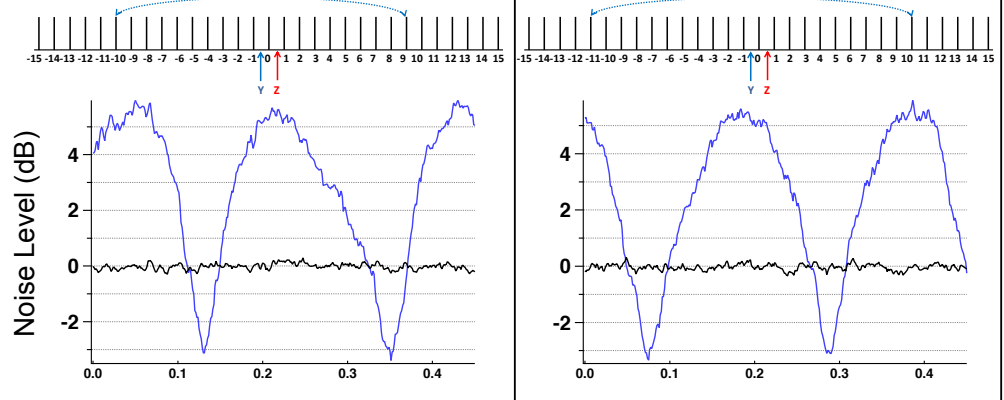

(j) y-pump-centered 9.5 FSR

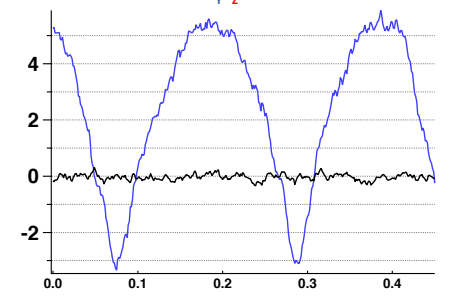

(k) y-pump-centered 10.5 FSR

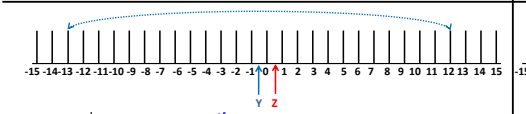

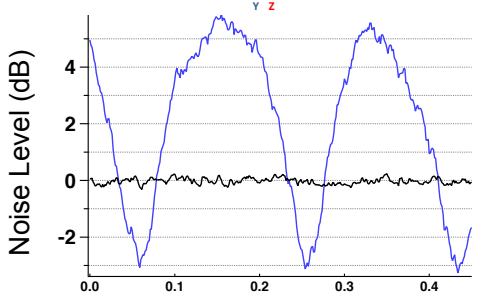

(m) y-pump-centered 12.5 FSR

Local Oscillator Phase (arb. unit)

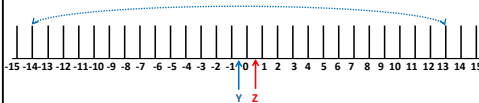

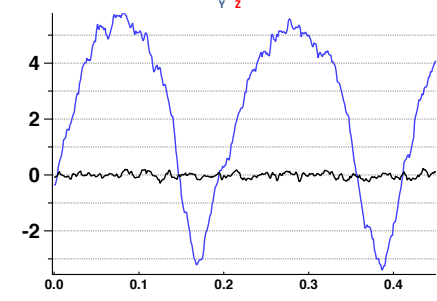

(n) y-pump-centered 13.5 FSR Local Oscillator Phase (arb. unit)

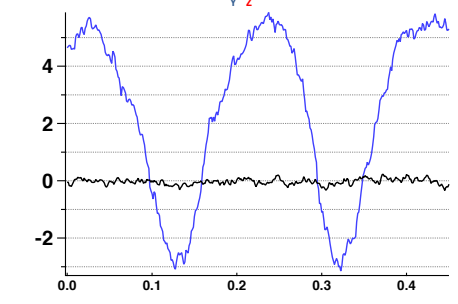

(I) y-pump-centered 11.5 FSR
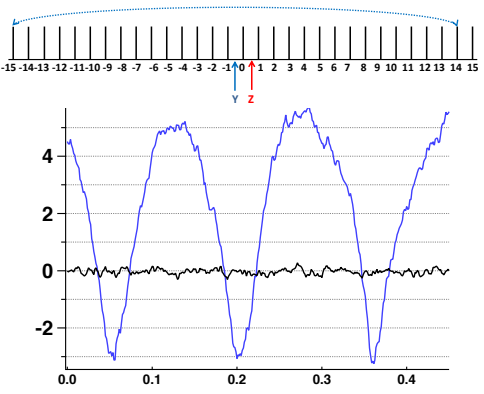

(o) y-pump-centered 14.5 FSR Local Oscillator Phase (arb. unit)

Figure 5.31: One-wire case y pump centered nullifiers squeezing measurements. 


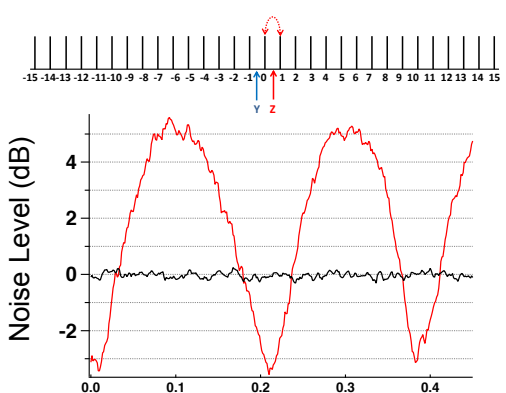

(a) z-pump-centered 0.5 FSR

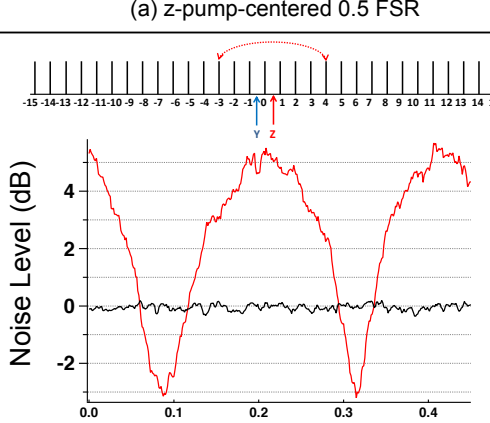

(d) z-pump-centered 3.5 FSR
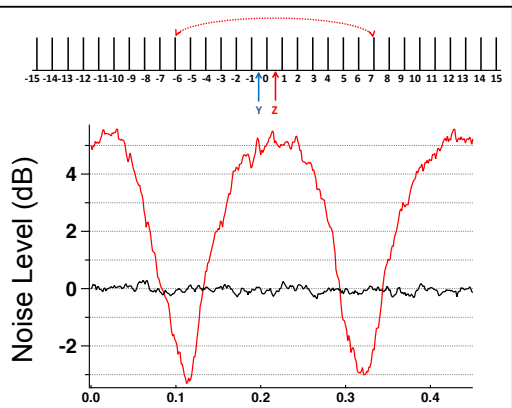

(g) z-pump-centered 6.5 FSR

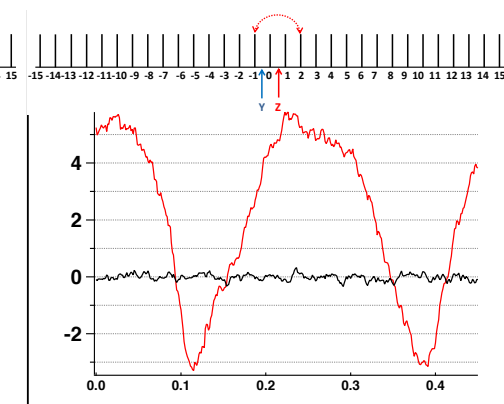

(b) z-pump-centered 1.5 FSR
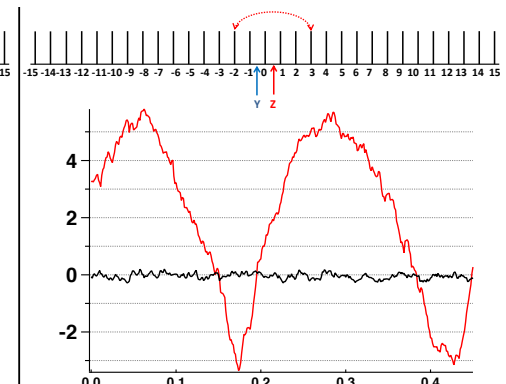

(c) z-pump-centered $2.5 \mathrm{FSR}$

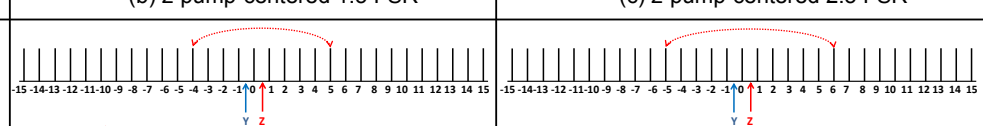

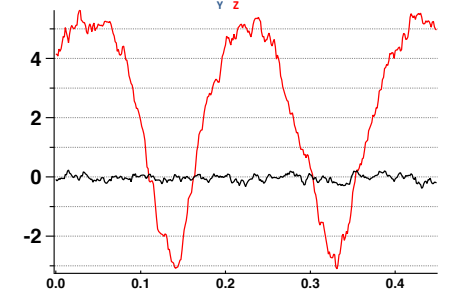

(e) z-pump-centered 4.5 FSR

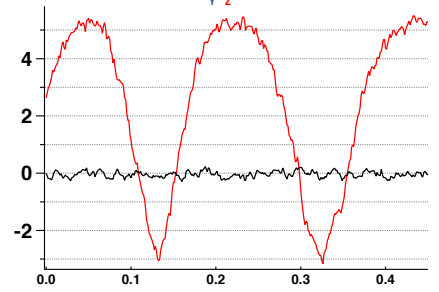

(f) z-pump-centered 5.5 FSR

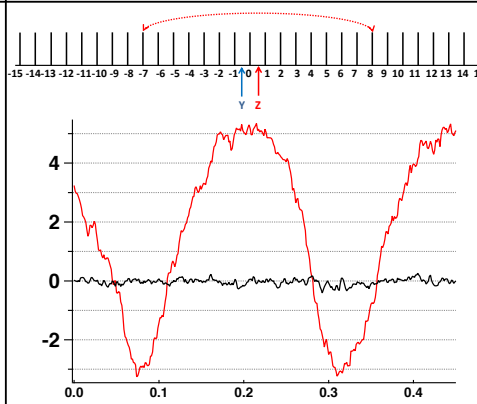

(h) z-pump-centered 7.5 FSR
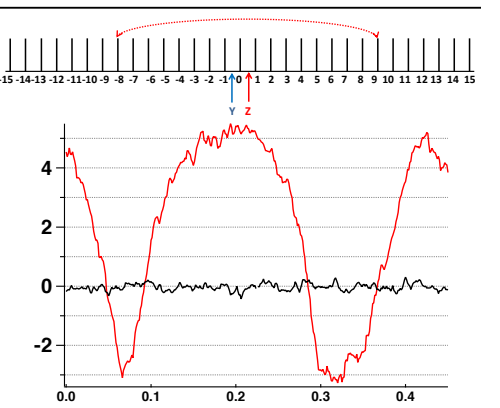

(i) z-pump-centered 8.5 FSR
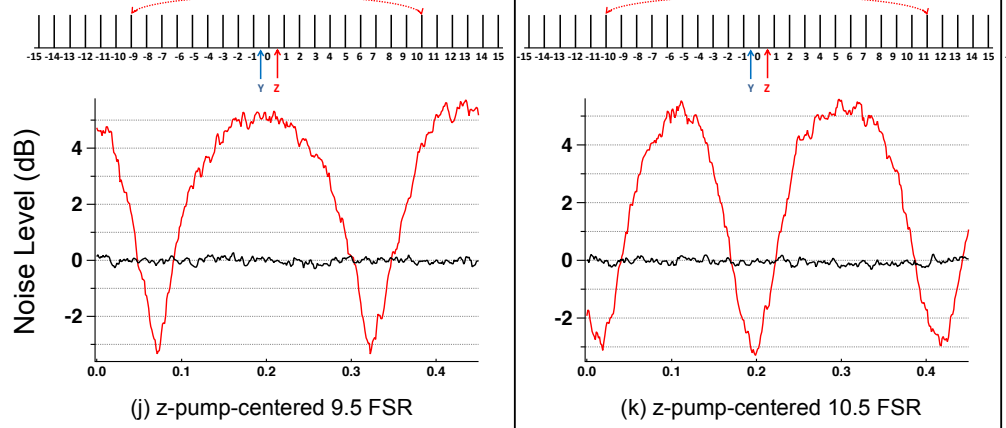

(k) z-pump-centered 10.5 FSR
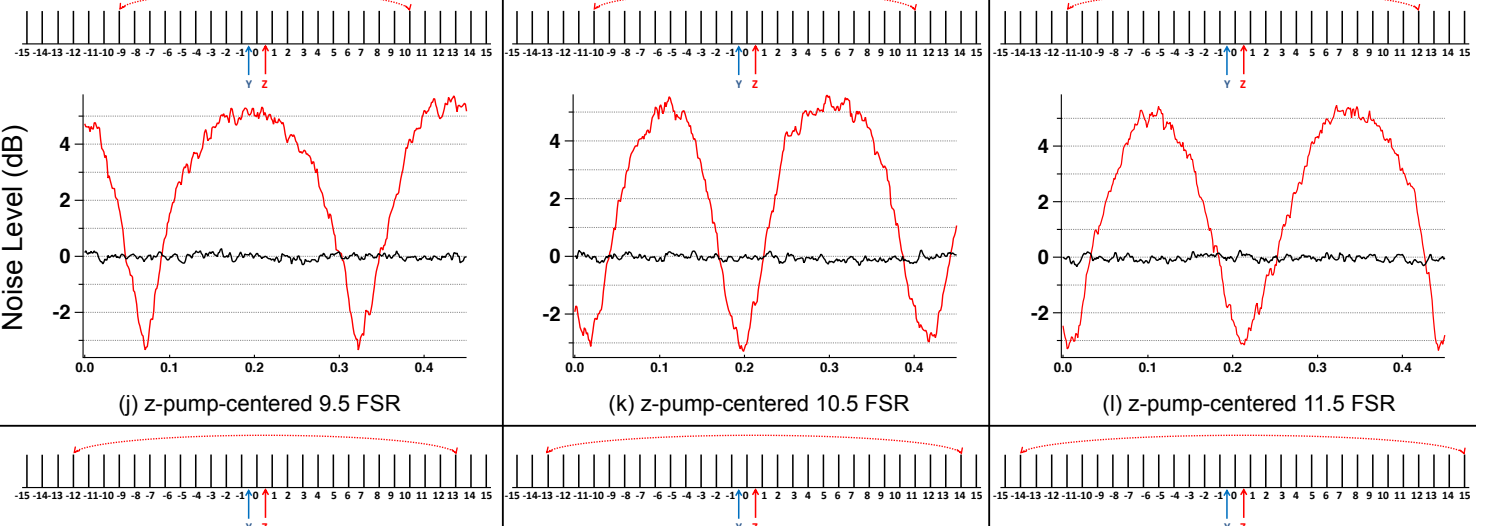

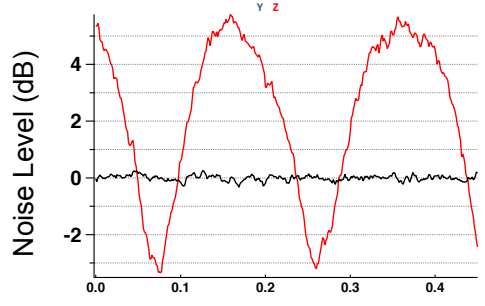

(m) z-pump-centered 12.5 FSR

Local Oscillator Phase (arb. unit)

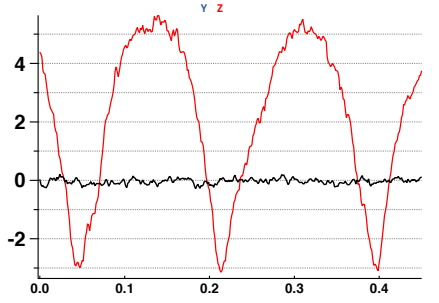

(n) z-pump-centered 13.5 FSR Local Oscillator Phase (arb. unit)

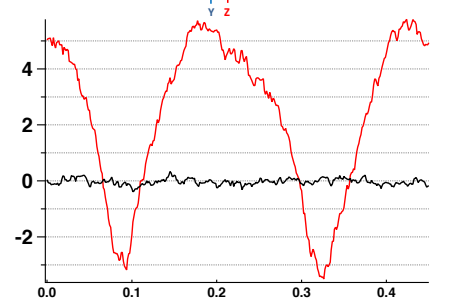

(o) z-pump-centered 14.5 FSR

Local Oscillator Phase (arb. unit)

Figure 5.32: One-wire case z pump centered nullifiers squeezing measurements. 


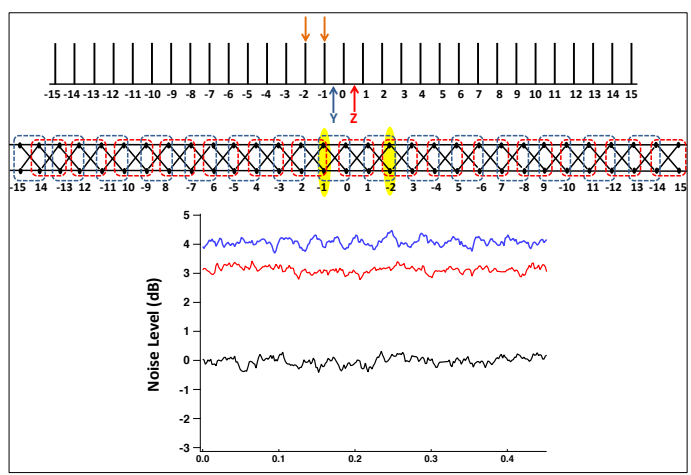

(a) One-wire Modes (-1) and (-2)

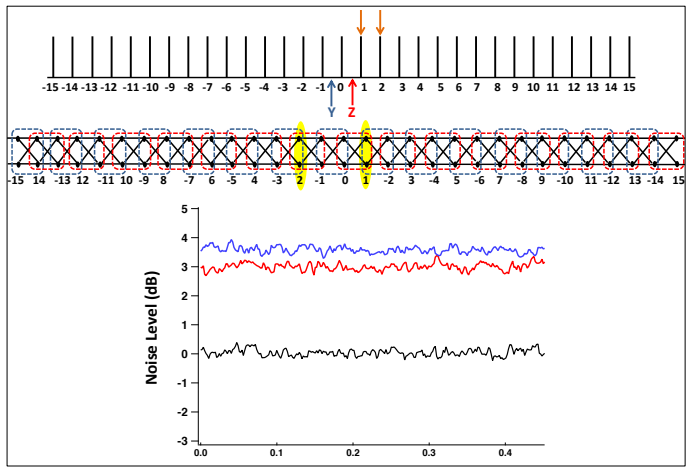

(c) One-wire Modes 1 and 2

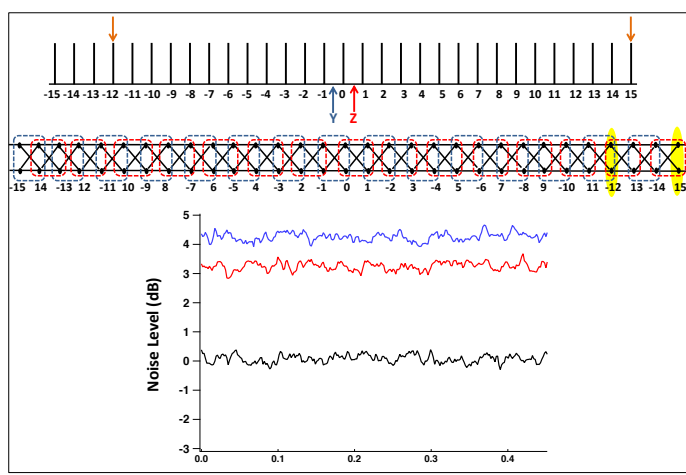

(b) One-wire Modes (-12) and 15

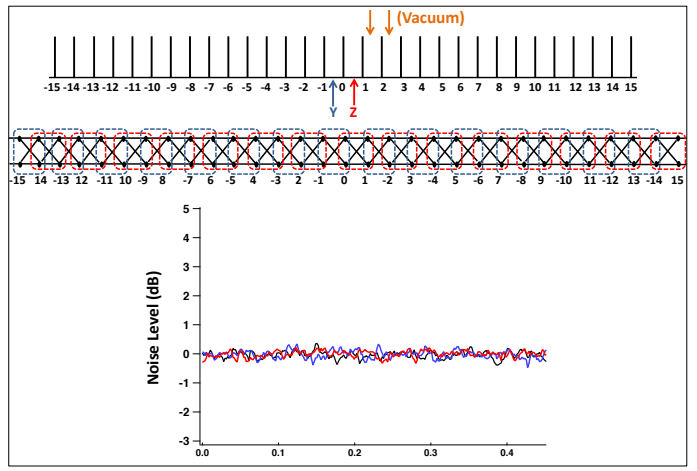

(d) One-wire Modes vacuum

Figure 5.33: Wrong-frequency measurements for one-wire case. Antisqueezing was observed between non-neighboring modes in one wire (Fig 5.33(a), 5.33(b), 5.33(c)). Shot noise level was measured when the local oscillator frequencies were tuned to that between modes as in Fig. 5.33(d). Note that in Fig. 5.33(c), Modes 1 and 2 would have been squeezed in the two-wire case but have no connection here, showing the one-wire and two-wires cases are indeed different from each other. The yellow ellipses indicate the modes we are measuring for each case. Black traces are the shot noise level, blue traces are the y pump centered nullifiers and red traces are the $\mathrm{z}$ pump centered nullifiers. In the figure, the horizontal axis is the scan of the local oscillator's phase in arbitrary units. 


\subsubsection{Two-wire case}

We tuned the two pumps further so $m=2$ for $\left|p_{y}-p_{z}\right|=2 m$ for two wire case.

We measured the nullifiers whose modes are symmetric about the y- or z- pump respectively and obtained squeezing for all of them. First I will showcase an example of two of them to see the qualitative properties.

As predicted above, changing the pump splitting to $m=2$ should yield two identical wires. Figure 5.34 show measurements demonstrating the unit cell of one of the wires. Note, in particular, that the successful nullifier measurement of the left figure of Figure 5.34 is the same as that of Figure 5.30, which wasn't a nullifier for the $m=1$ pump splitting. Another such "devil's advocate" check is displayed in Figure 5.35, in which cross correlations between the two wires are shown to be absent, even though this very same measurement yielded squeezing for $m=1$. We confirmed that 2 identical copies of a 30-mode dual-rail cluster state were generated in the QOFC.
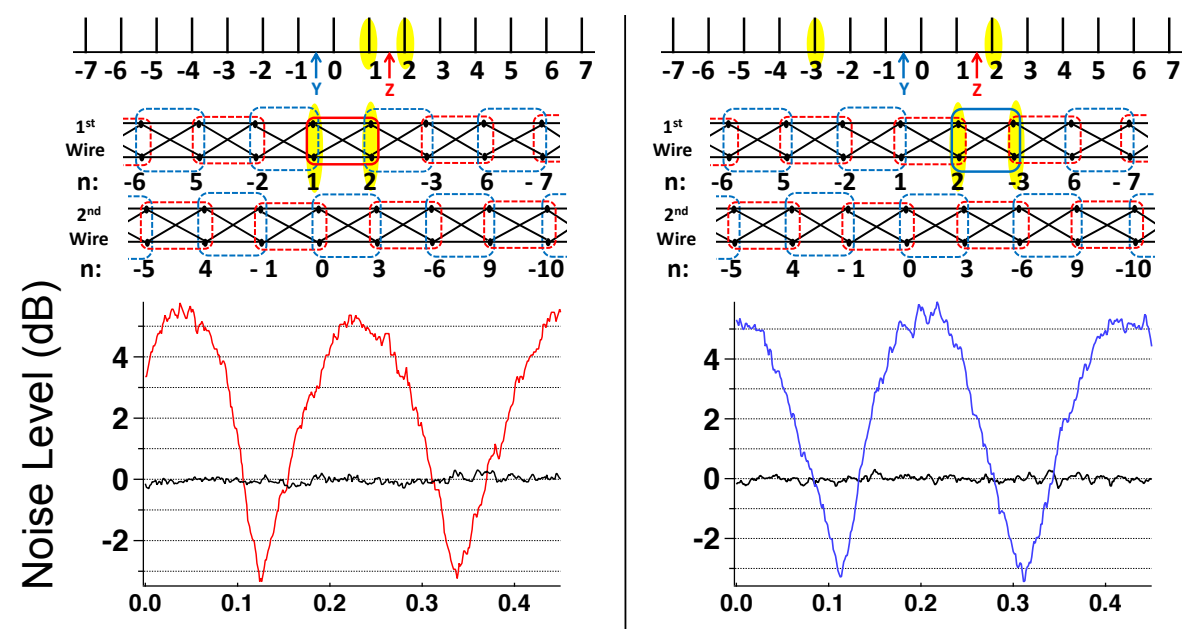

Figure 5.34: Zero-span spectrum analyzer traces of raw squeezing measurements for $m=2$ quantum wire. Figure legends same as Figure 5.29. 


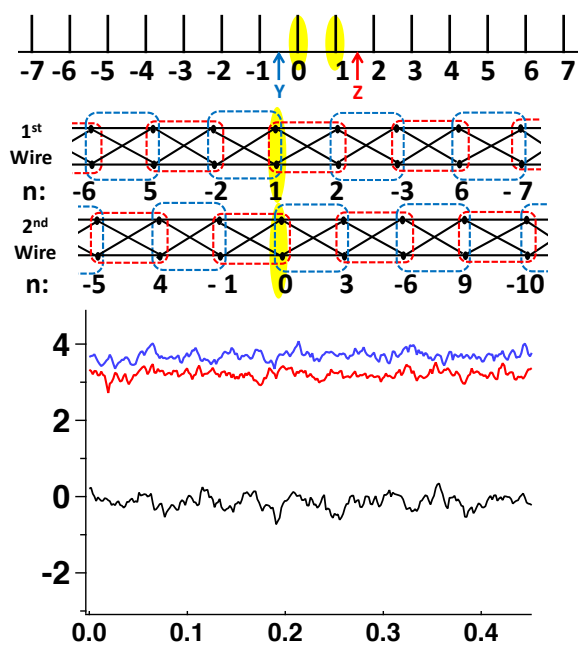

Figure 5.35: "Wrong" modes checks for two-wire case. $n=1$ and $n=0$ modes are not connected in the wire gragh state, so only excess quantum noise was detected, no squeezing observed, as expected. Figure legends same as in Figure 5.29.

The squeezing levels are constant throughout all the nullifiers indicating no sign of entanglement loss as we move further from the center. Again, the number of modes we measured for each wire in the two-wire case was only limited due to the EOM measurement ability, not the state itself. A complete squeezing measurements for two-wire case are shown in Figure 5.36 and 5.37.

Similar to the one-wire case, the wrong-frequency checks for the two-wire case only have antisqueezing or shot noise throughout the checks and no squeezing was detected at any time. The wrong-frequency checks show that there is no connection between the two wires and thus they are two independent wires. (Strictly, in order to show the independency of each wire, measurements between all the modes between the two wires are needed, but given the extremely large number of modes in a wire such measurements are tedious and beyond the EOM measure limit so we measured 


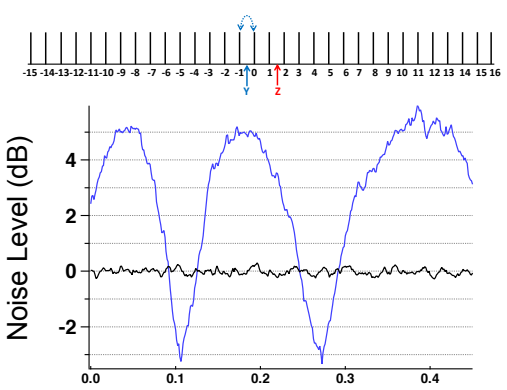

(a) y-pump-centered 0.5 FSR
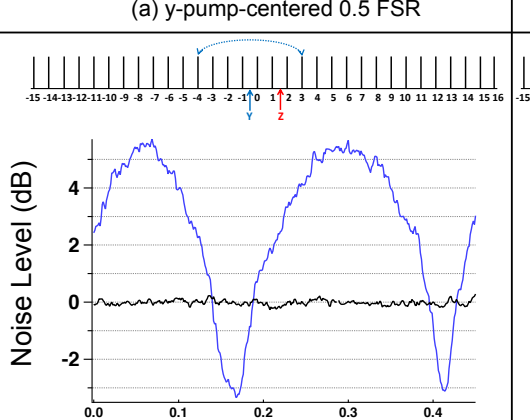

(d) y-pump-centered 3.5 FSR
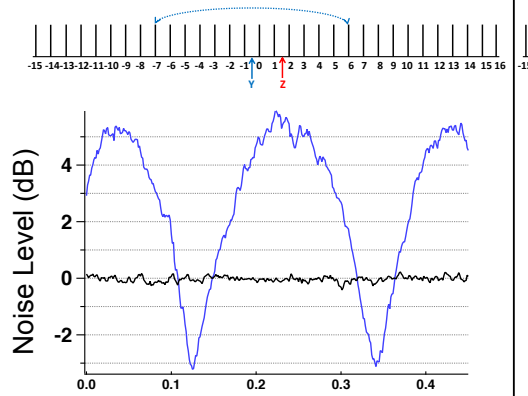

(g) y-pump-centered 6.5 FSR
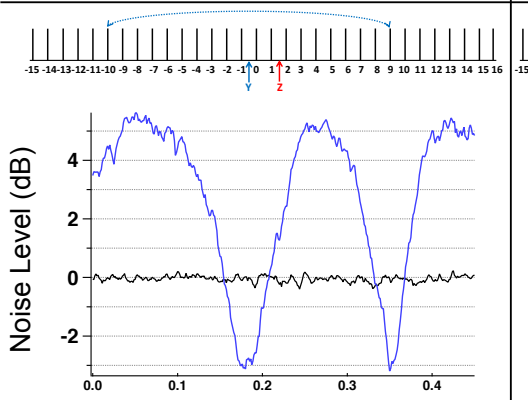

(j) y-pump-centered 9.5 FSR
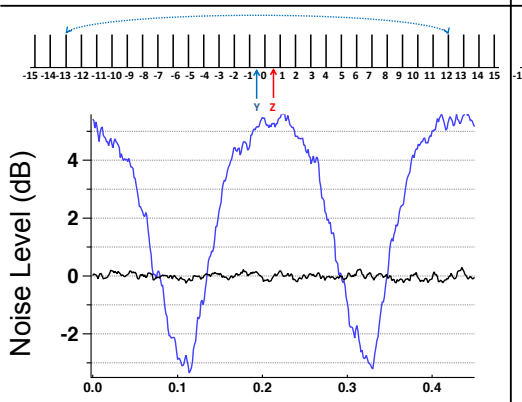

(m) y-pump-centered 12.5 FSR

Local Oscillator Phase (arb. unit)

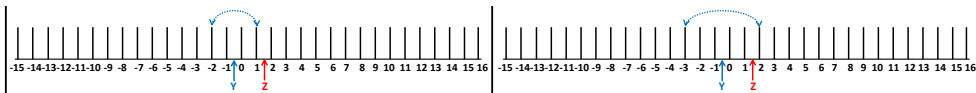

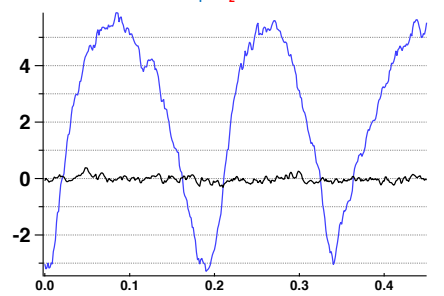

(b) y-pump-centered 1.5 FSR

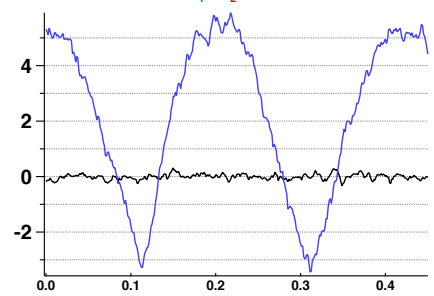

(c) y-pump-centered 2.5 FSR

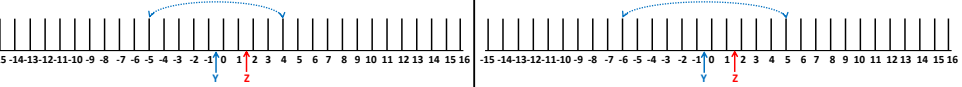

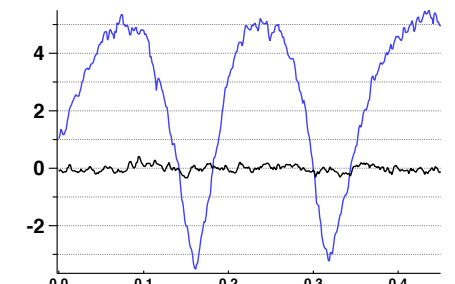

(e) y-pump-centered 4.5 FSR

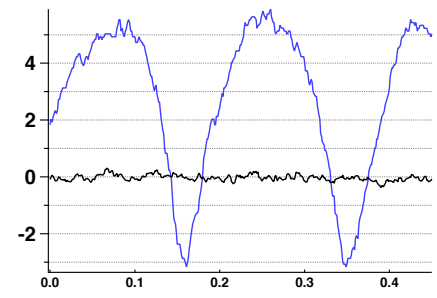

(f) y-pump-centered 5.5 FSR

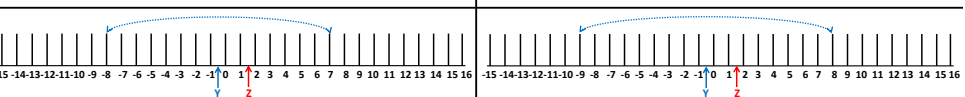

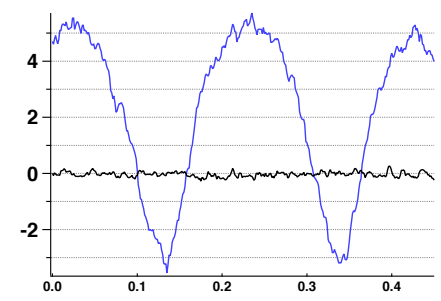

(h) y-pump-centered 7.5 FSR

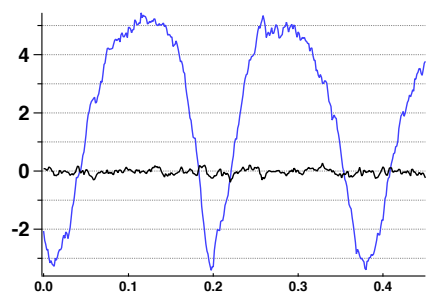

(i) y-pump-centered 8.5 FSR

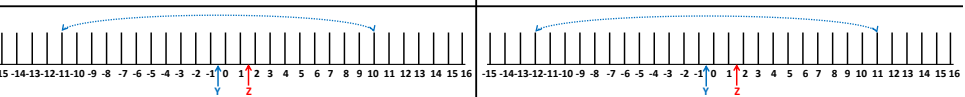

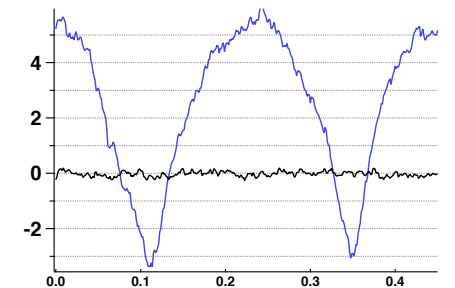

(k) y-pump-centered 10.5 FSR

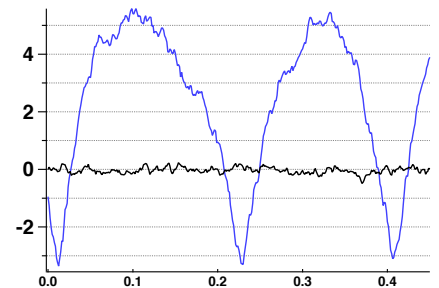

(I) y-pump-centered 11.5 FSR

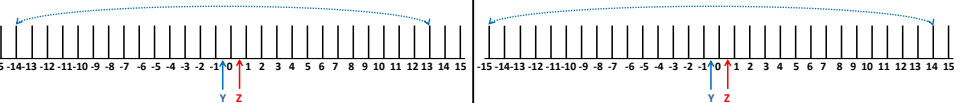

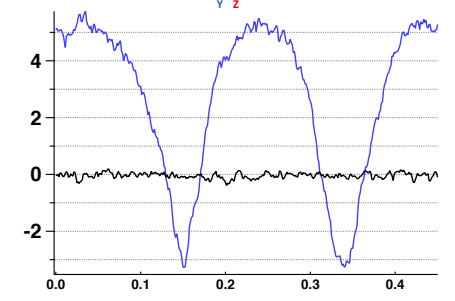

(n) y-pump-centered 13.5 FSR ocal Oscillator Phase (arb. unit)

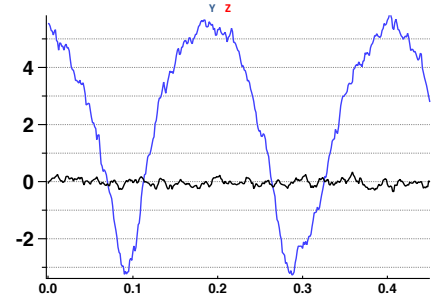

(o) y-pump-centered 14.5 FSR Local Oscillator Phase (arb. unit)

Figure 5.36: Two-wire case y pump centered nullifiers squeezing measurements. 


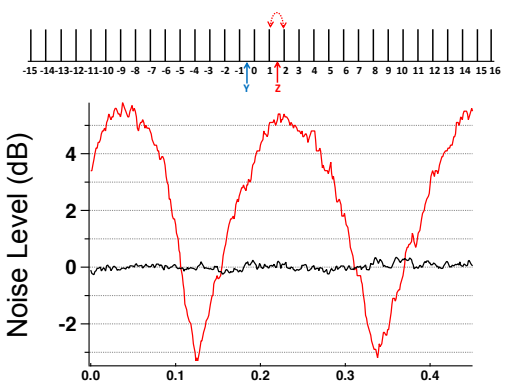

(a) z-pump-centered 0.5 FSR

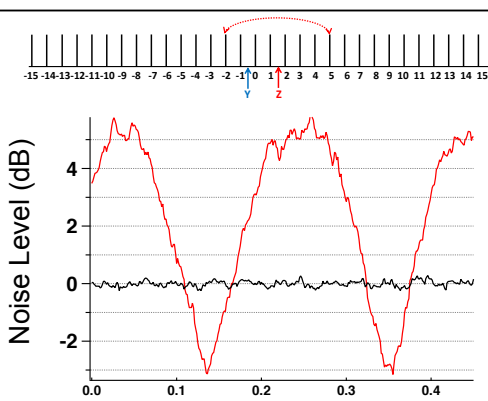

(d) z-pump-centered 3.5 FSR
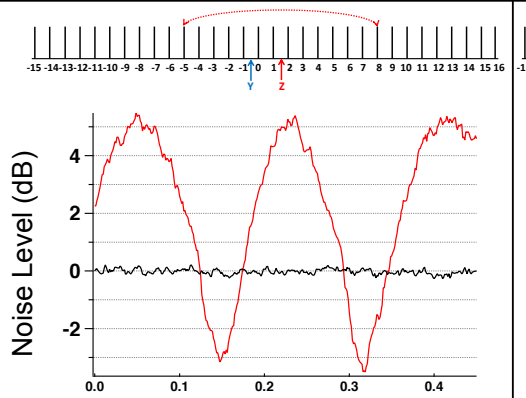

(g) z-pump-centered 6.5 FSR
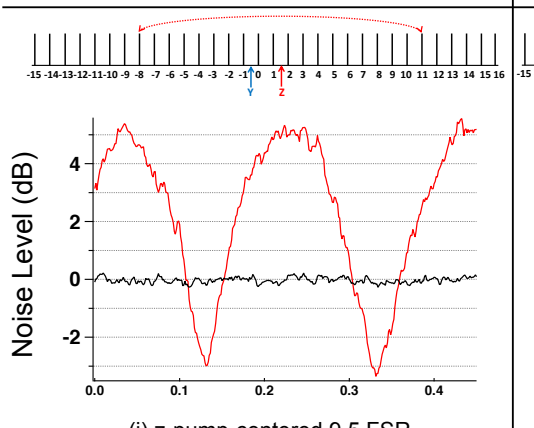

(j) z-pump-centered 9.5 FSR

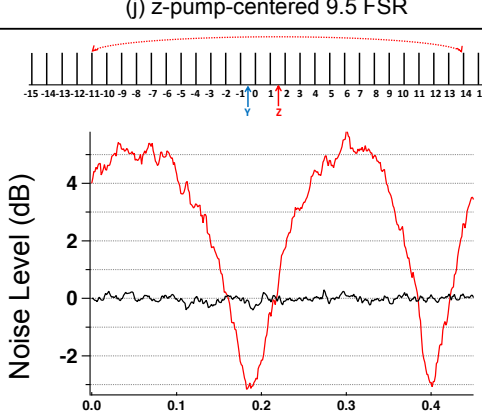

(m) z-pump-centered 12.5 FSR

Local Oscillator Phase (arb. unit)

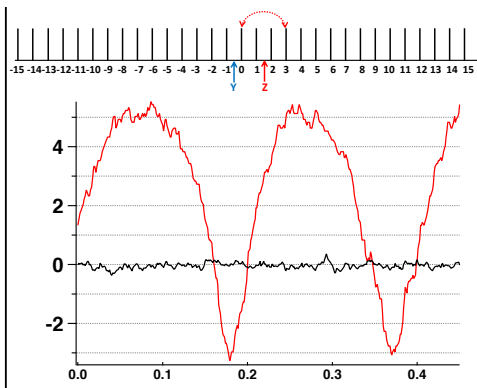

(b) z-pump-centered 1.5 FSR

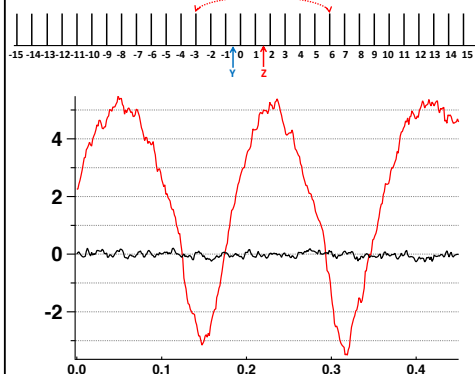

(e) z-pump-centered 4.5 FSR

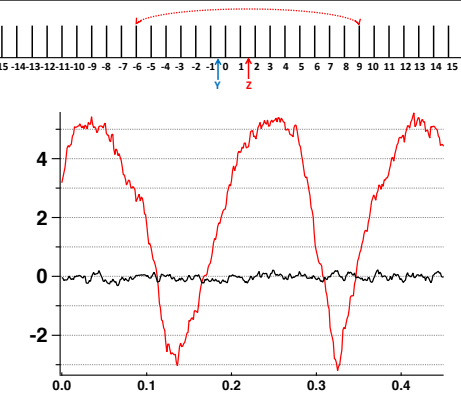

(h) z-pump-centered 7.5 FSR

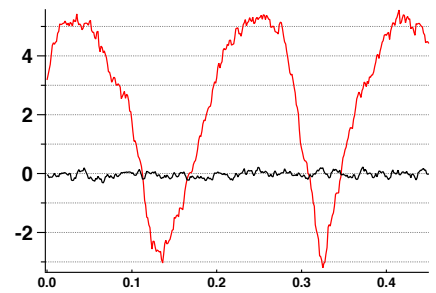

(f) z-pump-centered 5.5 FSR
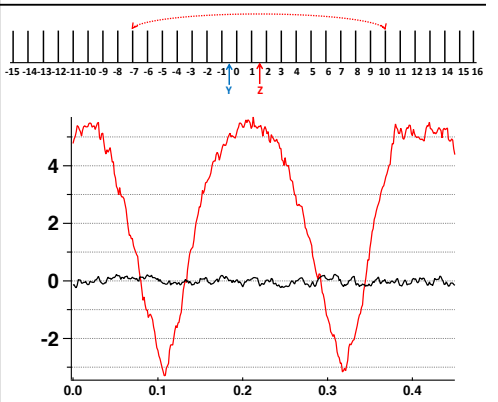

(i) z-pump-centered $8.5 \mathrm{FSR}$

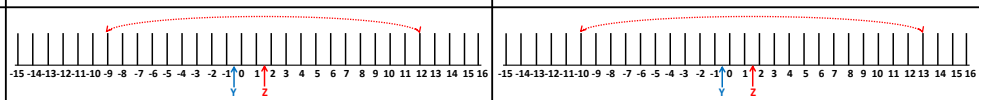

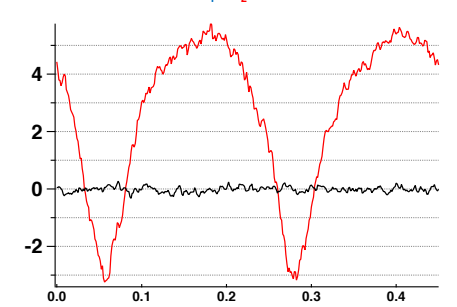

(k) z-pump-centered 10.5 FSR

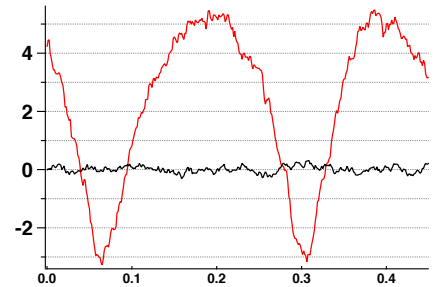

(I) z-pump-centered 11.5 FSR

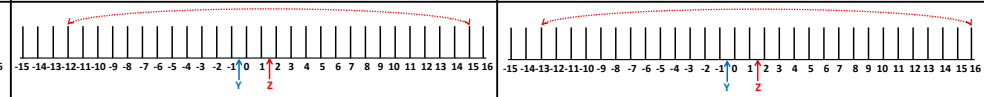

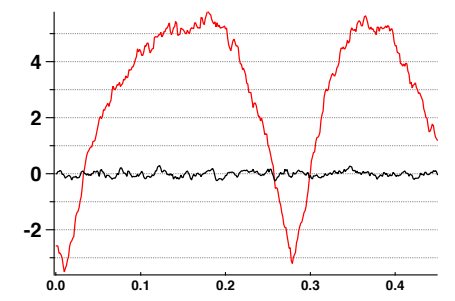

(n) z-pump-centered 13.5 FSR Local Oscillator Phase (arb. unit)

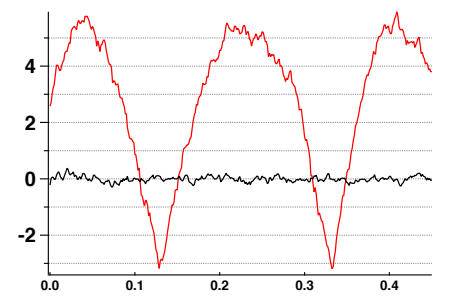

(o) z-pump-centered 14.5 FSR Local Oscillator Phase (arb. unit)

Figure 5.37: Two-wire case z pump centered nullifiers squeezing measurements. 
a few to show no sign of connection, which can be generalized.)

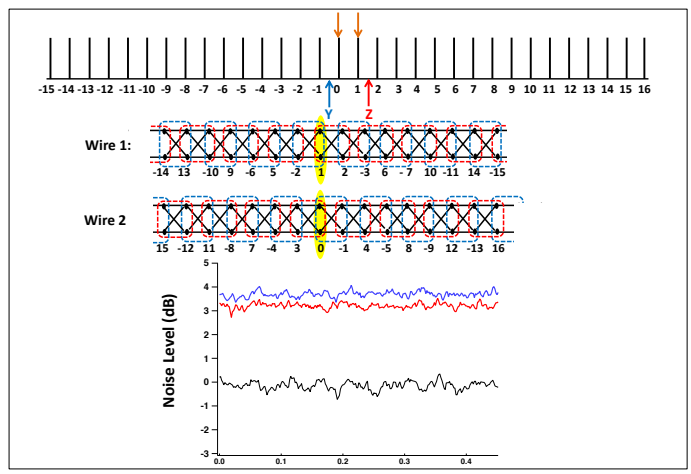

(a) Two-wire Modes 1 and 0

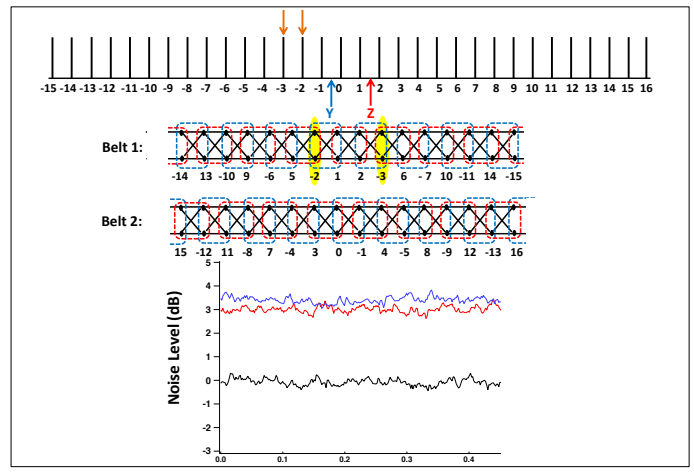

(c) Two-wire Modes (-2) and (-3)

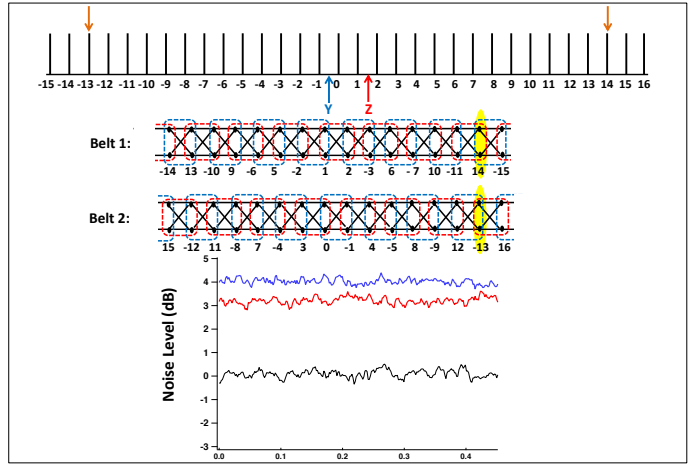

(b) Two-wire Modes 14 and (-13)

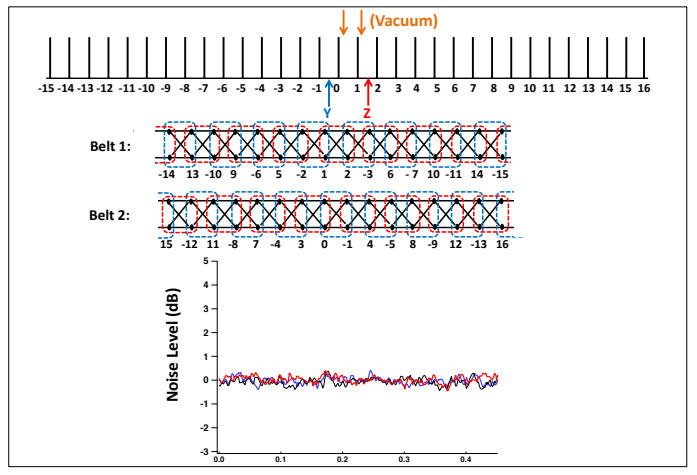

(d) Two-wire Modes vacuum

Figure 5.38: Wrong-frequency measurements for two-wire case. Antisqueezing was observed either between modes in the same wire (Fig 5.38(c) or modes in different wires (Fig 5.38(a), 5.38(b)), showing that only the neighboring modes in the same wire are connected, confirming the independent two-wire structure. Shot noise level was obtained when measuring frequencies between modes (Fig 5.38(d)). Note that in Fig 5.38(a) and 5.38(b), Modes 1 and 0 or Modes 14 and (-13) would have been squeezed in the one-wire case but have no connection here. The yellow ellipses indicate the modes we are measuring for each case. Black traces are the shot noise level, blue traces are the y pump centered nullifiers and red traces are the z pump centered nullifiers. In the figure the horizontal axis is the scan of the local oscillator's phase in arbitrary units. 


\subsubsection{Electronic noise correction}

The squeezing traces we show in the figures are the original raw measurements without any correction. The actual squeezing should be more after taking account the effects of the detector's electronic noise. Electronic noise, also known as dark noise, is about $13 \mathrm{~dB}$ below the shot noise (shown in Figure 5.39).

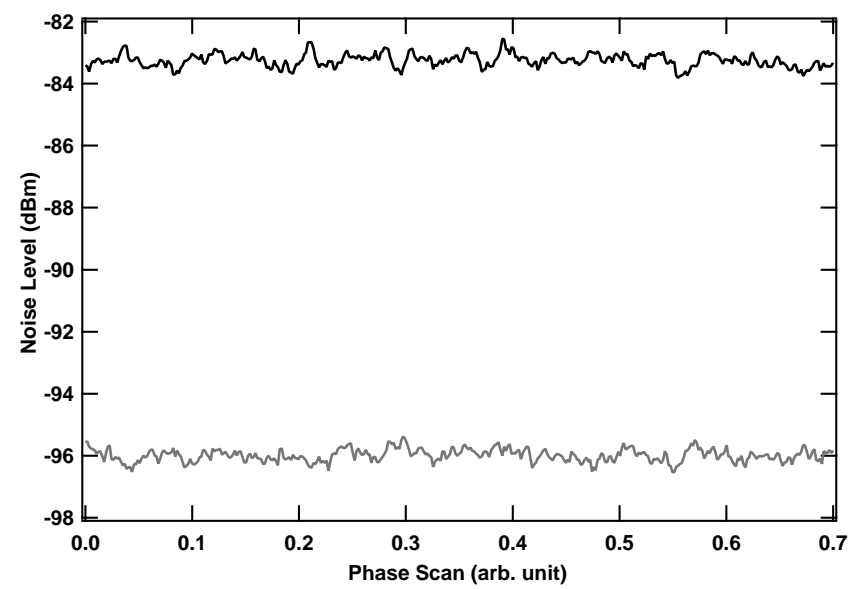

Figure 5.39: Electronic noise measurement. Black (top trace): LO shot noise; grey (bottom trace): electronic noise.

The actual squeezing level is

$$
S_{a c t}=10 \log \eta_{a c t}=10 \log \frac{V_{s q}}{V_{s n}}
$$

While the experimentally measured squeezing level, contaminated by the electronic noise, is

$$
S_{e x p}=10 \log \eta_{e x p}=10 \log \frac{V_{s q}+V_{e n}}{V_{s n}+V_{e n}}=10 \log \frac{\eta_{a c t}+\frac{V_{e n}}{V_{s n}}}{1+\frac{V_{e n}}{V_{s n}}}
$$

where $V_{s q}$ is the variance of the squeezing signal, $V_{s n}$ is the variance of the shot noise 
Table 1. Electronic Noise Correction for different Squeezing Levels

\begin{tabular}{|c|c|c|c|c|c|c|}
\hline Measured Squeezing (dB) & -3 & -3.1 & -3.2 & -3.3 & -3.4 & -3.5 \\
\hline Actual Squeezing (dB) & -3.22 & -3.33 & -3.44 & -3.55 & -3.67 & -3.78 \\
\hline Squeezing Increase (dB) & 0.22 & 0.23 & 0.24 & 0.25 & 0.27 & 0.28 \\
\hline
\end{tabular}

and $V_{e n}$ is the variance of the electronic noise. So we have

$$
\eta_{a c t}=\left(\eta_{\text {exp }}-1\right) \frac{V_{e n}}{V_{s n}}+\eta_{\text {exp }}
$$

Given our experiment's squeezing level, after the correction, the squeezing level increases $>0.2 \mathrm{~dB}$, as shown in Table 1 . 


\subsection{The van Loock-Furusawa inseparability crite- ria}

This entanglement criterion is the generalization to the multipartite case of the Duan-

Simon criterion $[71,72]$, which is itself the CV version of the Peres-Horodecki criterion $[73,74]$.

We use the van Loock-Furusawa (vLF) separability inequalities [37,63]. We consider all possible separable bipartitions in our set of entangled modes and enumerate the necessary conditions for the separability. If the inequalities for the necessary conditions of separability for all the cases are violated, we obtain the sufficient conditions for the full inseparability.

A key point here is that we are dealing with cluster states, in which quantum correlations only involve the nearest neighbors. Thus, we only need to examine the separability of the latter and may use the graph nullifiers as the test observables in building the vLF inequalities.

As was already detailed in the supplemental material of Ref. [35] (see also Refs. $[75,76])$, the nullifiers

$$
\begin{aligned}
& Q_{p_{z}-n, n}\left(r_{z}\right)=\left\{\left[Q_{n}^{(z)}+Q_{n}^{(y)}\right]-\left[Q_{p_{z}-n}^{(z)}+Q_{p_{z}-n}^{(y)}\right]\right\} e^{-r_{z}} \\
& P_{p_{z}-n, n}\left(r_{z}\right)=\left\{\left[P_{n}^{(z)}+P_{n}^{(y)}\right]+\left[P_{p_{z}-n}^{(z)}+P_{p_{z}-n}^{(y)}\right]\right\} e^{-r_{z}} \\
& Q_{p_{y}-n, n}\left(r_{y}\right)=\left\{\left[Q_{p_{y}-n}^{(z)}-Q_{p_{y}-n}^{(y)}\right]-\left[Q_{n}^{(z)}-Q_{n}^{(y)}\right]\right\} e^{-r_{y}} \\
& P_{p_{y}-n, n}\left(r_{y}\right)=\left\{\left[P_{p_{y}-n}^{(z)}-P_{p_{y}-n}^{(y)}\right]+\left[P_{n}^{(z)}-P_{n}^{(y)}\right]\right\} e^{-r_{y}} .
\end{aligned}
$$


can be written in a more compact way using the generalized quadratures $A(\theta)=$ $\left(a e^{-i \theta}+e^{i \theta}\right) / \sqrt{2}$ :

$$
\begin{aligned}
& A_{p_{z}-n, n}\left(\theta, r_{z}\right)=\left\{\left[A_{n}^{(z)}(\theta)+A_{n}^{(y)}(\theta)\right]-\left[A_{p_{z}-n}^{(z)}(-\theta)+A_{p_{z}-n}^{(y)}(-\theta)\right]\right\} e^{-r_{z}} \\
& A_{p_{y}-n, n}\left(\theta, r_{y}\right)=\left\{\left[A_{n}^{(z)}(\theta)-A_{n}^{(y)}(\theta)\right]-\left[A_{p_{y}-n}^{(z)}(-\theta)-A_{p_{y}-n}^{(y)}(-\theta)\right]\right\} e^{-r_{y}} .
\end{aligned}
$$

One can see that $\theta=0$ yields Eqs. (5.66) and (5.68) whereas $\theta=\pi / 2$ yields Eqs. (5.67) and (5.69). It is worth noting that the squeezing is independent of $\theta[75,76]$, hence any value of $\theta$ will do. (However, it is still important to measure at both angles in quadrature, say for the EPR paradox or entanglement in general, since the singlemode $\left.\left[A(\theta), A\left(\theta \pm \frac{\pi}{2}\right)\right] \neq 0.\right)$

Look at the Y-pump-centered nullifiers first:

$$
A_{-}(\theta)=\left[A(\theta)_{n_{3} z}-A(-\theta)_{n_{4} z}\right]-\left[A(\theta)_{n_{3} y}-A(-\theta)_{n_{4} y}\right]
$$

. Write it into the two quadrature nullifier form:

$$
\begin{gathered}
Q_{-}\left(n_{3}, n_{4}\right)=\left(Q_{n_{3} z}-Q_{n_{4} z}\right)-\left(Q_{n_{3} y}-Q_{n_{4} y}\right) \\
P_{-}\left(n_{3}, n_{4}\right)=\left(P_{n_{3} z}+P_{n_{4} z}\right)-\left(P_{n_{3} y}+P_{n_{4} y}\right)
\end{gathered}
$$

where frequency indexes $n_{3}$ and $n_{4}$ satisfy the phase matching condition for yyy crystal $n_{3}+n_{4}=p_{y}$. We checked that both $Q_{-}$and $P_{-}$have the same squeezing level by changing the phase of the EOM's driving signal, and this is because the value of phase $\theta$ in Eq. 5.72 does not change the squeezing. 
Similarly, the Z-pump-centered nullifier is:

$$
A_{+}(\theta)=\left[A(\theta)_{n_{1} z}-A(-\theta)_{n_{2} z}\right]+\left[A(\theta)_{n_{1} y}-A(-\theta)_{n_{2} y}\right]
$$

where $n_{1}+n_{2}=p_{z}$. Write in the quadrature form:

$$
\begin{gathered}
Q_{+}\left(n_{1}, n_{2}\right)=\left(Q_{n_{1} z}-Q_{n_{2} z}\right)+\left(Q_{n_{1} y}-Q_{n_{2} y}\right) \\
P_{+}\left(n_{1}, n_{2}\right)=\left(P_{n_{1} z}+P_{n_{2} z}\right)+\left(P_{n_{1} y}+P_{n_{2} y}\right)
\end{gathered}
$$

Let us look at four modes $n_{3 z}, n_{4 z}, n_{3 y}, n_{4 y}$ and their separability conditions.

\subsubsection{One mode- three mode bipartitions}

$\left(n_{3 z}\right)$ separable from $\left(n_{3 y}, n_{4 y}, n_{4 z}\right)$ If mode $n_{3 z}$ (the resonant mode with frequency index 3 and $z$ polarization) is separable from the other three modes, the variances of the nullifiers satisfy the inequality:

$$
\left(\Delta Q_{-}\left(n_{3}, n_{4}\right)\right)^{2}+\left(\Delta P_{-}\left(n_{3}, n_{4}\right)\right)^{2} \geq \frac{1}{2}(|1|+|-1+1-1|)=1
$$

$\left(n_{4 z}\right)$ separable from $\left(n_{3 z}, n_{3 y}, n_{4 y}\right)$ If mode $n_{4 z}$ is separable from the other three modes, the variances of the nullifiers satisfy the inequality:

$$
\left(\Delta Q_{-}\left(n_{3}, n_{4}\right)\right)^{2}+\left(\Delta P_{-}\left(n_{3}, n_{4}\right)\right)^{2} \geq \frac{1}{2}(|-1|+|1+1-1|)=1
$$

$\left(n_{3 y}\right)$ separable from $\left(n_{3 z}, n_{4 z}, n_{4 y}\right)$ If mode $n_{3 y}$ is separable from the other three modes, the variances of the nullifiers satisfy the inequality:

$$
\left(\Delta Q_{-}\left(n_{3}, n_{4}\right)\right)^{2}+\left(\Delta P_{-}\left(n_{3}, n_{4}\right)\right)^{2} \geq \frac{1}{2}(|1|+|1-1-1|)=1
$$


$\left(n_{4 y}\right)$ separable from $\left(n_{3 z}, n_{4 z}, n_{3 y}\right)$ If mode $n_{4 y}$ is separable from the other three modes, the variances of the nullifiers satisfy the inequality:

$$
\left(\Delta Q_{-}\left(n_{3}, n_{4}\right)\right)^{2}+\left(\Delta P_{-}\left(n_{3}, n_{4}\right)\right)^{2} \geq \frac{1}{2}(|-1|+|1-1+1|)=1
$$

\subsubsection{Two-mode bipartitions}

$\left(n_{3 z}, n_{3 y}\right)$ separable from $\left(n_{4 z}, n_{4 y}\right)$ If modes $n_{3 z}$ and $n_{3 y}$ are separable from modes $n_{4 z}$ and $n_{4 y}$, the variances of the nullifiers satisfy the inequality:

$$
\left(\Delta Q_{-}\left(n_{3}, n_{4}\right)\right)^{2}+\left(\Delta P_{-}\left(n_{3}, n_{4}\right)\right)^{2} \geq \frac{1}{2}(|1+1|+|-1-1|)=2
$$

$\left(n_{3 z}, n_{4 z}\right)$ separable from $\left(n_{3 y}, n_{4 y}\right)$ If modes $n_{3 z}$ and $n_{4 z}$ are separable from modes $n_{3 y}$ and $n_{4 y}$, the variances of the nullifiers satisfy the inequality:

$$
\left(\Delta Q_{-}\left(n_{3}, n_{4}\right)\right)^{2}+\left(\Delta P_{+}\left(n_{3}, n_{5}\right)\right)^{2} \geq \frac{1}{2}(|1+0|+|-1+0|)=1
$$

where $n_{3}+n_{5}=n_{\text {zpump }}$ and $P_{+}\left(n_{3}, n_{5}\right)$ is a $z$ pump centered nullifier.

$\left(n_{3 z}, n_{4 y}\right)$ separable from $\left(n_{4 z}, n_{3 y}\right)$ If modes $n_{3 z}$ and $n_{4 y}$ are separable from modes $n_{4 z}$ and $n_{3 y}$, the variances of the nullifiers satisfy the inequality:

$$
\left(\Delta Q_{-}\left(n_{3}, n_{4}\right)\right)^{2}+\left(\Delta P_{+}\left(n_{3}, n_{5}\right)\right)^{2} \geq \frac{1}{2}(|1+0|+|0-1|)=1
$$

\subsubsection{Sufficient conditions for inseparability}

The inequalities for each case above are necessary conditions for separability, and a violation of them leads to the sufficient conditions for inseparability. A sufficient 
condition for the inseparability for all the cases is that the sum of the $P$ and $Q$ nullifiers' variances be smaller than one: $\left(\Delta Q_{-}\left(n_{3}, n_{4}\right)\right)^{2}+\left(\Delta P_{-}\left(n_{3}, n_{4}\right)\right)^{2}<1$ and $\left(\Delta Q_{-}\left(n_{3}, n_{4}\right)\right)^{2}+\left(\Delta P_{+}\left(n_{3}, n_{5}\right)\right)^{2}<1$. When these sufficient conditions are satisfied, the four modes $n_{3 z}, n_{4 z}, n_{3 y}$ and $n_{4 y}$ are not separable into any subsystems and thus they are entangled. Similar results apply to the Z-pump-centered four modes $n_{1 z}$, $n_{2 z}, n_{1 y}$ and $n_{2 y}$. And once every four modes are inseparable and their overlapping neighboring four modes are inseparable as well, the whole wire's modes are inseparable because of the transitive property of each 4-mode unit's inseparability. A stronger but simpler sufficient condition for the overall inseparability can be chosen as

$$
\begin{aligned}
\left(\Delta A_{+}(\theta)\right)^{2} & <\frac{1}{2} \\
\left(\Delta A_{-}(\theta)\right)^{2} & <\frac{1}{2}
\end{aligned}
$$

This corresponds to the $-3 \mathrm{~dB}$ squeezing level for $A_{+}$and $A_{-}$, and when the squeezing level is more than this threshold all the modes are inseparable, as we've shown experimentally. 


\section{Chapter 6}

\section{Conclusion}

This thesis has introduced the one-way quantum computer and the related optical and quantum optical background to implement the building of the cluster states that are useful for one-way quantum computing and other quantum tasks.

The cluster state we have obtained experimentally is a 60 -mode dual-rail structured quantum wire, with each mode unambiguously defined with its frequency and optical polarization. Furthermore, by simply adjusting only one experimental parameter, the two pumps frequency spacing, multiple copies of the same structured wires can be readily obtained. We have verified experimentally that two 30-mode dual-rail structured quantum wires were obtained simply by increasing the two pumps frequency spacing while keeping other experimental parameters unchanged. This easy and convenient generation makes our method very efficient in producing multiple copies simultaneously and enables potential quantum communication and teleportation among them.

We have shown that a square grid cluster state and a higher dimensional cluster 
state (3-dimensinal lattice and even a 4-dimensional hypercubic structure) can be built using these quantum wires as building blocks [64]. The number of OPO needed increases linearly as the number of dimensions increases (2 OPOs needed for a 2dimensional cluster state, 3 OPOs needed for a 3-dimensional cluster state, etc.), which is feasible and attainable experimentally. It shows great experimental simplicity compared with the previous proposal for square grid cluster states [31].

Very recently, we have found another method to generate the square grid cluster state by again using the dual-rail wires as the building blocks cite new paper. However, this method is novel and new because it will combine the time and frequency domains and thus the cluster state obtained will be a hybrid system. It uses the time delay as well as the frequency domain, and as a result, only a single OPO is needed for the generation of a large square-grid cluster state. More details can be found in (cite new paper).

Overall, this thesis has discussed the experimental generation and characterization of one 60-mode copy and two 30-mode copies of a dual-rail quantum-wire cluster state in the quantum optical frequency comb of a bimodally pumped OPO. It is the largest entangled state ever created whose subsystems are all available simultaneously. Moreover, the entanglement is not of an arbitrary type, but a carefully engineered, sophisticated resource a continuous variable dual-rail quantum wire [77] that has direct application in quantum computing $[56,64]$ and in experimental studies of topological order in quantum many body systems [78]. Beyond these applications, it also 
forms a basic building block for a rich, regular-lattice structure [64], some of which could not otherwise be embedded in three-dimensional space. It can also be used as a building block for the generation of time-and-frequency hybrid cluster state systems (cite new paper). The intrinsic scalability of the experimental design paves the way for a new program of experimental research into the properties and applications of these richly entangled quantum systems. 


\section{Appendix A}

\section{Appendix}

\section{A.1 Pump spectra manipulation}

It has been show that [31] we can generate square-grid cluster states by using a certain 15-frequency pump spectrum on a nonlinear crystal inside an OPO to entangle the resonant modes we desire. One of many important properties of pumping spectra is that it is nonsymmetric with respect to the carrier. As shown in Figure A.1, where $s=N-1$ and $t=N^{2}-2 N-3$, in the unit of FSR, where $N$ is the number of modes on one side in the final square grid. Each line denotes a frequency and the two different colors (red and blue) denote two orthogonal polarizing directions.

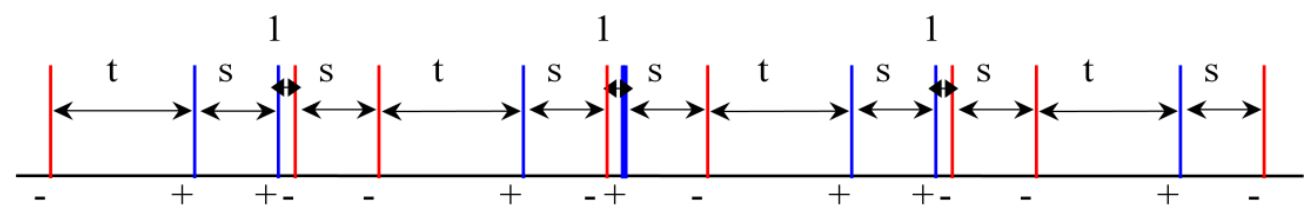

Figure A.1: The 15-frequency pump spectrum.

The 15-frequency pump spectrum is used for generating a square-grid cluster state, and to generate a crown-like cluster state (which can be "cut" into a long wire) the 
pump spectrum is different and not as complcated [31]. In the following I will talk about a general way to manipulate the pump frequency so that we can generate any pump spectra we desire. It will make use of the Single Side-band suppressed-carrier (SSB-SC) theories.

\section{A.1.1 Single-sideband Modulation}

To see how it works, let us first start with a simple pump spectrum as shown in Figure A.2. The parameter $N$ is scalable and expected to be $N \geq 5$. This pump spectrum has 3 components of specified polarizations, whose spacing is determined by $N$. The two different colors (red and blue) denote two orthogonal polarizing directions.

Now, set $N=8$ so the ratio of the spacing is $2:(N-2)=1: 3$. We approach this problem as follows: first realize the spectrum of the red lines, and then combine them with the blue line together using polarizing beam splitter.

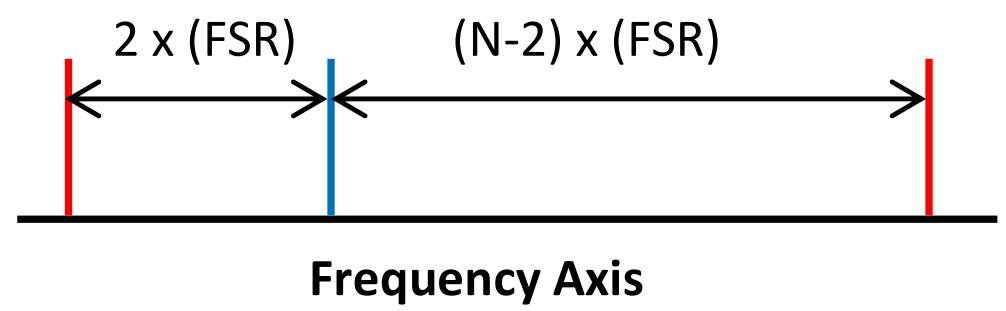

Figure A.2: The 3-frequency pump spectrum.

Use this setup:

Figure A.3 shows the schematic diagram of the setup. With this setup and adjustment of different signals, we can get the spectrum we desire. In order to get the 


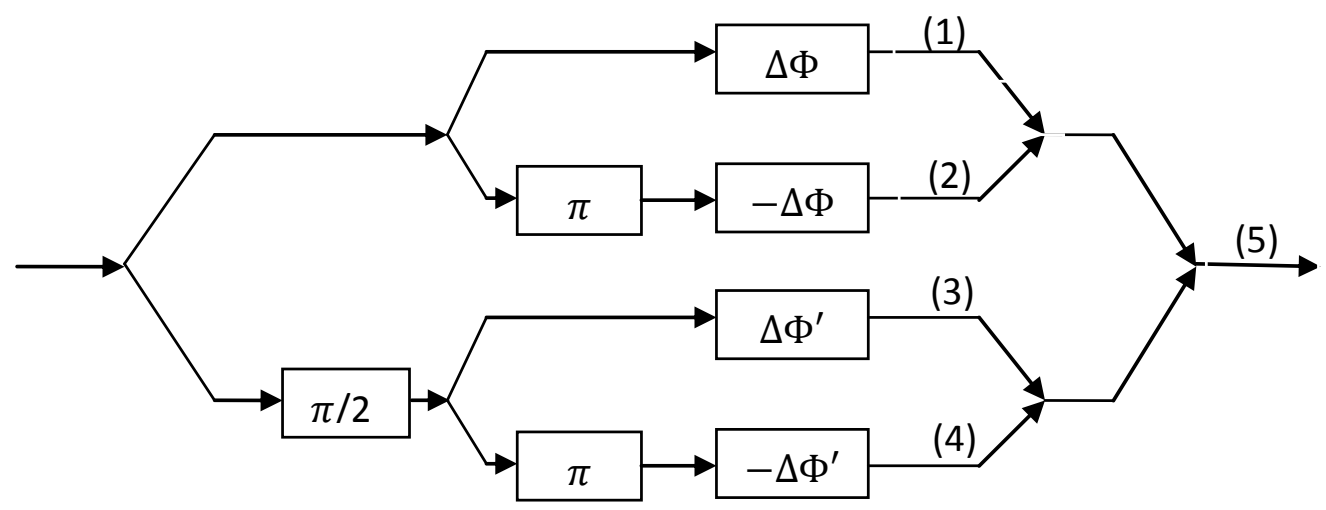

Figure A.3: The setup to generate single sideband.

spectrum of Figure A.2 we will first need to obtain the red line spectrum and blue line spectrum separately and then combine them.

Set the modulation signal $\Delta \Phi=m \cos (\Omega t), \Delta \Phi^{\prime}=-m \sin (\Omega t)$. The resulting signals at each corresponding points in Figre A.3 are:

$$
\begin{aligned}
& (1) e^{i \omega t} e^{i m \cos (\Omega t)}=e^{i \omega t} \sum_{n=-\infty}^{+\infty} i^{n} J_{n}(m) e^{i n \Omega t} \\
& (2) e^{i(\omega t+\pi)} e^{-i m \cos (\Omega t)}=e^{i \omega t}(-1) e^{i m \cos (\Omega t+\pi)}=e^{i \omega t}(-1) \sum_{n=-\infty}^{+\infty}(-i)^{n} J_{n}(m) e^{i n \Omega t} \\
& (3) e^{i\left(\omega t+\frac{\pi}{2}\right)} e^{-i m \sin (\Omega t)}=e^{i \omega t} i \sum_{n=-\infty}^{+\infty}(-1)^{n} J_{n}(m) e^{i n \Omega t} \\
& (4) e^{i\left(\omega t+\frac{\pi}{2}+\pi\right)} e^{i m \sin (\Omega t)}=e^{i \omega t}(-i) \sum_{n=-\infty}^{+\infty} i^{n} J_{n}(m) e^{i n \Omega t}
\end{aligned}
$$

The corresponding optical frequency spectra are (dashed lines denote the imaginary part, solid lines real part):

The amplitude $J_{n}(m)$, is Bessel function, with the property $J_{-n}(m)=(-1)^{n} J_{n}(m)$. The graph for the first few orders of Bessel functions is as follows: 
(1)

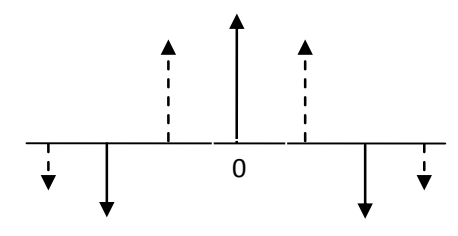

(2)

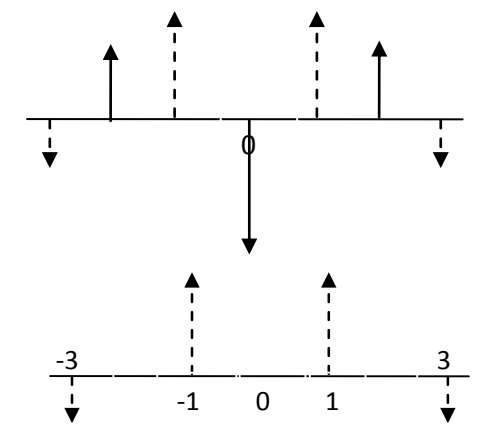

(1) $+(2)$

$(3)+(4)$

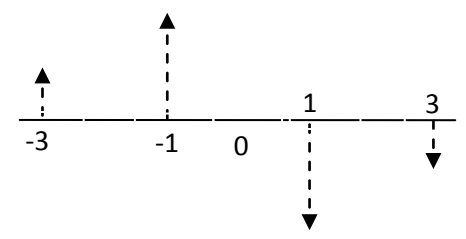

(5)

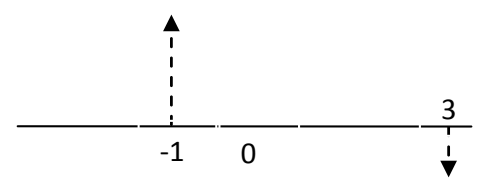

Figure A.4: The frequency spectra of corresponding points in Figure A.3. The axis unit is $2 \mathrm{xFSR}$.

This setup can be used as SSB-SC modulator in the range $m<1$ rad where $J_{3}(m) \approx 0, J_{1}(m) \gg J_{3}(m)$. However, at $m=1.8 \mathrm{rad}$, maximum fundamental output can be obtained. Thus, for the signal at point (5), only the -1 order will be shown, and thus the left most red line in Figure A.2 is obtained, with the setting 


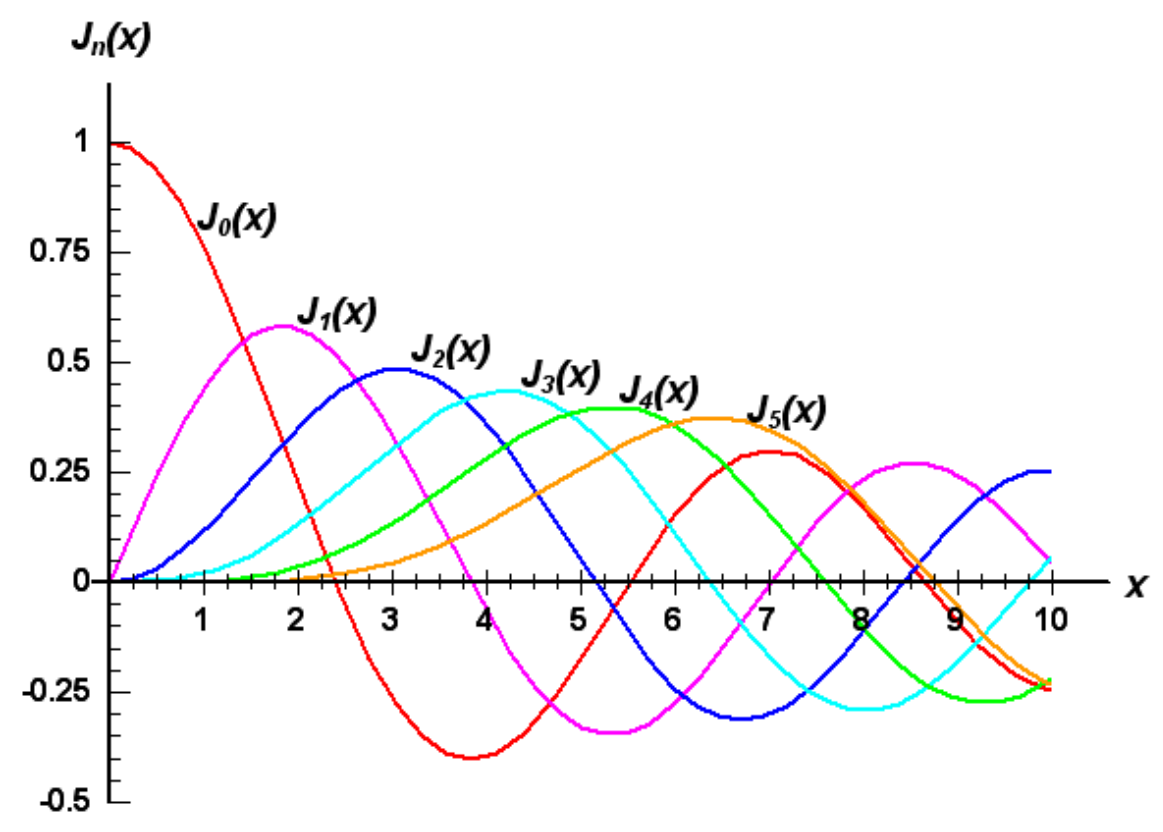

Figure A.5: Bessel functions of different orders.

$\Omega=2 \times(F S R)$.

Similarly, use the same setup, we can also get SSB on the right (positive) side. To do this, we just need to change the input modulation signal to $\Delta \Phi=m \cos \left(\Omega^{\prime} t\right), \Delta \Phi^{\prime}=$ $m \sin \left(\Omega^{\prime} t\right)$. This set of signal leads to:

$$
\begin{aligned}
& (1) e^{i \omega t} e^{i m \cos (\Omega t)}=e^{i \omega t} \sum_{n=-\infty}^{+\infty} i^{n} J_{n}(m) e^{i n \Omega t} \\
& (2) e^{i(\omega t+\pi)} e^{-i m \cos (\Omega t)}=e^{i \omega t}(-1) e^{i m \cos (\Omega t+\pi)}=e^{i \omega t}(-1) \sum_{n=-\infty}^{+\infty}(-i)^{n} J_{n}(m) e^{i n \Omega t} \\
& (3) e^{i\left(\omega t+\frac{\pi}{2}\right)} e^{-i m \sin (\Omega t)}=e^{i \omega t} i \sum_{n=-\infty}^{+\infty}(-1)^{n} J_{n}(m) e^{i n \Omega t} \\
& (4) e^{i\left(\omega t+\frac{\pi}{2}+\pi\right)} e^{i m \sin (\Omega t)}=e^{i \omega t}(-i) \sum_{n=-\infty}^{+\infty} i^{n} J_{n}(m) e^{i n \Omega t}
\end{aligned}
$$




\section{Bibliography}

[1] Richard P. Feynman. Simulating physics with computers. Int. J. Theor. Phys., 21:467-488, 1982.

[2] D. Deutsch. Quamtum theory,the church-turing principle and the universal quantum computer. Proc. R. Soc. Loud. A, 400:97, 1985.

[3] D. Deutsch and R. Jozsa. Rapid solution of problems by quantum computation. Proc. Roy. Soc. Lond. A, 439(1907):553-558, 1992.

[4] P. W. Shor. Algorithms for quantum computation: discrete logarithms and factoring. In S. Goldwasser, editor, Proceedings, $35^{\text {th }}$ Annual Symposium on Foundations of Computer Science, pages 124-134, Santa Fe, NM, 1994. IEEE Press, Los Alamitos, CA.

[5] W. H. Brattain. entry of 15 December 1947 Bell laboratary notebook, case 38139$7,1947$.

[6] Gordon Moore. Electronics Magazine, page 4, 1965. 
[7] Alan Cobham. The intrisic computational difficulty of functions. Proc. Logic, Methodology, and Philosophy of Science II, 1965.

[8] Jack Edmonds. Path, trees and flowers. Canad. J. Math, 17:449-467, 1965.

[9] Michael A. Nielsen and Isaac L. Chuang. Quantum computation and quantum information. Cambridge University Press, Cambridge, U.K., 2000.

[10] S. L. Braunstein and H. K. Lo, editors. Scalable quantum computers. Paving the way to realization. Wiley, 2001.

[11] Nicolas C. Menicucci. Fault-tolerant measurement-based quantum computing with continuous-variable cluster states. arXiv:1310.7596 [quant-ph], 2013.

[12] Daniel Gottesman, Alexei Kitaev, and John Preskill. Encoding a qubit in an oscillator. Phys. Rev. A, 64:012310, 2001.

[13] P Walther, K J Resch, T Rudolph, E Schenck, H Weinfurter, V Vedral, M Aspelmeyer, and A Zeilinger. Experimental one-way quantum computing. Nature, 434:169, 2005.

[14] B. P. Lanyon, T. J. Weinhold, N. K. Langford, M. Barbieri, D. F. V. James, A. Gilchrist, and A. G. White. Experimental demonstration of a compiled version of Shor's algorithm with quantum entanglement. Phys. Rev. Lett., 99(25):250505, 2007. 
[15] Chao-Yang Lu, Daniel E. Browne, Tao Yang, and Jian-Wei Pan. Demonstration of a compiled version of Shor's quantum factoring algorithm using photonic qubits. Phys. Rev. Lett., 99:250504, Dec 2007.

[16] P. Neumann, N. Mizuochi, F. Rempp, P. Hemmer, H. Watanabe, S. Yamasaki, V. Jacques, T. Gaebel, F. Jelezko, and J. Wrachtrup. Multipartite entanglement among single spins in diamond. Science, 320(5881):1326-1329, 2008.

[17] Alberto Politi, Jonathan C. F. Matthews, and Jeremy L. O'Brien. Shor's quantum factoring Shor's quantum factoring algorithm on a photonic chip. Science, 325:1221, 2009.

[18] Matthew Neeley, Radoslaw C. Bialczak, M. Lenander, E. Lucero, Matteo Mariantoni, A. D. O'Connell, D. Sank, H. Wang, M. Weides, J. Wenner, Y. Yin, T. Yamamoto, A. N. Cleland, and John M. Martinis. Generation of three-qubit entangled states using superconducting phase qubits. Nature, 467:570, 2010.

[19] Thomas Monz, Philipp Schindler, Julio T. Barreiro, Michael Chwalla, Daniel Nigg, William A. Coish, Maximilian Harlander, Wolfgang Hänsel, Markus Hennrich, and Rainer Blatt. 14-qubit entanglement: Creation and coherence. Phys. Rev. Lett., 106(13):130506, Mar 2011.

[20] B. P. Lanyon, C. Hempel, D. Nigg, M. Müller, R. Gerritsma, F. Zähringer, P. Schindler, J.T. Barreiro, M. Rambach, G. Kirchmair, M. Hennrich, P. Zoller, 
R. Blatt, and C. F. Roos. Universal digital quantum simulation with trapped ions. Science, 334:57, 2011.

[21] Stefanie Barz, Elham Kashefi, Anne Broadbent, Joseph F. Fitzsimons, Anton Zeilinger, and Philip Walther. Demonstration of blind quantum computing. Science, 335(6066):303-308, 2012.

[22] Xing-Can Yao, Tian-Xiong Wang, Wei-Bo Gao Hao-Ze Chen1, Austin G. Fowler, Robert Raussendorf, Zeng-Bing Chen, Nai-Le Liu, Chao-Yang Lu, You-Jin Deng, Yu-Ao Chen, and Jian-Wei Pan. Experimental demonstration of topological error correction. Nat. Photon., 482:489, 2012.

[23] R. Brunner, Y.-S. Shin, T. Obata, M. Pioro-Ladrière, T. Kubo, K. Yoshida, T. Taniyama, Y. Tokura, and S. Tarucha. Two-qubit gate of combined singlespin rotation and interdot spin exchange in a double quantum dot. Phys. Rev. Lett., 107:146801, Sep 2011.

[24] Xuedong Hu and S. Das Sarma. Charge-fluctuation-induced dephasing of exchange-coupled spin qubits. Phys. Rev. Lett., 96:100501, Mar 2006.

[25] J. H. Wesenberg, A. Ardavan, G. A. D. Briggs, J. J. L. Morton, R. J. Schoelkopf, D. I. Schuster, and K. Mølmer. Quantum computing with an electron spin ensemble. Phys. Rev. Lett., 103:070502, Aug 2009.

[26] Jarryd J. Pla, Fahd A. Mohiyaddin, Kuan Y. Tan, Juan P. Dehollain, Rajib Rahman, Gerhard Klimeck, David N. Jamieson, Andrew S. Dzurak, and Andrea 
Morello. Coherent control of a single ${ }^{29} \mathrm{Si}$ nuclear spin qubit. Phys. Rev. Lett., 113:246801, Dec 2014.

[27] Xing Rong, Jianpei Geng, Zixiang Wang, Qi Zhang, Chenyong Ju, Fazhan Shi, Chang-Kui Duan, and Jiangfeng Du. Implementation of dynamically corrected gates on a single electron spin in diamond. Phys. Rev. Lett., 112:050503, Feb 2014.

[28] Thi Ha Kyaw, Ying Li, and Leong-Chuan Kwek. Measurement-based quantum computation on two-body interacting qubits with adiabatic evolution. Phys. Rev. Lett., 113:180501, Oct 2014.

[29] O. Pfister. Graph states and carrier-envelope phase squeezing. ArXiv quantph/0701104, 2007.

[30] N. C. Menicucci, S. T. Flammia, H. Zaidi, and O. Pfister. Ultracompact generation of continuous-variable cluster states. Phys. Rev. A, 76:010302(R), 2007.

[31] Nicolas C. Menicucci, Steven T. Flammia, and Olivier Pfister. One-way quantum computing in the optical frequency comb. Phys. Rev. Lett., 101:130501, 2008.

[32] S. T. Flammia, N. C. Menicucci, and O. Pfister. The optical frequency comb as a one-way quantum computer. J. Phys. B,, 42:114009, 2009. 
[33] G. Patera, C. Navarrete-Benlloch, G.J. de Valcárcel, and C. Fabre. Quantum coherent control of highly multipartite continuous-variable entangled states by tailoring parametric interactions. Eur. Phys. J. D, 66:241, 2012.

[34] Moran Chen, Nicolas C. Menicucci, and Olivier Pfister. Experimental realization of multipartite entanglement of 60 modes of a quantum optical frequency comb. Phys. Rev. Lett., 112:120505, Mar 2014.

[35] Matthew Pysher, Yoshichika Miwa, Reihaneh Shahrokhshahi, Russell Bloomer, and Olivier Pfister. Parallel generation of quadripartite cluster entanglement in the optical frequency comb. Phys. Rev. Lett., 107(3):030505, Jul 2011.

[36] Ryuji Ukai, Noriaki Iwata, Yuji Shimokawa, Seiji Armstrong, Alberto Politi, Jun-ichi Yoshikawa, Peter van Loock, and Akira Furusawa. Demonstration of unconditional one-way quantum computations for continuous variables. Phys. Rev. Lett., 106:240504, Jun 2011.

[37] Shota Yokoyama, Ryuji Ukai, Seiji C. Armstrong, Chanond Sornphiphatphong, Toshiyuki Kaji, Shigenari Suzuki, Jun ichi Yoshikawa, Hidehiro Yonezawa, Nicolas C. Menicucci, and Akira Furusawa. Ultra-large-scale continuous-variable cluster states multiplexed in the time domain. Nat. Photon., 7:982, 2013.

[38] R. Raussendorf and H. J. Briegel. A one-way quantum computer. Phys. Rev. Lett., 86:5188, 2001. 
[39] Yuuki Tokunaga, Shin Kuwashiro, Takashi Yamamoto, Masato Koashi, and Nobuyuki Imoto. Generation of high-fidelity four-photon cluster state and quantum-domain demonstration of one-way quantum computing. Phys. Rev. Lett., 100:210501, May 2008.

[40] Ryuji Ukai, Shota Yokoyama, Jun-ichi Yoshikawa, Peter van Loock, and Akira Furusawa. Demonstration of a controlled-phase gate for continuous-variable oneway quantum computation. Phys. Rev. Lett., 107:250501, Dec 2011.

[41] Mitsuyoshi Yukawa, Ryuji Ukai, Peter van Loock, and Akira Furusawa. Experimental generation of four-mode continuous-variable cluster states. Phys. Rev. A, 78:012301, 2008.

[42] S. Tame, M. A. Bell, B. C. Di Franco, J. Wadsworth, W. and G. Rarity, J. Experimental realization of a one-way quantum computer algorithm solving simon's problem. Phys. Rev. Lett., 113:200501, Nov 2014.

[43] Kensuke Inaba, Yuuki Tokunaga, Kiyoshi Tamaki, Kazuhiro Igeta, and Makoto Yamashita. High-fidelity cluster state generation for ultracold atoms in an optical lattice. Phys. Rev. Lett., 112:110501, Mar 2014.

[44] Pei Wang, Wenjiang Fan, and Olivier Pfister. Quasi-phase-matched sumfrequency generation from a range of several $\mathrm{THz}$ at $1064 \mathrm{~nm}$ in periodically poled $\mathrm{KTiPO}_{4}$. in preparation, 2013. 
[45] Pei Wang, Moran Chen, Nicolas C. Menicucci, and Olivier Pfister. Weaving quantum optical frequency combs into continuous-variable hypercubic cluster states. Phys. Rev. A, 90:032325, Sep 2014.

[46] Nicolas C. Menicucci. Temporal-mode continuous-variable cluster states using linear optics. Phys. Rev. A, 83(6):062314, Jun 2011.

[47] O. Pfister. Quantum optics lecture notes. Spring 2012.

[48] Mark Fox. Quantum Optics- an introduction. Oxford University Press, 2006.

[49] C.K. Hong, Z.Y. Ou, and L. Mandel. Measurement of subpicosecond time intervals between two photons by interference. Phys. Rev. Lett., 59:2044, 1987.

[50] H. J. Briegel and R. Raussendorf. Persistent entanglement in arrays of interacting particles. Phys. Rev. Lett., 86:910, 2001.

[51] D. Gottesman. Stabilizer codes and quantum error correction. PhD thesis, California Institute of Technology, Pasadena, CA, 1997.

[52] Seth Lloyd and Samuel L. Braunstein. Quantum computation over continuous variables. Phys. Rev. Lett., 82:1784, 1999.

[53] Mile Gu, Christian Weedbrook, Nicolas C. Menicucci, Timothy C. Ralph, and Peter van Loock. Quantum computing with continuous-variable clusters. Phys. Rev. A, 79:062318, 2009. 
[54] Jing Zhang and Samuel L. Braunstein. Continuous-variable Gaussian analog of cluster states. Phys. Rev. A, 73(3):032318, 2006.

[55] Stephen D. Bartlett, Barry C. Sanders, Samuel L. Braunstein, and Kae Nemoto. Efficient classical simulation of continuous variable quantum information processes. Phys. Rev. Lett., 88:097904, 2002.

[56] N. C. Menicucci, P. van Loock, M. Gu, C. Weedbrook, T. C. Ralph, and M. A. Nielsen. Universal quantum computation with continuous-variable cluster states. Phys. Rev. Lett., 97:110501, 2006.

[57] B. E. A. Saleh and M. C. Teich. Fundamentals of Photonics. Wiley and Sons, New York, NY, 1991.

[58] Jonathan Roslund, Renné Medeiros de Araújo, Shifeng Jiang, Claude Fabre, and Nicolas Treps. Wavelength-multiplexed quantum networks with ultrafast frequency combs. Nat. Photon., 8:109, 2014.

[59] A. Einstein, B. Podolsky, and N. Rosen. Can quantum-mechanical description of physical reality be considered complete? Phys. Rev., 47:777, 1935.

[60] Nicolas C. Menicucci, Steven T. Flammia, and Peter van Loock. Graphical calculus for Gaussian pure states with applications to continuous-variable cluster states. Phys. Rev. A, 83:042335, 2011. 
[61] P. Hyllus and J. Eisert. Optimal entanglement witnesses for continuous-variable systems. New J. Phys., 8(4):51, 2006.

[62] See supplemental material in Appendix.

[63] P. van Loock and A. Furusawa. Detecting genuine multipartite continuousvariable entanglement. Phys. Rev. A, 67:052315, 2003.

[64] Pei Wang, Moran Chen, Nicolas C. Menicucci, and Olivier Pfister. Weaving quantum optical frequency combs into hypercubic cluster states. arXiv:1309.4105 [quant-ph], 2013.

[65] M. M. Fejer, G. A. Magel, D. H. Jundt, and R. L. Byer. Quasi-phase-matched second harmonic generation: tuning and tolerances. IEEE J. Quantum Electron., 28:2631, 1992.

[66] R. C. Pooser and O. Pfister. Observation of triply coincident nonlinearities in periodically poled $\mathrm{KTiOPO}_{4}$. Opt. Lett., 30:2635, 2005.

[67] M. Pysher, A. Bahabad, P. Peng, A. Arie, and O. Pfister. Quasi-phase-matched concurrent nonlinearities in periodically poled $\mathrm{KTiPO}_{4}$ for quantum computing over the optical frequency comb. Opt. Lett., 35:565, 2010.

[68] R. W. P. Drever, J. L. Hall, F. V. Kowalski, J. Hough, G. M. Ford, A. J. Munley, and H. Ward. Laser phase and frequency stabilization using an optical resonator. Appl. Phys. B, 31:97-105, 1983. 
[69] Gary C. Bjorklund. Frequency-modulation spectroscopy: a new method for measuring weak absorptions and dispersions. Opt. Lett., 5:15, 1980.

[70] K. Kato and E. Takaoka. Sellmeier and thermo-optic dispersion formulas for KTP. Appl. Opt., 41:5040, 2002.

[71] L.-M. Duan, G. Giedke, J.I. Cirac, and P. Zoller. Inseparability criterion for continuous variable systems. Phys. Rev. Lett., 84:2722, 2000.

[72] R. Simon. Peres-Horodecki separability criterion for continuous variable systems. Phys. Rev. Lett., 84:2726, 2000.

[73] Asher Peres. Separability criterion for density matrices. Phys. Rev. Lett., 77:1413-1415, Aug 1996.

[74] Michal Horodecki, Pawel Horodecki, and Ryszard Horodecki. Separability of mixed states: necessary and sufficient conditions. Phys. Lett. A, 223:1-8, 1996.

[75] Gerd Leuchs, Ruifang Dong, and Denis Sych. Triplet-like correlation symmetry of continuous variable entangled states. New J. Phys., 11:113040, 2009.

[76] R. Bloomer, M. Pysher, and O. Pfister. Nonlocal restoration of two-mode squeezing in the presence of strong optical loss. New J. Phys., 13:063014, 2011.

[77] Rafael N. Alexander, Seiji C. Armstrong, Ryuji Ukai, and Nicolas C. Menicucci. Noise analysis of single-qumode gaussian operations using continuous-variable cluster states. arXiv:1311.3538 [quant-ph], 2013. 
[78] Tommaso F. Demarie, Trond Linjordet, Nicolas C. Menicucci, and Gavin K. Brennen. Detecting topological entanglement entropy in a lattice of quantum harmonic oscillators. arXiv:1305.0409 [quant-ph], May 2013. 